\title{
Spherical near field acoustic holography with microphones on a rigid sphere Abstract of paper
}

Jacobsen, Finn; Hald, Jørgen; Fernandez Grande, Efren; Moreno, Guillermo

\section{Published in:}

Acoustical Society of America. Journal

Link to article, DOI:

$10.1121 / 1.2934035$

Publication date:

2008

Document Version

Publisher's PDF, also known as Version of record

Link back to DTU Orbit

Citation (APA):

Jacobsen, F., Hald, J., Fernandez Grande, E., \& Moreno, G. (2008). Spherical near field acoustic holography with microphones on a rigid sphere: Abstract of paper. Acoustical Society of America. Journal, 123(5), 33853385. https://doi.org/10.1121/1.2934035

\section{General rights}

Copyright and moral rights for the publications made accessible in the public portal are retained by the authors and/or other copyright owners and it is a condition of accessing publications that users recognise and abide by the legal requirements associated with these rights.

- Users may download and print one copy of any publication from the public portal for the purpose of private study or research.

- You may not further distribute the material or use it for any profit-making activity or commercial gain

- You may freely distribute the URL identifying the publication in the public portal 


\title{
Session 3aAAa
}

\section{Architectural Acoustics: Case Studies and Design Approaches I}

\author{
Bryon Harrison, Cochair \\ 124 South Boulevard, Oak Park, IL, 60302 \\ Witew Jugo, Cochair \\ Institut für Technische Akustik, RWTH Aachen University, Neustrasse 50, 52066 Aachen, Germany
}

\section{Contributed Papers}

\begin{abstract}
8:00
3aAAa1. Acoustic and audience response analyses of eleven Venetian churches. Davide Bonsi (The Acoustics Lab., Fondazione Scuola di San Giorgio, Isola di San Giorgio Maggiore, I-30124 Venezia, Italy, davide.bonsi@scuoladisangiorgio.it), Malcolm Longair (Cavendish Lab., University of Cambridge, JJ Thomson Avenue, CB3 0HE Cambridge, UK, pjg47@cam.ac.uk), Philip Garsed (Dept. of Engineering, University of Cambridge, Trumpington Street, CB2 1PZ Cambridge, UK, ms11000@hermes.cam.ac.uk), Raf Orlowski (Arup Acoustics, St Giles Hall Pound Hill, CB3 0AE Cambridge, UK, Raf.Orlowski@arup.com)

A research project has been carried out by the University of Cambridge (UK), Arup Acoustics (Cambridge, UK) and the Fondazione Scuola di San Giorgio-CNR (Venice, Italy) with the purpose of investigating the relationship between music and the architectural spaces within which it was performed in the context of Venetian vocal polyphony during the 16th century. The most representative churches (San Marco, Parish churches, hospitals and monasteries) were selected for this study and the music was performed within them by the Choir of St John's College of Cambridge University. Members of the audience completed questionnaires assessing the acoustic qualities of the spaces. The results of the audience and expert scores were then statistically analyzed and correlated with a set of room acoustic indices which had been measured in the same locations. In the present paper technical aspects of the experiments and the data analysis will be illustrated. The principal conclusions were as follows: 1 . There is a strong correlation between the subjective impression of reverberance with EDT and T30 and musical clarity with C80;2. A grouping of the churches by typology was found by analysing the reverberation times; 3 . The poor quality of clarity was assessed for churches with longest EDT for the performance of music involving polyphony.
\end{abstract}

\section{8:20}

3aAAa2. A Comparative Study of Four Concert Halls in Istanbul: Correlation of Subjective Evaluation with Objective Acoustic Parameters. Nurgun Bayazit Tamer (Istanbul Technical University, Taskisla Taksim Istanbul, 34437 Istanbul, Turkey, nurgun@itu.edu.tr), Anil Vural (Istanbul Technical University, Taskisla Taksim Istanbul, 34437 Istanbul, Turkey, vuralanist@yahoo.com)

In the studies of room acoustics, the parameters that are taken into consideration while evaluating the acoustic performance of a hall are examined in two sections: objective acoustic parameters which can be measured and calculated and, subjective acoustic parameters which define the impacts of a hall's acoustical qualities over the audiences. During the first half of the century, the reverberation time was considered to be the most important objective acoustic parameter which defined the acoustic quality of a hall. But, the later studies indicated that this parameter wasn't sufficient alone to define a subjective evaluation. Consequently, studies have been made to define the subjective influence on people from the acoustic performance of a hall. In this study, it has been attempted to define the statistical correlation between the subjective evaluation of the acoustic characteristics of the four concert halls, which was done by the members of orchestra together with the ordinary concert-goers, and their objective acoustic parameters are explained.
The detailed objective acoustic parameters are presented for measurements in four concert halls in Istanbul. The halls are used by many different performances, from concerts to ballet or opera. The measured octave band quantities are RT, EDT, G,C80 and D50.

8:40

3aAAa3. Computer modelling and simulation of sound field in $\mathbf{2 4}$ rectangular concert halls. Andrzej K. Klosak (Institute of Building Materials and Structures, Faculty of Civil Eng., Cracow University of Technology, Warszawska 24, 31-155 Cracow, Poland, andrzej.klosak@pk.edu.pl)

Extensive acoustics computer simulations has been made using Odeon computer simulation software. From 300 to 850 measurements positions have been analysed in 24 rectangular rooms representing "shoe-box" type concert halls with volumes of $8000 \mathrm{~m}^{3}, 12000 \mathrm{~m}^{3}$ and $16000 \mathrm{~m}$. In total 14 000 receiver positions have been analysed. For each receiver position eight objective measures have been calculated, in particular Reverberation Time $\left(\mathrm{T}_{30}\right)$, Early Decay Time (EDT), Clarity $\left(\mathrm{C}_{80}\right)$, Strength $\left(\mathrm{G}, \mathrm{G}_{\text {early }}, \mathrm{G}_{\text {late }}\right)$, Early Lateral Energy Fraction $\left(\mathrm{LF}_{80}\right)$ and Late Lateral Strength $\left(\mathrm{G}_{\mathrm{LL}}\right)$. Most of measures have been calculated in six octave bands from $125 \mathrm{~Hz}$ to $4000 \mathrm{~Hz}$. Extensive amount of generated data required developing of new methods for processing, comparing and graphical presentation of results. Those methods together with description of modelling and simulation procedures are described in this paper.

\section{9:00}

3aAAa4. Improvement of acoustic conditions in a historical interior by means of a DSP-controlled sound-reinforcement system. Tadeusz Fidecki (F. Chopin Academy of Music, Okolnik 2, 00-368 Warszawa, Poland, fidecki@chopin.edu.pl), Andrzej Miskiewicz (F. Chopin Academy of Music, Okolnik 2, 00-368 Warszawa, Poland, misk@chopin.edu.pl), Jan Zera (Centr. Inst. for Labour Prot. - Natl. Res. Inst., Czerniakowska 16, 00701 Warsaw, Poland, jazer@ciop.pl)

Stringent building conservation requirements in historical interiors pose substantial limitations in acoustical treatment and sound reinforcement systems used to secure proper sound quality. This presentation discusses the means undertaken to improve the acoustic conditions in the historic Great Ballroom of the Royal Castle in Warsaw. Originally, the room was too reverberant $\left(\mathrm{T}_{30}=2.9 \mathrm{~s}\right.$ at $\left.500 \mathrm{~Hz}\right)$ what resulted in a lack of clarity of performed music and poor speech intelligibility (STI $<0.3$ ). A precise laser scan of the interior was made to obtain input data for a computer acoustic model of the room. This model was used for selection of a DSP-controlled sound reinforcement system to improve listening conditions for speech and music. [Work supported by the Polish Ministry of Science and Higher Education, grant No. R17 004 02]. 


\section{9:20}

3aAAa5. On the acoustics of a specifically designed library discussion room with corrugated ceiling. Nico F. Declercq (Georgia Tech Lorraine G.W. Woodruff School of ME, UMI Georgia Tech - CNRS 2958, 2 rue Marconi, 57070 Metz, France, nico.declercq@me.gatech.edu), Katrien Dewijngaert (Georgia Tech Lorraine - G.W. Woodruff School of ME, UMI Georgia Tech - CNRS 2958, 2 rue Marconi, 57070 Metz, France, declercq@ieee.org), Katelijn Vanderhaeghe (Georgia Tech Lorraine - G.W. Woodruff School of ME, UMI Georgia Tech - CNRS 2958, 2 rue Marconi, 57070 Metz, France, declercq@ieee.org), Patricia Verleysen (Ghent University, Mechanics of Materials and Structures, Sint-Pietersnieuwstraat 41, 9000 Ghent, Belgium, Patricia.Verleysen@ugent.be)

There is a discussion room at the library of Viipuri in Finland where the ceiling has been especially designed to enhance the acoustics and make every position within the room acoustically equivalent. In other words no matter where a speaker is standing, he is supposed to be heard equally well all over the room. For that purpose a corrugated ceiling has been constructed that must distribute sound optimally, at least from the point of view of a ray theoretical analysis. The numerical study presented here shows that the corrugation is such that the ray approach is not exactly valid and a plane wave expansion technique shows that the acoustics is much worse than it is supposed to be. A detailed description of the sound distribution as a function of the position of the sound source and the receiver is presented. The acquired knowledge is important for future construction of similar rooms.

\section{9:40}

3aAAa6. Acoustic climate inside a canteen and mitigation solutions Giuseppe Marsico (APAT, Via Vitaliano Brancati 48, 00144 Rome, Italy, giuseppe.marsico@apat.it), Giovanni Brambilla (CNR Institute of Acoustics, Via del Fosso del Cavaliere 100, 00133 Rome, Italy, giovanni.brambilla@idac.rm.cnr.it), Salvatore Curcuruto (APAT, Via Vitaliano Brancati 48, 00144 Rome, Italy, salvatore.curcuruto @apat.it), Monica Clapiz (Via Edoardo Jenner 102, 00151 Rome, Italy, mony.c@libero.it), Rinaldo Betti (APAT, Via Vitaliano Brancati 48, 00144 Rome, Italy, rinaldo.betti@apat.it), Michele Riccardi (APAT, Via Vitaliano Brancati 48, 00144 Rome, Italy, michele.riccardi@apat.it)

The acoustic environment inside a canteen is an important feature to guarantee speech intelligibility and speech privacy, as well as to reduce the exposure levels. Unfortunately this aspect is not always properly taken into account as others, like cleanliness. This paper describes the case-study of the canteen at the National Agency for Environmental Protection (APAT), having a capacity of 220 seats. The acoustic environment has been characterized by measuring the main acoustic descriptors for speech intelligibility (Reverberation Time, STI, C80 and D50) and evaluating the noise exposure levels for the users. The comparison of the results with the recommended values showed a bad situation, especially in the speech frequency range, which requires acoustic corrections to improve the quality of the acoustic environment. To identify these corrections a numerical model of room acoustics, implemented on a commercial software, has been used to evaluate the effects produced by different corrections. The proposed correction improves the acoustic performances up to a good level, without modifying the structural parts and existing walls. Moreover, the solution allows to maintain the hall's usability without obstructing user mobility, screening natural lighting and reducing number of seats.

\section{0:00}

3aAAa7. Cost Benefit Analysis of Acoustic Treatments for Entertainment Venues. David M. Borgeaud (Environmental Resources Management Australia Pty Ltd, Level1, 60 Leichhardt Street, Spring Hill QLD, 4000 Brisbane, Australia, david.borgeaud@erm.com)

Fortitude Valley, in Brisbane City, Australia is an inner city area combining retail, commercial, residential, and entertainment uses. It has been the starting point for several major Australian bands. To address the increase in apartments in the Valley, the Brisbane City Council has developed a noise policy (called The Valley Music Harmony Plan) using entertainment precincts with specific noise criteria within the Valley, to allow informed decisions to be made regarding future development while minimising potential noise conflicts. An earlier paper by the Author has addressed the ambient noise mapping undertaken for the Valley, and this paper discusses the results of the cost benefit study undertaken into the acoustic treatment of selected venues. The purpose of this work was to provide Council with information on both the feasibility of treating the entertainment venues to reduce noise emissions and the order of cost for the treatments. The study of four venues found that attenuation of $3-22 \mathrm{~dB}(\mathrm{~A})$ or $1-17 \mathrm{~dB}(\mathrm{C})$ could be achieved with asociated costs ranging from $\$ 30,000$ to over $\$ 400,000$ (Australian dollars). The costing work provided invaluable knowledge in the development of the Valley Music Harmony Plan.

\section{0:20-10:40 Break}

\section{0:40}

3aAAa8. Open-air theatre in the centre of the city: Acoustic design and noise environment control. Nicolaos Barkas (Democritus University of Thrace/ Greece, Vas. Sofias street 1, Department of Architecture, 67100 Xanthi, Greece,nbarkas@arch.duth.gr)

The municipal open-air Garden Theatre of Thessaloniki has for decades been located in the biggest urban park of the city next to a busy crossroads, where five major traffic roads meet. The intensification of traffic noise over the years led to the degradation of its use. In 1997, the organisation of the Cultural Capital of Europe served as a springboard for a study conducted by the author on redesigning the theatre. The design comprised a variety of arrangements, such as the rotation of the auditorium according to the results of the noise environment study, the choice of a closed plan in the type of the Greek-Roman Odeion, the sinking of the theatre space and the construction of barriers and shades. Various problems (such as archaeological findings, existence of big trees inside the auditorium, high level of underground water) and budget cuts during the design and construction, led to restricted execution of the planned architectural interventions. This paper deals with the design parameters that optimise acoustic function and maximise sound protection in an urban, open-air theatre and includes an analysis of the theatre's initial state, acoustic proposals and an assessment of the final applications.

\section{1:00}

3aAAa9. New acoustic test chambers at the University of Palermo. Marco Gullo (DREAM, Univ. of Palermo, Viale delle Scienze, Edificio 9, 90128 Palermo, Italy, mgullo@dream.unipa.it), Armando La Pica (DREAM, Univ. of Palermo, Viale delle Scienze, Edificio 9, 90128 Palermo, Italy, lapica@unipa.it), Giuseppe Rodono' (DREAM, Univ. of Palermo, Viale delle Scienze, Edificio 9, 90128 Palermo, Italy, rodon@unipa.it), Vincenzo Vinci (DREAM, Univ. of Palermo, Viale delle Scienze, Edificio 9, 90128 Palermo, Italy, vincenzo_vinci @ hotmail.it), Vincenzo Conti (DREAM, Univ. of Palermo, Viale delle Scienze, Edificio 9, 90128 Palermo, Italy, conti@csai.unipa.it)

Acoustic test chambers at the University of Palermo have been completed after a design optimization of existent building structures. A unique building houses five reverberation chambers, differently coupled each other in order to achieve a large flexibility on testing procedures, and offices for the staff. Main measurements offered to national and international standards include determination of sound power, sound insulation and sound absorption. Reverberation chambers present peculiar geometries in order to achieve the desidered sound fields. Chambers structure and layout within the whole building, housing all the acoustic facilities, has been designed in order to achieve strong sound insulation requirements. The present work describes design solutions and reportes preliminary results from a large, still running, validation in order to characterize the chamber acoustic behaviour. Design targets and objective achievements are listed and commented.

\section{1:20}

3aAAa10. Design and construction of an acoustic lab facility. Douglas Winker (ETS-Lindgren, 1301 Arrow Point Drive, Cedar Park, TX 78763, USA, douglas.winker@ets-lindgren.com)

This paper will discuss the design and construction of a new acoustic lab facility. The lab consists of a hemi anechoic chamber and a reverberation chamber suite. The hemi anechoic chamber was designed to obtain a $0 \mathrm{dBA}$ 
noise floor in the frequency range of interest. It features a 200 cubic meter volume inside the wedges. To achieve the design goals, several aspects were considered including ambient levels, vibration isolation, HVAC noise, and free field performance. The design solutions for each of these areas will be presented. The reverb chamber suite consists of two reverberation chambers designed to perform transmission loss tests and sound absorption testing. The source chamber has an internal volume of 214 cubic meters and the receive room has an internal volume of 418 cubic meters. Design considerations will be discussed including vibration isolation, wall panel design, and diffuser placement. Additionally, the design and construction of the transmission loss coupling will be addressed.

\section{$11: 40$}

3aAAa11. Measurements of existing modular construction classroom acoustics in the Midwestern United States. Norman Philipp (University of Nebraska - Lincoln, 1110 S. 67th St., Omaha, NE 68182-0681, USA, nphilipp@mail.unomaha.edu), Lily M. Wang (University of Nebraska Lincoln, 1110 S. 67th St., Omaha, NE 68182-0681, USA, LWang4@UNL .edu)

Modular construction classrooms in the United States currently total more than 300,000 units, with $37 \%$ nationally of all public schools utilizing portable classrooms, according to the Modular Building Institute and a 2007 survey of Public School Principals conducted by the National Center for Education Statistics. Given this ever growing number there is a desire to improve their acoustics to meet ANSI S12.60-2002 guidelines for classroom acoustics. This paper gives a survey of existing acoustic conditions of relocatable classrooms currently in use in the Midwestern United States. Data to be presented include reverberation times, interior/exterior noise reduction, and site noise measurements. The results of these measurements and additional qualitative surveys will aid in understanding the performance of modular classrooms and assist in drafting a standard for them to supplement the guidelines in ANSI S12.60-2002.

\section{2:00}

3aAAa12. The influence of the scattering coefficient on the reverberation time. Franz Huber (Ziviltechnikerbüro Dipl. - Ing. Franz Huber, Neumühl 44, A-3250 Wieselburg, Austria, fh@zthuber.at), Thomas Bednar (University of Technology, Karlsplatz 13/206, A-1040 Vienna, Austria, thomas.bednar@tuwien.ac.at)

The reverberation time is still seen as an important measure of quality in architectural acoustic. The absorption coefficient is to be considered as the largest influencing factor on the reverberation time. The formulas after Sabine and Eyring give us the opportunity to calculate the reverberation time, when the absorption coefficients are known. In contrast to this, is the investigation of the influence of the scattering coefficient on the reverberation time a relatively new field for research. In this study the reverberation time for a "shoebox" was simulated, where the parameters were the values of the absorptions coefficient and the scattering coefficient and the even and uneven distribution of the absorption coefficient. The results of the simulation were compared with the results from the formulas by Sabine and Eyring. The simulations indicate a significant influence of the scattering coefficient on the reverberation time especially at low absorption coefficients. You can see by comparing the results of the simulation with the results of the formulas by Sabine and Eyring, that the theoretical formulas assume a minimum of scattering coefficient. It shows that the variation of the scattering coefficient is a possibly way to influence the reverberation time.

\section{2:20}

3aAAa13. Air Absorption Error in Room Acoustical Modeling. Remy H. Wenmaekers (LeVeL Acoustics BV, De Rondom 10, 5612 AP Eindhoven, Netherlands, r.h.c.wenmaekers@tue.nl), Constant C. Hak (Technische Universteit Eindhoven, De Rondom 10, 5612 AP Eindhoven, Netherlands, c.hak@inter.nl.net), Heiko J. Martin (Eindhoven University of Technology, Den Dolech 2, BWK/BPS, 5600 MB Eindhoven, Netherlands, h.j.martin@tue.nl), Laurentius C. Van Luxemburg (LeVeL Acoustics BV, De Rondom 10, 5612 AP Eindhoven, Netherlands, 1.c.j.v .luxemburg@tue.nl)

Most statistical and ray-tracing computer models take into account the absorption of sound by air when calculating the reverberation time. Extensive research by many scientist lead to the standardized calculation model for pure tone air absorption. The phenomenon was discovered from a room acoustical point of view by Sabine, while the further development of the calculation model took place in the fields of physics and environmental noise. As a result, several parameters and units are used for the same phenomenon. However, air absorption is calculated for pure tones, while room acoustics calculations are performed for frequency bands. Most computer models use the center frequency of the normalized frequency bands to calculate the air absorption by the pure tone method. Frequency band reverberation measurements under laboratory and practical conditions show that errors larger than the JND are made in calculating the air absorption by this 'center frequency method'. No literature is found that provides an accurate air absorption calculation for frequency bands. 


\title{
Session $3 \mathbf{a A A b}$
}

\section{Architectural Acoustics: Prediction Methods in Building Acoustics I}

\author{
Berndt Zeitler, Cochair \\ NRC - Institute for Research in Construction, 1200 Montreal Road, Building M-27, Ottawa, ON K1A OR6, Canada \\ Catherine Guigou-Carter, Cochair \\ CSTB, 24, rue Joseph Fourier, Saint Martin D'Hères, 38400, France
}

\section{Contributed Papers}

\begin{abstract}
8:00
3aAAb1. FEM based prediction model for the impact sound level of floors. Andreas Rabold (Technische Universität München, Fakultät für Bauingenieur- und Vermessungswesen, Arcisstraße 21, 80290 München, Germany, rabold@bv.tum.de), Alexander Düster (Technische Universität München, Fakultät für Bauingenieur- und Vermessungswesen, Arcisstraße 21, 80290 München, Germany, duester@bv.tum.de), Ernst Rank (Technische Universität München, Fakultät für Bauingenieur- und Vermessungswesen, Arcisstraße 21, 80290 München, Germany, rank@bv .tum.de)
\end{abstract}

Up to now the research and development in the field of building acoustics is based mainly on experiments. The consequence of this approach is that the development and optimization of a new building component is a very tedious and expensive task. A considerably reduction of these costs could be achieved, if the optimization relying on experiments would be replaced - at least to some extent - by a computational approach. Common prediction models of the impact sound level from lightweight floors are based on measured impact sound level data for the component parts under consideration. These models are very useful for the evidence of performance of known building components, but they are not suited for the development of new components. An alternative approach in this context is the application of the finite element method (FEM). Based on a FE model, a modaland spectral analyses provides the framework for an optimization of the building component. This contribution will present the overall approach consisting of the three-dimensional modeling of a lightweight-floor and the excitation source (tapping machine), the subsequent modal- and the spectralanalyses and the computation of the radiated sound from the floor.

\section{$8: 20$}

3aAAb2. Human footsteps induced floor vibration. Delphine Bard (Lund University, Division of Engineering Acoustics, John Ericsson väg 1, 22100 Lund, Sweden, delphine.bard@acoustics.lth.se), Julia Sonnerup (Lund University, Division of Structural Mechanics, John Ericsson väg 1, 22100 Lund, Sweden, julia.sonnerup@byggmek.lth.se), Göran Sandberg (Lund University, Division of Structural Mechanics, John Ericsson väg 1, 22100 Lund, Sweden, goran@byggmek.lth.se)

The generation of the impact sound by the act of the human walk involves two factors, the characters of the footfall and the shape of the induced floor deflection. The footfall noise is created by the impact excitation and the characters of the footfall depend on the foot-ware: the heels and the frequencies of the footfall. The shape of the floor deflection depends rather on the geometrical walking pattern and the construction of the floor structure. In this investigation, the vibration patter of the light-weight construction floor is created by the same walking object, a male with common height. The excitation from the person to the floor in the FE simulations is a function of the length of the foot and the weight of the walking object. The geometrical time history is of the foot step allows it to have different directions in the room. Since the excitation is assumed to be deterministic, differences between the excitation frequencies are estimated from the video records. The goal of this investigation is to determine the difference of the floor structure deflections between two different walking paths: one is perpendicular to the bearing beams and the other is the diagonal path.

\section{Invited Papers}

\section{8:40}

3aAAb3. Vibration of a rib-reinforced floor/ceiling structures with irregularities. Hyuck Chung (The University of Auckland, Department of Mathematics, Private Bag 92019, 1001 Auckland, New Zealand, hyuck@ math.auckland.ac.nz), Colin Fox (Department of Physics, The University of Otago, 730 Cumberland Street, P O Box 56, 9010 Dunedin, New Zealand, fox @physics.otago.ac.nz)

This paper describes mathematical modelling procedure of the rib-reinforced floor/ceiling structures, which are made up of components with irregular shapes and physical parameters. Exact determination of the vibration of a composite structure becomes impossible beyond the low-frequency range because one cannot determine all the necessary parameters of the components. Even if every possible parameter of the structure is known, the results from such deterministic model would not represent the real behaviour of the structure. Therefore, the prediction model in the mid- to high-frequency range must include the effects of the irregularities. We show how the power spectra of the irregular features of each component can be included in the model. The model gives statistical estimates of the solutions, which can give appropriate mean and variance of the vibration of the structure for the given severity of the irregularities.

\section{9:00}

3aAAb4. Parameter survey of a rib stiffened wooden floor using sinus modes model. Lars-Göran Sjökvist (Acoustic Technology Department, Technical University of Denmark, Ørsted Plads, B352, DK-2800 Lyngby, Denmark, lars-goran.sjokvist@sp.se), Jonas Brunskog (Dept. of Acoustic Technology, Technical University of Denmark, Building 352, DK 2800 Lyngby, Denmark, jbr@oersted.dtu.dk), Finn Jacobsen (Acoustic Technology Department, Technical University of Denmark, Ørsted Plads, B352, DK2800 Lyngby, Denmark, fja@ oersted.dtu.dk)

In buildings built with new techniques there exists a need for better understanding of their acoustical performance. The development of large wooden houses slows down by the uncertainty and costly testing that have to be performed many times before gaining good 
results. A greater understanding of the sound insulation for lightweight buildings have the possibility to speed up the development of new techniques and in the end give tenants better quality of life. This study uses Fourier sinus series to calculate the vibrations on a rib stiffened plate. The beams are modelled as line forces and moments that reacts onto the plate vibrations. A parameter study is performed with the aim to identify the most important parameters and their behaviour. The preliminary results show that the attenuation of the system is by far most evident in the direction across the beams. The influence from the basic input parameters on the attenuation is then studied. And it is preliminary shown that the placement of the excitation force within a bay actually is irrelevant for the calculated attenuation.

\section{9:20}

3aAAb5. The modelling of vibration transmission through plate/beam structures typical of lightweight buildings. Laurent Galbrun (Heriot-Watt University, School of the Built Environment, EH14 4AS Edinburgh, UK, 1.g.u.galbrun@hw.ac.uk)

Vibration transmission through plate/beam structures typical of lightweight buildings is examined in this paper. Modelling difficulties are analysed and related to the several modelling choices available, including connection type, beam modelling, modal properties and material properties. A number of key experiments carried out on structures made of one or more parallel plates attached to a single beam are discussed, and the applicability and limitations of fundamental theories are identified through the use of Statistical Energy Analysis (SEA) models. It is shown that the interrelation between the various modelling factors can lead to complex models. The systems for which existing models can be used are identified, as well as those for which more complex models need to be developed.

\section{Contributed Papers}

9:40

3aAAb6. Approximation of single equivalent mobilities of timber joist floors. Andreas R. Mayr (Stuttgart University of Applied Sciences, Schellingstrasse 24, 70174 Stuttgart, Germany, andreas.mayr@hft-stuttgart .de), Barry M. Gibbs (University of Liverpool, School of Architecture, Abercromby Square, L693BX Liverpool, UK, bmg@liv.ac.uk)

To estimate the structure-borne sound power of vibrational active machines or components two source quantities, corresponding to the source activity and mobility, and one receiver quantity, the mobility, are usually required. A practical, laboratory-based measurement procedure for the characterisation of mechanical installations has recently been proposed, which yields single equivalent values of source strength and source mobility. In order to predict the power in the installed condition, an estimate of the receiver mobility also is required, again, for practical reasons, in the form of a single equivalent value. This paper considers the single equivalent receiver mobilities for lightweight building structures - timber joist floors. The value obtained is in the form of the average magnitude of the effective mobilities over the contact points. A simple prediction method is proposed for pointand transfer mobilities (both are required to yield the effective mobility at each contact) based on infinite beam and infinite plate behaviour. Estimates for point-, transfer- and single equivalent receiver mobilities are discussed and compared with measured data.

\section{0:00}

3aAAb7. Towards a prediction of the sound transmission from lightweight stairs. Jochen Scheck (Stuttgart University of Applied Sciences, Schellingstrasse 24, 70174 Stuttgart, Germany, jochen.scheck @ hft-stuttgart.de), Barry M. Gibbs (University of Liverpool, School of Architecture, Abercromby Square, L693BX Liverpool, UK, bmg@liv. ac.uk), Andreas Drechsler (University of Applied Sciences, Schellingstr. 24, 70174 Stuttgart, Germany, andreas.drechsler@hft-stuttgart .de), Heinz-Martin Fischer (University of Applied Sciences, Schellingstr. 24, 70174 Stuttgart, Germany, heinz-martin.fischer@hft-stuttgart.de)

The sound transmission from lightweight stairs which are connected to separating walls often gives rise for complaints. One reason for this is that at present there is no prediction method available. Treating the stair as an ac- tive component in a similar manner like vibrating machines stairs can be characterised as structure-borne sound sources. The source data then can be used to predict the sound transmission in buildings using parts of EN 12354. Following this approach investigations on a timber stair have been carried out in a staircase test facility. Based on a full characterisation by contact free velocity and mobility and in-situ measurement using an indirect method, more practical methods like the reception plate method and a characterisation based on a reference power calibration are investigated. The source data obtained was used to predict the sound transmission in buildings.

\section{0:20}

3aAAb8. Prediction of the impact sound transmission from lightweight stairs in buildings based on a simplified laboratory characterisation. Andreas Drechsler (University of Applied Sciences, Schellingstr. 24, 70174 Stuttgart, Germany, andreas.drechsler@hft-stuttgart.de), Jochen Scheck (Stuttgart University of Applied Sciences, Schellingstrasse 24, 70174 Stuttgart, Germany, jochen.scheck@hft-stuttgart.de), Heinz-Martin Fischer (University of Applied Sciences, Schellingstr. 24, 70174 Stuttgart, Germany, heinz-martin.fischer@hft-stuttgart.de)

Based on a simplified laboratory characterisation of lightweight stairs the normalized impact sound pressure level in buildings can be predicted using parts of EN 12354. For the characterisation an approach is followed where the stair is treated as an active component with respect to an external excitation (e.g. by the tapping machine). The laboratory characterisation was performed for a timber stair and the normalized impact sound pressure level was predicted for different building situations. The various transmission paths via the separating and flanking walls were investigated and compared to the prediction according to EN 12354. Within the presentation the proceeding will be outlined and the restrictions are discussed. By comparison of measured and calculated values some references for the accuracy of the method are obtained.

10:40-11:00 Break 


\section{Invited Paper}

11:00

3aAAb9. Impedance of standard impact sources and their effect on impact sound pressure level of floors. Berndt Zeitler (NRC - Institute for Research in Construction, 1200 Montreal Road, Building M-27, Ottawa, ON K1A 0R6, Canada, berndt .zeitler@nrc-cnrc.gc.ca), Trevor Nightingale (NRC - Institute for Research in Construction, 1200 Montreal Road, Building M-27, Ottawa, ON K1A 0R6, Canada, Trevor.Nightingale@nrc-cnrc.gc.ca)

This paper reports on a systematic study of the Impact Sound Pressure Level (ISPL) due to different standard sources (ISO hammer box, Ball, and "Bang Machine") when applied to a wood-joist floor-ceiling construction with and without various floor toppings. Measurement data show that for each floor, the ISPL was different for each source. Not surprising since the blocked force for each source is different. But, after applying corrections for the applied force the ISPL's remain different, suggesting problems since the sources simulate the same human walking activity. Perhaps more importantly, the rank of topping effectiveness (which is simply the difference in ISPL with and without the topping) was different for the three sources. Measurements of the drive point impedance of sources and floors are presented to explain this behaviour and to recognise that the power injected is not just related to the applied force, but also a function the impedance match (or miss-match) between the source and floor. The paper shows using a simple model, it is possible to predict ISPL's for any source if the forces and impedances are known, and using this model floor treatment effectiveness can be correctly ranked by each of the three standardised sources.

\section{Contributed Papers}

\section{1:20}

3aAAb10. Conversion of floor impact sound levels in apartment buildings. Pyoung Jik Lee (Hanyang University, Department of Architectural Engineering, 133-791 Seoul, Republic of Korea, pyoungjik@daum .net), Shin-Ichi Sato (Hanyang University, Department of Architectural Engineering, 133-791 Seoul, Republic of Korea, s_sato@mac.com), Jin Yong Jeon (Hanyang University, Department of Architectural Engineering, 133791 Seoul, Republic of Korea, jyjeon@hanyang.ac.kr)

A procedure to convert the floor impact sound level of the bang machine as well as the tapping machine into that of the impact ball was suggested. Because the bang machine has been the only standard heavy-weight impact source for a long time in Japan and Korea, much of its data is available. The conversion factor of the sound pressure level between the impact ball and other standard impact sources was applied to the sound pressure level of a bang machine and a tapping machine. In addition, a conversion method using a correlation analysis between the sound pressure level of impact ball and that of other standard impact sources at each octave band was also investigated. Results indicate that bang machine and tapping machine data can be successfully converted into impact-ball data, in the case of boxframed type, reinforced concrete structures.

\section{1:40}

3aAAb11. A simplified approach to impact sound insulation rating without using a tapping machine. Ming Li (Acoustics Research Centre, School of Architecture, The University of Auckland, 1010 Auckland, New Zealand, mli072@ec.auckland.ac.nz), George Dodd (Acoustics Research Centre, School of Architecture, The University of Auckland, 1010 Auckland, New Zealand, mli072@ec.auckland.ac.nz)

The aim of this study is to investigate a simplified approach for obtaining ratings of impact insulation of floors which requires only airborne sound measurements and correction factors depending on the particular type of floor finish. One third octave frequency-dependent correction factors have been obtained by processing published results of both airborne and impact insulation on floors. The uncertainty of predicted Ln,w values using correction factors shows a good agreement with ISO recommended limits. The proposed technique has value in reducing the cost and effort for field insulation measurements. Further work is underway to refine the method and to extend the range of constructions for which we have correction factor.

\section{2:00}

3aAAb12. Reciprocity and the Prediction of the Apparent Sound Reduction Index for Lightweight Structures According to EN12354. Jeffrey Mahn (University of Canterbury, Department of Mechanical Engineering, Private Bag 4800, 8140 Christchurch, New Zealand, jma251 @ student.canterbury.ac.nz), John Pearse (University of Canterbury, Depart-ment of Mechanical Engineering, Private Bag 4800, 8140 Christchurch, New Zealand, john.pearse@canterbury.ac.nz)

EN12354 makes use of reciprocity to cancel the radiation efficiency terms from the equations used to predict the apparent sound reduction index. The use of reciprocity works well for the application of EN12354 to massive, concrete structures with diffuse wave fields and with critical frequencies below the frequency range of interest. However, lightweight constructions may have critical frequencies above the frequency range of interest and may not support diffuse wave fields. The use of reciprocity for application to lightweight structures may introduce errors into the predictions. This study investigates the uncertainty introduced into the predictions of the apparent sound reduction index of lightweight constructions due to the use of reciprocity.

\section{2:20}

3aAAb13. Innovative building systems to improve the acoustical quality in lightweight masonry constructions: Application of resilient joints at junctions - PART 1: Analysis of the experimental results. Charlotte Crispin (BBRI, rue du Lombard, 42, B-1000 Brussel, Belgium, charlotte.crispin@bbri.be), Bart Ingelaere (BBRI, rue du Lombard, 42, B-1000 Brussel, Belgium, bart.ingelaere@bbri.be), Gerrit Vermeir (Lab. ATF, Katholieke Universiteit Leuven, Celestijnenlaan 200D, B-3001 Leuven, Belgium, Gerrit.Vermeir@bwk.kuleuven.be)

The more severe acoustic requirements imposed by the new Belgian standard for dwellings are a real challenge for the building professionals (architects, contractors, building elements manufacturers and suppliers, œ) and more particularly for the market of lightweight materials. An important brick producer in partnership with the BBRI has succeeded to propose efficient acoustic solutions for these kinds of materials by treating in particular the flanking transmission. Indeed, to obtain high sound insulations, the structural transmission paths of noise through the flanking walls cannot be neglected any more. By the application of resilient rubber interlayers at the junctions, these transmission paths are nearly eliminated. A large number of measurements was carried out in order to study in detail the effect of these flexible joints on the sound transmission. These measurements were made in a specially designed laboratory where vibration reduction indexes can be measured for all types of connections and for different loads. We present, in this paper, the measurement campaign and the analysis of the results. 


\section{Contributed Paper}

$1: 40$

3aAAb14. Innovative building systems to improve the acoustical quality in lightweight masonry constructions: Application of resilient joints at junctions - PART 2: Study cases modelled according to the standard 12354-1 (2000). Charlotte Crispin (BBRI, rue du Lombard, 42, B-1000 Brussel, Belgium, charlotte.crispin@bbri.be), Bart Ingelaere (BBRI, rue du Lombard, 42, B-1000 Brussel, Belgium, bart.ingelaere@bbri.be), Debby Wuyts (BBRI, rue du Lombard, 42, B-1000 Brussel, Belgium, debby.wuyts@bbri.be)

An important brick manufacturer in partnership with the BBRI has suc- ceeded to propose efficient acoustic solutions for this kind of lightweight materials by treating the flanking transmission using flexible interlayers at junctions and foundations. A large measurement campaign has been carried out to study the efficiency of the resilient joint and to provide a prediction model on airborne sound insulation in brickwork constructions. The prediction model used is based on the standard 12354-1 but was adapted in order to take into account higher orders for the flanking transmission paths. This adapted model thus allowed to study different applications of the flexible joint and their contribution to the improvement of the global sound insulation. This paper presents a discussion on the calculation model, the input data and some early results.

\section{Invited Papers}

3aAAb15. Direct and flanking sound transmission of solid gypsum walls - practical experience and numerical prediction. Andreas Ruff (Stuttgart University of Applied Sciences, Schellingstrasse 24, 70174 Stuttgart, Germany, andreas.ruff @ hft-stuttgart.de), Heinz-Martin Fischer (University of Applied Sciences, Schellingstr. 24, 70174 Stuttgart, Germany, heinz-martin .fischer@hft-stuttgart.de)

In modern multi storey buildings gypsum blocks are often used for solid inner walls without static requirements. However, these relatively light walls with a mass per unit area of about $90 \mathrm{~kg} / \mathrm{m}^{2}$ are not connected rigidly to the adjacent building elements. They are decoupled with elastic interlayers made of bitumen, cork or polyethylene foam. These elastic interlayers have a significant influence on the direct and the flanking sound transmission of the gypsum walls. For this reason the decoupled walls have to be considered different to rigidly connected walls. Within a recent research project there was an extensive investigation into the direct and the flanking sound transmission of gypsum walls in different test facilities in the laboratory. For several kinds of elastic interlayers the sound reduction index of gypsum walls was measured in the test stand for direct sound transmission. The vibration reduction index was investigated in a test facility for flanking transmission as well as in different building situations. The measured values are used as input data for the prediction model according EN 12354-1. The overall sound reduction index calculated with this prediction model is compared with the results measured in the buildings.

$$
\text { 2:20 }
$$

3aAAb16. Some practical aspects of the prediction of structure-borne sound caused by house-hold equipment. Eddy Gerretsen (TNO Science and Industry, Stieljesweg 1, 2628CK Delft, Netherlands, eddy.gerretsen@tno.nl)

Sound levels in rooms due to service equipment in the building can be an important reason for disturbance and more so in light weight building structures. Though the recently published draft standard prEN 12354-5 gives a framework for the prediction of the structure-borne sound as caused by this type of sources, there is still a lot to be studied and developed. That is certainly the case for light weight building structures. Some possibilities to simplify the indicated models have been studied and experimental data has been gathered for house hold equipment, taking a washing machine as an example, applied both in a 'heavy' and in a 'light weight' building. Various possibilities to apply substitution methods to characterise such a source as structure-borne sound source have been tried and compared.

\section{Contributed Papers}

\section{2:40}

3aAAb17. An hybrid method for sound transmission through opened windows. Philippe A. Jean (CSTB, 24 rue Joseph Fourier, 38400 SaintMartin-d'Hères, France, philippe.jean@cstb.fr), Michel Villot (CSTB, 24 rue Joseph Fourier, 38400 Saint-Martin-d’Hères, France, michel.villot@ cstb .fr)

An hybrid model for the calculation of sound transmission between apartments when windows are opened is presented. It is an extension of Kropp's model for balconies (integral/modal/wave approach). Two rectangular rooms are set in a baffle with rectangular openings. A point source is placed in one room and sound attentation between rooms is computed. The external field may include the ground and a nearby building by means of a combined image source approach to compute a Green function in the integral description at the openings. Results are validated against BEM and published measurements. SEA is employed to assess the importance of vibration transmission relative to airborne transmission through the external medium.

\section{3:00}

3aAAb18. Directional distribution of acoustic energy density incident to a surface under reverberant condition. Cheol-Ho Jeong (Acoustic Technology, DTU Elektro, Technical University of Denmark, Building 352, Ørsted plads, DK-2800 Kgs. Lyngby, Denmark, chj@oersted.dtu .dk), Jeong-Guon Ih (KAIST, Dept. of Mechanical Engineering, Science Town, 305-701 Daejeon, Republic of Korea, J.G.Ih@kaist.ac.kr)

Most of acoustic measurements presume ideal conditions: one ideal condition is a diffuse and reverberant field. In a practical situation, diffuse sound field in a reverberation chamber can not be achieved. In particular, the directional diffusion which means the uniform angular distribution of incident direction can not be satisfied. Angle dependency of incident energy density was simulated by the phased beam tracing simulation. Various results were found by changing room shape and source position, and number of sources. Acoustic energy generally decreases with the angle of incidence. This result can be used as a weighting function for the calculation of sound transmission index and random incidence absorption coefficient. 


\title{
Invited Paper
}

$3: 20$

3aAAb19. Exposure of building roofs to road traffic noise; consequences on the field performance of skylights. Michel Villot (CSTB, 24 rue Joseph Fourier, 38400 Saint-Martin-d'Hères, France, michel.villot@cstb.fr), Philippe A. Jean (CSTB, 24 rue Joseph Fourier, 38400 Saint-Martin-d'Hères, France, philippe.jean@cstb.fr)

In this paper, the exposure to road traffic noise of building roofs is studied both theoretically and experimentally. First, the incident field is estimated using a BEM approach, taking into account the sound diffraction on the roof edge. The theoretical results show significant differences in noise exposure between roofs and vertical façades, particularly with relatively tall buildings; these differences are then confirmed by field measurements. The grazing incidence of the sound fields on roofs raises the question of the acoustic performances of skylights in real situation, as opposed to their laboratory performances measured under diffuse sound field; this problem is experimentally investigated by laboratory measurements of glazing under different angles of incidence, using a big size plane wave generator.

WEDNESDAY MORNING, 2 JULY 2008

ROOM 342B, 8:00 TO 10:00 A.M.

\section{Session 3aABa}

\section{Animal Bioacoustics, Underwater Acoustics, Acoustical Oceanography, Signal Processing in Acoustics, and ECUA: Animal Sonar Systems III}

\author{
James Simmons, Cochair \\ Brown University, Dept. of Neuroscience, Box G-LN, Providnece, RI 02912, USA \\ Michel Andre, Cochair \\ Laboratori, d'Aplicacions Bioacútques, Universitat Politècnica de Catalunya, avda. Rambla Exposicío s/n, 08800 Vilanova, \\ la Geltrú, Spain
}

\section{Contributed Papers}

\section{8:00}

3aABa1. Localization of brief sounds by a bottlenose dolphin. Gennadi L. Zaslavski (University Authority for Applied Research, RAMOT, TelAviv University, str. Gordon 51 app 7, 42442 Netanya, Israel, gennadi .zaslavski@gmail.com)

Spatial hearing in dolphins is believed to be similar to that in humans. The same binaural phenomenon known for humans is used to explain sound localization in dolphins. However, the ability of a bottlenose dolphin to localize a sound in the vertical plane as accurate as in the horizontal plane appears to undermine binaural phenomenon concept in dolphin sonar. We found that directionality of the bottlenose dolphin transmit beam could account for most results on the bottlenose dolphin underwater object localization. In this paper passive localization and spatial discrimination of brief signals by a bottlenose dolphin is examined. The bottlenose dolphin was found capable of discriminating clicks with different intensity as well as a click and brief noise pulse simultaneously transmitted via transducers separated in azimuth by less than $1^{\circ}$. The dolphin was also able to discriminate a lead from a lag noise pulse at the transducer azimuth separation as small as $0.3^{\circ}-0.4^{\circ}$ and the lag delay as small as 20 microseconds. The results are discussed with regard to the bottlenose dolphin auditory time resolution and so called precedence effect phenomenon well known for the binaural system of humans.

\section{8:20}

3aABa2. The interaction of emitted pulses and echoes in a whale's biosonar: A partial contribution to biosonar automatic gain control. Alexander Y. Supin (Institute of Ecology and Evolution, 33 Leninsky Prospect, 119071 Moscow, Russian Federation, alex_supin@mail.ru), Paul E. Nachtigall (University of Hawaii, Hawaii Institute of Marine Biology, P.O. Box 1106, Kailua, HI 96734, USA, nachtiga@ hawaii.edu), Marlee Breese (University of Hawaii, Hawaii Institute of Marine Biology, P.O. Box 1106, Kailua, HI 96734, USA, mbreese@ hawaii.edu)
In a false killer whale Pseudorca crassidens, echo perception thresholds were measured using a go/no-go psychophysical paradigm and one-up-onedown staircase procedure. Electronically synthesized echoes were triggered by whale-emitted echolocation pulses, and the echo amplitudes were presented proportional to the triggering biosonar pulse amplitudes, thus imitating the basic feature of real target echoes. Echo thresholds were measured in terms of attenuation relative to the source level of the biosonar pulses. The echo threshold dependence on echo delay was investigated. With increasing echo delay, the threshold echo attenuation decreased from $-49.3 \mathrm{~dB}$ at $2 \mathrm{~ms}$ to $-79.5 \mathrm{~dB}$ at $16 \mathrm{~ms}$, with a regression slope of $-31.5 \mathrm{~dB}$ per delay decade. At delays longer than $16 \mathrm{~ms}$, the threshold remained nearly constant around $-80.4 \mathrm{~dB}$. This echo threshold dependence on delay is interpreted as a release from forward masking by the preceding emitted pulse. This release may well compensate for the echo level decrease with distance which is from 20 to $40 \mathrm{~dB}$ per distance decade, depending on target properties. This compensation may keep the echo sensation level independent of, or little dependent on, target distance. Supported by Office of Naval Research (USA), Ministry of Science and Education (Russian Federation).

\section{8:40}

3aABa3. Investigation into the possible role of dolphins' teeth in sound reception. Sabine Graf (University of Bath, Dept. Mechanical Engineering, Claverton Down, BA2 7AY Bath, UK, sg283@bath.ac.uk), William M. Megill (University of Bath, Dept. Mechanical Engineering, Claverton Down, BA2 7AY Bath, UK, enswmm@bath.ac.uk), Philippe Blondel (University of Bath, Department of Physics, Claverton Down, BA2 7AY Bath, UK, pyspb@bath.ac.uk), Sally E. Clift (University of Bath,Dept. Mechanical Engineering, Claverton Down, BA2 7AY Bath, UK, enssec@bath.ac.uk)

Odontocetes use active sonars for echolocation, navigation and socialisation. These sonars are characterised by narrow transmission and reception directivity patterns, over a variety of ranges. There is physiological 
and behavioural evidence to suggest that dolphins hear the echoes of their high-frequency clicks through their lower jaws. Current theory suggests that sound is transmitted through a thin region at the base of the jaw into a waveguide leading to the ear. The angular precision predicted by this theory is however much less than dolphins have been observed behaviourally to be able to do. A novel hypothesis is that the teeth of the dolphin's lower jaw act as an end-fire sonar array. This paper will start by putting these competing hypotheses into their context, presenting bioacoustics in the ocean, dolphin echolocation physiology and acoustic behaviour, and the mechanical properties of bones and teeth. This information is used to model the reception of different sounds by bottlenose dolphins (tursiops truncatus) with each possible mechanism, investigating the variations with nerve delay, the role of the jaw relative to the teeth, and the resulting changes in frequency sensitivity and directivity. The results can then be compared with observations of behavioural patterns.

\section{9:00}

3aABa4. Acoustic behavior of echolocating porpoises during prey capture. Stacy Deruiter (Woods Hole Oceanographic Institution, Applied Ocean Physics \& Engineering Dept., Woods Hole, MA 02543, USA, deruiter@mit.edu), Alexander Bahr (MIT, 32 Vassar St., Room 32-331, Cambridge, MA 02139, USA, abahr@mit.edu), Marie-Anne Blanchet (Fjord\&Bælt / University of Southern Denmark, Margrethes Plads 1, 5300 Kerteminde, Denmark, marie@fjord-baelt.dk), Sabina Hansen (Fjord\&Bælt / University of Southern Denmark, Margrethes Plads 1, 5300 Kerteminde, Denmark, sabina@fjord-baelt.dk), Jakob Kristensen (Fjord\&Bælt / University of Southern Denmark, Margrethes Plads 1, 5300 Kerteminde, Denmark, jakob@fjord-baelt.dk), Peter T. Madsen (University of Aarhus, Biological Sciences, Zoophysiology, C. F. Møllers Allé, Building 1131, DK-8000 Aarhus, Denmark, peter.madsen @ biology.au.dk), Peter Tyack (Woods Hole Oceanographic Institution, Applied Ocean Physics \& Engineering Dept., Woods Hole, MA 02543, USA, ptyack@whoi.edu), Magnus Wahlberg (Fjord\&Bælt / University of Southern Denmark, Margrethes Plads 1, 5300 Kerteminde, Denmark, magnus @ fjord-baelt.dk)

Porpoise echolocation has been studied in target detection experiments using stationed animals and steel spheres as targets, but little is known about the acoustic behavior of free swimming porpoises as they echolocate for prey. Here we used archival tags (modified Dtags) recording audio (400 $\mathrm{kHz}, 16$-bit stereo sampling rate) and animal movements (depth, heading, 3D pitch and roll, and acceleration) to study echolocation in trained porpoises catching fish. The prey capture experiments were conducted with and without suction cups covering the porpoises' eyes. Without the eye cups, the porpoises could use both echolocation and vision to locate and ingest their prey, but with eye cups they could use only echolocation. Our data show that time to prey capture is about $27 \%$ greater with eye cups (T-test, $\mathrm{p}<0$ $.01)$. They also allow us to describe and compare prey capture in the two experimental conditions, including analysis of the animals' detailed movements and echolocation behavior leading up to and following prey capture events.

\section{9:20}

3aABa5. Biosonar model for obtaining fine target structure in complex targets. John R. Buck (UMass Dartmouth, ECE Dept, 285 Old Westport Rd, N. Dartmouth, MA 02740-2300, USA, johnbuck@ieee.org)

Bats use echolocation to discriminate targets with multiple glints in which the glints are much closer together in time than the integration time of the bat auditory system. This indicates the bats cannot be using purely timedomain processing to image the target. It is likely that the bats exploit cues such as spectral peaks and notches due to the interference between the glints at specific frequencies. However, these interference patterns quickly grow complicated as the number of glints increases. The cepstrum is a signal processing algorithm designed to identify these interference intervals. We previously demonstrated that simple two-glint targets can be accurately resolved using a time-varying version of the cepstrum, or cepstrogram. This talk will present an algorithm for interpreting the evolution of cepstral peaks across a target with many glints to estimate the inter-glint intervals. This algorithm has the desirable quality of processing the data causally as it is received, and thus is possible to implement in real time. The performance of the algorithm will be evaluated using experimental data from an ultrasonic bat simulator. [Work supported by US Office of Naval Research]

\section{Invited Paper}

\section{9:40}

3aABa6. Acoustics of shape recognition by a dolphin in a cross-modal matching-to-sample paradigm. Matthias Hoffmann-Kuhnt (Acoustic Research Laboratory, National University of Singapore, 12A Kent Ridge Road, 119223 Singapore, Singapore, mahoku@nus.edu.sg), Mandar A. Chitre (Acoustic Research Laboratory, National University of Singapore, 12A Kent Ridge Road, 119223 Singapore, Singapore, mandar@arl.nus.edu.sg), Paul J. Seekings (Acoustic Research Laboratory, National University of Singapore, 12A Kent Ridge Road, 119223 Singapore, Singapore, paul@arl.nus.edu.sg), Grant Abel (Ocean Park Hong Kong, Aberdeen, 118336 Hong Kong, Hong Kong, grant.abel@oceanpark.com.hk)

A pacific bottlenose dolphin was trained in a two-alternative cross-modal matching-to-sample paradigm. The animal was able to inspect complex PVC-pipe shapes through echolocation or vision but never through both senses simultaneously. Acoustic data was collected through a 3-channel high-frequency recording system while the dolphin performed one of the following tasks: (1) match a complex shape with its sonar only (pure echoic matching), (2) match from vision to echolocation and (3) match from echolocation to vision. Simultaneously, synchronized in-air and underwater video was recorded documenting the approach path of the dolphin to either the sample object or to the alternative objects. The collected data was analyzed for type of click signals used, the frequency range of the emitted clicks, number of clicks emitted before a successive match and variations of click type with different objects. 


\title{
Session $3 a A B b$
}

\section{Animal Bioacoustics and ECUA: Odontocete Acoustics I}

\author{
David K. Mellinger, Cochair \\ Oregon State Univ. and NOAA, 2030 SE Marine Science Dr., Newport, OR 97365, USA \\ Michel Andre, Cochair \\ Laboratori d'Aplicacions Bioacústiques (Universitat Politècnica de Catalunya), avda. Rambla Exposició s/n, Vilanova i la \\ Geltrú, 08800, Spain \\ Invited Paper \\ 10:20
}

\begin{abstract}
3aABb1. Annotated odontocete recordings for developing automated detection and classification methods. David K. Mellinger (Oregon State Univ. and NOAA, 2030 SE Marine Science Dr., Newport, OR 97365, USA, David.Mellinger@ oregonstate.edu), Holger Klinck (Oregon State Univ. and NOAA, 2030 SE Marine Science Dr., Newport, OR 97365, USA, Holger.Klinck@oregonstate .edu), Sara Heimlich (Oregon State Univ. and NOAA, 2030 SE Marine Science Dr., Newport, OR 97365, USA, Sara.Heimlich @ oregonstate.edu), Sharon Nieukirk (Oregon State Univ. and NOAA, 2030 SE Marine Science Dr., Newport, OR 97365, USA, Sharon.Nieukirk@oregonstate.edu)
\end{abstract}

A free archive has been developed for research in automatic detection/classification of cetacean sounds. The archive contains many datasets, with each dataset comprising recordings and metadata for a given species and geographic area. This archive differs from other sound archives in three important respects: (1) Recordings are annotated to indicate where in time and frequency the sounds of a given species occur. These annotations are done manually to remove the bias of any automatic detection system. (2) The archive deliberately includes poor-quality recordings, recordings encountered in any realistic detection/classification application. (3) Since performance of detection/classification methods depends heavily on the SNR of target sounds, the archive includes each vocalization's SNR so performance can be effectively represented. Until recently, most recordings in the archive were of baleen whales [Mellinger and Clark, Applied Acoustics 67(11), 2006]. However, datasets for toothed whales and dolphins are now being incorporated. Initial efforts have been focused on beaked whales because of high interest for management and because of the difficulty of visual detection. To date, recordings of Cuvier's, Blainville's, Baird's, and Arnoux's beaked whales have been entered into the archive, as have those of bottlenose whales, pilot whales, and some delphinid species. Further contributions are welcomed.

\section{Contributed Papers}

\section{0:40}

3aABb2. Dynamic tracking of free-swimming whale groups using digital acoustic recording tags. Charles White (University of Rhode Island, Department of Ocean Engineering, Narragansett Bay Campus, Narragansett, RI 02882, USA, cwhite@oce.uri.edu), James H. Miller (University of Rhode Island, Department of Ocean Engineering, Narragansett Bay Campus, Narragansett, RI 02882, USA, miller@egr.uri .edu), Gopu R. Potty (University of Rhode Island, Department of Ocean Engineering, Narragansett Bay Campus, Narragansett, RI 02882, USA, gopu@uri.edu), Mark Johnson (Woods Hole Oceanographic Institution, Applied Ocean Physics \& Engineering Dept., Woods Hole, MA 02543, USA, majohnson@whoi.edu)

Understanding the group behavior of free-swimming whales is critical to the management and conservation of many whale species. Moreover, understanding how whales coordinate their activities could serve as a model for improving the communication and navigation systems of underwater robots and autonomous underwater vehicles (AUVs). One method for studying the group behavior of free-swimming whales involves simultaneously attaching acoustic recording tags to multiple animals within a close-swimming conspecific group. Acoustic recording tags can sample an animal's acoustic environment simultaneously with other physical parameters such as diving depth and acceleration. In this paper, methods are presented for dynamically tracking whales that vocalize regularly such as odontocetes using the DTAG, a digital acoustic recording tag developed by the Woods Hole Oceanographic Institution. A trilateration model is reviewed for tracking groups of whales that include three or more tagged whales using time of flight data and results are given from applying this model to a novel data set of three simultaneously diving and cross-audible short-finned pilot whales
(Globicephala macrorhyncus). A new model is subsequently proposed for tracking whale groups that include only two tagged whales using a generalization of multilateration and data captured by stereo DTAGs.

\section{1:00}

3aABb3. Spatial confirmation of vocal communication between a killer whale calf and its natal family. Scott Veirs (Beam Reach Marine Science and Sustainability School, 7044 17th Ave NE, Seattle, WA 98115, USA, scott@beamreach.org), Val Veirs (Colorado College, Department of Physics, Colorado Springs, CO 80903, USA, vveirs@coloradocollege .edu), Jason D. Wood (Beam Reach Marine Science and Sustainability School, 7044 17th Ave NE, Seattle, WA 98115, USA, jason@beamreach .org)

We recorded an interchange of vocal signaling between a four-year-old Southern Resident killer whale and its natal family members (mother and brother). This information was recorded on a 30-m-long linear array of hydrophones that was towed behind our electric motor powered research vessel. Over the course of about $1 / 2$ hour we recorded signals from these isolated orcas about $500 \mathrm{~m}$ offshore of San Juan Island, WA. Surface locations of the orcas were documented with visual accounts, photographs, video, and - during the latter portion of the encounter - with a hand-bearing compass. During a six minute interval of the recording we have localized $\sim 70$ vocalizations that we can associate with the surface positions of either the juvenile or the mother-brother pair (they were close together). The central three minutes of this interval document an event in which the juvenile left the family group, swam toward our vessel, approached it within about $10 \mathrm{~m}$, turned abruptly away during a rapid call-response sequence with the 
mother and/or brother, and then rejoined the natal group. This rare event informs killer whale vocal development, repertoire, communication, and active space.

\section{1:20}

3aABb4. Killer whales (Orcinus orca) and Bell Laboratories: What can information theory tell us about the communication system of this species? Kenna D. Lehmann (University of California, Dept. of Evolution and Ecology, Davis, CA 95616, USA, kdlehmann@ucdavis.edu), Jason D. Wood (Beam Reach Marine Science and Sustainability School, 7044 17th Ave NE, Seattle, WA 98115, USA, jason@ beamreach.org)

In the 1950's Shannon and Weaver developed information theory as a statistical tool to determine the structure and organization within communication systems. More recently information theory has been applied to the study of animal communication. We gathered acoustic recordings of the Southern Resident killer whales in the inland waters of Washington State, USA, and British Columbia, Canada, from both land based and boat based acoustic arrays. Recorded calls were categorized using the accepted call catalog for this population. In this paper we will present our results from applying various Shannon entropies to the documented call sequences. One notable result is that the call repertoire is more redundant than is ideal to convey the maximum amount of information. This may be a result of their particular social system and behavioral ecology, or may be an indication of the amount of noise present in their acoustic environment. Comparisons will also be made with measures of information theory in other species.

\section{1:40}

3aABb5. Whistle repertoire of captive bottlenose dolphins, Tursiops truncatus. Rebeca M. Lopez-Rivas (UNAM, Facultad de Ciencias, Depto. Física, Lab Acústica, Circuito exterior s/n, Cd. Universitaria, 04510 México, D.F., Mexico, rmlopez.rivas@gmail.com), Volker B. Deecke (University of British Columbia, Marine Mammal Research Unit, Vancouver, BC V6T 1Z4, Canada, deecke@zoology.ubc.ca), Carmen Bazúa Durán (UNAM, Facultad de Ciencias, Depto. Física, Lab Acústica, Circuito exterior s/n, Cd. Universitaria, 04510 México, D.F., Mexico, bazua@servidor.unam.mx)

The whistle repertoire of bottlenose dolphins has been poorly described in the literature, thus little information is known to support the hypothesis that whistles are used by dolphins to communicate. In this study, whistles emitted by four captive bottlenose dolphins housed in two aquaria in Mexico City were recorded to analyze the whistle repertoire and whistle emission rate for each aquarium. The whistle repertoire was described using human and automated categorizations. Results show that whistle emission rate and the whistle repertoire are both larger for the two dolphins that are more active and which are housed in the same aquarium. As for whistles shared in the repertoire of both aquaria, these are very few, probably because dolphins of the two aquaria were captured in different oceans and are of different ages, besides of the different activities that dolphins can perform in each aquarium. Additionally, all four dolphins could use similar whistles for a specific activity, like "swimming", while different whistles were used for another specific activity, like "observing". These results show that dolphins use whistles selectively, supporting the hypothesis that whistles may be used to communicate information for particular situations. [Work supported by PAPIIT, UNAM \& CoNaCyT]

\section{2:00}

3aABb6. Unsupervised clustering of whistle-sounds of bottlenose dolphins (Tursiops truncatus). Sebastian V. Huebner (Pestalozzistrasse 5, 14482 Potsdam, Germany, sebastian@ sejona.de)

An unsupervised symbolic clustering algorithm for whistles of bottlenose dolphins (Tursiops truncatus) was tuned on an artificial data set containing 12 ideal synthetic signature whistles. Afterwards approximately 25,000 representations of natural whistles were clustered with the tuned algorithm. Recordings were made from a group of twelve adult semi-free living animals in Eilat (Israel). Obtained clusters were evaluated with the help of their individual compression rates. The clustering algorithm perfectly converged on all twelve artificial whistle types. Also, automatic whistle extraction produced good representations of both artificial and natural whistles, but clustering of natural whistles provided low support for the signature whistle hypothesis: The six most frequent induced whistle types could be interpreted as slight variations of only two signature whistles. Also, a great variety of different but rare types in automatically generated clusters could be observed. Results suggest that certain aspects of the group behavior are manifest in the frequency whistle types occur.

\section{2:20}

3aABb7. Acoustic studies of dolphins in their natural habitat: Challenges and successes. Katherine Kim (HLS Research, Inc., 3366 N. Torrey Pines Ct., Ste. 310, La Jolla, CA 92037, USA, Katherine.Kim @ HLSResearch.com), Paul Hursky (HLS Research, Inc., 3366 N. Torrey Pines Ct., Ste. 310, La Jolla, CA 92037, USA, paul.hursky@hlsresearch .com), Michael B. Porter (HLS Research, Inc., 3366 N. Torrey Pines Ct., Ste. 310, La Jolla, CA 92037, USA, michael.porter@hlsresearch.com), John A. Hildebrand (Scripps Institution of Oceanography- UCSD, 9500 Gilman Dr. \#0205, La Jolla, CA 92093, USA, jhildebrand@ucsd.edu), Elizabeth Henderson (Scripps Institution of Oceanography- UCSD, 9500 Gilman Dr. \#0205, La Jolla, CA 92093, USA, e0hender@ucsd.edu), John Calambokidis (Cascadia Research Collective, 218 1/2 W. 4th Ave., Olympia, WA 98501, USA, calambokidis@ cascadiaresearch.org), Erin Falcone (Cascadia Research Collective, 218 1/2 W. 4th Ave., Olympia, WA 98501, USA, EFalcone@ cascadiaresearch.org)

Acoustic methods for studying marine mammals is a research area of great potential, with applications which include population estimation, spatial distribution, behavioral studies, and the effects of anthropogenic noise on animals. Until recently, much of the acoustic research on free-ranging animals has focused on mysticetes and sperm whales; however, concern over highly-publicized dolphin strandings has raised interest in other odontocetes. From acoustic and signal processing perspectives, dolphins pose numerous new challenges: They typically aggregate in pods composed of tens or hundreds of individuals, often vocalizing simultaneously, and their calls are highly variable and high-frequency. Detection algorithms will be presented which are robust (or, conversely, can be fine-tuned) to these variable calls and have been automated to overcome the ordinarily laborintensive analysis requirements of such large data sets. Historically, acoustic studies of marine mammals used relatively simple sensor systems comprised of one or few hydrophones. In our studies, multiple sensor systems were deployed. Doing so enabled the utilization of array and co-array processing techniques for improved localization estimation which, like our detection algorithms, were fully automated. They also raised important deployment considerations due to the acoustic propagation physics as well as challenges in call association between different systems.

\section{2:40}

3aABb8. Narrow band high frequency clicks from Hourglass dolphins (Lagenorhynchus cruciger). Line A. Kyhn (National Environmental Research Institute, University of Aarhus, Frederiksborgvej399, DK-4000 Roskilde, Denmark, 1ky@dmu.dk), Jakob Tougaard (National Environmental Research Institute, University of Aarhus, Frederiksborgvej399, DK-4000 Roskilde, Denmark, jat@dmu.dk), Magnus Wahlberg (Fjord\&Bælt / University of Southern Denmark, Margrethes Plads 1, 5300 Kerteminde, Denmark, magnus@fjord-baelt.dk), Kristian Beedholm (Institute of Biology, University of Southern Denmark, Campusvej 55, 5230 Odense M, Denmark, beedholm@mail.dk), Peter T. Madsen (University of Aarhus, Biological Sciences, Zoophysiology, C. F. Møllers Allé, Building 1131, DK-8000 Aarhus, Denmark, peter.madsen@biology.au.dk)

The source properties of toothed whale echolocation clicks are essential for evaluating the performance of biosonar systems. However, such data only exist for a small number of free ranging toothed whale species. Here we present a quantitative analysis of the first recordings of echolocation sounds from hour glass dolphins (Lagenorhynchus cruciger) made in the Drake Strait. Recordings were made with a vertical array of four broadband, calibrated hydrophones that allows for acoustic localization and source parameter derivation. Only apparent on-axis clicks were used for analysis. Hour glass dolphins produce clicks with a mean peak and centroid frequency around $128 \mathrm{kHz}$, a narrow, mean RMS bandwidth of $10 \mathrm{kHz}$ with no energy below $100 \mathrm{kHz}$ and a mean signal duration of $161 \mu \mathrm{s}$. Echolocation clicks of hour glass dolphins are thus of the high frequency narrow band- 
width type (NBHF type) found in porpoises, a few delphinids of the Cephalorhynchus genus and the pygmy sperm whale Kogia breviceps, but are strikingly different from other dolphins. Acoustic convergence from vastly different genera and ecotypes suggest that evolution of NBHF clicks are adaptations to special auditory processing or acoustic crypsis rather than to habitat.

WEDNESDAY MORNING, 2 JULY 2008

ROOM 342A, 8:00 TO 11:20 A.M.

\title{
Session 3aAO
}

\section{Acoustical Oceanography, Underwater Acoustics, Signal Processing in Acoustics, and ECUA: Geoacoustic Characterization of the Ocean Bottom and Geoacoustic Inversion III}

\author{
Peter Gerstoft, Cochair \\ Marine Physical Laboratory, Scripps Institute of Oceanography, 8602 La Jolla Shores Drive, La Jolla, CA 92093-0238, USA \\ Dag Tollefsen, Cochair \\ Norwegian Defence Research Establishment (FFI), Box 115, Horten, 3191, Norway
}

\section{Invited Papers}

8:00

3aAO1. Location of Targets and Scatterers with Ambient Noise. Christopher H. Harrison (NATO Undersea Research Centre, Viale San Bartolomeo 400, 19138 La Spezia, Italy, harrison@nurc.nato.int)

By placing a vertical array in an ambient noise field and forming an upward and downward beam one obtains two time series which can be cross-correlated to reveal a sub-bottom profile of the seabed [Siderius, et al., J. Acoust. Soc. Am., 120, 1315-1323, (2006)]. Here the cross-correlation approach is extended to the location in range and bearing of a point target. An experiment was designed using floats and weights mounted (and dismounted) on the same cable as the vertical array. Careful measurements were made of the location of all likely floats, ballast weights, array terminations, and so on. After suitable coherent averaging, peaks were seen at delays (correlation offsets) agreeing with the reflector positions and were shown to be absent when reflectors were removed. An implication for geoacoustic inversion is that, in priciple, the technique can be used to explore scattering as well as reflection properties of layers. A simple extension of the theory developed in [Harrison and Siderius, J. Acoust. Soc. Am., (2007), accepted] is used to explain the amplitudes of the reflections. The approach differs from "acoustic daylight" principally in determining a target range.

8:20

3aAO2. Wide-area geoacoustic inversion using distant ship noise. Altan Turgut (Naval Research Laboratory, Acoustics Division, Code 7120, Washington, DC 20375, USA, altan.turgut@nrl.navy.mil)

Broadband $(50-500 \mathrm{~Hz})$ noise data from fast-traveling surface ships in the vicinity of a VLA and a bottomed HLA are used to estimate the geoacoustic properties on the New Jersey Shelf. Matched-field inversion of geometric and geoacoustic parameters are presented by using twenty days of ship noise data, collected during the RAGS03 winter experiment. Noise data from different ships at different ranges provided robust geoacoustic inversions for both VLA and bottomed HLA data. Acoustic propagation tracks are determined from the HLA beamforming results and a 3-D geoacoustic parameter map is constructed. The constructed geoacoustic parameter map shows good agreement with the results from chirp sonar inversion and acoustic probe measurements, indicating silty seafloor sediments on the outer-shelf area and sandy sediments on the inner-shelf area. Efficiency of the 3-D geoacoustic inversion and extension to range-dependent inversions at longer ranges are also discussed. [Work supported by ONR.]

\section{8:40}

3aA03. Doppler Geo-Spectroscopy in the Makai Experiment. David R. Barclay (Marine Physical Lab, Scripps Institution of Oceanography, UCSD, 9500 Gilman Dr. M/C 0238, La Jolla, CA 92093-0238, USA, dbarclay@ mpl.ucsd.edu), Michael Buckingham (Marine Physical Lab, Scripps Institution of Oceanography, UCSD, 9500 Gilman Dr. M/C 0238, La Jolla, CA 92093-0238, USA, mjb @ mpl.ucsd.edu)

Using data collected during the 2005 Makai experiment, acoustic parameters of a coral-sand seabed were obtained using the Doppler spectroscopy inversion technique. A light aircraft was used as a low frequency $(80-400 \mathrm{~Hz})$ sound source over an isovelocity shallow water channel. A microphone near the ocean surface and a hydrophone near the seabed were used to record the Doppler spreading of each harmonic of the aircraft's engine and propeller. In the water column, spectral peaks with the greatest up and down Doppler shifts are associated with the shallow equivalent rays of normal modes. The least mean squares (LMS) adaptive filter was used to track and enhance the frequency of these propagating modes. The inversion relies on a full wave number spectral model of a moving source in 
a three-layer waveguide. The Doppler shifted modes in the water column are observed in the data and simulated in the model. The comparison of the two, in symphony with the aircraft's signal in the air, returns the geoacoustic parameters of the seabed. The inversion was performed on numerous over-flights and the results were averaged to show statistical confidence. Work supported by ONR.

\section{Contributed Papers}

\section{9:00}

3aAO4. Geoacoustic inversion in the frequency range $0.8-1.6 \mathrm{kHz}$ with drifting sparse arrays during MREA/BP'07 experiment. Jean-Claude Le Gac (NATO Undersea Research Center, Viale San Bartolomeo 400, 19126 La Spezia, Italy, legac@nurc.nato.int), Jean-Pierre Hermand (Université libre de Bruxelles (U.L.B.) - Environmental hydroacoustics lab, av. Franklin D. Roosevelt 50, CP 194/5, 1050 Bruxelles, Belgium, jhermand@ulb.ac.be), Sergio Jesus (ISR, Universidade do Algarve, PT-8005-139 Faro, Portugal, sjesus@ualg.pt)

In order to evaluate properly the acoustic propagation characteristics in shallow water environments, it is well established that appropriate knowledge of the acoustic properties of the seabottom is required. In the last decade, full-field geoacoustic inversion techniques have been demonstrated to provide adequate methodologies to assess those properties. However, several of the developed techniques may suffer a lack of adequacy to the design of low-frequency active sonar systems (LFAS) for which the assessment of seabottom characteristics are drawn. For instance most matched-field inversion techniques demonstrated so far use acoustical signals at much lower frequencies than those of the sonar. Furthermore, some of the techniques may be difficult to be handled in an "operationally relevant context" since they are based on relatively complex designed systems such as highly instrumented vertical line arrays spanning the whole water column. In this paper, we investigate the potential of medium-frequency acoustical signals $(0$ $.8-1.6 \mathrm{kHz}$ ) received at several ranges on a field of drifting sparse arrays, eventually reduced to a couple of hydrophones, for spatially-coherent geoacoustic inversion purposes. The experimental datasets of the Maritime Rapid Environmental Assessment MREA/BP'07 sea trial south of Elba Island in the Mediterranean Sea are used to support this study.

\section{9:20}

3aA05. Using offshore seismic surveys as acoustic sources of opportunity for geoacoustic inversion. Alec J. Duncan (Curtin University of Technology, Centre for Marine Sci \& Tech, GPO Box U1987, 6845 Perth, WA, Australia, A.J.Duncan@curtin.edu.au), Alexander Gavrilov (Curtin University of Technology, Centre for Marine Sci \& Tech, GPO Box U1987, 6845 Perth, WA, Australia, a.gavrilov@cmst.curtin .edu.au), Tao Hu (Institute of Acoustics, Chinese Academy of Science, PostBox 2712, NO.21, Bei-Si-huan-Xi Road, 100080 Beijing, China, hutao76@yahoo.com.cn)

Commercial offshore seismic surveys involve the use of powerful acoustic sources consisting of arrays of airguns. These sources produce frequent, loud, impulsive sounds at precisely timed intervals, at accurately known positions during surveys that may last for several months. By deploying a lowcost acoustic receiving system in the vicinity of such a survey scientists can acquire high quality data for geoacoustic inversion experiments in an extremely cost-effective manner. This paper discusses the various factors that must be considered when using such data for geoacoustic inversion, including the prediction of source spectra and array directionality, and provides some examples.

\section{9:40-10:00 Break}

\section{0:00}

3aA06. Geo-acoustic inversion with adaptive beamformed data. Steven A. Stotts (Applied Research Laboratories/The University of Texas, 10000 Burnet Rd, Austin, TX 78758, USA, stotts@arlut.utexas.edu), Robert A. Koch (Applied Research Laboratories/The University of Texas, 10000 Burnet Rd, Austin, TX 78758, USA, koch@arlut.utexas.edu)

Geo-acoustic inversion with matched field replicas constructed by adaptive techniques lacks robustness, because localizations with such techniques are sensitive to model mismatch [Soares, Jesus, and Coelho, J. Acoust. Soc. Am. 122, 3391-3404 (2007)]. Instead of adapting the matched field weights, inversion with adaptive beam-formed (ABF) plane wave sub-aperture data produces accurate source localizations for ships of opportunity [Stotts and Hawkins, J. Acoust. Soc. Am. 118, 1857 (2005)]. Model inputs to the matched-field cost function are evaluated at sub-aperture phase centers in this approach. The main purpose of this talk is to demonstrate the robustness and accuracy of solutions obtained from geo-acoustic inversion using plane wave ABF data. Improvements over either single element or conventional plane wave beam-formed (CBF) sub-aperture data for geo-acoustic inversions and simultaneous geo-acoustic inversions/source localizations using a source of opportunity in real data containing interferers will be demonstrated.

\section{0:20}

3aA07. Geoacoustic inversion with recreational boat noise. A. Vincent Van Leijen (Netherlands Defence Academy, P.O. Box 10 000, 1780 CA Den Helder, Netherlands, av.v.leijen@nlda.nl)

During the MREA07 sea trials various experiments involved geoacoustic inversion with sound sources of opportunity. A particular experiment that focussed on exploiting the self noise of autonomous vehicles suffered from interfering noise due to increased boating activity in the weekend. In this work the same data is re-examined to study the potential of recreational boats as sound sources for geoacoustic inversion. Even though recreational boats are non cooperative sound sources, in the sense that weekend traffic does not sail predefined tracks and navigational logging is absent, various boats swarm out and cover large areas thus acting as a distributed set of sound sources. A typical small boat signature counts numerous harmonics of narrowband tones that cover the frequency spectre from $100 \mathrm{~Hz}$ up to $2 \mathrm{kHz}$. With these tones matched field inversion and traditional Doppler techniques are used to find an initial geometry of the experiments and these results are fine tuned during the final geoacoustic inversion of a locally rangeindependent model of a coastal environment. Results of inversions with recreational boating sounds are shown to match with previous analysis results with self noise of autonomous vehicles, bottom grab samples and seismic data.

\section{0:40}

3aAO8. Geoacoustic uncertainties in matched-field inversions of ship-noise data. Dag Tollefsen (Norwegian Defence Research Establishment (FFI), Box 115, 3191 Horten, Norway, dag.tollefsen@ffi.no), Stan E. Dosso (University of Victoria, School of Earth and Ocean Sciences, Victoria, BC V8W 3P6, Canada, sdosso@uvic.ca)

This paper applies geoacoustic inversion to ship-noise data recorded on a horizontal array deployed on the seafloor in an experiment conducted by FFI in the Barents Sea. A Bayesian matched-field inversion method is employed which quantifies geoacoustic uncertainties and allows for quantitative comparisons of results from different data sets. Estimates of sediment parameters and their uncertainties were obtained by inversions of lowfrequency narrowband data from a quiet research ship at source-array ranges of 1-6 km and different orientations with respect to the array, as well as from a merchant ship at ranges of $6-8 \mathrm{~km}$. Multiple, independent data segments are included in the inversion and shown to significantly reduce geoacoustic parameter uncertainties. The effect of the state of prior information on the ship track (e.g., well known or unknown) on geoacoustic information content is also examined. Estimates of the sound-speed profile and density of sediment are compared with results from inversion of data from a towed controlled-source, and with reference values from other geophysical data collected in the area. 
11:00

3aA09. Optimal source tracking in an unknown ocean environment. Michael J. Wilmut (University of Victoria, School of Earth and Ocean Sciences, Victoria, BC V8W 3P6, Canada, mjwilmut@uvic.ca), Stan E. Dosso (University of Victoria, School of Earth and Ocean Sciences, Victoria, BC V8W 3P6, Canada, sdosso@uvic.ca)

This paper develops and compares two approaches to localizing and tracking a low-level acoustic source when ocean environmental properties are unknown. Both approaches are based on a Bayesian formulation in which source and environmental parameters are considered random variables constrained by noisy acoustic data and by prior information on parameter values (e.g., physical limits for water-column and seabed properties) and on inter-parameter relationships (e.g., limits on horizontal and vertical source speed). One approach is based on determining the source track and environmental parameters that maximize the posterior probability density (PPD) using adaptive hybrid optimization. A key to solving this challenging problem efficiently is that the Viterbi algorithm is applied to compute the highest-probability source track for each environmental realization considered in the optimization-this provides the optimal track while requiring the optimization is applied only to environmental parameters. The second approach involves integrating the PPD over unknown environmental parameters to represent source-track information as a series of joint marginal probability surfaces over range and depth. The Viterbi algorithm is applied to extract the optimal track from these surfaces. For realistic environmental models (e.g., more than a dozen unknown parameters), the integration is carried out using efficient Markov-chain Monte Carlo methods.

WEDNESDAY MORNING, 2 JULY 2008

ROOM 352B, 8:00 TO 10:40 A.M.

\title{
Session 3aBBa
}

\section{Biomedical Ultrasound/Bioresponse to Vibration and Physical Acoustics: Shock Waves in Medicine}

\author{
Robin O. Cleveland, Cochair \\ Boston University, Dept. of Aerosp. and Mech. Eng., 110 Cummington St., Boston, MA 02215, USA \\ Oleg A. Sapozhnikov, Cochair \\ Center for Industrial and Medical Ultrasound, Applied Physics Lab., University of Washington, 1013 NE 40th St., Seattle, WA \\ 98105, USA
}

Invited Paper

8:00

3aBBa1. Short- and long-delay tandem shock waves for application in medicine. Achim M. Loske (Universidad Nacional Autónoma de México, Centro de Física Aplicada y Tecnología Avanzada, AP 1-1010, 76000 Querétaro, Mexico, loske@fata .unam.mx), Francisco Fernandez (Universidad Nacional Autónoma de México, Centro de Física Aplicada y Tecnología Avanzada, AP 1-1010, 76000 Querétaro, Mexico, francisco@fata.unam.mx), Miguel De Icaza (Universidad Nacional Autónoma de México, Centro de Física Aplicada y Tecnología Avanzada, AP 1-1010, 76000 Querétaro, Mexico, icaza@ fata.unam.mx), Guillermo Canseco (Universidad Nacional Autónoma de México, Centro de Física Aplicada y Tecnología Avanzada, AP 1-1010, 76000 Querétaro, Mexico, gcanseco@fata.unam.mx)

Long-delay tandem shock waves with an inter-pulse delay between about 200 and 500 microseconds have shown to enhance fragmentation of artificial kidney stones during in vitro and in vivo extracorporeal shock wave lithotripsy (SWL) in the past. Cellpermeabilization and inactivation of bacteria using tandem shock waves have also been proposed. The purpose of this research was to study the influence of short-delay (less than 50 microseconds) tandem shock waves on cavitation induced damage to kidney stones during SWL. A multipurpose piezoelectric shock wave generator was designed to generate shock waves at variable delays. The effect of short- and long-delay dual-pulse shock waves on the surface of artificial kidney stones was evaluated by microscopy. An adequate selection of delays was possible by means of a numerical model and high speed photography. Stone fragmentation was compared to that of standard (single-pulse) shock waves. Our results indicate that specific combinations of short- and long-delay tandem shock waves could improve damage to kidney stones by acoustic cavitation. 


\section{Contributed Papers}

\section{8:20}

3aBBa2. Correlation of vasoconstriction and kidney protection during shock wave lithotripsy. Michael R. Bailey (Center for Industrial and Medical Ultrasound, Applied Physics Lab., University of Washington, 1013 NE 40th St., Seattle, WA 98105, USA, bailey@ apl.washington.edu), Marla Paun (Center for Industrial and Medical Ultrasound, Applied Physics Lab., University of Washington, 1013 NE 40th St., Seattle, WA 98105, USA, paun@u.washington.edu), Rajash K. Handa (Department of Anatomy and Cell Biology, Indiana University School of Medicine, 635 Barnhill Dr. MS5055, Indianapolis, IN 46202, USA, rhanda@iupui.edu), Sujuan Gao (Department of Medicine, Division of Biostatistics, Indiana University School of Medicine, 410 West 10th Street, Suite 3000, Indianapolis, IN 46202, USA, sgao@iupui.edu), Lynn R. Willis (Department of Pharmacology, Indiana University School of Medicine, 635 Barnhill Dr. MS5055, Indianapolis, IN 46202, USA, willisl@iupui.edu), Bret A. Connors (Department of Anatomy and Cell Biology, Indiana University School of Medicine, 635 Barnhill Dr. MS5055, Indianapolis, IN 46202, USA, connors@anatomy.iupui.edu), James A. McAteer (Department of Anatomy and Cell Biology, Indiana University School of Medicine, 635 Barnhill Dr. MS5055, Indianapolis, IN 46202, USA, mcateer @ anatomy.iupui.edu), Andrew P. Evan (Department of Anatomy and Cell Biology, Indiana University School of Medicine, 635 Barnhill Dr. MS5055, Indianapolis, IN 46202, USA, aevan@iupui.edu)

Kidney injury in lithotripsy can lead to long-term adverse effects, so minimizing injury is beneficial. We have found that injury is significantly reduced when shock wave (SW) administration is halted briefly-3-minutesearly in treatment. Previous studies have shown that SWs stimulate renal blood vessels to constrict. Therefore, we tested the idea that vasoconstriction mediates SW-induced protection of the kidney. Doppler ultrasound was used to measure resistive index (RI), a ratio of systolic and diastolic velocities, indicating vasoconstriction. RI was determined for single intralobar arteries in targeted porcine kidneys treated by standard versus protection protocols $(2,000 \mathrm{SW}$, uninterrupted vs $500 \mathrm{SW}-3$-min pause-2,000SW) using a DornierHM3 lithotripter $(2 \mathrm{~Hz}, 24 \mathrm{kV})$. Significant differences in RI from baseline within a group and between groups at various time points were determined using mixed-effect models for repeated measures with Holm's step-down method for multiple comparison adjustment. All animals had similar baselines. Sham pigs (no-SWs) showed no significant change in RI. The protection protocol produced a significant rise $(\mathrm{p}<0.05, \mathrm{n}=8)$ in RI 15 minutes into treatment, while the standard protocol did not yield a significant rise $(\mathrm{p}<0.05, \mathrm{n}=7)$ until 45 minutes after treatment. Thus, the treatment protocol shown to protect against injury, induces early vasoconstriction. [NIH-DK43881, NSBRI-SMS00402]

\section{8:40}

3aBBa3. Fundamental study of shock induced acceleration of cel growth by plane shock waves. Masaaki Tamagawa (Kyushu Institute of Technology, Hibikino 2-4, Wakamatsu-ku, 808-0196 Kitakyushu, Japan, tama@life.kyutech.ac.jp)

This paper describes effects of shock waves on cells to certificate the angiogenesis by shock wave in the clinical application. Especially, the effects of plane shock waves without cavitation bubbles on the endothelial cells are investigated by experiments and computation. In the experiments, the plane shock waves using shock tube apparatus are applied to the endothelial cells. The peak pressure working on the cells in the test case is 0.4 $\mathrm{MPa}$, which is under disintegration level for endothelial cells. After working shock waves on suspended cell, the disintegration and growth rate are measured. It is found that the initial growth rate of the shock-worked cells are clearly higher than that of control ones. As for the effect of rise time of working shock waves, to change the rise time of shock wave, Air and He gas are used as driver gas in the shock tube. As a result, the shorter rise time brings higher growth rate. In the computation, to check the effects of rise time on the pressure and stress distribution on the cells, fluid and structural interaction model are analyzed. The result shows that shorter rise time makes high stress level distribution on the cell membrane.

\section{9:00}

3aBBa4. Beamwidth measurement of single lithotripter shock waves. Wayne Kreider (Center for Industrial and Medical Ultrasound, Applied Physics Lab., University of Washington, 1013 NE 40th St., Seattle, WA 98105, USA,wkreider@u.washington.edu), Michael R. Bailey (Center for Industrial and Medical Ultrasound, Applied Physics Lab., University of Washington, 1013 NE 40th St., Seattle, WA 98105, USA, bailey@apl .washington.edu), Jeffrey A. Ketterling (Frederic L. Lizzi Center for Biomedical Engineering, Riverside Research Institute, 156 William St., New York, NY 10038, USA, ketterling@ rrinyc.org)

New lithotripters with narrower foci and higher peak pressures than the original Dornier HM3 electrohydraulic lithotripter have proven to be less effective and less safe. Hence, accurate measurements of the focal characteristics of lithotripter shock waves are important. The current technique for measuring beamwidth requires collection of single-point measurements over multiple shock waves, thereby introducing error as a result of any shock-toshock variability. This work reports on the construction of a hydrophone array sensor and on array measurements of individual lithotripter shock waves. Beamwidths for an electrohydraulic lithotripter with a broad-focus, HM3-style reflector and a narrow-focus, modified reflector were measured using both new and worn electrodes as well as two different electrical charging potentials. The array measured the waveform, beamwidth, and focal location of individual shock waves. The HM3-style reflector produced repeatable focal waveforms and beam profiles at an $18-\mathrm{kV}$ charging potential with both new and worn electrodes. Accordingly, these measurements agreed with averaged point measurements acquired under the same conditions. However, a lack of consistency in the measured focal locations and beam profiles at $23 \mathrm{kV}$ underscores the value in obtaining beam profile measurements from individual shock waves. Work supported by Riverside Research Institute, NIH DK43881, and NSBRI SMS00402.

\section{9:20}

3aBBa5. Numerical simulation of shock wave generation and focusing in shock wave lithotripsy. Jeffrey Krimmel (California Institute of Technology, 1200 E. California Blvd, Pasadena, CA 91125, USA, jkrimmel @ caltech.edu), Tim Colonius (California Institute of Technology, 1200 E. California Blvd, Pasadena, CA 91125, USA, colonius@ caltech.edu)

Shock wave lithotripsy is a procedure where focused shock waves are fired at kidney stones in order to pulverize them. Lithotripters with different source mechanisms and reflector shapes (or lenses) are in clinical use, but prediction of focal region pressure is made difficult by nonlinearity and cavitation. We report on development of a numerical simulation framework aimed at accurate prediction of focal region pressures and bubble dynamics. Shock wave generation and beam focusing are simulated via the Euler equations with MUSCL-type shock-capturing scheme and adaptive mesh refinement (AMR). Electrohydraulic, electromagnetic, and piezoelectric-array lithotripters are modeled with axisymmetric geometries. In the electrohydraulic case, an expanding bubble model simulates spark firing. In the piezoelectric case, a boundary condition prescribing the motion of individual elements is used. Calculated peak pressures and pulse widths agree well with experimental data for the electrohydraulic and electromagnetic lithotripters. For the piezoelectric case, peak pressures are increasingly overpredicted by the model when more elements are fired, and reasons for this discrepancy will be discussed. Finally, we compare the spatial and temporal characteristics of the focal pressure fields for several clinical lithotripters in the context of observed stone comminution efficacy and tissue damage. This work is supported by NIH grant PO1 DK043881 and ONR Grant N0001406-1-0730.

9:40

3aBBa6. Effect of elastic waves in the metal reflector on bubble dynamics at the focus of an electrohydraulic lithotripter. Oleg A. Sapozhnikov (Center for Industrial and Medical Ultrasound, Applied Physics Lab., University of Washington, 1013 NE 40th St., Seattle, WA 98105, 
USA, oleg@acs366.phys.msu.ru),Wayne Kreider(Center for Industrial and Medical Ultrasound, Applied Physics Lab., University of Washington, 1013 NE 40th St., Seattle, WA 98105, USA, wkreider@u.washington .edu), Michael R. Bailey (Center for Industrial and Medical Ultrasound, Applied Physics Lab., University of Washington, 1013 NE 40th St., Seattle, WA 98105, USA, bailey@apl.washington.edu), Vera A. Khokhlova (Center for Industrial and Medical Ultrasound, Applied Physics Lab., University of Washington, 1013 NE 40th St., Seattle, WA 98105, USA, vera@acs366 .phys.msu.ru), Francesco Curra (Center for Industrial and Medical Ultrasound, Applied Physics Lab., University of Washington, 1013 NE 40th St., Seattle, WA 98105, USA, fcurra@apl.washington.edu)

In extracorporeal electrohydraulic lithotripters, a hemi-ellipsoidal metal reflector is used to focus a spherical wave generated by an electrical discharge. The spark source is positioned at one of the ellipsoid foci (F1); this makes the reflected wave focused at the other focus (F2). Despite the common assumption that the reflector behaves as a rigid mirror, the true reflection phenomenon includes the generation and reverberation of elastic waves in the reflector, which reradiate to the medium. Although these waves are much lower in amplitude than the specularly reflected wave, they may influence cavitation at F2. To explore such effects, waves in water and a brass reflector were modeled in finite differences based on the linearized equations of elasticity. The bubble response was simulated based on a Rayleigh-type equation for the bubble radius. In addition, the role of acoustic nonlinearity was estimated by numerical modeling. It is shown that the elastic waves in the reflector give rise to a long "ringing" tail, which results in nonmonotonic behavior of the bubble radius during its inertial growth after shock wave passage. This numerical result is qualitatively confirmed by experimental observations of bubble behavior using high-speed photography. Work supported by NIH-DK43881, NSBRI-SMS00402, NIHDK075090, and RFBR.

\section{0:00}

3aBBa7. Elastic waves in human kidney stones: Shear dominates spall in shock wave lithotripsy. Robin O. Cleveland (Boston University, Dept. of Aerosp. and Mech. Eng., 110 Cummington St., Boston, MA 02215, USA, robinc@bu.edu), Haibiao Luo (Boston University, Dept. of Aerosp. and Mech. Eng., 110 Cummington St., Boston, MA 02215, USA, hluo @bu.edu), James C. Williams (Indiana Univ. School of Medicine, 635 Barnhill Dr, Indianapolis, IN 46202, USA, williams@anatomy.iupui.edu)

We investigated the role of shear waves and spall in the fragmentation of human kidney stones. Seven human kidney stones, approximately $5 \mathrm{~mm}$ in diameter, were scanned with micro computed tomography to obtain a 3D representation that was imported into an elastic wave code. The code was initialized with a lithotripsy waveform and the evolution of stresses and strains was tracked through space and time. The evolution of the principal stress indicated that reflection from the distal surface of the stone (spallation) did not induce large tensile stresses $(<40 \mathrm{MPa})$ due to the curved geometry of the proximal and distal surfaces. Instead as the shock wave in the fluid passed the mid-section of the stone there was efficient coupling into shear waves which then propagated into the interior of the stone and generated the largest regions of high tensile stress ( $>60 \mathrm{MPa})$. The results indicate that for the human stones studied here shear waves generated at the stone periphery are more important than spall in generating high tensile stress. These data suggest that the focal width of a lithotripter needs to be larger than the stone in order to generate large stresses. [Work supported by NIH DK43881 and DK59933].

\section{0:20}

3aBBa8. Damage potential of single-bubble collapse in shockwave lithotripsy. Eric Johnsen (California Institute of Technology, $1200 \mathrm{E}$. California Blvd, Pasadena, CA 91125, USA, johnsen@stanford.edu), Tim Colonius (California Institute of Technology, 1200 E. California Blvd, Pasadena, CA 91125, USA, colonius@ caltech.edu)

In shockwave lithotripsy, the combined effect of focused shockwaves and cavitation pulverizes kidney stones. Although cavitation is known to play an important role in stone comminution, the underlying mechanism is not fully understood. The goal of the present study is to quantify the potential damage caused by Rayleigh collapse (RC) and shock-induced collapse (SIC) of a single bubble near a stone. A high-order accurate, quasiconservative, shock- and interface-capturing scheme [E. Johnsen and T. Colonius, J. Comput. Phys. 2006] is employed to simulate both phenomena. A high-speed re-entrant jet forms during the collapse and hits the distal side of the bubble, thereby generating a water-hammer pressure wave. A high pressure is measured along the stone surface upon the impact of this wave, thus providing potential for erosion. In SIC, this pressure may reach $1 \mathrm{GPa}$ and bubbles within approximately 15 initial radii generate a pressure higher than the incoming pulse. In addition, by using the present results as timedependent boundary conditions for an elastic wave propagation code, it is shown that a tension large enough to lead to failure may be achieved within small stones or fragments. This work is supported by NIH grant PO1 DK043881 and ONR Grant N00014-06-1-0730. 


\title{
Session 3aBBb
}

\section{Biomedical Ultrasound/Bioresponse to Vibration and Physical Acoustics: Light and Sound for Medical Imaging and Therapy I}

\author{
Ronald A. Roy, Cochair \\ Boston University, Dept. of Aerosp. and Mech. Eng., 110 Cummington St., Boston, MA 02215, USA \\ Emmanuel Bossy, Cochair \\ Laboratoire Photons et Matière, ESPCI/CNRS, 10 rue Vauquelin, Paris Cedex 05, 75231, France
}

\section{Invited Papers}

\section{1:00}

3aBBb1. Photoacoustic imaging for characterising the structure and function of vascular networks. Paul C. Beard (University College London, Gower Street, London, WC1E 6BT London, UK, pbeard@medphys.ucl.ac.uk)

Photoacoustic imaging is a new non invasive soft tissue imaging method in which low energy nanosecond pulses of visible or near infrared (NIR) laser light are used to excite subsurface ultrasound waves. The distinguishing advantage of the technique is that provides the high contrast and spectroscopic based specificity of optical techniques along with the high spatial resolution of ultrasound. Haemoglobin represents the most important source of naturally occurring photoacoustic contrast due its relatively strong optical absorption in the visible and NIR making the technique particularly well suited to imaging blood vessels. In addition, functional information can be provided by obtaining images at multiple wavelengths and applying a spectroscopic analysis to quantify the concentrations of endogenous chromophores such as oxy and deoxyhaemoglobin. These attributes make the technique well suited to studying a wide range of tissue abnormalities such as tumours and other pathologies characterised by changes in the structure and oxygenation status of the vasculature. A novel optical ultrasound imaging system has been developed and used to obtain 3D images of the superficial vascular anatomy of the mouse brain, skin and implanted tumours and demonstrate the spectroscopic capability of the technique.

\section{1:20}

3aBBb2. The role of ultrasound and photoacoustic imaging in laser therapy of cancer. Stanislav Y. Emelianov (Applied Research Laboratories, The University of Texas, P.O. Box 8029, Austin, TX 78713-8029, USA, emelian@mail.utexas.edu)

Laser therapy is an alternative cancer treatment approach when traditional surgery cannot be used to remove small, poorly defined lesions embedded within vital organs. For example, in photothermal laser therapy, a localized temperature increase to cause tumor necrosis is achieved by using a continuous wave laser and molecular targeted and optically tuned photoabsorbers. However, for laser therapy to be successful, it is necessary to identify the presence of photoabsorbers in the tumor prior to therapy, to monitor the spatiotemporal temperature changes during therapy, and, finally, to identify thermal/mechanical damage and tumor destruction after therapy. To guide laser therapy, we developed a combined ultrasound/photoacoustic imaging system and bioconjugated nanoabsorbers. In our approach, ultrasound imaging is utilized to identify the anatomy of the tumor while photoacoustic imaging is used to ensure the presence of targeted nanoparticles before therapy. Both ultrasound and photoacoustic imaging techniques are then used to monitor temperature during therapy, ensuring tumor necrosis and protection of the surrounding healthy tissue. Finally, ultrasound and elasticity imaging is used to confirm thermal damage and cancer destruction. The results of our studies indicate that photoacoustic and ultrasound imaging can be used to effectively guide laser therapy.

\section{1:40}

3aBBb3. Photoacoustic cancer imaging. Wiendelt Steenbergen (University of Twente /Institute for Biomedical Technology, PO Box 217, NL-7500 AE Enschede, Netherlands, w.steenbergen@utwente.nl)

Photoacoustics, or optoacoustics, involves ultrasound imaging based on ultrasound generated by absorption of light by tissue chromophores such as blood and melanin. Ultrasound is generated when pulsed or intensity modulated light is used which locally deposits a sufficient amount of energy in a sufficiently short time to cause an efficient generation of internal stress in the tissue. When this stress relaxes, an ultrasound wave is emitted which can be detected at the surface of the tissue. On the basis of these ultrasound waves, a threedimensional image the internal distribution of absorption can be constructed. We will discuss various technical implementations of the technique, and will illustrate various medical applications. A particularly important application field is oncology: in cancer, the presence of blood vessels is essential for the tumor to grow and spread. We will show results of photoacoustic imaging performed on small animals with implanted tumors. It appears, that measurement speed is a key issue in obtaining images with sufficient quality. Furthermore, we will show results obtained with our photoacoustic mammoscope on patients with breast cancer. 
3aBBb4. Theory and applications of frequency domain photoacoustic microscopy. Todd W. Murray (Boston University, 110 Cummington Street, Aerospace and Mechanical Engineering, Boston, MA 02215, USA, twmurray@ bu.edu), Suraj Bramhavar (Boston University, 110 Cummington Street, Aerospace and Mechanical Engineering, Boston, MA 02215, USA, suraj10@bu.edu), Ashwin Sampathkumar (Boston University, 110 Cummington Street, Aerospace and Mechanical Engineering, Boston, MA 02215, USA, suraj10@bu.edu), Bruno Pouet (Bossa Nova Technologies, 606 Venice Boulevard, Venice, CA 90291, USA, Bpouet@ BossaNovaTech .com)

In frequency domain photoacoustic microscopy, the pulsed laser source used for ultrasound excitation in conventional photoacoustic imaging is replaced by a low power, amplitude modulated laser source. The acoustic signals are detected using an interferometer or contact transducer, coupled to a RF lock-in amplifier or vector network analyzer. The detection bandwidth reduction afforded by this technique allows for a significant improvement in signal-to-noise ratio (SNR) over systems using pulsed laser excitation. In this paper, the method of frequency domain photoacoustic microscopy is reviewed and compared to the pulsed-laser based approach. Methods for processing the frequency domain data to extract the information of interest are discussed, along with the effects of measurement bandwidth and frequency resolution. A new technique to optically downshift acoustic signals detected using an optical interferometer to a fixed intermediate frequency is presented, which allows for the detection of high frequency (100's of MHz- GHz) acoustic signals using low frequency, low cost detection electronics. Several applications of frequency domain photoacoustic microscopy are presented including the inspection of thin films and environmental barrier coatings and the photothermal operation of nano-electro-mechanical systems. Finally, potential applications of this technique for the characterization of biological media are discussed.

\section{Contributed Papers}

\section{$12: 20$}

3aBBb5. Photoacoustic microscopy for high-resolution imaging. Wolfgang Bost (Fraunhofer IBMT, Ensheimer Strasse 48, 66386 Sankt Ingbert, Germany, wolfgang.bost@ibmt.fhg.de), Robert M. Lemor (Fraunhofer IBMT, Ensheimer Strasse 48, 66386 Sankt Ingbert, Germany, robert.lemor@ibmt.fhg.de)

Photoacoustics is a hybrid technology based on the photoacoustic effect that detects absorbed photons ultrasonically. Photoacoustic imaging combines ultrasonic resolution with high contrast due to light absorption depending on the physiology of the examined biological tissue. The resolution of conventional systems is not sufficient for in-vitro measurements of small tissue samples or individual cells. In this work, we present a high-resolution photoacoustic microscopy platform based on the SASAM acoustic microscope that allows high resolution imaging on living cells. The system based on an inverted optical microscope consists of a laser source for optical multi wavelength excitation (diode- and solid-state-laser) which emits nanosecond laser pulses with a wavelength in the near infrared spectrum (optical window). It allows the usage of different ultrasound transducers in the frequency range up to $300 \mathrm{MHz}$. In addition to the photoacoustic imaging mode, all common optical modalities are implemented. Pure acoustic imaging mode is used for reference imaging. The developed photoacoustic microscopy platform is a system for high-quality volumetric imaging in different scanning modes. 3D offline reconstruction is possible based on the presented 2D imaging. The system was characterized by in-vitro measurements with a lateral resolution better than $100 \mu \mathrm{m}$ in the confocal mode.

\section{2:40}

3aBBb6. Acoustic signals from gold nanoparticles irradiated with pulsed lasers. Constantin Ungureanu (Faculty of Science and Technology
(TNW), Biophysical Engineering Group (BPE), Twente University, 7500AE Enschede, Netherlands, c.ungureanu@tnw.utwente.nl), Tamara Van Weperen (University Twente, Building Meander, 7500 AE Enschede, Netherlands, t.m.vanweperen@student.utwente.nl), Jeroen Sijl (Physics of Fluids, University of Twente, P.O. Box 217, 7500 AE Enschede, Netherlands, j.sij1@utwente.nl), Raja Rayavarapu (Faculty of Science and Technology (TNW), Biophysical Engineering Group (BPE), Twente University, 7500AE Enschede, Netherlands, R.Rayavarapu@tnw.utwente.nl), Srirang Manohar (University of Twente, Biophysical Engineering Group (BPE), Faculty of Science and Engineering (TNW), P.O. Box 217, 7500 AE Enschede, Netherlands, s.manohar@tnw.utwente.nl), Michel Versluis (Physics of Fluids, University of Twente, P.O. Box 217, 7500 AE Enschede, Netherlands, m.versluis@utwente.nl), Ton G. Van Leeuwen (Univ. of Twente, P.O. Box 217, 7500 AE Enschede, Netherlands, a.g.j.m .vanleeuwen@tnw.utwente.nl)

Photoacoustic imaging is a new imaging technique, which enables imaging of living tissue with high resolution. The technique analyses ultrasound pulses generated when absorbing structures in tissue are irradiated with pulsed light. Due to the phenomenon of plasmon resonance gold nanoparticles possess high optical absorption coefficients which makes them potential contrast agents in photoacoustics. Further, the heat generated around these particles when irradiated with pulsed light can lead to nonlinear effects including bubble formation. The acoustic signals produced by gold nanoparticles in the linear thermoelastic and non-linear regimes are expected to have specific acoustic signatures. In this study, we investigate the acoustic signals generated by two sets of gold spheres having $25 \mathrm{~nm}$ and $60 \mathrm{~nm}$ diameter irradiated by laser pulses with increasing incident fluence rates. We identify and discuss the differences in acoustic signals belonging to the thermoelastic expansion regime and the non-linear regime. Finally, the experimental results are compared with different theoretical models available in literature.

1:00-1:40 Lunch Break 


\section{Invited Papers}

\section{$1: 40$}

3aBBb7. Imaging tissue and wavefront control: Application to acousto-optics and photoacoustics. François Ramaz (Laboratoire Photons et Matière, ESPCI/CNRS, 10 rue Vauquelin, 75231 Paris Cedex 05, France, francois.ramaz@espci.fr), Emmanuel Bossy (Laboratoire Photons et Matière, ESPCI/CNRS, 10 rue Vauquelin, 75231 Paris Cedex 05, France, emmanuel .bossy@espci.fr), Vincent Loriette (Laboratoire Photons et Matière, ESPCI/CNRS, 10 rue Vauquelin, 75231 Paris Cedex05, France, vincent.loriette@espci.fr), Sylvain Gigan (Laboratoire Photons et Matière, ESPCI/CNRS, 10 rue Vauquelin, 75231 Paris Cedex 05, France, sylvain.gigan@espci.fr), Albert-Claude Boccara (Laboratoire Photons et Matière, ESPCI/CNRS, 10 rue Vauquelin, 75231 Paris Cedex 05, France, boccara@optique.espci.fr)

Although optical waves do not exhibit the same ability to be manipulated as e.g. electromagnetic waves up to a few GHz or acoustic waves, for which field emitters-detectors are available, various tools are nevertheless available in the optical domain which open the path to tricks which can be used in the context of sound-light detection. In the purely optical domain, one knows the impact of deformable mirrors led to adaptive optics which is now coupled to OCT for retinal examination. Real time holography leads to complex wavefront synthesis and are useful in acousto-optics (AO) to monitor very complex speckle field distributions. Phase conjugation which is in certain conditions equivalent to time reversal, starts to be used for wavefront retrieval after propagation in turbid media, one can think to use it for AO signal monitoring; moreover matrices of spatial light modulators allow to control light not only spatially but also in the frequency domain and open the field to new astonishing experiments. We will discuss a few applications and perspectives offered by these new devices to the field of biomedical imaging.

\section{2:00}

3aBBb8. Optoacoustics for biomedical sensing, monitoring, and imaging. Rinat O. Esenaliev (University of Texas Medical Branch, 301 University Blvd., Galveston, TX 77555-0456, USA, riesenal@utmb.edu)

Optoacoustics can provide diagnostic information with high optical contrast and ultrasound resolution. It is based on detection of broadband ultrasound induced by short optical pulses and utilizes absorption contrast in tissues. We proposed and developed sensing, monitoring, and imaging optoacoustic systems for in a variety of biomedical applications including pulsed laser tissue ablation, accurate measurement of tissue optical properties, tumor detection, monitoring of tissue hyperthermia, coagulation, freezing, and important blood parameters such as oxygenation and hemoglobin concentration. Recently, we built a novel, portable, multi-wavelength optoacoustic system which includes optical parametric oscillator operating in the range from 680 to $2400 \mathrm{~nm}$ and sensitive, broadband optoacoustic probes developed in our laboratory. We tested the system performance in animal and clinical studies. We monitored continuously and in real time cerebral blood oxygenation and total hemoglobin concentration. Direct probing of blood vessels with high contrast and resolution allowed for monitoring of these parameters with accuracy approaching that of the standard invasive techniques. Results on other important clinical applications of the optoacoustic technique will be presented as well.

\section{Contributed Papers}

\section{2:20}

3aBBb9. Photoacoustic tomography with a single detector in a reverberant cavity. Ben Cox (University College London, Gower Street, London, WC1E 6BT London, UK, bencox@mpb.ucl.ac.uk), Paul C. Beard (University College London, Gower Street, London, WC1E 6BT London, UK, pbeard@medphys.ucl.ac.uk)

In biomedical photoacoustic tomography (PAT), acoustic pulses, generated by the absorption of pulses of near-infrared light, are recorded on an array of ultrasound detectors, and the measured pressure time series are subsequently transformed into an image of the absorbed optical energy density within the tissue. For high resolution imaging, large-area detector arrays with a high density of sensitive, small elements are required. Such arrays can be expensive, so reverberant-field PAT is proposed as a means of obtaining PAT images using smaller arrays or even a single detector. By recording the reflections from a reverberant cavity surrounding the detector(s), in addition to the primary acoustic waves, sufficient information can be captured to allow a PAT image to be reconstructed, without the requirement for a large-area array. Image reconstruction, time reversal, non-uniqueness, and related ideas in quantum chaos will be discussed.

\section{2:40}

3aBBb10. Detection of elastic and optical contrasts in tissue mimicking phantoms based on the interaction of coherent laser light and transient displacements caused by the acoustic radiation force. Khalid Daoudi (Laboratoire Photons et Matière, ESPCI/CNRS, 10 rue Vauquelin, 75231 Paris Cedex 05, France, khalid.daoudi@espci.fr), Emmanuel Bossy (Laboratoire Photons et Matière, ESPCI/CNRS, 10 rue Vauquelin, 75231 Paris Cedex 05, France, emmanuel.bossy@espci.fr), Mickael Tanter (Laboratoire Ondes et Acoustique, ESPCI, Université Paris 7, CNRS, 10 rue Vauquelin, 75005 Paris, France, michael.tanter@espci
.fr),Mathias Fink(Laboratoire Ondes et Acoustique, ESPCI, Université Paris 7, CNRS, 10 rue Vauquelin, 75005 Paris, France, mathias.fink@espci .fr), Albert-Claude Boccara (Laboratoire Photons et Matière, ESPCI/CNRS, 10 rue Vauquelin, 75231 Paris Cedex 05, France, boccara@optique .espci.fr)

We recently demonstrated experimentally the possibility to optically detect transient perturbations generated by the acoustic radiation force, applied several centimetres deep in a tissue-mimicking phantom [Bossy et al., APL 90(17), 2007]. We proposed a detection scheme based on the transient decorrelation (millisecond time scale) of optical speckle patterns formed by coherent laser light that propagates through the phantom during the application of the radiation force. We demonstrated that this approach allows to detect optical contrasts embedded several centimetres deep in the phantom, based on the time-evolution of the correlation coefficient during the transient motion of the medium. In the present work, we demonstrate that this approach also allows the detection of shear mechanical contrast. Moreover, the shape of the observed decorrelation curve provides a way to discriminate between an optical contrast and a shear mechanical contrast. We are now currently investigating the feasibility of detecting with spatial resolution the propagation of a shear wave, based on its effect on localized acousto-optic interaction.

\section{3:00}

3aBBb11. Hybrid photoacoustic - ultrasound transmission parameter imaging in a miniature photoacoustic imager. Srirang Manohar (University of Twente, Biophysical Engineering Group (BPE), Faculty of Science and Engineering (TNW), P.O. Box 217, 7500 AE Enschede, Netherlands, s.manohar@tnw.utwente.nl), Rene 
Willemink(University of Twente, Signals and Systems Group (SaS), Faculty of Electrical Engineering, Mathematics and Computer Sciences (EWI), P.O. Box 217, 7500AE Enschede, Netherlands, G.H.Willemink@utwente .nl), Johan C. Van Hespen (University of Twente, Biophysical Engineering Group (BPE), Faculty of Science and Engineering (TNW), P.O. Box 217, 7500 AE Enschede, Netherlands, J.C.G.vanhespen@utwente.nl), Ton G. Van Leeuwen (Univ. of Twente, P.O. Box 217, 7500 AE Enschede, Netherlands, a.g.j.m.vanleeuwen@tnw.utwente.nl)

Photoacoustic imaging is based on detecting laser pulse induced ultrasound transients from absorbing structures in tissue. The technique combines the advantages of a high optical absorption exhibited by tumors for example with the high resolution possible with ultrasound. A conventional photoacoustic imager operating in a CT geometry comprises a light source, an ultrasound detector array and a coupling medium usually water between the subject under investigation and detector. The imaging protocol usually consists of obtaining projections at angles around the subject. Recently we reported a method of measuring the local speed-of-sound variations by the addition of an absorber to the conventional imager, arranged between the light source and subject. This generates an ultrasound signal which interacts with the subject which can then be detected at the far-end by the detector. This allows the measurement of the integrated speed-of-sound (SOS) at projections around the subject leading to the formation of SOS images. In this article we discuss further analysis of the ultrasound obtained at projections to obtain images of the acoustic attenuation as well. In addition conventional photoacoustic images of the subject are also formed. The principle is demonstrated on well-characterized phantoms and we discuss the potential applications of this technique.

WEDNESDAY MORNING, 2 JULY 2008

ROOM 353, 8:00 TO 10:00 A.M.

\title{
Session 3aEAa
}

\section{Engineering Acoustics and Signal Processing in Acoustics: Transducers and Signal Processing for the Oil and Gas Industry I}

\author{
Fernando Garcìa-Osuna, Cochair \\ Schlumberger, Sugar Land, TX, USA \\ Benoit Froelich, Cochair \\ Etudes et Productions Schlumberger, 1, rue Becquerel, BP 202, Clamart, 92142, France
}

\section{Contributed Paper}

\section{8:00}

3aEAa1. Sonic gas analyzer for hydrogen and methane. Steven Garrett (Penn State, Applied Research Laboratory, PO Box 30, State College, PA 16804, USA, sxg185@psu.edu)

In the late-1800s, Germans used a "methane whistle", known as a Schlagwetterpfeife, to determine the presence of hydrogen or methane in the air that was circulated in mines. This paper describes a modern version of that concept that should be suitable for safety-related applications in mines, sewers, garages that shelter hydrogen or methane-powered vehicles, etc. A compact, self-resonant standing wave tube, $2 \mathrm{~cm}$ in diameter and $3 \mathrm{~cm}$ long, is capped at both ends by two small electrodynamic speakers. The cylindri- cal copper resonator has two slits at the mid-plane to allow a $25 \mathrm{~mm}$ fan to continuously sample the air which is brought to the resonator's temperature after being drawn along the outside of the resonator body. Variation in the resonator's temperature is compensated electronically by an integratedcircuit temperature sensor that produces one microampere per Kelvin. The sensor can measure the concentration of hydrogen to an accuracy of better than $\pm 30 \mathrm{ppm}$ or methane to $\pm 0.02 \%$ in air using phase-locked loop frequency tracking electronics and a voltage-controlled oscillator. Concentration data will be presented for several different gas mixtures and will be compared to concentrations determined by other techniques (e.g., thermal conductivity gauges).

\section{Invited Papers}

3aEAa2. Cylindrical ultrasonic array for borehole applications. Kenneth Liang (Schlumberger-Doll Research, One Hampshire Street, Cambridge, MA 01775, USA, kliang@boston.oilfield.slb.com), Gérard Fleury (Imasonic, 15 rue Alain Savary, 25000 Besançon, France, Gerard.Fleury@imasonic.com), Benoit Froelich (Etudes et Productions Schlumberger, 1, rue Becquerel, BP 202, 92142 Clamart, France, bfroelich@ @ clamart.oilfield.slb.com), Jean-Luc Guey (Imasonic, 15 rue Alain Savary, 25000 Besançon, France, jean-luc.guey@imasonic.com), Pascal Schoeb (Statice Etudes \& Developpement, 9, rue Thomas Edison, 25000 Besançon, France, p.schoeb@statice.com)

A cylindrical ultrasonic array has been developed for operating environments that can reach extremes of $175 \mathrm{C}$ and 20,000 psi. The array is a key component of the PharUSIT* (Phased Array Ultrasonic Transducer for Inspection of Tubing), a research demonstrator developed for borehole applications. The full array consists of 800 elements (10 rings of 80 elements each) and can provide a whole range of beam-forming versatilities and capabilities in 3-D, such as variable focusing, beam steering, electronic scanning, etc, all accomplished without mechanical movements. Special piezo-composites have been developed for the transduction layer, and new polymeric composites have been formulated for the backing material. The center frequency was chosen to be about $500 \mathrm{kHz}$ to accommodate attenuation of the propagation media. A novel technique utilizing custom flexible circuit provides electrical connections between the array and the front-end electronics. Special fabrication processes have been developed to construct the array in a cylindrical geometry. A customized testing protocol has been implemented to demonstrate the survivability of the array technology and to evaluate 
the performance characteristics of individual elements under high-temperature/high-pressure conditions. The unique experimental setup will be presented. Data from electroacoustic measurements such as electrical impedance, bandwidth, sensitivity, angular directivity, and inter-element cross-talks will be shown. *EC GROW project PharUSIT (GIRD-CT-2002-000689)

\section{8:40}

3aEAa3. Acoustic modeling for designing sonic logging tools. Toshihiro Kinoshita (Schlumberger K.K., 2-2-1 Fuchinobe, 2290006 Sagamihara, Japan, tkinoshi@fuchinobe.oilfield.slb.com), Hiroshi Hori (Schlumberger K.K., 2-2-1 Fuchinobe, 229-0006 Sagamihara, Japan, hori@fuchinobe.oilfield.slb.com)

Formation acoustic properties such as velocity and attenuation provide useful information regarding rock type and formation fluid. Sonic logging is one of the principal measurements to evaluate the presence of hydrocarbons in the well and to enable an efficient and safe production of oil. Unfortunately the sonic measurement is affected by the tool itself. Suppressing or attenuating the tool wave, which propagates along the logging tool and is received by the sensors, is directly related to the accuracy of the answer products. In order to improve the measurement quality and to increase the efficiency of logging operations, the structure of sonic logging tools must be well understood. A sonic tool with known effects is decidedly more complex and acoustic modeling incorporating the tool structure details is becoming essential to better sonic tool design. The methodology of numerical modeling used to predict the response of a sonic tool is to be presented.

\section{9:00}

3aEAa4. Extracting dispersion curves of acoustic data with continuous wavelet transform. Henri-Pierre Valero (Schlumberger Doll Research, 1 Hampshire Street - MD- B 213, Cambridge, MA 02139, USA, hvalero@boston.oilfield.slb.com), Sandip Bose (Schlumberger Doll Research, 1 Hampshire Street - MD- B 213, Cambridge, MA 02139, USA, bose1@ boston.oilfield.slb.com), Suchin Aeron (Boston University College of Engineering, One Sherborn Street, Boston, MA 02215, USA, shuchin@bu.edu)

Group and phase velocity (i.e. dispersion) of acoustic data carry important information about the acoustic state of the rock and are important tools for interpretation and for quantitative inversion. In this paper we propose an approach to automatically extract the velocity dispersion (group and phase) of acoustic waves received by an array of receivers without the use of any physical model, user inputs or supervision. The first step consists in applying the continuous wavelet transform to the waveforms recorded by each receiver. Then, the peak on the modulus map is used to determine the time locus of the energy of each mode as a function of frequency. At each frequency, a method of data association is used to find the corresponding loci for all receivers. A straight line is fit to these loci and its slope is used as an estimate for the group velocity at that frequency. Moreover the rate of change of the phase of the complex wavelet coefficients at the locations corresponding to this best-fit straight line can be used to compute an estimate of the phase slowness at the same frequency. Repeating this for all frequencies and all separated modes of interest yields the desired dispersion curves for these modes.

\section{Contributed Paper}

\section{9:20}

3aEAa5. Small scale adaptation of the seismic full waveform inversion method - Application to civil engineering applications. Francois Bretaudeau (LCPC, Centre de Nantes BP4129, 44341 Bouguenais, France, francois.bretaudeau@lcpc.fr), Donatienne Leparoux (LCPC, Centre de Nantes BP4129, 44341 Bouguenais, France, donatienne.leparoux @1cpc.fr), Odile Abraham (LCPC, Centre de Nantes BP4129, 44341 Bouguenais, France, odile.abraham@1cpc.fr)

Full Waveform Inversion (FWI) is a very general multi-parameters quantitative imaging method originally developed to obtain high resolution images of velocities and attenuation in the natural underground media. FWI promises interesting performances for civil engineering applications like cavity detection. Performances of the FWI method in seismic exploration are difficult to quantify because in real experimentations, the properties of the media are not known. Furthermore, characteristics of the source and coupling of receivers are not controlled. In order to quantify the performances of FWI and its adaptability to near subsurface applications, small scale physicals models are realized and a non-contact laser ultrasonic laboratory is dedicated to simulate seismic reflection measurement configurations at ultrasonic scale. Seismograms well reproduce real scale data in terms of waveforms and repartition of energy. A FWI algorithm developed by Geosciences Azur and applied in geological investigation is used. The direct part of the algorithm uses a frequency domain finite difference modelisation. The inverted model is obtained by using a Gauss-Newton method of optimization. Results of inversion of multi-receivers and multi-sources data obtained from simple physical models are presented.

\section{Invited Paper}

\section{9:40}

3aEAa6. Simulating Sonic Scanner responses in an interactive Web-based High Performance Computing environment. Valery Polyakov (Schlumberger Doll Research, 1 Hampshire Street - MD- B 213, Cambridge, MA 02139, USA, valp@computer .org), Henri-Pierre Valero (Schlumberger Doll Research, 1 Hampshire Street - MD- B 213, Cambridge, MA 02139, USA, hvalero@boston.oilfield.slb.com), Dzevat Omeragic (Schlumberger Doll Research, 1 Hampshire Street - MD- B 213, Cambridge, MA 02139, USA, omeragic@boston.oilfield.slb.com), Raymond L. Kocian (Schlumberger Doll Research, 1 Hampshire Street - MD- B 213, Cambridge, MA 02139, USA, rkocian@boston.oilfield.slb.com), Tarek M. Habashy (Schlumberger Doll Research, 1 Hampshire Street - MD- B 213, Cambridge, MA 02139, USA, thabashy@boston.oilfield.slb.com), Jahir Pabon (Schlumberger Doll Research, 1 Hampshire Street - MD- B 213, Cambridge, MA 02139, USA, jahir@boston.oilfield.slb.com)

Sonic Scanner logging tool collects a wealth of data about the geological formation. The drawback of this is that we are now discovering new features on the acoustic logs that have never been observed before; only rigorous modeling can help properly interpret the data. Invariably, it is difficult to learn quickly how to run a modeling code, set the parameters properly, and be able to detect possible errors in the input. In addition, complex modeling requires high power computing resources, which are not always easily accessible the user. To address these problems we developed a multi-tier Web-based log modeling environment where the Sonic Scanner simulator is 
easily accessible from the common Web browser. The user builds the model in an intuitive AJAX-like interface and submits the simulation to a remote High Performance Cluster. The computed waveforms are played back in the browser using Scalable Vector Graphics in a variety of customizable displays. The Web application is easily available to any user with an Internet access. In addition, a programmatically accessible Web service is available to application developers who desire to build their own interpretation applications using the Sonic Scanner simulator engine.

WEDNESDAY MORNING, 2 JULY 2008

ROOM 353, 10:20 A.M. TO 3:40 P.M.

\title{
Session 3aEAb
}

\section{Engineering Acoustics: Ultrasonic Acoustic MEMS I}

\author{
F. Levent Degertekin, Cochair \\ Georgia Institute of Technology, G. W. Woodruff School of Mechanical Engineering, 801 Ferst Dr. NW, Atlanta, GA \\ 30332-0405, USA \\ Dominique Certon, Cochair \\ Lab. LUSSI Inserm U930 CNRS FRE 2448 Univ. François Rabelais, 10, Boulevard Tonnellé, Tours, 37032, France
}

\section{Contributed Paper}

\begin{abstract}
10:20
3aEAb1. Micro-viscometer using acoustic wave absorption in micro-chamber and channel. Sungjoon Choi (Postech, San 31, HyojaDong, Namgu, 790-784 Pohang, Republic of Korea, csjangel@postech .ac.kr), Wonkyu Moon (Postech, San 31, Hyoja-Dong, Namgu, 790-784 Pohang, Republic of Korea, wkmoon@postech.ac.kr), Geunbae Lim (Postech, San 31, Hyoja-Dong, Namgu, 790-784 Pohang, Republic of Korea, limmems@postech.ac.kr)

A micro-viscometer is designed for real-time measurement on the viscosity change of biomaterial including DNA or RNA, etc. It is composed of two chambers and several micro-channels connecting between them. Each chamber has a PZT unimorph membrane for generating and sensing the
\end{abstract}

sound waves. Namely, one is regarded as an actuator, the other as a sensor. Micro-channels, which have the large attenuation of acoustic waves caused by wall effect, are applied to the micro-viscometer for increasing viscosity effect. When the actuator is driven at a resonance frequency under the condition of continuous flowing of liquid through an inlet/outlet hole, the sensor can detect the amplitude differences caused by viscosity change. Therefore, it is possible to measure the viscosity change in real time by detecting the signal change of sensor. The micro-viscometer can be also applied to the integration kit such as LOC (Lab-On a Chip) to detect whether DNA is amplified by micro-PCR (Polymerase Chain Reaction) and it will give a first opportunity for experimentally evaluating the viscosity effect on the propagation of acoustic waves in micro-channel. [Research supported by MRCnd of DAPA]

\section{Invited Paper}

\section{0:40}

3aEAb2. Use of rapid protyping techniques in transducer manufacture. David Hutchins (University of Warwick, School of Engineering, CV4 7AL Coventry, UK, D.A.Hutchins@warwick.ac.uk), Duncan R. Billson (University of Warwick, School of Engineering, CV4 7AL Coventry, UK, drb@eng.warwick.ac.uk), Robert J. Bradley (University of Warwick, School of Engineering, CV4 7AL Coventry, UK, R.J.Bradley@warwick.ac.uk), Kwok S. Ho (University of Warwick, School of Engineering, CV4 7AL Coventry, UK, K.S.Ho@warwick.ac.uk)

This paper will describe the use of a rapid protyping technique, known as micro-sterolithography, for the manufacture of ultrasonic transducers. There are two main types to be described. The first is a MEMS-type capacitive transducer, this being an example of a capacitive micromachined ultrasonic transducer (CMUT). This can be made with all the main structural elements fabricated from polymers, so that no silicon processing is required. Experiments have investigated the properties of these devices, operating in air, water, and in contact with a metal surface. The technique has also been used to make electromagnetic acoustic transducers (EMATs), by forming the main structure from polymers and including a metal coil. These are interesting developments, which may have application to the fabrication of many types of acoustic MEMS devices. 


\section{Contributed Papers}

\section{1:00}

3aEAb3. Design, fabrication and characterisation of piezoelectric micromachined ultrasonic transducers. Anne-Christine HladkyHennion (IEMN, UMR CNRS 8520, avenue Poincaré, BP 60069, 59652 Villeneuve d'Ascq, France, Anne-Christine.Hladky@isen.fr), Didace Ekeom (Microsonics, 39 rue des Granges Galand, 37550 Saint Avertin, France, didace.ekeom@microsonics.fr), Fabrice Duval (IEMN, UMR CNRS 8520, avenue Poincaré, BP 60069, 59652 Villeneuve d'Ascq, France, fa_duval@yahoo.fr), Eric Cattan (IEMN, UMR CNRS 8520, avenue Poincaré, BP 60069, 59652 Villeneuve d'Ascq, France, Eric.Cattan@univvalenciennes.fr)

Micro-machined ultrasonic transducers (MUT) are investigated for phased arrays in high frequency acoustic imaging to overcome resolution and frequency limits of bulk PZT transducers currently used. The advantage of structures on silicon substrate is that transducers could be integrated with the electronics of the system. From a design point of view, finite element (FEM) codes are commonly used to model MUT, but to take into account the fluid surrounding the device radiation, scattering and the inter-mutual acoustic influence of the MUT arrays cells - the boundary element method (BEM) coupled with the finite element method is advantageously used. This paper describes the design, the fabrication and the characterisation of bending mode circular piezoelectric membranes, with operating frequency around $10 \mathrm{MHz}$. The devices are based on a PZT (lead zirconate titanate) film $1 \mu \mathrm{m}$ thick supported by a membrane of polysilicon, $2 \mu \mathrm{m}$ thick and $\mathrm{SiO} 2,500 \mathrm{~nm}$ thick. The PZT thin film was deposited following a sol-gel route. Fully-supported membranes as well as suspended cells are considered with a view to increase the resonant frequency and decrease the cross coupling between cells.

\section{1:20}

3aEAb4. Capacitive micromachined ultrasonic transducers for generation of highly directional sound with a parametric array. Ira $\mathrm{O}$. Wygant (E. L. Ginzton Laboratory, Stanford University, Stanford, CA
94305, USA, iwygant@stanford.edu),Mario Kupnik(E. L. Ginzton Laboratory, Stanford University, Stanford, CA 94305, USA, kupnik@stanford .edu), Jeffry C. Windsor (Applied Research Laboratories, The University of Texas, P.O. Box 8029, Austin, TX 78713-8029, USA, jwindsor@arlut.utexas.edu), Wayne M. Wright (Applied Research Laboratories, The University of Texas, P.O. Box 8029, Austin, TX 78713-8029, USA, wwright@arlut.utexas.edu), Mark S. Wochner (Applied Research Laboratories, The University of Texas, P.O. Box 8029, Austin, TX 787138029, USA, mwochner@mail.utexas.edu), Goksen G. Yaralioglu (E. L. Ginzton Laboratory, Stanford University, Stanford, CA 94305, USA, goksenin@gmail.com), Mark F. Hamilton (Applied Research Laboratories, The University of Texas, P.O. Box 8029, Austin, TX 78713-8029, USA, hamilton@mail.utexas.edu), Butrus (pierre) T. Khuri-Yakub (E. L. Ginzton Laboratory, Stanford University, Stanford, CA 94305, USA, pierreky @ stanford.edu)

Capacitive micromachined ultrasonic transducers (CMUTs) with vacuum-sealed cavities have been fabricated and used to project directional sound using parametric arrays. The wafer bonding approach used to fabricate the CMUTs provides good control over device dimensions, a singlecrystal silicon membrane that has predictable mechanical properties, and the capability to fabricate CMUTs with large-diameter membranes and deep cavities. The fabricated CMUTs are about $8 \mathrm{~cm}$ in diameter and comprise 284 circular membranes, each $4 \mathrm{~mm}$ in diameter. Testing of CMUTs with $40-\mu \mathrm{m}$ and $60-\mu \mathrm{m}$-thick membranes shows they have center frequencies of $46 \mathrm{kHz}$ and $55 \mathrm{kHz}$ and $3-\mathrm{dB}$ bandwidths of $1.9 \mathrm{kHz}$ and $5.3 \mathrm{kHz}$, respectively. With application of DC bias voltages of $380 \mathrm{~V}$ and $350 \mathrm{~V}$ and an AC excitation of $200 \mathrm{~V}$ peak-to-peak, the devices generate effective source levels of $139 \mathrm{~dB}$ and $131 \mathrm{~dB}$ (re $20 \mu \mathrm{Pa}$ ), respectively. We used the CMUT design with a $60-\mu \mathrm{m}$-thick membrane to produce $5 \mathrm{kHz}$ sound at $3 \mathrm{~m}$ with a 6-dB beamwidth of $8.7 \mathrm{deg}$ and a sound pressure level of $58 \mathrm{~dB}$. The results demonstrate that large-area CMUTs, which produce high pressure ultrasound, can be fabricated for transmitting directional sound with parametric arrays. [Work supported by DARPA.]

\section{Invited Papers}

\section{1:40}

3aEAb5. A finite element tool for the analysis and the design of capacitive micromachined ultrasonic transducer (cMUT) arrays for medical imaging. Alessandro Caronti (Dept. of Electronic Engineering, University Roma Tre, via della Vasca Navale, 84, 00146 Roma, Italy, caronti@uniroma3.it), Giosue’ Caliano (Dept. of Electronic Engineering, University Roma Tre, via della Vasca Navale, 84, 00146 Roma, Italy, giosue.caliano@ uniroma3.it), Philipp Gatta (Dept. of Electronic Engineering, University Roma Tre, via della Vasca Navale, 84, 00146 Roma, Italy, gatta@uniroma3.it), Cristina Longo (Dept. of Electronic Engineering, University Roma Tre, via della Vasca Navale, 84, 00146 Roma, Italy, clongo@uniroma3.it), Alessandro Savoia (Dept. of Electronic Engineering, University Roma Tre, via della Vasca Navale, 84, 00146 Roma, Italy, savoia@uniroma3.it), Massimo Pappalardo (Dept. of Electronic Engineering, University Roma Tre, via della Vasca Navale, 84, 00146 Roma, Italy, pappalar@uniroma3.it)

The fabrication technology of capacitive micromachined ultrasonic transducers (cMUTs) is now mature enough to exploit the potential of this new generation of electro-acoustic transducers in the field of diagnostic medical imaging and non-destructive evaluation. Converting a demonstrator into a commercial product often requires many design and process refinements, and sometimes significant modifications to the fabrication process. Because each design-fabrication-and-test cycle is time-consuming and costly, the development of a modeling tool for the computer simulation of cMUTs is an essential aid to reduce the time to market and costs. Much effort has been put by researchers to develop more and more accurate and smart simulation tools, that advanced from the standard equivalent circuit modeling with lumped parameters to more powerful techniques based on the finite element method (FEM). This paper aims to give an overview of the most ordinary modeling approaches used for the simulation of cMUTs. It is shown that the pulse-echo operation of cMUT arrays for medical imaging applications can be analysed by a set of FEM models developed using a commercial software. The important aspects of the cMUT operation, including electro-mechanical-acoustical coupling and nonlinear effects in transmission, are taken into account. 
3aEAb6. Wafer bonded, local oxidation defined, capacitor micromachined ultrasonic transducers for medical applications. Butrus (pierre) T. Khuri-Yakub (E. L. Ginzton Laboratory, Stanford University, Stanford, CA 94305, USA, pierreky@ stanford.edu) , Kwan Kyu Park (E. L. Ginzton Laboratory, Stanford University, Stanford, CA 94305, USA, kwankyup@stanford.edu), Hyunjoo Jenny Lee (E. L. Ginzton Laboratory, Stanford University, Stanford, CA 94305, USA, hyunjoo@ stanford.edu), Mario Kupnik (E. L. Ginzton Laboratory, Stanford University, Stanford, CA 94305, USA, kupnik@ stanford.edu), Omer Oralkan (E. L. Ginzton Laboratory, Stanford University, Stanford, CA 94305, USA, ooralkan@stanford.edu)

We present a technology for making capacitive micromachined ultrasonic transducers (CMUTs) that is based on direct wafer bonding of silicon on insulator wafer (SOI), and where the vertical dimensions and geometry of the CMUT are defined by local oxidation (LOCOS). The advantages of this approach for making CMUTs is the excellent control, no only over the membrane properties, but also over the shape and dimensions of the cavity. We demonstrated the ability to make devices with cavities in the 10s of nanometer range which enables devices up to a frequency of $100 \mathrm{MHz}$. The devices exhibit reduced charging where we measure a voltage shift of 0.03 $\mathrm{V}$ for devices cycled in and out of collapse at $50 \mathrm{~V}$. The devices also have reduced parasitic capacitance as thicker oxides are grown in the regions of the SOI bond to the membrane. These are typically the regions that contribute most to the parasitic capacitance. Finally, because of the reduced charging, it is possible to operate these devices in and out of collapse which results in higher output pressure than devices operating in conventional mode. Hence, these CMUTs are much more predictable and reproducible in their operation and performance.

\section{Contributed Paper}

\section{2:20}

3aEAb7. Acoustic microelectromechanical viscometer. Arthur Ballato (US Army Communications-Electronics R\&D Center, AMSRDCER-CS, Fort Monmouth, NJ 07703-5201, USA, Arthur.Ballato@us.army .mil)

Current dynamic techniques for measuring fluid shear viscosities using quartz, or other piezoelectrics, rely on the resonator surface being exposed to a measurand bath whose extent greatly exceeds the penetration depth of the evanescent shear mode excited by the active element. This configuration allows the effect of the loading parameters to be expressed concisely. Perturbation of the electrical equivalent circuit parameters of the resonator by the fluid loading permits calculation of the mass density - shear viscosity product. In this paper, we explore the interesting, albeit more complicated situation where the separation between the resonator and a confining wall is less than the penetration depth of the fluid occupying the intervening region. It turns out that the resonator perturbation in this case is a sensitive function of the separation. This important fact permits extreme miniaturization, since for gases between $200 \mathrm{~K}$ and $400 \mathrm{~K}$, pressures between 0.01 to $100 \mathrm{~atm}$, and frequencies between $10 \mathrm{MHz}$ and $1 \mathrm{GHz}$, the penetration depth varies from micrometers to nanometers. Variations in the spacing is effected by using a second, nonresonant piezoelectric as the wall. Micro-electro-mechanics (MEMS) versions of viscometers and associated types of fluid sensors are thereby enabled.

\section{2:40-2:00 Lunch Break}

\section{Contributed Papers}

\section{2:00}

3aEAb8. Thin Film Piezoelectric MEMs Devices. Mareike Dr. Klee (Philips Research Laboratories, High Tech Campus 4, 5656 AA Eindhoven, Netherlands, mareike.klee@philips.com), Henk Boots (Philips Research Laboratories, High Tech Campus 4, 5656 AA Eindhoven, Netherlands, henk.boots@philips.com), Biju Kumar (Philips Research Laboratories, High Tech Campus 4, 5656 AA Eindhoven, Netherlands, biju.kumar @ philips.com), Wilco Keur (Philips Research Laboratories, High Tech Campus 4, 5656 AA Eindhoven, Netherlands, wilco.keur@philips .com), Marco De Wild (Philips Research Laboratories, High Tech Campus 4, 5656 AA Eindhoven, Netherlands, marco.de.wild@philips.com), Peter Dirksen (Philips Research Laboratories, High Tech Campus 4, 5656 AA Eindhoven, Netherlands, peter.dirksen@philips.com), Klaus Reimann (NXP Research, HighTech Campus 4, 5656 AA Eindhoven, Netherlands, klaus.reimann@nxp.com), Olaf Wunnicke (NXP Research, HighTech Campus 4, 5656 AA Eindhoven, Netherlands, olaf.wunnicke@nxp .com), Christel Renders (NXP Research, HighTech Campus 4, 5656 AA Eindhoven, Netherlands, christel.renders@nxp.com), Harry Van Esch (Philips Research Laboratories, High Tech Campus 4, 5656 AA Eindhoven, Netherlands, harry.van.esch@nxp.com), Chris Van Heesch (Philips Research Laboratories, High Tech Campus 4, 5656 AA Eindhoven, Netherlands, chris.van.Heesch@philips.com), Georg Schmitz (Medical Engineering, University Bochum, 44680 Bochum, Germany, georg.schmitz@rub .de), Martin Mienkina (Medical Engineering, University Bochum, 44680 Bochum, Germany, martin.mienkina@rub.de), Michal Mleczko (Medical Engineering, University Bochum, 44680 Bochum, Germany, michal .mleczko@rub.de)

Thin film piezoelectric devices, processed in Si-related processes, are attractive for ultrasound transducers and piezoelectric switches. Thin film ultrasound transducer enable large bandwidth $(>100 \%)$, high frequency operation. In piezoelectric micromachined ultrasonic transducers (PMUTs) the ultrasonic waves are generated by flexural motion of the membrane, which is coupled to strain in the piezoelectric film. We have investigated the piezoelectric properties of thin films for ultrasound transducers and piezoelectric switches. Thin film piezoelectric ultrasound transducers as well as piezoelectric switches have been designed, processed and characterized. Thin film piezoelectric devices with excellent quality and reliability have been realized.

\section{2:20}

3aEAb9. Increasing power-efficiency of a micro-machined pump transducer for a parametric transmitting array in air. Haksue Lee (Postech, San 31, Hyoja-Dong, Namgu, 790-784 Pohang, Republic of Korea, jolary@postech.ac.kr), Wonkyu Moon (Postech, San 31, HyojaDong, Namgu, 790-784 Pohang, Republic of Korea, wkmoon@postech.ac .kr)

Parametric array applications in air, such as high directional loudspeaker system, have been studied with a large radiator (generally $\mathrm{D} \geqslant 300 \mathrm{~mm}$ ) to generate high intensive sound beams required for nonlinear interactions. However, conventional transducer requires high electrical power because its electro-acoustic efficiency is too low due to an acoustic impedance mismatch. For mobile applications, a high efficient transducer is required. As an alternative pump transducer, a feasibility of a micro-machined ultrasonic transducer is studied. The efficiency can be improved by applying a thin-film membrane, overcoming the impedance mismatch. The efficiency of micro-machined transducer is simulated with a reduced dynamic model and compared with that of a conventional transducer. Two types of unimorph piezoelectric micro-machined ultrasonic transducers are designed and fabricated as small pump transducers for a ranging sensor and a loudspeaker. The 
electro-acoustic efficiency and dynamic characteristics related with primary wave generation are mainly examined in this work. The radiation characteristics, directional beam patterns and axial pressure distributions, of primary and secondary waves are also measured and compared with theoretical results. Throughout this work, feasibility of the proposed pump transducer is shown, but further work is also required for practical applications. [Research supported by MRCnd and ADD-UD070054AD of DAPA]

\section{2:40}

3aEAb10. Ultraviolet detector based on a surface acoustic wave oscillator system with ZnO-nanostructure sensing material. Tsung-Tsong Wu (Institute of Applied Mechanics, National Taiwan University, No. 1, Sec. 4, Roosevelt Road, 106 Taipei, Taiwan, wutt@ndt.iam.ntu.edu.tw), Wei-Shan Wang (Institute of Applied Mechanics, National Taiwan University, No. 1, Sec. 4, Roosevelt Road, 106 Taipei, Taiwan, dainty@ndt.iam.ntu.edu.tw), Tai-Hsu Chou (Institute of Applied Mechanics, National Taiwan University, No. 1, Sec. 4, Roosevelt Road, 106
Taipei, Taiwan, deshi@ndt.iam.ntu.edu.tw),Yung-Yu Chen(Department of Mechanical Engineering, Tatung University, No. 40, Sec. 3, Chungshan N. Rd., 104 Taipei, Taiwan, yychen@ttu.edu.tw)

Recently, ultraviolet (UV) detectors based on surface acoustic wave (SAW) devices utilizing the acoustoelectric effect have been demonstrated. However, most of presented cases were based on semiconductor sensing films. Sensors with nanostructure sensing material not only possess high sensitivity but also accelerate response and recovery time due to their high surface-to-volume ratio and fast charge diffusion rate. In this paper, $\mathrm{ZnO}$ nanostructure and a dual delay line SAW oscillator system are combined to develop a high precision UV detector. The UV detector is made of $\mathrm{ZnO}$ nanorod on a $1280 \mathrm{YX}-\mathrm{LiNbO} 3$ based SAW device. The operating frequency is around $145 \mathrm{MHz}$. The system is illuminated by a UV source consists of a Xe lamp and a monochromator, resulting in frequency shifts. The frequency shifts related to different light wavelengths and power are measured and discussed. Results show the ZnO-nanostructure based SAW oscillator is a promising candidate for a real-time and high precision UV detector.

\section{Invited Paper}

3:00

3aEAb11. Silicon Micromachined Energy Concentrating Acoustic Transducers for Biomedical Applications. Amit Lal (Cornell University, 118 Phillips Hall, School of Electrical and Computer Engineering, Ithaca, NY 14853, USA, lal@ece.cornell.edu), Abhishek Ramkumar (Cornell University, 118 Phillips Hall, School of Electrical and Computer Engineering, Ithaca, NY 14853, USA, ar339@cornell.edu), Muhamet Araz (Cornell University, 118 Phillips Hall, School of Electrical and Computer Engineering, Ithaca, NY 14853, USA, mka22@cornell.edu)

Silicon's high maximum ultrasonic particle velocity, high thermal conductivity, and low internal loss, make it an ideal material for for high-intensity ultrasonic actuators. We have designed and microfabricated silicon ultrasonic horns, which when actuated at their $\lambda / 2$ longitudinal resonance (using ceramic PZT transducers) concentrate ultrasonic energy and magnify displacements. By incorporating microprobes at the high energy sites we have successfully demonstrated reduction of penetration force on biological tissues. We have also reported cardiac action potential measurement from multiple sites simultaneously within the heart wall by incorporating platinum electrodes on the microprobes. We have reported on mechanical and spatial characterization of seminiferous tubules in the testis in order to extract sperm for artificial insemination. We are currently exploring silicon ultrasonic horns in detecting testicular and prostate cancer in the early stages. In addition, silicon ultrasonic horns can be used for low power ultrasonic pumping and also atomization. By using bulk micromachining techniques an enclosed conduit is opened along the horn. Due to the highly amplified acoustic field at the tip, fluid enclosed in the channel can be atomized from the tip. Reversibly when the tip is immersed in fluid, fluid can also be pumped into the channel due to changing boundary conditions.

\section{Contributed Paper}

\section{3:20}

3aEAb12. Reproducibity and life duration assessment of cMUT transducers. Cyril Meynier (Vermon SA, 180 rue Général Renault, 37000 Tours, France, c.meynier@vermon.com), Dominique Certon (Lab. LUSSI Inserm U930 CNRS FRE 2448 Univ. François Rabelais, 10, Boulevard Tonnellé, 37032 Tours, France, dominique.certon@univ-tours.fr), Franck Teston (Lab. LUSSI Inserm U930 CNRS FRE 2448 Univ. François Rabelais, 10, Boulevard Tonnellé, 37032 Tours, France, franck.teston @ univ-tours.fr), Guillaume Ferin (Vermon SA, 180 rue Général Renault, 37000 Tours, France, g.ferin@vermon.com)

Cmut-based transducers are often expected to be superior in terms of reproducibility to piezoelectric transducers due to the use of microelectronic technology instead of more traditional machining techniques. During the conventional (sacrificial-layer based) production process of cmut transduc- ers, various process steps can display inhomogenities and uncertainty in flow rate, temperature profile, stochiometry, or other aspects, resulting in variations in layers thicknesses, gap height, and material properties, each having an impact on transducer performances. In this study, we choose to use impedance measurement technique as evaluation tool for the stability of the properties of cmut transducers. A large number of cMUT transducers with the same design, were characterized, allowing to compare their properties from wafer to wafer, and according to their position of origin in the wafer. The resistance of cmut transducers to temperature cycling was also investigated, using impedance and optical measurements control before and after a 3-days $+60 /-20^{\circ} \mathrm{c}$ test. The reliability was tested under a longduration electroacoustic measurement, where a cMUT transducer was submitted to periodic large amplitude excitation, and its pulse-echo response recorded for several hours. 


\title{
Session 3aMUa
}

\section{Musical Acoustics and Speech Communication: Interdisciplinary Research on the Science of Singing: A Tribute to Johan Sundberg}

\author{
Annabel J. Cohen, Cochair \\ University of Prince Edward Island, 550 University Ave, Charlottetown, PE C1A 4P3, Canada \\ Sten Ternstrom, Cochair \\ KTH, Department of Speech, Music and Hearing, Lindstedtsvägen 24, S-100 44 Stockholm, Sweden
}

\section{Invited Papers}

\author{
8:00
}

3aMUa1. Listener-oriented motor control: Comparing evidence from speech and singing. Björn Lindblom (Stockholm University, Universitetsvägen 10C, SE10691 Stockholm, Sweden, lindblom@ling.su.se)

Singing and speech make different use of the vocal tract but work in similar ways with respect to how sound production is controlled. A principle that they share is: output-oriented control. This mode of organization implies that the task of the motor system is defined in terms of the desired properties of the output sound, rather than in terms of motor processes. This claim is strongly supported by Johan Sundberg's experimental work on singing and by 'compensatory articulation' and 'hyper-speech', phenomena that remind us that, for speech, output-directed control is also readily acknowledged. Our comparative review of singin and speech will be placed in the context of the current debate on the 'invariance' and true nature of phonetic units: 'articulatory gestures' or 'auditory goals'?

$8: 20$

3aMUa2. Methods for singing voice control and synthesis. Xavier Rodet (Ircam, 1, place I. Stravinsky, 75004 Paris, France, Xavier.Rodet@ircam.fr)

There are various methods to synthesize the singing voice. Whatever the method, in order to obtain high quality voice output, it is essential to respect the precise parameter values for the first three to five formants of the vowels. These parameters depend on many factors including the singer, note, vowel, vocal effort, etc. Articulation of the notes is achieved by variations of fundamental frequency, vibrato, vocal effort and eventually formant characteristics of the vowels. Finally, the succession of consonants and vowels also has to be taken into account and implemented. With the expertise of J. Sundberg, a method called Formant Waveform Synthesis and a program for the control of all the above mentioned variations, except consonants, were developed at Ircam in the 1980s. This program permitted high quality singing voice synthesis but without consonants. Another synthesis method is to is to concatenate units of recorded singing voice. This provides good consonant-to-vowel articulations. But it necessitates to transpose and change the duration of these units which often degrades the output quality and precise control over the formant characteristics is rather difficult. These various methods will be discussed and examples given.

\section{8:40}

3aMUa3. A developmental approach to voice science. Stefanie Stadler Elmer (University of Zürich, Dachsweg 8b, CH - 4410 Liestal, Switzerland, stefanie.stadler@access.uzh.ch)

Vocal development had been studied mostly with a focus on speaking, and only rarely, on singing. Traditional theories on singing development are often based on wrong premises, e.g. eurocentrism, and reliable analyses of singing are missing or selective. A new theory - inspired by the principles of Piaget's theory -, and a new methodology - based on acoustic measures - are proposed. The voice starts to organise at birth, and gradually adapts to the cultural surrounding and its conventions concerning language, music, and social rules. Vocal and musical behaviour are highly adaptive and constructive, and concern two symbolic systems: music and language. The child develops the voice by playing and imitating. The development proceeds from sensorimotor activities towards more and more conscious actions and thoughts. In order to study children's singing, computer aided programs were devised to analyse and represent pitch, timing, pitch qualities, and syllables. This method yields complex configurations of these parameters describing children's song singing. Detailed descriptions allow to reconstruct the strategies children apply to invent or learn new songs. The empirical results from children at various ages demonstrate that the focus on the analysis of the organisation of the vocal expression is a promising research strategy. 
3aMUa4. Observing a chameleon: How to bridge a gap between the voice training and its scientific description. Jaan Ross (Koidu 122-61, 10139 Tallinn, Estonia, jaan.ross@ut.ee), Allan Vurma (Estonian Academy of Music and Theatre, Rävala Puiestee 16, 10143 Tallinn, Estonia, vurma@ema.edu.ee)

An overview of the authors' research on perception and production of singing voice as well as on the methods of voice training is presented. In the first study, a correlation was found for a group of voice students between the duration of training and the strength of the singer's formant, while the tone quality estimates were not significantly higher for students with longer practice years. In the second study we investigated possible acoustical correlates of the 'forward'/'backward' placing of a voice. A 'forward' placed voice may have higher F1, F2 and/or the singer's formant frequencies as well as a higher level of the singer's formant. In the third study we investigated the intonation accuracy in a cappella performance. There are considerable inter-individual differences between performers as to their adjustment of pitch level. The stability of intonation varies significantly both within a single rendition of the vocal exercise as well as between its consecutive renditions. There is a positive correlation between the deviation of a melodic interval from its equally tempered standard value and the number of out-of-tune judgments by the listeners. The dispersion of out-of-tune judgments is considerable, which suggests that listeners might have adopted different criteria for intonation accuracy.

\section{9:20}

3aMUa5. Rate of change of Fo in performance singing. Ronald C. Scherer (Bowling Green State University, Department of Physics and Astronomy, Bowling Green, OH 43403, USA, ronalds@bgnet.bgsu.edu), Nandhu Radhakrishnan (University of Missouri, 316 Lewis Hall, Department of Communication Science and Disorders, Columbia, MO 65211, USA, radhakrishnann @health.missouri.edu), Prakash Boominathan (Sri Ramachandra Medical College \& Research Institute (DU), Dept. of Speech Language \& Hearing Sciences, Porur, Chennai, 600116 TamilNadu, India, Praxb77@yahoo.com), Haidee Tan (Bowling Green State University, 200 Health Center, Department of Communication Disorders, Bowling Green, OH 43403, USA, haideetan@ hotmail.com)

Johan Sundberg has had a strong interest in the vocal behavior of singers of different styles and nationalities. This tribute talk emphasizes the voluntary change of rate of fundamental frequency in performance singing of ornaments and vibrato. The "taan" gestures from Northern Indian classical singing, the "pulse patterns" in Carnatic Southern Indian classical singing, and pitch change in classical western coloratura singing constitute the primary corpus for this study of Fo rate change. Rate of Fo change varied from approximately 20 to $120 \mathrm{ST} / \mathrm{s}$ for soprano pitch change, 8 to $20 \mathrm{ST} / \mathrm{s}$ for the Southern Indian pulse patterns, and 7 to $60 \mathrm{ST} / \mathrm{s}$ for the Northern Indian taan gestures. What these rates depend upon and rates from other ornaments will be discussed, as well as the relation to maximum Fo rates in the literature, performance needs, perceptual characteristics, and control.

3aMUa6. Singing out of tune: Disturbances of vocal performance in the general population. Simone Dalla Bella (Dept. of Cognitive Psychology, University of Finance and Management in Warsaw, Pawia Street 55, 01-030 Warsaw, Poland, sdallabella@ vizja .pl)

Acoustical methods can provide a reliable and objective estimate of singing proficiency in the general population, in terms of pitch and temporal accuracy (e.g., Dalla Bella, Giguère, \& Peretz, 2007). The majority, when asked to sing a well-known song at a slow tempo, are as proficient as professional singers. Nonetheless, some nonmusicians exhibit poor singing. This deficit is mostly limited to the pitch domain and sometimes is not accompanied by impaired perception. More recently singing proficiency was examined in nonmusicians with tasks extending beyond singing familiar melodies. Forty participants imitated single pitches, intervals, and short melodies; in addition, participants sung three well-known melodies at a spontaneous tempo and at a fixed slow tempo. Additional tasks (e.g., Montreal Battery of the Evaluation of Amusia) were carried out to assess participants' perceptual abilities. Acoustical analyses of vocal performance revealed that the majority of nonmusicians sung in tune and in time, thus confirming previous findings. Still, various patterns of poor singing emerged from the analyses of pitch and time accuracy (e.g., poor pitch singing with or without perceptual deficits). The relationship between perception and performance mechanisms in vocal production will be discussed.

\section{0:00}

3aMUa7. Hi-Fi voice: observations on the distribution of energy in the singing voice spectrum above 5 kHz. Sten O. Ternström (Kungliga Tekniska Högskolan, Dept. of Speech, Music \& Hearing, Lindstedtsvägen 24, SE-100 44 Stockholm, Sweden, stern@kth.se)

Current audio technology enables the weak spectrum of the voice above 4-5 kHz to be studied reliably. It is known that energy in the $5-20 \mathrm{kHz}$ range can be perceived even when it is $50 \mathrm{~dB}$ or more below the main voice spectrum peak. Also, these upper frequencies are conventionally emphasized in the production of popular vocal music; yet very few studies of the acoustic content of this range have been made. High fidelity recordings were made of singers sustaining vowels at varying levels of vocal effort. A general characterization of the two highest octaves $(5-20 \mathrm{kHz})$ was sought. The prevalence of high-frequency energy, the variation of harmonics to noise over frequency, and the covariation with overall SPL were all highly variable, but several landmark features were identified. In addition to the commonly observed zero at $4-5 \mathrm{kHz}$, zeroes were often seen also around 6 and $12 \mathrm{kHz}$, as were clusters of resonances in the regions 7-10 kHz and 13-16 kHz. Harmonic energy was observed to over $16 \mathrm{kHz}$ in strong female voices. The audibility of these features was assessed by listening tests with selective filtering. A feature-based nomenclature for these uppermost frequency bands is suggested. 
3aMUa8. Advancing Interdisciplinary Research in Singing through a Shared Digital Repository. Annabel J. Cohen (University of Prince Edward Island, 550 University Ave, Charlottetown, PE C1A 4P3, Canada, acohen@upei.ca)

The domain of research in singing encompasses numerous disciplines, countless styles, and many lifespan stages of skill development. A comprehensive understanding of this domain would benefit from a vast digital repository for storing, accessing, and annotating recordings of singing in all its manifestations. A cross-cultural strategy for acquiring and storing the essential audio, audiovisual, and symbolic data parallels the Child Language Data Exchange System (CHILDES) developed and maintained by Brian MacWhinney (http://childes.psy.cmu.edu/) and used by hundreds of psycholinguistics researchers worldwide. A prototype of a digital repository for singing (http://vre.upei.ca/chimes) is under development using infrastructure at UPEI associated with a Canada Foundation for Innovation project on the enhancement of education through media. The database will support and be supported by an international team aiming to Advance Interdisciplinary Research in Singing (AIRS) on the following themes: (1) acquisition of singing (2) comparison of singing and speech acquisition (3) singing pedagogy (4) singing and intercultural understanding (5) singing and intergenerational understanding (6) singing and well-being. The breadth of the work of Johan Sundberg, an honoured member of this team, encompasses these themes and exemplifies the international, interdisciplinary scope of the initiative. (Supported by Canada Foundation for Innovation CFI).

WEDNESDAY MORNING, 2 JULY 2008

AMPHI MAILLOT, 11:00 A.M. TO 12:40 P.M.

\title{
Session 3aMUb
}

\section{Musical Acoustics: Plucked Stringed Instruments I}

\author{
François Gautier, Cochair \\ Laboratoire d'Acoustique de l'Université du Maine, Avenue Olivier Messiaen, Le Mans, 72085, France \\ Chris Waltham, Cochair \\ University of British Columbia, Department of Physics \& Astronomy, Vancouver, BC V6T 1Z1, Canada
}

\section{Contributed Papers}

\section{1:00}

3aMUb1. A vibrating sitar string: Modeling the 3D dynamics of a plucked string impacting a spatial obstacle with friction. Chandrika P. Vyasarayani (University of Waterloo, Department of Systems Design Engineering, Waterloo, ON N2L 3G1, Canada, cpvyasar@engmail.uwaterloo .ca), Stephen Birkett (University of Waterloo, Department of Systems Design Engineering, Waterloo, ON N2L 3G1, Canada, sbirkett@ real .uwaterloo.ca), John McPhee (University of Waterloo, Department of Systems Design Engineering, Waterloo, ON N2L 3G1, Canada, mcphee@real .uwaterloo.ca)

In some musical instruments of Indian origin, such as sitar, veena, or tambura, the string termination is a shallow curved (parabolic) bridge. The string is usually plucked obliquely and interacts with the bridge, thus producing a buzzing or ringing tone which is significantly different from stringed instruments with knife edge supports like guitar. The string is also not restricted to planar motion and friction between bridge and string plays an important role in damping the non-planar motion. A mathematical model is presented for simulating a $3 \mathrm{D}$ vibrating string as it impacts a curved obstacle with friction. The bridge is represented as a foundation model with nonlinear visco-elastic springs; multiple distributed spatial impacts occur between the bridge and string, however the locations of these are not known in advance so must be determined during the simulation. Galerkin approximation is used to reduce the partial differential equations of motion to a set of nonlinear ordinary differential equations. These equations have been solved numerically for different contact and friction conditions. Parametric studies show the effect of bridge geometry, impact damping, and friction on the bidirectional vibration behavior of the string subject to an oblique plucking idealized as an initial condition.

11:20

3aMUb2. Acoustical measurement of Indian musical instruments (vina-s): Towards greater understanding for better conservation. Sandie Le Conte (Musée de la Musique, 221 avenue Jean Jaures, 75019
Paris, France, sleconte@cite-musique.fr),Stéphane Vaiedelich(Musée de la Musique, 221 avenue Jean Jaures, 75019 Paris, France, svaiedelich @ cite-musique.fr), Philippe Bruguiere (Musée de la Musique, 221 avenue Jean Jaures, 75019 Paris, France, pbruguiere@ cite-musique.fr)

Among the Musée de la musique collection of non-Western musical instruments are some rare and delicate ones from India, which have been the subject of acoustical investigation for a greater understanding, and hence a better conservation. In the case of the vina-s, the vibration of the string (exciter) plucked by the musician is transmitted to the resonators made from gourd through a flat bridge thus giving the instrument a remarkably singular timber. Acoustical and vibrational measurements were applied in order to determine the acoustic properties of resonators. These techniques are based on gauging the radiated acoustical field triggered by a light impact or a frequency sweep. This analysis confirms the importance given by instrument makers to the painstaking choice of gourd resonators. Thanks to the estimation of resonant frequency - and from the vibrating length - it is possible to hypothesise on the type of strings used (the material, diameter and strain). In short, these analyses make it possible the diagnosis of the mechanical state of constituent elements. These descriptors could facilitate decisions to optimise the choice of gourds in the production of instruments (sitar, tampura) that have today taken on semi-industrial shades.

\section{1:40}

3aMUb3. The influence of plectrum thickness on the radiated sound of the guitar. Sandra Carral (University of Music and performing Arts, Anton-von-Webern-Platz 1, Gebäudeteil M, 2. Stock, A-1030 Vienna,Austria, carral@mdw.ac.at), Martin Paset (University of Music and performing Arts, Anton-von-Webern-Platz 1, Gebäudeteil M, 2. Stock, A-1030 Vienna, Austria, martin.paset@students.mdw.ac.at)

It is generally thought that the human ear is very sensitive to subtle changes in sounds. In the context of musical instruments, one important aspect to study is how much the physical attributes of any given instrument have to differ so that a human can perceive a difference in the produced 
sound. In the case of the guitar, it is unquestionable that when the string is plucked at a particular position, some of the differences on the produced sound of the instrument are introduced by changes in how the player plucks the string (playing technique). However, given the wide variety of plectrum types, materials and thicknesses, it is hypothesised that the player is not the only parameter that influences the sound, but that the plectrum itself plays a significant role in the sound production. This paper presents a study whereby a guitar is played with three plectra of different thicknesses with an artificial plucking machine. The radiated sound is recorded, and subsequently analysed using the program SNDAN. Physical and psychoacoustical attributes of the sound are calculated from the resulting analysis. A thorough comparison of these results obtained for the three different plectra is presented and discussed.

\section{2:00}

3aMUb4. Acoustics of the French Baroque Lute. Nicholas Makris (Massachusetts Institute of Technology, Room 5-212, 77 Massachusetts Avenue, Cambridge, MA 02139, USA, makris@mit.edu)

Design innovations of the French baroque lute characterize the final plateau in the lutes' millennia long evolution in Europe before its eventual extinction at the end of the baroque period. They came with a dramatic increase in number of strings, a new tuning, the first physical description of string vibration, by Marin Mersenne, and led to the "style brisee" which became ubiquitous in baroque music. Compared to the violin, the lute experienced relatively large variations in design and construction material over its lifetime. The aesthetic effect of these variations on musical acoustics were and are often described in compelling but qualitative terms, even by players such as Aristotle and Constantjin Huygens. Here we will use fundamental theoretical principles to quantitatively analyze the acoustic effects of typical design and construction variations found in historic instruments, ranging from the material and thickness of sound boards, bracing and ribs, to rosette design and stringing. We will show how quantitative analysis can be reconciled with qualitative descriptions to help match lute design with desired sound quality.

\section{2:20}

3aMUb5. Vibrational Characteristics of Harp Soundboards. Chris Waltham (University of British Columbia, Department of Physics \& Astronomy, Vancouver, BC V6T 1Z1, Canada, cew@phas.ubc.ca)

The musical quality of a harp depends on many factors, but key among these is the soundboard. This work sets out to define general vibrational qualities of a good harp soundboard. First, in order to understand the relationship between the vibrational behavior of a bare soundboard and that of the completed instrument, a 36-string harp was built from scratch. Measurements were made at each stage of construction and compared to finiteelement calculations. The results showed how the bare soundboard properties affect those of the finished harp. Second, the soundboards of several harps of different sizes were assessed by measuring the admittances along the string bar. These data, and the views of the musicians who play the harps, showed that the most crucial relationship is that between the modal shapes and frequencies of the soundboard, and the position and pitches of the strings attached to it. This allows a general statement to be made about the vibrational qualities of a good soundboard, and suggests a recipe for improving poor soundboards.

WEDNESDAY MORNING, 2 JULY 2008

ROOM 250A, 8:00 TO 9:40 A.M.

Session 3aNSa

\title{
Noise, Physical Acoustics, and EURONOISE: Aeroacoustics IV
}

\author{
Philip J. Morris, Cochair \\ Penn State University, 233C Hammond Building, University Park, PA 16802, USA \\ Christophe Bailly, Cochair \\ Ecole Centrale de Lyon, 36, avenue Guy de Collongue, LMFA, Ecully, 69134, France
}

\section{Contributed Papers}

\begin{abstract}
8:00
3aNSa1. Finite Volume Solvers and Moving Least Square Approximations for the Linearized Euler Equations on Unstructured Grids. Sofiane Khelladi (Lab. d'Energétique et de Mécanique des Fluides Interne, Arts et Métiers ParisTech, 151 boulevard de l'Hôpital, 75013 Paris, France, sofiane.khelladi@paris.ensam.fr), Xesús Nogueira (Group of Numerical Methods in Engineering, GMNI Dept. of Applied Mathematics, School of Civil Engineering, University of A Coruña, Campus de Elviña, 15071 A Coruña, Spain, xnogueira@udc.es), Farid Bakir (Lab. d'Energétique et de Mécanique des Fluides Interne, Arts et Métiers ParisTech, 151 Boulevard de l'Hôpital, 75013 Paris, France, farid.bakir @ paris.ensam.fr), Luis Cueto-Felgueroso (Aerospace Computational Design Lab., Dept. of Aeronautics and Astronautics, Massachusetts Institute of Technology, 77 Massachusetts Avenue, Cambridge, MA 02139, USA, lcueto@mit.edu), Ignasi Colominas (Group of Numerical Methods in Engineering, GMNI Dept. of Applied Mathematics, School of Civil Engineering, University of A Coruña, Campus de Elviña, 15071 A Coruña, Spain, icolominas@udc.es)
\end{abstract}

This paper presents the application of a high-order finite volume scheme based on the Moving Least Squares approximations (FVMLS) to solve Linearized Euler Equations (EEL) on unstructured grids. The proposed method allows the direct reconstruction of the (convective) fluxes using compact stencils, and without introducing new degrees of freedom, which is a real advantage over the most popular existing high-order methods. This particularity confers to the proposed methodology a significant reduction in workload and memory storage capability. The proposed scheme is compared to a second-order accurate finite volume scheme with centered fluxes around a uniform flow, with absorbing and/or reflecting boundary conditions. Some test cases are used to illustrate the potential of the FVMLS approximations on acoustic wave propagation. The ultimate objective of our research action is to simulate acoustic wave propagation into confined geometries and complex flows provided by subsonic turbomachines.

\section{$8: 20$}

3aNSa2. Weak Compressibility Method for Prediction of Fluid Noise Generation by Cavity Flow at Low Mach Number. Ming Zhu (ESI Group, 99 rue des Solets, BP 80112, 94513 Rungis, France, Ming.Zhu @ esi-group.com), Kjell Herfjord (Statoil Hydro, P.O. Box 7190, 5020 Bergen, Norway,kjher@statoilhydro.com), Mustafa Megahed (ESI Group, Mergenthalerallee 15-21, 65760 Eschborn, Germany, mme@esi-group 
.com),Argiris Kamoulakos(ESI Group, 99 rue des Solets, BP 80112, 94513 Rungis, France, Argiris.Kamoulakos@esi-group.com)

This paper presents the implementation and validation of a numerical simulation method that takes into account the air-borne acoustic wavepropagation within the turbulent flow solution at low Mach number, which is so-called weak compressibility, in order to cope with fully coupled aeroacoustics problems in a nonlinear system. An academic single 3D cavity case is used to validate the method in comparison with the experiment. The simulated results showed excellent agreement with the experimental data in regard to the acoustic pressure and frequency for the dominant peak of the sound pressure level (SPL) spectra over a wide speed range up to 55 meter per second. An industrial case of so-called singer riser in the system of ocean natural gas rig is studied using this method. There is fluid sound generation due to the interaction of the gas flow field with the pipe grooves. This sound causes strong resonance and vibration over the equipments on the gas rig platform therefore forces the gas producer to control the gas production rate. By using numerical simulation, the aero-acoustic behaviour of a gas flowing in a lab-scale corrugated pipe has been captured which provides deep insights of fluid sound generation mechanism of this singer riser. Experimental results are used to validate the numerical models.

\section{8:40}

3aNSa3. Transparent boundary condition for acoustic propagation in lined guide with mean flow. Emmanuel Redon (Université de Bourgogne, LRMA, 9, avenue Alain Savary, 21000 Dijon, France, Emmanuel .Redon@u-bourgogne.fr), Boureima Ouedraogo (Université de Bourgogne, LRMA, 9, avenue Alain Savary, 21000 Dijon, France, Boureima .Ouedraogo@u-bourgogne.fr), Anne-Sophie Bonnet-Ben Dhia (CNRS, ENSTA - 32 Boulevard Victor, 75015 Paris, France, Anne-Sophie.Bonnet -Bendhia@ensta.fr), Jean-Francois Mercier(CNRS, ENSTA - 32 Boulevard Victor, 75015 Paris, France, jmercier@ensta.fr), Colin Chambeyron (CNRS, ENSTA - 32 Boulevard Victor, 75015 Paris, France, colin.chambeyron@ensta.fr)

A finite element analysis of acoustic radiation in an infinite lined guide with mean flow is studied. In order to bound the domain, transparent boundary conditions are introduced by means of a Dirichlet to Neumann (DtN) operator based on a modal decomposition. This decomposition is easy to carry out in a hard-walled guide. With absorbant lining, many difficulties occur even without mean flow. Since the eigenvalue problem is no longer selfadjoint, acoustic modes are not orthogonal with respect to the L2-scalar product. However, an orthogonality relation exists which permits writing the modal decomposition. For a lined guide with uniform mean flow, modes are no longer orthogonal but a new scalar product allows us to define the DtN operator. We consider first the case of an infinite rectangular two- dimensional lined guide with uniform mean flow in order to present the methodology. Then, some extensions will be presented: non-uniform twodimensional geometries by calculating potential mean flow, and cylindrical axisymmetric three-dimensional problems with uniform mean flow.

\section{9:00}

3aNSa4. Sound radiation from annular jet ducts with impedance walls. João M. Oliveira (Instituto Superior Tecnico, Av. Rovisco Pais, 1049-001 Lisboa, Portugal, joliveira@ist.utl.pt)

An analytical model for sound radiation from a semi-infinite annular duct with cylindrical infinite centre section and carrying a jet issuing into an uniform mean flow is presented. The interior of the outer wall of the duct is allowed to have an acoustic impedance. This is an extension to a recent model where only rigid walls were considered. The unstable cylindrical vortex layer attached to the edge of the duct, between the jet and the uniform mean flow, is considered. The geometry considered aims to model a turbofan exhaust, where sound generated at the inlet propagates along the annular duct and refracts through the bypass jet before radiating into the far field. In this paper the effect of the acoustic impedance of the duct outer wall on the radiated acoustic field is studied.

\section{9:20}

3aNSa5. Acoustics of Low Mach Number Nozzles with Area Expansions. Fernando J. Lau (Instituto Superior Tecnico, Av. Rovisco Pais, 1049-001 Lisboa, Portugal, lau@ist.utl.pt), João M. Oliveira (Instituto Superior Tecnico, Av. Rovisco Pais, 1049-001 Lisboa, Portugal, joliveira@ist.utl.pt)

The acoustic wave equation for quasi-one-dimensional propagation is obtained, along a cylindrical duct with a significant area expansion and containing a low Mach number mean flow. The motivation is the study of the reflection in cross-sectional expanding area. The ray approximation, which holds only for wavelengths which are short compared with the length scales of the variation of the cross-section and mean flow velocity, is used as a factor to reduce the wave equation to a Schrodinger form, in a nozzle with a hyperbolic tangent expansion. The exact solutions are obtained, without restriction, as power series solutions around the middle of the duct; since this solution fails to converge at the two ends of the duct it is matched to the other solutions there, by application of the causality principle. In this way it is possible to calculate everywhere the pressure and velocity perturbation profiles, for both the transmitted and reflected waves, as well as the reflection and transmission coefficients. These are plotted as a function of the longitudinal coordinates along the duct for several values of the three dimensionless parameters in the problem, viz. (i) the area expansion ratio, ii) the Mach number of the mean flow at the central section and iii) the wavenumber. 


\title{
Session 3aNSb
}

\section{Noise and EURONOISE: Railway Noise and Vibration III}

\author{
Richard A. Carman, Cochair \\ Wilson, Ihrig \& Associates, 5776 Broadway, Oakland, CA 94618, USA \\ Franck Poisson, Cochair \\ SNCF DIR, 45, rue de Londres, Paris, 75379, France \\ David Thompson, Cochair \\ ISVR, University of Southampton, Highfields, SO17 1BT, UK
}

\section{Contributed Papers}

\section{8:00}

3aNSb1. Noise reduction of freight wagons achieved by LL-blocks implementation: Last investigations and results from IPG and NICOBB projects. Fabien Létourneaux (SNCF - Agence d'Essai Ferroviaire, 21 avenue Salvador Allende, F-94407 Vitry-Sur-Seine, France, fabien .letourneaux@sncf.fr), Jasper Peen (Lloyd's Register Rail Europe B.V., Catherijnesingel 33, PO Box 2016, 3500 GA Utrecht, Netherlands, jasper.peen@1rrail.com), Nicolas Meunier (Deutsche Bahn AG, Messungen Akustik (TZV12.1), Völckerstraße 5, D-80939 München, Germany, Nicolas.Meunier@bahn.de), Erwin H. Jansen (TNO Science and Industry, PO Box 155, 2600 AD Delft, Netherlands, erwin.jansen@tno.nl), Cora Cremezi-Charlet (UIC, 16,rue J. Rey, F-75015 Paris, France, cremezi@uic .asso.fr)

The high noise emission levels of freight wagons during rolling are mainly due to uneven wheel treads produced during the braking sequences when the vehicle is equipped with cast iron blocks. This has lead to the development of two types of composite brake shoes (K- and LL-blocks), which are far less aggressive to the wheel.It has been demonstrated that K-blocks achieve a noise reduction of about $10 \mathrm{~dB}$ but they are mainly dedicated, at term, to the new vehicles as their friction coefficient is very different to the cast iron one. LL-blocks are on the contrary especially designed for retrofit vehicles and do not require major modifications of the braking equipment. But as they were only very recently made available by the manufacturers due to difficulties in their development, noise efficiency of LLblocks products is not well-established. This paper focuses on the noise reduction and noise emission values of freight vehicles equipped with LLblocks. It aims at presenting on-going studies on this topic and at providing reliable and accurate results from different measurement campaigns carried out on a TSI reference track. This work is the result of a collaboration between the UIC project NICOBB and the Dutch Noise Innovation Programme.

\section{8:20}

3aNSb2. Measurements of curve squeal from Oslo's subway. Sigmund Olafsen (Brekke \& Strand akustikk as, Hovfaret 17, N-0275 Oslo, Norway, so@bs-akustikk.no), Tore Killengreen (Brekke \& Strand akustikk as, Hovfaret 17, N-0275 Oslo, Norway, tkn@bs-akustikk.no)

Curve squeal from the subway line at Brattlikollen, Oslo, has led to severe noise complaints through the years. Two important steps have been taken to reduce the problem. An automatic rail lubrication system has been installed on the critical spot, and Oslo's old subway trains (1300 series) are being replaced. One of the new trains (MX) has also been fitted with wheel dampers. Continuous measurements during 16 days and nights with 2238 train passages have been analyzed to verify the effectiveness of these steps to reduce noise. The main results are as follows: 1300 series trains without rail lubrication give severe curve squeal during $18 \%$ of the train passages. 1300 series trains with rail lubrication give severe curve squeal during less than $1 \%$ of the train passages MX trains give substantially less curve squeal than 1300 series trains, with or without lubrication The MX trains with wheel dampers gives no curve squeal, even without lubrication

\section{8:40}

3aNSb3. Reduction of Noise from Composite Steel/Concrete Aerial Structures by Damping Steel Plates. George P. Wilson (Wilson, Ihrig \& Associates, Inc., 5776 Broadway, Oakland, CA 94618, USA, gwilson @ wiai.com), Francis Kirschner (FGK Consulting, Inc., East Hills, Long Island, NY 11576, USA, francis516@aol.com)

Noise radiated from steel girders at bridges and aerial structures has been a long standing problem for steel wheel and rail systems. During the initial design period for the San Francisco Bay Area Rapid Transit System, a full scale model girder and deck was constructed in 1964 and tested with and without constrained layer damping on the steel girder plates. The radiated noise from $20 \mathrm{~Hz}$ to $150 \mathrm{~Hz}$ was reduced by $9 \mathrm{~dB}$ with full plate area damping. A test track installation in 1965 also showed about $9 \mathrm{~dB}$ reduction in wayside noise with the overall result within about $1 \mathrm{~dB}$ of the noise radiated by an all-concrete structure. Because full plate area constrained damping is expensive, a scheme with small area damping pads was developed in the 1970's and applied to composite steel/concrete aerial structures at the Atlanta rail transit system. The result was 9 to $10 \mathrm{~dB}$ reduction of low frequency radiated noise, allowing sound barrier walls at the outer edges of the decks to be fully effective in reducing overall wayside noise levels from trains. Drawings of the aerial structures and graphical results from vibration and noise tests are presented.

\section{9:00}

3aNSb4. Noise reduction applied to an engine cooling system for a diesel railway vehicle. Anders Frid (Bombardier Transportation, Östra Ringvägen 1, SE-72173 Vasteras, Sweden, anders.r.frid@se.transport .bombardier.com), Karl-Richard Fehse (Bombardier Transportation, Am Rathenaupark, 16761 Hennigsdorf, Germany, karl-richard.fehse@de .transport.bombardier.com)

In recent years there there has been growing attention to reduce the noise from rail traffic in urban areas. In or around stations and depots, noise from cooling systems and traction and auxiliary equipment normally dominates. New legislation setting noise limits for stationary and accelerating trains puts further emphasis on increased noise reduction of such sources. An experimental investigation has been carried out in which several design changes on a roof-mounted diesel engine cooler from the AGC (Autorail Grande Capacité) train have been assessed in laboratory and on a train. Even though this train is considered as a state-of-the-art product having very low noise emission, the engine cooler is identified as the dominating noise source when operating at its maximum capacity. The wide range of minor and major modifications tested includes different ways to guide the air flow into the fans, reduced tip clearance, absorption material in various positions 
inside and outside the cooler. Also different fan types have been tried (an axial fan with swept blades and a radial fan). The work has been carried out within the 6th framework EU-project "Silence" (ref TIP4-CT-2005-516288)

\section{9:20}

3aNSb5. Upgraded BB64700 locomotives - Noise Management Plan during the redesign process. Sebastien Dalle (CETIM, 52, av. Felix Louat, 60300 Senlis, France, sebastien.dalle@cetim.fr)

To extend the working life of its BB 64700 locomotives, SNCF decided to upgrade a fleet of 19 tractive units. Priorities for the upgrading work include changing the motor and its cooling system, adding air conditioning to the cab while meeting new environmental obligations and improving cab acoustics. An acoustic model that combines calculations and experimental data is used as a platform to specify maximum allocated acoustic power to each system suppliers. The global approach to sub-structuring and acoustic synthesis is based on decomposition of the product in a main structure and several noise sources, and that their acoustic and vibration energy propagates via different identified transfer paths. The model, which is updated throughout the design process, serves as a decision tool to enable corrective action plans if a clause might be not respected. In the context of this project, measured noise levels on the upgraded locomotive meet SNCF specifications for interior and exterior acoustic according to the defined operating conditions (idling, max load traction).

\section{9:40}

3aNSb6. Noise reduction strategies for a light metro rail traction system. Nicola Rusciano (Dept. of Aerospace Engineering - University of Naples, Via Claudio, 21, 80125 Naples, Italy, nruscian@unina.it), Massimo Viscardi (Dept. of Aerospace Engineering - University of Naples, Via Claudio, 21, 80125 Naples, Italy, massimo.viscardi@unina.it), Stefano Ferraiuolo (Ansaldobreda, Via Argine, 80100 Naples, Italy, ferraiuolo .stefano@ansaldobreda.it)

Inside the paper the vibro-acoustic characterization of a light rail vehicle is presented, with special reference to the traction noise emission identification. As a first step, on board measurements and pass by measurements, were performed in order to separate traction noise from rolling noise; these test revealed the electrical engine, as the most relevant sources at low speed. The electrical engine has then been characterized during specific laboratory test and innovative noise control solution were proposed to be tested. The first one could be a modification of inverter algorithm; this trying to reduce the influence of most relevant acoustic tones. This approach is essentially based on a software modification of the PWM algorithm through which modify the engine's spectral response. The second possible tested approach involved the enclosure of the engine in a sort of semi-active skin panels co-located at short distance from the engine; it has been demonstrated that this approach, by the merging of passive and active peculiarities, potentially lead to a wide frequency range application area. Following laboratory tests, the PWM solution was also decided to be implemented during "on board" test; a wide set of experimental results will be presented and discussed.

\section{0:00}

3aNSb7. Tram noise emission: Spectral analysis of the noise source contributions. Marie-Agnès Pallas (INRETS, 25 av. F. Mitterrand, case 24, 69675 Bron, France, marie-agnes.pallas@inrets.fr), Joel Lelong (INRETS, 25 av. F. Mitterrand, case 24, 69675 Bron, France, lelong@inrets.fr), Roger Chatagnon (INRETS, 25 avenue F. Mitterrand, case 24, 69675 Bron, France, roger.chatagnon@inrets.fr)

In France we can assist to a wide development of tram networks for public transportation in main cities. At the same time arises questioning on the noise emission of trams and possible consequences for resident exposure. A French research project has been conducted for studying noise and vibration emission of trams : this paper concerns the description of the noise emission of passing-by trams. A measurement campaign was achieved on the city of Nantes network, involving two kinds of trams with distinct technologies and equipment, and tested on two types of tracks having different platform surfaces. Acoustic measurement included both a 2D-array for noise source identification and a microphone set for vertical directivity analysis. The dominant noise sources are mainly the bogie areas (powered bogies, unpowered bogies) and an extended noise source along the track and the lower part of the tram, all of them involving rolling noise. This paper focuses on the spectral description of the main sources. A parametric study is presented, pointing out the effect of speed, tram type and track type on the frequency distribution of the emitted noise. 


\title{
Session 3aNSc
}

\section{Noise, Computational Acoustics, and EURONOISE: Source Identification in Radiation and Scattering I}

\author{
J. Stuart Bolton, Cochair \\ Ray W. Herrick Labs., School of Mech. Eng., Purdue University, 140 S. Martin Jischke Drive, West Lafayette, IN 47907-2031, \\ USA \\ Peter Juhl, Cochair \\ Institute of Sensors, Signals and Electrotechnics, University of Southern Denmark, Niels Bohrs Allé 1, Odense S, 5230, \\ Denmark
}

Invited Paper

8:00

\begin{abstract}
3aNSc1. Spherical near field acoustic holography with microphones on a rigid sphere. Finn Jacobsen (Acoustic Technology Department, Technical University of Denmark, Ørsted Plads, B352, DK-2800 Lyngby, Denmark, fja@oersted.dtu.dk), Jørgen Hald (Brüel \& Kjær Sound \& Vibration Measurement A/S, Skodsborgvej 307, DK-2850 Nærum, Denmark, JHALD@bksv.com), Efrén Fernandez (Acoustic Technology Department, Technical University of Denmark, Ørsted Plads, B352, DK-2800 Lyngby, Denmark, efrenfer@hotmail.com), Guillermo Moreno (Acoustic Technology Department, Technical University of Denmark, Ørsted Plads, B352, DK-2800 Lyngby, Denmark, s051216@student.dtu.dk)
\end{abstract}

Spherical near field acoustic holography (SNAH) is a recently developed technique that makes it possible to reconstruct the sound field inside and just outside an acoustically transparent spherical surface on which the sound pressure is measured with an array of microphones with negligible scattering. Because of the versatile geometry of a sphere SNAH is potentially extremely useful for source identification. On the other hand a rigid sphere is somewhat more practical than an open sphere, and it is possible to modify the SNAH theory so that a similar sound field reconstruction can be made with an array of microphones flush-mounted on a rigid sphere. However, this approach is only valid if it can be assumed that the sphere has a negligible influence on the incident sound field, in other words if multiple scattering can be ignored, and this is not necessarily a good assumption when the sphere is close to a radiating surface. This paper describes the modified SNAH theory and examines the matter through simulations and experimentally.

\section{Contributed Paper}

8:20

3aNSc2. Applications of Near-field Acoustic Holography on Small Apertures. Rick Scholte (Eindhoven University of Technology, Dept. of Mechanical Engineering, Dynamics \& Control, P.O. Box 513, 5600MB Eindhoven, Netherlands, r.scholte@tue.nl), Ines Lopez (Eindhoven University of Technology, Dept. of Mechanical Engineering, Dynamics \& Control, P.O. Box 513, 5600MB Eindhoven, Netherlands, i.lopez@tue.nl), N.b. Roozen (Eindhoven University of Technology, Dept. of Mechanical Engineering, Dynamics \& Control, P.O. Box 513, 5600MB Eindhoven, Netherlands, n.b.roozen@tue.nl), Henk Nijmeijer (Eindhoven University of Technology, Dept. of Mechanical Engineering, Dynamics \& Control, P.O. Box 513, 5600MB Eindhoven, Netherlands, h.nijmeijer@tue.nl)

Small, finite measurement apertures are a major impediment for accurate and fast application of near-field acoustic holography (NAH). In theory, FFT-based NAH methods are very powerful and acquire extremely fast in- verse calculations of the sound field. However, in practice the usually small amount of sensors in a measurement array limit the possibilities of classical implementations of NAH, especially near the edges of the array or measurement grid. An alternative method to, for example, spatial windowing, patch $\mathrm{NAH}$, SONAH, etc. is the recently introduced border-padding technique. This method makes it possible to apply FFT-based NAH with a large increase of accuracy compared to the original methods, specifically at the measurement grid edges, while maintaining the high speed of the inverse process PNAH is known for. This work focuses on the application of borderpadding and the practical implementation in a newly developed PNAH system. It illustrates a large variety of extended possibilities for PNAH that border-padding enables. Apart from the earlier introduced standard borderpadding, experimental results are shown for a highly improved borderpadding method. Measurements and calculations on industrial products illustrate the wide applicability of the presented method.

\section{Invited Papers}

3aNSc3. A study on regularization parameter choice in Near-field Acoustical Holography. Jesper Gomes (Brüel \& Kjær Sound and Vibration Measurement A/S, Skodsborgvej 307, DK-2850 Nærum, Denmark, jgomes@bksv.com)

Regularization plays an important role in Near-field Acoustical Holography (NAH), and choosing the right amount of regularization is crucial in order to get a meaningful solution. An automated method such as the L-curve or Generalized Cross-Validation (GCV) is often used in NAH to choose a regularization parameter. These parameter choice methods (PCMs) are attractive, since they require no a priori knowledge about the noise. However, there seems to be no clear understanding of when one PCM is better than the other. This paper presents comparisons of three PCMs: GCV, L-curve and Normalized Cumulative Periodogram (NCP). The latter method is new within NAH and it is based on the Fourier transform of the residual vector. The methods are used in connection with three NAH methods: Statistically Optimized Near-field Acoustical Holography (SONAH), the Inverse Boundary Element Method (IBEM), and the 
Equivalent Source Method (ESM). All combinations of the PCMs and the NAH methods are investigated using simulated measurements with different types of noise added to the input. Finally, the comparisons are carried out for a practical experiment. This aim of this work is to create a better understanding of which mechanisms that affect the performance of the different PCMs.

\section{9:00}

3aNSc4. Regularization method applied to deconvolution problem in Real-Time Acoustic Holography. Sebastien Paillasseur (ENSIM - LAUM, Université du Maine, rue Aristote, 72085 Le Mans, France, sebastien.paillasseur.etu@univ-lemans.fr), Jean-Hugh Thomas (ENSIM - LAUM, Université du Maine, rue Aristote, 72085 Le Mans, France, jean-hugh.thomas@univ-lemans .fr), Jean-Claude Pascal (ENSIM - LAUM, Université du Maine, rue Aristote, 72085 Le Mans, France, Jean-Claude.Pascal@univlemans.fr)

Near-field Acoustic Holography (NAH) [Maynard et Williams, 1985] is a measuring process for locating stationary sound sources from measurements made by an antenna of microphones positioned near the acoustic source plane. In order to characterize nonstationary sources, a new formulation has been introduced [Grulier, 2004] to propagate signals on a forward plane using a convolution product with an impulse response in the time-wavenumber domain. The purpose of this study is to solve the deconvolution problem in order to introduce the Real-Time Acoustic Holography and to test its accuracy. Taking the evanescent waves into account improves the spatial resolution of the solution but makes the deconvolution problem "ill-posed". Thus, the inverse of the impulse response is neither unique nor stable. Then, regularization methods that consist of giving an other constraint to the solution to solve this problem are studied. In particular, the standard Tikhonov regularization is used, which is based on the minimisation of the solution's energy, combined with generalized cross validation to estimate the regularization parameters. This method provides an image representation of the time dependent pressure of the source plane in order to localize and characterize the different sound sources.

\section{9:20}

3aNSc5. A comparison of SONAH and IBEM for near-field acoustic holography. Peter Juhl (Institute of Sensors, Signals and Electrotechnics, University of Southern Denmark, Niels Bohrs Allé 1, 5230 Odense S, Denmark, pmjuhl@ sense.sdu.dk), Jesper Gomes (Brüel \& Kjær Sound and Vibration Measurement A/S, Skodsborgvej 307, DK-2850 Nærum, Denmark, jgomes@bksv.com)

Among the popular techniques for acoustic source identification in complex environments are the Statistically Optimal Near Acoustic Holography (SONAH) and the Inverse Boundary Element Method (IBEM). These two methods are quite different regarding the underlying assumptions and the practical implementations: Whereas SONAH performs the back-propagation of the sound field to a plane surface; the IBEM has no restrictions on the radiating geometry. On the other hand, IBEM requires the generation of a surface mesh and a time consuming solution process. The present paper compares the performance of the two methods for a number of simulated test cases and studies the influence on the performance of the models when changing selected parameters. Finally, the performance of the methods is compared in a simple measurement setup.

\section{9:40}

3aNSc6. Precise localisation of noise source using statistically optimal beamforming and post -processing deconvolution. Jean-Claude Pascal (ENSIM - LAUM, Université du Maine, rue Aristote, 72085 Le Mans, France, Jean-Claude.Pascal@univ-lemans.fr), Jing-Fang Li (Visual VibroAcoustics, 51 rue d'Alger, 72000 Le Mans, France, jingfang.li@visualvibroacoustics.com), Xavier Carniel (Centre Technique des Industries Mécaniques (CETIM), 52, avenue Félix Louat, 60304 Senlis, France, Xavier.Carniel@cetim.fr), Axel Hass (52, avenue Félix Louat, 60304 Senlis, France, Axel.Haas@cetim.fr), Laurent Bollade (DYVA - DYnamique Vibration \& Acoustique, 10 Rue Maryse Bastié, 69008 Lyon, France, laurent.bollade@dyva.fr)

Conventional data-independent beamforming with microphone array is widely used to locate sound sources and build acoustic model of equipments, engines and vehicles. The particularities of this technique are the use of a relatively few number of microphones and a simple signal processing. So, this technique is fast and easy to use. Other approaches using experimental data for computing the steering vector have been proposed to increase the resolution, for example, minimum variance method, and high-resolution methods. However these methods are often delicate to put in use in acoustic engineering where they are often reproached for lack of robustness in perturbed acoustic fields. Whereas, the most used methods nowadays are those by which the determination of the steering vector is data-independent. Among those methods we consider a method using a Statistically Optimal Array Processing (SOAP) completed by techniques of post-processing deconvolution, by which the resolution of the conventional beamforming is considerably increased, particularly at low frequencies. Some relevant indicators evaluate the performance of the proposed method by using perturbed data and by comparing between conventional and high-resolution beamformers. Examples of industrial measurements in a multi-sources environment demonstrate the practical interest of this technique.

\section{0:00-10:20 Break}

\section{$10: 20$}

3aNSc7. Directional Recording and Analysis of Sounds from Musical Instruments. Franz Zotter (Institute of Electronic Music and Acoustics, Inffeldgasse 10 / 3, 8010 Graz, Austria, zotter@iem.at)

The sound of musical instruments doesn't radiate omni directionally into the sound field. Moreover, each component of the sound (e.g. a partial) can have its own directivity pattern which, in turn, may also vary over time. In general, the radiated sound in free space will have a different timbre at different locations around the sound source. In many cases the description of a particular radiation pattern contour may not be valid for other instruments, tones, playing techniques or even the same musical gesture on the same instrument. Consequently, this work focuses on the synchronous capture and analysis of musical sound sources with a spherical microphone array. 
In order to analyze the directivity in its very detail, a spatial model for radiated sound is suggested. Assuming coherent signals, a common signal decomposition is applicable. The relations between the signal components yield the desired directivity patterns. Spherical harmonics interpolation between the discrete measurement points allows for the estimation of the radiation in a continuous angular space.

\section{Contributed Paper}

\section{0:40}

3aNSc8. Improving the localisation of sources based on shaped arrays with a reduced number of microphones. Lucille L. Lamotte (MicrodB, 7b allée Claude Debussy, 69130 Ecully, France, 11@microdb.fr), Quentin Leclere (Laboratoire Vibrations Acoustique - INSA Lyon, 25 bis avenue Jean Capelle, Bâtiment Saint-Exupéry, F-69621 Villeurbanne cedex, France, quentin.leclere@insa-lyon.fr)

The capacity of hardware today allows carrying out noise sources locali- sation without moving the microphone array at a limited financial and time cost. The single shot measurement processed with beamforming is comfortable for the user. But the quality of the results limits the interest of such systems: bad resolution, low dynamics and no quantitative levels. This article proposes complementary methods to beamforming to improve the results based on the same single measurement. First a near field beamforming algorithm is developed improving the resolution, and then an inverse method is applied based on the transfer function between the sources and the acoustic pressure at microphone positions or on the hologram in order to "clean" the localisation map and give quantitative results.

\section{Invited Paper}

\section{1:00}

3aNSc9. Array based measurement of radiated and absorbed sound intensity components. Jørgen Hald (Brüel \& Kjær Sound \& Vibration Measurement A/S, Skodsborgvej 307, DK-2850 Nærum, Denmark, JHALD@bksv.com), Jakob Mørkholt (Brüel \& Kjær Sound \& Vibration Measurement A/S, Skodsborgvej 307, DK-2850 Nærum, Denmark, jmorkholt@ bksv.com), Pierre Hardy (Dassault Aviation, 80 quai marcel Dassault - Cédex 300, 92552 Saint Cloud, Cédex, France, Pierre.Hardy@dassault-aviation.com), Dominique Trentin (Dassault Aviation, 80 quai marcel Dassault - Cédex 300, 92552 Saint Cloud, Cédex, France, Dominique.Trentin @dassault-aviation.com), Martin Bach-Andersen (Ødegaard \& Danneskiold-Samsøe A/S, Titangade 15, DK-2200 København Ø, Denmark, martin.bach-andersen@lr-ods.com), Graeme Keith (Ødegaard \& Danneskiold-Samsøe A/S, Titangade 15, DK-2200 København Ø, Denmark, Graeme.Keith@1r-ods.com)

Calibration of energy flow models for cabin environments requires tools able to measure the energy flow between the sub-systems of the models. Here we shall specifically consider the energy flow between acoustic energy in the cabin and vibration energy in the panels. Sound intensity measurement, for example with a two-microphone intensity probe, can provide the total sound intensity at some distance from panels. It cannot resolve, how much of the intensity is due to radiation from the surface and how much is due to absorption of energy from the sound field. And it cannot provide the information right on the panel surface, leading to a degraded resolution. The ability to distinguish between radiated and absorbed intensity is important for energy flow modeling, and for identification of the areas where noise energy enters the cabin. The present paper describes some work done to measure the radiated and absorbed intensity components by the use of a double layer microphone array. Two measurements are performed: A measurement of surface absorption coefficient and an operational measurement. Non-locally reacting surfaces represent a particular challenge, the handling of which will be discussed. Results from both loudspeaker measurements and in-flight measurements will be presented.

\section{Contributed Papers}

\section{1:20}

3aNSc10. Cyclic Sound Intensity and Source separation from NAH measurements on a Diesel engine. Brice Lafon (Renault, 67 rue des Bons Raisins, CTR A03 3 37, 92500 Rueil Malmaison, France, brice.lafon @ renault.com), Jerome Antoni (Université de Technologie de Compiègne, Centre de Recherche de Royallieu, rue Personne de Roberval, 60200 Compiègne, France, jerome.antoni@utc.fr)

Noise source separation and acoustic imaging are two research fields of interest in car industry. For this, RENAULT (French car manufacturer) and the University of Technology of Compiègne (UTC, France) are conducting an original study aiming to couple these fields to solve in an efficient way engine NVH problems. Previously, in the first part of this study, combustion noise separation on Diesel engine radiation was implemented from classical sound intensity measurements. The separation, based on Wiener filtering, was performed by taking advantage from the cyclostationary property of engine signals. The second part, presented here, aims to overcome the main shortcoming of the method: time-consuming measurements. Our method is then applied to Near-field Acoustic Holography that is faster and more adapted to an industrial context. Source separation is implemented to complete global radiation maps by specific-source radiation maps such as combustion and other sources radiating in low frequencies since NAH performs rather in low frequencies. Furthermore, the exploitation of cyclostationarity enables to define a cyclic sound intensity (an instantaneous intensity estimated during an average engine cycle). Then, radiation movies can be set to allow a precise location of a specific radiation emergence in space, in frequency and in crankshaft angle.

\section{1:40}

3aNSc11. Tire radiation in vehicle environment: A review of some source identification methods. Christophe Picard (PSA Peugeot Citroën, Route de Gisy, 78943 Vélizy-Villacoublay Cedex, France, christophe .picard3@mpsa.com), Matthieu Fiack (Segula Technologies for PCA, Route de Gisy, 78943 Vélizy-Villacoublay Cedex, France, matthieu .fiack1@ext.mpsa.com), Olivier Tanneau (Altran AIT for PCA, Route de Gisy, 78943 Vélizy-Villacoublay Cedex, France, tanneau77@hotmail .com), Olivier Sauvage (PSA Peugeot Citroën, Route de Gisy, 78943 Vélizy-Villacoublay Cedex, France, olivier.sauvage@mpsa.com), Laurent Gagliardini (PSA Peugeot Citroën, Route de Gisy, 78943 VélizyVillacoublay Cedex, France, laurent.gagliardini@mpsa.com)

The REBECA research project aims at defining new architectural concepts and reduction strategies of vehicle external noise emission regarding the international pass-by noise certification standard (ISO 362). The first task of the project is to qualify and quantify the major contributor of the external emission, the tires/road surface source. To this end, a 3D array composed of 164 microphones has been set up in the near and far field around vehicle. Measurements have been performed for several operational conditions with the use of a chassis dyno in a semi-anechoic chamber. A review and a comparison of some source identification methods has been led including experimental FRFs based on inverse techniques (Nelson), conventional and optimized beamforming approaches (Elias, Dougherty, Brooks and Humphreys, Ravetta) and inverse BEM method (Hamdi, Omrani). This 
study permits to identify the more appropriate microphone array technique with respect to source identification robustness, power level and directivity radiation reconstruction in the far field.

12:00

3aNSc12. Large array optimization using a genetic algorithm and application to wide band moving sources imaging. Sébastien Debert (PSA-Peugeot-Citroën, Route de Gizy, CC : VV013 - Bât. 14, F-78943 Vélizy-Villacoublay Cedex, France, sebastien.debert@mpsa .com), François Ollivier (UPMC Univ Paris 06, CNRS UMR 7190 Institut Jean Le Rond D'Alembert, 2 Place de la Gare de Ceinture, 78210 Saint Cyr l'Ecole, France, frol@ccr.jussieu.fr)

In order to design a microphone array able to follow moving wide band acoustic sources, and improve the quality of the images obtained with a classical beam forming technique, numerical simulations were carried out. The various steps of the study are presented here. Searching the best design for a large microphone array, a simple genetic algorithm was developed. Considering a constant outer dimension of the array and technological constraints in order to design an adaptive array of 60 microphones, the algorithm selects among a large set several combinations of their positions. The functional being optimized is the directivity pattern, in terms of side lobes level. The genetic algorithm always converges to solutions comparable to a randomized set of microphones. Besides, taking advantage of symmetry properties of the array, an improvement of the speed of beamforming calculation was introduced. The optimum array being chosen, wide band moving sources were simulated and images were processed. In order to provide beam formed images with a constant resolution over the band a homoteticbalanced array of 120 microphones was assembled. Finally the dynamic of the images was significantly improved by performing a non-coherent image synthesis technique.

\section{2:20}

3aNSc13. Non stationary tire-road noise analysis using large array beam forming in the time and frequency domains. François Ollivier (UPMC Univ Paris 06, CNRS UMR 7190 Institut Jean Le Rond D’Alembert, 2 Place de la Gare de Ceinture, 78210 Saint Cyr l'Ecole, France, frol@ccr.jussieu.fr)

In the framework of the REBECA Project founded by the ADEME, one seeks to provide a description of the tire-road noise source from the exterior of the vehicle perspective. Far field acoustic imaging techniques were proposed to identify the sources. Therefore an optimised array of 120 microphones, $4 \mathrm{~m}$ in diameter, was modelled and built. Measurements of the noise produced by a car mounted on a roll were performed in a semi anechoïc room. The front car axle was driven at various constant speeds and to rising speeds as well. Beam formed images and point signals have been postprocessed with a $125 \mathrm{kHz}$ sampling rate in order to derive space and time analysis of the pressure field in the vicinity of the tire-roll contact point. The high rate movies processed clearly exhibit periodic phenomena generated around the contact point, while the tire structure borne source appears to be insignificant. Results of frequency and order analysis are also presented in this attempt to provide an interpretation of the tire/road contact noise in terms of source models.

\title{
Session 3aNSd
}

\section{Noise and EURONOISE: Tire-Road Noise from the Road Perspective I}

\author{
Paul Donavan, Cochair \\ Illingworth \& Rodkin, Inc., 505 Petaluma Blvd. South, Petaluma, CA 94952, USA \\ Fabienne Anfosso-Ledee, Cochair \\ Laboratoire Central des Ponts et Chaussées, BP 4129, Bouguenais Cedex, 44341, France
}

Invited Papers

\begin{abstract}
8:00
3aNSd1. Tyre/road noise prediction: A comparison between the SPERoN and HyRoNE models - Part 1. Thomas Beckenbauer (Müller-BBM GmbH, Robert-Koch-Straße 11, 82152 Planegg, Germany, T.Beckenbauer@MuellerBBM.de), Philippe Klein (INRETS, 25 av. F. Mitterrand, case 24, 69675 Bron, France, philippe.klein@inrets.fr), Jean-François Hamet (INRETS, 25 av. F. Mitterrand, case 24, 69675 Bron, France, hamet@inrets.fr), Wolfgang Kropp (Chalmers University of Technology, Division of Applied Acoustics, SE41296 Gothenburg, Sweden, wolfgang.kropp@chalmers.se)

The SPERoN and HyRoNE models predict the pass-by tyre/road noise of a passenger car from intrinsic characteristics of the road surface. Both models are hybrid: they combine statistical laws with physical models. With a computing time of a few minutes (very quick compared to full physical models), they provide operational tools for tyre/road noise prediction. Particular fields of interest are road surface optimisation with respect to noise at the laboratory scale, conformity of production of a new surface and acoustic monitoring of roads. They are now implemented as user-friendly stand-alone applications. The presentation will address the principles of the models, their performances and their respective main fields of application. Part 1 will address the principles of the models and their respective fields of application.
\end{abstract}


3aNSd2. Tyre/road noise prediction: A comparison between the SPERoN and HyRoNE models - Part 2. Philippe Klein (INRETS, 25 av. F. Mitterrand, case 24, 69675 Bron, France, philippe.klein@inrets.fr), Thomas Beckenbauer (Müller-BBM GmbH, Robert-Koch-Straße 11, 82152 Planegg, Germany, T.Beckenbauer@MuellerBBM.de), Jean-François Hamet (INRETS, 25 av. F. Mitterrand, case 24, 69675 Bron, France, hamet@inrets.fr), Wolfgang Kropp (Chalmers University of Technology, Division of Applied Acoustics, SE-41296 Gothenburg, Sweden, wolfgang.kropp@chalmers.se)

The SPERoN and HyRoNE models predict the pass-by tyre/road noise of a passenger car from intrinsic characteristics of the road surface. Both models are hybrid: they combine statistical laws with physical models. With a computing time of a few minutes (very quick compared to full physical models), they provide operational tools for tyre/road noise prediction. Particular fields of interest are road surface optimisation with respect to noise at the laboratory scale, conformity of production of a new surface and acoustic monitoring of roads. They are now implemented as user-friendly stand-alone applications. The presentation will address the principles of the models, their performances and their respective main fields of application. Part 2 will address the performances of the models.

\section{8:40}

3aNSd3. Experimental study of dynamical contact forces for tyre/road noise application. Julien Cesbron (Université d'Evry Val d'Essonne, Laboratoire de Mécanique d'Evry, EA3332, 40, rue du Pelvoux, 91020 Evry Cedex, France, cesbron.julien@neuf.fr), Fabienne Anfosso-Ledee (Laboratoire Central des Ponts et Chaussées, BP 4129, 44341 Bouguenais Cedex, France, fabienne.anfosso@lcpc.fr), Denis Duhamel (ENPC, UR Navier, 6 et 8 Avenue Blaise Pascal, Cité Descartes, Champs sur Marne, 77455 Marne la Vallée, France, duhamel@lami.enpc.fr), Hai Ping Yin (ENPC, UR Navier, 6 et 8 Avenue Blaise Pascal, Cité Descartes, Champs sur Marne, 77455 Marne la Vallée, France, yin@lami.enpc.fr), Donatien Le Houedec (Ecole Centrale de Nantes, GeM, 1 rue de la Noe, BP 92101, 44321 Nantes Cedex 3, France, Donatien.Lehouedec@ec-nantes.fr)

This paper deals with the experimental study of tyre/road contact forces in rolling conditions for tyre/road noise investigation. In situ measurements of contact forces and CPX noise levels were carried out for a slick tyre rolling on six different road surfaces at rolling speeds between $30 \mathrm{~km} / \mathrm{h}$ and $50 \mathrm{~km} / \mathrm{h}$. Contact stresses were measured at a sampling frequency around $10000 \mathrm{~Hz}$ using a single array of digital pressure sensitive cells both in the transverse and in the longitudinal directions. The contact areas obtained during rolling were smaller than the one measured in statics. This could be due to an influence of the viscoelastic behavior of the rubber of the tyre tread during rolling. Additionally the global energy of the longitudinal contact forces at different speeds were in the same order for the six road surfaces, while their spectrums at different speeds were quite different. This may be explained by a spectral influence of the vibration of the tyre during rolling. Finally, the levels of longitudinal contact forces and CPX noise measured at $30 \mathrm{~km} / \mathrm{h}$ were correlated. A high positive correlation was found around $800 \mathrm{~Hz}$, which frequency could be directly linked to the road texture.

\section{9:00}

3aNSd4. Optimized thin layers for urban roads. Sigurd N. Thomsen (Danish Road Institute/Road Directorate, Guldalderen 12, 2640 Hedehusene, Denmark, snt@vd.dk), Hans Bendtsen (Danish Road Institute/Road Directorate, Guldalderen 12, 2640 Hedehusene, Denmark, hbe@ vd.dk), Bent Andersen (Danish Road Institute/Road Directorate, Guldalderen 12, 2640 Hedehusene, Denmark, bea@vd.dk)

In the EU project SILENCE work has been carried out to optimize the noise reduction of different types of pavements for urban roads. The Danish Road Institute (DRI) participated in this work together with the Swedish National Road and Transport Research Institute (VTI) and BAST in Germany. Joint international development work has been carried out including laboratory experiments. The objective was to find pavements with promising noise reduction. It was decided to carry out a full scale field testing in some countries. DRI has in cooperation with the municipality of Copenhagen and the Colas road construction company tested series of SMA pavements and open graded pavements, optimized for noise reduction by using small aggregate size of 4 to $6 \mathrm{~mm}$ and by constructing a relatively high builtin air void. Eight different pavements were constructed in June 2007 on Kastrupvej in Copenhagen. DRI has conducted SPB and CPX noise measurements. An initial noise reduction for passenger cars of $4.3 \mathrm{~dB}$ in relation to a DAC 11 reference pavement has been achieved. It is planned to continue the measurements in the coming years in order to be able to analyse the long time acoustical and structural performance of these test pavements.

9:20

3aNSd5. Traffic noise levels after replacing the top of two-layer porous asphalt at Øster Søgade. Jørgen Kragh (Danish Road Institute/Road Directorate, Guldalderen 12, 2640 Hedehusene, Denmark, kragh@vd.dk), Hans Bendtsen (Danish Road Institute/Road Directorate, Guldalderen 12, 2640 Hedehusene, Denmark, hbe@vd.dk)

In 1999 three different types of two-layer porous asphalt pavement with $5 \mathrm{~mm}$ or $8 \mathrm{~mm}$ maximum aggregate in the top layer were built in a city street in Copenhagen. A section with dense asphalt concrete was built as a reference. The pavements have been monitored since then. The initial noise reduction of $6-7 \mathrm{~dB}$ compared with the noise level at the reference section has vanished over the years due to gradual clogging of the porosities in the pavement. After 8 years in service the drainage asphalt in the spring of 2007 was worn and ravelling had begun. The municipality of Copenhagen decided to have the top layer milled away and replaced by new porous asphalt with $8 \mathrm{~mm}$ maximum aggregate. This type had proven most durable, acoustically and structurally. Noise measurements were carried out in 2007 as part of the SILENCE project, both before and after replacing the top layer. The measurements showed that at two of the test sections most of the initial noise reduction was regained while this was not the case at the one test section that was thinnest (a total of $55 \mathrm{~mm}$ while the other were $70 \mathrm{~mm}$ and $90 \mathrm{~mm}$, respectively). 


\section{Contributed Papers}

\section{9:40}

3aNSd6. The effect of grinding and grooving on the noise generation of Portland Cement Concrete pavement. Tanya Wulf (Purdue Univ., 140 Martin Jischke Dr., Herrick Lab., West Lafayette, IN 47907, USA, twulf@purdue.edu), Tyler Dare (Purdue Univ., 140 Martin Jischke Dr., Herrick Lab., West Lafayette, IN 47907, USA, tdare@purdue.edu), Robert Bernhard (Purdue Univ., 140 Martin Jischke Dr., Herrick Lab., West Lafayette, IN 47907, USA, rbernhar@nd.edu)

In this investigation, studies have been done to understand the effects of various grinding and grooving parameters to investigate their effect on noise generation at the tire-pavement interface. Grinding uses diamond-infused blades that are closely-spaced such that the fins between the blade tracks break off exposing an entirely new surface. For grooving, the blades are more widely spaced such that the fins do not break off and the surface texture remains largely unchanged except for grooves that are used for moisture control. Both procedures, used independently or in combination, have an effect on the noise produced by the tire-pavement interaction. Variation of grinding parameters was shown to have as much as a $3 \mathrm{~dB}$ effect on noise generation. Variation in grooving parameters has a secondary effect, which allows grooves to be added to texture without overall effect on overall noise. This paper will illustrate the effects on noise of the different parameters, such as grinding depth, blade width, and blade spacing, for grinding and grooving.

\section{0:00}

3aNSd7. Monitoring 20 Silent Roads Over 10 Years In The Municipality Of Groningen. Wim Van Keulen (Vankeulen Advies BV, De Savornin Lohmanlaan 68, 5252 AJ Vlijmen, Netherlands, info @ vankeulenadvies.nl)

The municipality of Groningen in the northern part of the Netherlands has a wide experience with the application of silent roads. This experience covers a period of about 20 tracks with 2-layered porous asphalt and various types of thin layers. These tracks have been and are monitored regularly. The obtained data from 10 years monitoring offer a vast amount of valuable information on noise reduction, durability, maintenance, costs, contracts and policy. The municipality of Groningen in the northern part of the Netherlands has a wide experience with the application of silent roads. This experience covers a period of about 20 tracks with 2-layered porous asphalt and various types of thin layers. These tracks have been and are monitored regularly. The obtained data from 10 years monitoring offer a vast amount of valuable information on noise reduction, durability, maintenance, costs, contracts and policy.

\section{0:20-10:40 Break}

\section{0:40}

3aNSd8. A quiet poroelastic road surface manufactured in a normal asphalt mixing plant. Nils-Åke Nilsson (Acoustic Control AB, Tumstocksvägen 1, SE-187 66 Taeby, Sweden, na.nilsson@acoustic.se), Nils Ulmgren (NCC Roads Sweden AB, R\&D Center, Bryggervägen 13, SE194 36 Upplands Väsby, Sweden, nils.ulmgren@ncc.se), Ake Sandin (City of Goteborg, Traffic and Public Transport Authority, PO Box 2403, 40316 Goteborg, Sweden, ake.sandin@ trafikkontoret.goteborg.se)

A poroelastic road surface for low tire/road noise has been developed. It is manufactured using conventional asphalt mixing plants and conventional paving machines. Crumb rubber was added to the mix for attaining the desired elastic characteristics. The crumb rubber was subject to a long term pre-treatment before feeding it to the plant mixer. This ensures both long term durability and excellent adhesion to the binder. The crumb rubber grain size and amount in the mix has been carefully tuned to the aggregate grain size. By the pre-treatment of the crumb rubber the crumb rubber amount can be freely selected since it is not locked to the amount of binder (as for the hot bitumen pre-mixer technique) Single layer poroelastic road surface sections has been paved in Gothenburg. The reduction of noise relative to a SMA16 was found to be 4-6 $\mathrm{dB}(\mathrm{A})$ depending on the vehicle speed and amount of crumb rubber. The surface is almost unaffected by wear after 6 months winter traffic with studded tyres. Tuning the recipe for further increased reduction of the tire/road noise continues. Fundings from European Commission (project Quiet City Transport (QCITY)) is acknowledged.

\section{Invited Paper}

\section{1:00}

3aNSd9. Evaluation of the ASTM Standard Reference Test Tire for purposes of standardized measurement of on-bound tire/pavement noise. Paul Donavan (Illingworth \& Rodkin, Inc., 505 Petaluma Blvd. South, Petaluma, CA 94952, USA, pdonavan @illingworthrodkin.com)

Currently in the US, efforts are underway to develop standard methods for on-board sound intensity (OBSI) measurement of tire/pavement noise. Up until recently, the default standard tire was the Goodyear Aquatred 3 tire originally selected due to its apparent similarity to Tire A specified in the ISO CPX procedure. Because of longer-term availability, the ASTM Standard Reference Test Tire (SRTT) is the primary candidate for replacement of the Aquatred 3. Issues of concern for the SRTT include tire-to-tire variation, the relation of the SRTT to previously used reference tire, and the "break-in" period required for stable test tires. To address tire-to-tire variability, six SRTT's were tested on variety of asphalt (AC) and Portland cement concrete (PCC) surfaces. These included four new tires and two that had been in use for some time. Two of the new tires were retested with increasing use to examine any break-in period effect. For comparison, the older Aquatred 3 was also tested on these same surfaces using both OBSI and controlled pass-by measurements. The results of these measurements are presented along with their implication to for reference tire selection.

\section{Contributed Papers}

\section{1:20}

3aNSd10. Evaluation of effects of pavement characteristics on the OBSI levels using Principal Components Regression. Aybike Ongel (Kultur University, Ataköy Kampüsü Bakırköy, 34156 Istanbul, Turkey, aybikeongel@gmail.com), Erwin Kohler (Dynatest, University of California, Davis, CA 95616, USA, ekohler@dynatest.com), John Harvey (University of California, Civil and Environmental Engineering, Davis, CA 95616, USA, jtharvey@ucdavis.edu)

Tire/pavement noise is a major contributor to traffic noise at highway speeds. Tire/pavement noise is affected by different pavement properties. A study conducted in California measured the noise levels of different mix types and the mix characteristics affecting noise levels. In this study, tire/pavement noise was measured using the on-board sound intensity (OBSI) method. Data was collected on four different types of pavement mixes: conventional open graded asphalt concrete (OGAC), rubberized asphalt concrete that are open graded (RAC-O), rubberized asphalt concrete that are gap graded (RAC-G), and dense graded asphalt concrete mixes (DGAC). A total of 72 field pavement sections were included in the study, all of which were less than 8 years old at the time of the measurements. This paper evaluates the pavement characteristics affecting noise levels using principal components regression. This technique was used due to the multicollinearity found among the variables. Two principal components were extracted from 
the measured parameters such as air void content, gradation properties, pavement roughness, age, and pavement surface condition.

\section{$11: 40$}

3aNSd11. Austrian experience with the backing board method for statistical pass-by measurements. Marco Conter (Arsenal Research, Giefinggasse 2, 1210 Vienna, Austria, marco.conter@arsenal.ac .at), Manfred Haider (Arsenal Research, Giefinggasse 2, 1210 Vienna, Austria, manfred.haider@arsenal.ac.at)

Traffic noise is one of the major environmental concerns within the countries of the European Union. 80 to $90 \%$ of the traffic noise pollution is generated by road traffic. The major part of the noise emitted by vehicles on roads in the mid- to high-speed range is nowadays due to tyre/road noise. It is generated by the interaction between tyre and road surface, and therefore the measurement of the typical noise emission is essential for the classification of pavements with regard to noise. The SPB method (Statistical PassBy, ISO 11819-1) is nowadays the most widely used method to characterise road surfaces, but it is difficult to apply especially in the presence of roadside noise barriers. The backing board method, further developed within the EU Project SILENCE in the past years, offers a possibility to measure and to characterise surfaces also close to reflective objects and in urban environments. This paper describes the first Austrian experimental experience with the application of this variant of the SPB method.

\section{2:00}

3aNSd12. Acoustical characterisation and life-cycle of porous road surfaces. Beate Altreuther (Mueller-BBM GmbH, Robert-Koch-Str. 11, 82152 Planegg, Germany, beate.altreuther@muellerbbm.de), Wolfram Bartolomaeus (Bundesanstalt fuer Strassenwesen, Bruederstr. 53, 51427 Bergisch Gladbach, Germany, bartolomaeus@bast.de)

The German Federal Highway Research Institute (Bundesanstalt für Straßenwesen, BASt) is planning to develop an assessment procedure to characterize the acoustical properties of porous road surfaces. Final objective is to specify the life-cycle of these pavements with respect to their acoustical performance based on civil engineering properties. In 2007 comprehensive measurements of the acoustically relevant parameters absorption coefficient, air flow resistance and surface texture have been performed on a multitude of bavarian motorways with porous asphalt pavements of different types of construction and different ages. Additional measurements of the near-field rolling noise $(\mathrm{CPX})$ as well as measurements of the statistical pass-by-noise (SPB) have been performed. From precedent projects for the sections under investigation there is an extensive database covering details on asphalt mixtures as well as the results of drill core investigations concerning void content, binder content etc. The results of the present investigation allow to draw new conclusions with respect to acoustical performance and durability of porous asphalt pavements. We gratefully acknowledge the project funding by the German Federal Ministry of Transport, Building and Urban Affairs (project No. FE 02.276/2006/LRB).

\section{2:20}

3aNSd13. DEUFRABASE: A German-French acoustic database on road pavements. Michel C. Berengier (Laboratoire Central des Ponts et Chaussées, Centre de Nantes - Route de Bouaye, BP 4129, 44341 Bouguenais cedex, France, Michel.Berengier@lcpc.fr), Bettina
Droste(Bundesanstalt für Strassenwesen, Brüderstrasse 53, 51427 Bergisch Gladbach, Germany, droste@bast.de), Benoit Gauvreau (Laboratoire Central des Ponts et Chaussées, Centre de Nantes - Route de Bouaye, BP 4129, 44341 Bouguenais cedex, France, benoit.gauvreau@lcpc.fr), Denis Duhamel (ENPC, UR Navier, 6 et 8 Avenue Blaise Pascal, Cité Descartes, Champs sur Marne, 77455 Marne la Vallée, France, duhamel@lami .enpc.fr), Markus Auerbach (Bundesanstalt für Strassenwesen, Brüderstrasse 53, 51427 Bergisch Gladbach, Germany, m.auerbach@bast.de)

In the framework of a DEUFRAKO (German/French cooperation) project on the Prediction and Propagation of Rolling Noise (P2RN), it has been proposed to rank the different German and French road pavements with respect to noise for different long distance configurations representative of real road topographies, ground characteristics and meteorological situations. After identification of those typical 28 configurations, the attenuations between a reference point in the near field and several receiving points in the far field have been computed according to the most relevant and adapted modelling methods currently available. The main goal being the accuracy of the prediction and not the computation time, analytical (ray tracing) and numerical (BEM, Parabolic Equation) approaches have been implemented. In the following, a technique permitting to estimate day and night LAeq and Lden has been used to classify the various pavements for the whole configurations. All those results have been gathered in a common database (DEUFRABASE) which will be directly available on the website of the authors' Institutes. The paper deals with the description of the ranking procedures, how the database is implemented on the web and how to use it for road traffic noise prediction.

\section{2:40}

3aNSd14. Road noise characterization by Harmonoise procedures reviewed for the Italian case. Luca Alfinito (University of Pisa - Dept. of Civil Engineering, Via Diotisalvi, 2, 56100 Pisa, Italy, 1.alfinito@arpat .toscana.it), Gaetano Licitra (ARPAT - Dept. Firenze, Via Porpora, 22, 50144 Firenze, Italy, g.licitra@arpat.toscana.it), Mauro Cerchiai (ARPAT Dept. Pisa, Via V. Veneto, 27, 56127 Pisa, Italy, m.cerchiai@arpat.toscana .it), Lorenzo Magni (ARPAT - Dept. Pisa, Via V. Veneto, 27, 56127 Pisa, Italy, 1.magni@arpat.toscana.it), Massimo Losa (University of Pisa - Dept. of Civil Engineering, Via Diotisalvi, 2, 56100 Pisa, Italy, losa@ing.unipi.it)

Several methods have been introduced to describe acoustical properties of road pavements in situ, such as the extended surface technique (Adrienne), the Statistical Pass-By and the Close Proximity methods. In general the aim of these techniques, normed in ISO standards, is to evaluate some indexes that may be representative of noise generation and propagation with respect to tyre-road interaction and surface absorption. In this paper we use results obtained in the LEOPOLDO project, that involves measurements carried out on many sites all over Tuscany Region characterized by typical conditions of Italian roads, to review the state-of-the-art of acoustical parameters in the modelling of road vehicles showed in the HARMONOISE documents. This task has been accomplished by comparing the theoretical results obtained from the HARMONOISE prediction method with the energy levels measured at the microphone locations close to the road borders, in order to apply corrections to the parameters influencing sound generation and propagation. As a consequence, we introduce a new parameter, related to transfer function between source and receiver sound energy levels, which involves directivity and ground reflection effects, obtaining a more suitable characterization of road surface acoustical behaviour. 


\title{
Session 3aNSe
}

\section{Noise, Structural Acoustics and Vibration, Signal Processing in Acoustics, and EURONOISE: Airframe Noise Measurement, Prediction, and Control I}

\author{
Joe W. Posey, Cochair \\ NASA, Langley Research Center, MS 461, Hampton, VA 23681, USA \\ Denis Gely, Cochair \\ Département Simulation Numérique des écoulements et Aéroacoustique (DSNA), Onera, BP72 - 29 avenue de la Division \\ Leclerc, 92320 Chatillon, France
}

Invited Paper

10:00

\begin{abstract}
3aNSe1. Airframe noise prediction. Yueping Guo (Boeing, 5301 Bolsa Ave, Huntington Beach, CA 92647, USA, yueping.guo @boeing.com)

This presentation discusses the prediction of aircraft airframe noise, namely, the noise that is generated by non-propulsion components of aircraft, including the leaning edge slats, the side edges of flaps and the landing gears. An overview will be given on various methodologies, ranging from empirical prediction to more physics-based modeling to full-blown numerical simulation. The advantages of each method will be discussed and compared with each other to reveal the most suitable application domain of each method. Detailed discussions will be given to the physics-based modeling, such as the acoustic analogy, which is ranked in between empirical and numerical methods in various aspects, including accuracy, turn-around time, limitations on geometry and flow conditions, and applicability/ feasibility in practical design applications. Examples will be given to demonstrate the applications of acoustic analogy in both noise prediction and noise reduction treatment design. Critical elements of the method that can and need to be improved to further mature the method will also be identified and discussed, providing potential directions for future research and development.
\end{abstract}

\section{Contributed Paper}

10:20

3aNSe2. ONERA's activities on airframe noise numerical simulation. Eric Manoha (ONERA (French aerospace Center), CFD \& Aeroacoustics Department, BP 72, 29 avenue de la division Leclerc, 92322 Chatillon Cedex, France, eric.manoha@onera.fr)

Airframe noise, or noise generated by flow around the airframe (high lift devices, landing gears and cavities), is a major contributor to the overall noise of large commercial aircraft in the approach phase. For several years, ONERA has been involved in the development of methodologies for the numerical simulation of airframe noise. These hybrid methods generally combine (i) CFD computations for the near field flow, including unsteady CFD techniques such as LES, DES and NLDE, and (ii) acoustic (CAA) propagation techniques in the mid- and far-field. A large range of acoustical methods is available for the propagation of sound waves in uniform or heterogeneous mean flows, either from the family of integral methods (Lighthill, FWH, $\mathrm{BEM}$ ) or in the domain of (non-linear or linearized) Euler equations in perturbation. The key challenges of these hybrid methodologie are (i) the ad-hoc coupling between individual techniques and (ii) the availability of reliable and dedicated experimental database for the validation. The paper presents a survey of ONERA's activities in this field, with applications to (i) single symmetrical airfoil, (ii) high lift wing with deployed slat and flap, (iii) landing gears and (iv) cylindrical cavities.

\section{Invited Papers}

\section{0:40}

3aNSe3. Measurement techniques for airframe noise source identification. Ricardo A. Burdisso (Virginia Tech, Mechanical Engineering, 153 Durham 0238, Blacksburg, VA 24061, USA, rburdiss@vt.edu)

Despite the significant progress achieved over the last three decades, noise pollution generated by aircraft around airports is still an obstacle to the further growth of the aviation industry. The engines and airframe constitute the main sources of the overall aircraft noise. Airframe noise is as important as the engine noise on approach due to the engines operating at low thrust. High-lift devices and landing gears are the dominant airframe noise components. Many efforts have recently been devoted to accurately locate and control the specific components of the airframe noise. Experimental studies on airframe noise include full-scale flight tests, and tests conducted on isolated small-scale models in both hard-walled and anechoic wind tunnels. These experimental efforts on airframe noise have been greatly helped by the advent of the microphone phased array technology. This presentation will focus on microphone phased array measurement techniques, in particular for model scale testing in wind tunnels. Issues such as beamforming algorithms, data corrections, effect of flow and acoustic environment on the measurements (hard-wall vs anechoic tunnels), etc. will be discussed. Illustrative results from experimental measurements of landing gear and high lift devices performed at the Virginia Tech wind tunnel will also be presented. 
3aNSe4. Reduction technologies and prediction of high lift noise. Jan W. Delfs (DLR/Institute of Aerodynamics and Flow Technology, Lilienthalplatz 7, 38108 Braunschweig, Germany, jan.delfs@dlr.de)

In the final approach phase airframe noise represents the ultimate aircraft noise barrier for future aircraft when equipped with quiet UHBR engines. This paper presents some of the main dependencies of high-lift noise sources on flow conditions as derived from extensive noise testing at DLR. Some successful reduction technologies for high-lift noise are discussed in view of their influence on the noise generation mechanisms. The remainder of the paper focusses on the numerical prediction of high-lift noise. The prediction concept as implemented in DLR's PIANO code is discussed. It is based on a two step approach, a) the computation of the time averaged turbulent flow field as a result of a $\mathrm{CFD}(=$ Computational Fluid Dynamics) simulation and $\mathrm{b})$ the highly accurate CAA (=Computational Aeroacoustics) simulation of perturbations about this mean flow. The prediction relies on an advanced stochastic modelling of the turbulence related sources and thus avoids time consuming turbulence simulations. The method represents an accurate yet "low cost" prediction of high-lift noise. This is particularly important for real applications at flight (very high) Reynolds numbers and corresponding design trade-off studies, which typically require the computation of large numbers of configurations. Examples for such kind of applications are given.

\section{Contributed Paper}

$11: 20$

3aNSe5. Acoustic characterization and noise reduction strategies for a general aviation aircraft. Nicola Rusciano (Dept. of Aerospace Engineering - University of Naples, Via Claudio, 21, 80125 Naples, Italy, nruscian@unina.it), Massimo Viscardi (Dept. of Aerospace Engineering University of Naples, Via Claudio, 21, 80125 Naples, Italy, massimo .viscardi@unina.it)

This work concerns with the vibro-acoustic characterization of a general aviation aircraft with the objective of designing solutions to reduce the inside noise level during the flight; first of all a new passive insulation, and then verifying the possibility of an active noise control approach. The acoustic characterization was performed into two different step; in-flight acoustic and vibration measurements during a typical mission of the vehicle were initially measured, to characterize the acoustic levels and spectra elated to the different flight operations. Ground measurement were also performed to characterize the sound insulation properties of the aircraft structure. The next item was the partial re-design of the sound insulation pack for the vehicle by the use of innovative material; the choice of the best technical solution has been performed by the use of numerical prediction models and through experimental tests on modified samples of the aircraft structure. The in-flight acoustic and vibration measurement showed also the presence of dominant low frequencies tonal components related to the BPF's and upper harmonics. This situation suggested the possibility to implement an active noise control approach. All these activities will be herein presented and discussion of the main results will be performed within the paper.

\section{Invited Paper}

\section{1:40}

3aNSe6. On the reduction of flap side edge noise. Florence Hutcheson (NASA Langley Research Center, Hampton, VA 23681, USA, Florence.V.Hutcheson@nasa.gov), Thomas F. Brooks (NASA Langley Research Center, Hampton, VA 23681, USA, thomas.f .brooks@nasa.gov)

Results from flap side edge noise reduction experiments are presented. The test model used was a NACA 632-215 main-element airfoil with a half-span Fowler flap. The flap side edge was flat for the baseline configuration. The effect that small geometric variations of the flap edge have on the noise spectra is shown, and noise measurements from a "continuous mold-line link" (CML) flap configuration are presented. These measurements are compared to those obtained from the baseline flap. The results indicate that a large level of noise reduction is achieved using the CML flap. Finally, Particle Image Velocimetry (PIV) measurements of the flow in the region of a flap side edge are presented for a blowing flap configuration. In this active control approach, air was blown from small slots located along the side edge of the flap to weaken the vortex system that is present in that region of the flap. These measurements are presented to show the effects that the flap tip jets have on the structure of the flap side edge flow, and the potential that this blowing flap configuration has in reducing flap side edge noise.

\section{Contributed Paper}

\section{2:00}

3aNSe7. Landing gear noise assessment on Airbus aircraft. Nicolas Molin (Airbus France, 316 route de Bayonne, Acoustics and Environment Dept EDEA, 31060 Toulouse, France, nicolas.molin@airbus.com)

During the last two decades, airframe noise sources (high lift devices and landing gears) have become noticeable in new aircraft technology, especially during approach. Aircraft manufacturers need prediction tools, in order to include noise modelling at the early stages of aircraft design. Such tools can be developed on the basis of simple formulations. As long as the dominant features are preserved in the simplified problems, the solution is believed to behave correctly in terms of EPNdB. Such a model of landing gear noise prediction was developed in the late 1990s. It is based on the decomposition of a landing gear into different noise sources: the primary structure (massive elements) and the secondary structure (wire, pipes, œ), whose noise is estimated with existing classical source noise model fed with empirical constants based on wind tunnel test results. Airbus implemented this model into its total aircraft noise program and validated it with dedicated flight tests. As the model takes into account all main components with their geometry, it is well adapted to predict the impact of add-on treatment such as fairings on the gear or the impact of an advanced low noise gear with simplified geometry. 


\title{
Invited Paper
}

$12: 20$

3aNSe8. A design-oriented approach to landing gear noise prediction. Philip J. Morris (Penn State University, 233C Hammond Building, University Park, PA 16802, USA, pjm@psu.edu), Kenneth S. Brentner (Penn State University, 233C Hammond Building, University Park, PA 16802, USA, ksb16@psu.edu), Leonard V. Lopes (Penn State University, 233C Hammond Building, University Park, PA 16802, USA, 1vl105@psu.edu)

Landing gear noise is one of the most important sources of airframe noise. But it is the most difficult to predict. The difficulty is that the landing gear is made up of components of substantially different scales. The small-scale features such as tubes and hoses are known to contribute to the landing gear noise, especially in the mid to high frequency range. Existing semi-empirical models are only reliable for the range of landing gear geometries for which test data are available. The present paper describes a component-based landing gear noise prediction model. The method uses an application of simple, fast, and scalable models called acoustic elements that are used to represent the complex landing gear geometry with a high level of geometric detail. Extensions to an earlier model developed by the authors is described, including the replacement of the original time domain approach with a frequency domain method. The local mean flow is also taken into account through the use of low-fidelity computational fluid dynamics simulations. Estimates of the effects of acoustic shielding are also described. Comparisons are made with available experimental data to demonstrate the capabilities of the method. Future improvements are also described.

\section{Session 3aNSf}

\section{Noise and EURONOISE: Soundscape in the Heritage of Urban and Natural Areas I}

\author{
Bennett Brooks, Cochair \\ Brooks Acoust. Corp., 27 Hartford Turnpike, Vernon, CT 06066, USA \\ Giovanni Brambilla, Cochair \\ CNR Institute of Acoustics, Via del Fosso del Cavaliere 100, Rome, 00133, Italy \\ Invited Papers \\ 10:40
}

3aNSf1. Modeling auditory attention focusing in multisource environments. Bert De Coensel (Ghent University - Department of Information Technology, Sint-Pietersnieuwstraat 41, 9000 Ghent, Belgium, bert.decoensel@intec.ugent.be), Dick Botteldooren (University Ghent - Department Information Technology, Sint-Pietersnieuwstraat 41, 9000 Gent, Belgium, dick.botteldooren@intec .ugent.be)

In this paper, a mathematical submodel for auditory attention focusing is discussed in the framework of our ongoing research towards a unified model for soundscape perception. The submodel implements a balance between top-down focusing, in which higher level cognition guides attention towards expected sources, and bottom-up focusing, in which attention is triggered by the noticing of sound events. Attention elasticity - the ability to switch attention between different environmental sounds - depends on the current context (natural, urban...) as well as on the current activity of the modeled individual. The soundscape perception model is applied to case studies of simulated sound exposure in open area and in environments with a mixture of natural and man-made sounds. A comparison of the simulation results with survey results shows that introducing a submodel for auditory attention focusing into our model for soundscape perception enhances its ability to predict the emergence of annoyance.

\section{1:00}

3aNSf2. SOUND-SCAPE: A framework for characterising positive urban soundscapes. Rebecca Cain (WMG, The University of Warwick, International Manufacturing Centre, CV4 7AL Coventry, UK, R.Cain.1@warwick.ac.uk), Paul Jennings (WMG, The University of Warwick, International Manufacturing Centre, CV4 7AL Coventry, UK, paul.jennings@warwick.ac.uk), Mags Adams (Acoustics Research Centre, University of Salford, Newton Building, Salford, M5 4WT Manchester, UK, M.D.Adams@salford.ac.uk), Neil Bruce (Acoustics Research Centre, University of Salford, Newton Building, Salford, M5 4WT Manchester, UK, N.S.Bruce @ pgr.salford.ac.uk), Angus Carlyle (London College of Communication, University of the Arts, Elephant \& Castle, SE1 6SB London, UK, angus_carlyle@yahoo.co.uk), Peter Cusack (London College of Communication, University of the Arts, Elephant \& Castle, SE1 6SB London, UK, pcusack@btinternet.com), William Davies (Acoustics Research Centre, University of Salford, Newton Building, Salford, M5 4WT Manchester, UK, w.davies@salford.ac.uk), Ken Hume (School of Chemistry, Biological and Health Science, Manchester Metropolitan University, Chester Street, M1 5GD Manchester, UK, K.I.Hume@mmu. ac.uk), Christopher J. Plack (Psychology Department, Lancaster University, LA1 4YF Lancaster, UK, c.plack@lancaster.ac.uk)

People's assessments of urban soundscapes are dependent upon many factors. For example, perceptions of a soundscape may depend on the activity that the listener is doing and their associated listening state at the time. Temporal variations (daily, weekly, seasonal) can also affect perceptions of the soundscape, as can the type and usage of urban space, architectural characteristics and the cultural and historical setting. The context of listening is also a factor in terms of the cognitions that people bring to the listening situation by way 
of memories, preferences, attitudes, values and meaning. In order to inform the planning process with regard to assessing and creating positive urban soundscapes, a framework is under development. The framework attempts to bring together all the influencing factors in urban soundscape assessment in a new and novel way, and considers how the identification of sources and the variety and mix of sources interact to produce a soundscape. The framework presented in this paper forms the basis of multi-disciplinary soundscape research in the EPSRC funded "Positive Soundscapes Project", which seeks to develop a rounded view of human perception of soundscapes by combining methods from several disciplines.

\section{1:20}

3aNSf3. Can urban squares be recognized by means of their Soundscape? Giovanni Brambilla (CNR Institute of Acoustics, Via del Fosso del Cavaliere 100, 00133 Rome, Italy, giovanni.brambilla@idac.rm.cnr.it), Maria Di Gabriele (Built Environment Control Laboratory Ri.A.S., Second University of Naples, Abazia di S. Lorenzo, 81031 Aversa, Italy, maria.digabriele@unina2.it), Luigi Maffei (Built Environment Control Laboratory Ri.A.S., Second University of Naples, Abazia di S. Lorenzo, 81031 Aversa, Italy, luigi.maffei@unina2.it), Patrizio Verardi (CNR Institute of Acoustics, Via del Fosso del Cavaliere 100, 00133 Rome, Italy, patrizio .verardi@idac.rm.cnr.it)

Squares have always played important functions in urban areas, being a reference place in the citizen's life. During last decades, road traffic noise has influenced the perception of the sonic environment in many squares. However, there are squares where historical and typical sound sources are still present. In both cases the sonic environment remains a feature of the square together with other factors, like the visual impact. The present paper deals with the above issue and describes an experiment aimed to study how the soundscape of a square can contribute to recognize the square itself. For this purpose some well known squares in Naples and Rome have been selected to cover a range of different characteristics. In each square binaural recordings and pictures have been taken simultaneously. Then the binaural recordings have been played back by headphone to a group of subjects in laboratory simultaneously to the display of the pictures of all squares on a screen. After listening each sound the subject was asked to choose among the pictures the one showing the square judged to have the sonic environment most appropriate to the sound just heard. The comparison between subjective responses and correct answers are in progress.

\section{1:40}

3aNSf4. Fountains as a natural component of urban soundscape. Catherine Semidor (GRECAU-ENSAPBx, Domaine de Raba, 33400 Talence, France, catherine.semidor@bordeaux.archi.fr), Flora Venot-Gbedji (GRECAU-ENSAPBx, Domaine de Raba, 33400 Talence, France, flora.venot@ bordeaux.archi.fr)

Fountains play an important role in urban landscape as well as being a cultural mark in a city. They also have an environmental function. For example, their effect in the control of the microclimate is well known. The paper deals with the sound effects which big fountains located in large squares have on the urban soundscape of the area. In particular, we deal with how their natural character can change the global perception of the site. Indeed, the representation of water sounds is positively appreciated by most city-dwellers. Depending on their size and shape, they are able to give a quiet or a dynamic character to the site. But in the case of areas with a lot of traffic we ask ourselves what are the interactions with strong background noise level and others sound sources. GRECAU uses the soundwalk method to assess the acoustic environment. The following case studies, Plaza de Cataluna in Barcelona and Piazza Ferrari in Genoa, are two examples of such a situation. Some of the results we give here try to answer the previous questions.

\section{2:00}

3aNSf5. Contemporary soundscape of ancient theatres. Kalliopi Chourmouziadou (School of Architecture, University of Sheffield, Western Bank, S10 2TN Sheffield, UK, pepitahou@hotmail.com), Jian Kang (School of Architecture, University of Sheffield, Western Bank, S10 2TN Sheffield, UK, j.kang@ sheffield.ac.uk)

Ancient drama is performed every summer in ancient theatres in the Mediterranean countries, especially in Greece. In many cases the performance conditions in terms of acoustics are poor, due to the effects of external noise sources. There is often a motorway constructed near an archaeological site, without careful consideration regarding the impact on the overall soundscape. Previous studies have demonstrated the effectiveness of purposely designed scenery on improving the internal acoustic conditions, and this study investigates the contribution of scenery to the improvement of the acoustic environment of ancient theatres in terms of reducing nearby traffic noise. The acoustic simulation for the theatre of Philippi, with scenery design in the form of a background wall in different sizes, suggests that there is a decrease in traffic noise by about $4 \mathrm{~dB}$. Further improvements have also been demonstrated with noise barriers of various heights and lengths. Different levels of traffic flow have been examined. It is expected that the results will be verified by on-site measurements and recordings of summer performances will be presented during the conference.

\section{Contributed Paper}

12:20

3aNSf6. Soundscape and California planning guidelines. Richard B. Rodkin (Illingworth \& Rodkin, Inc., 505 Petaluma Boulevard South, Petaluma, 94952, USA, rrodkin@illingworthrodkin.com), Michael S. Thill (Illingworth \& Rodkin, Inc., 505 Petaluma Boulevard South, Petaluma, 94952, USA, mthill@illingworthrodkin.com)

Community noise has been recognized as a significant environmental concern in California for more than 30 years. All cities and counties are mandated by state law to include a Noise Element as a part of the community's General Plan to provide "a basis for comprehensive local programs to control and abate environmental noise and to protect citizens from excessive exposure." What is, and has been missing from this process, is how the soundscape in a particular setting may affect the compatibility of land uses there. Generalized noise and land use planning guidelines are no longer sufficient. Specific attention must be given to the development of guidelines and policies that address "smart growth" in and around urban and suburban transportation corridors, residential and agricultural interactions, mixed-use developments, industrial-to-residential conversions (e.g., the gentrification of traditionally industrial areas like ports). A new soundscape vocabulary must be introduced to describe the acoustical environments in these settings to facilitate other goals in land use planning. Examples are presented of specific communities where planning goals and traditional noise and land use compatibility guidelines were in conflict and how these were resolved. 


\title{
Session 3aPAa
}

\section{Physical Acoustics: Nonlinear Acoustics of Consolidated Materials and Non Destructive Testing I}

\author{
Jim A. Tencate, Cochair \\ EES-11 (Geophysics) - Los Alamos National Laboratory, MS D443, Los Alamos, NM 87545, USA \\ Koen Van Den Abeele, Cochair \\ K.U.Leuven Campus Kortrijk, E. Sabbelaan 53, Kortrijk, 8500, Belgium
}

\section{Contributed Papers}

\begin{abstract}
8:00
3aPAa1. Three wave mixing test of hyperelasticity in sedimentary rocks. David L. Johnson (Schlumberger-Doll Research, One Hampshire St., Cambridge, MA 02139, USA, johnson10@slb.com), Kenneth Winkler (Schlumberger-Doll Research, One Hampshire St., Cambridge, MA 02139, USA, kwinkler@boston.oilfield.slb.com), Ralph D'Angelo (Schlumberger-Doll Research, One Hampshire St., Cambridge, MA 02139, USA, dangelo2943@boston.oilfield.slb.com)

We report measurements of 3-wave mixing amplitudes on solids whose 3rd order elastic constants have also been measured by means of the elastoacoustic effect. Because attenuation and diffraction are important aspects of our measurement technique we analyze our results using a frequency domain version of the KZK equation, modified to accommodate an arbitrary frequency dependence to the attenuation. We find that the value of $\beta$ so deduced for polymethylmethacrylate (PMMA) agrees quite well with that predicted from the stress-dependent sound speed measurements, establishing that PMMA may be considered a hyperelastic solid, in this context. The $\beta$ values of sedimentary rocks, though they are typically two orders of magnitude larger than e.g. PMMA's, are still a factor 3-10 less than those predicted from the elasto-acoustic effect. Moreover, these samples exhibit significant heterogeneity on a centimeter scale, which heterogeneity is not apparent from a measurement of the position dependent sound speed.
\end{abstract}

\section{8:20}

3aPAa2. Fatigue Damage Monitoring and Remaning Life Assessment using Nonlinear Vibro-Modulation Technique. Dimitri Donskoy (Stevens Institute of Technology, Dabvidson Lab., 711 Hudson Street, Hoboken, NJ 07030, USA, ddonskoy@stevens.edu), Alexander Chudnovsky (Univ. of Illinois at Chicago, Fracture Mechanics \& Material Durability Lab., 842 W. Taylor Styreet, Room 2095 ERF, Chicago, IL 60607, USA, achudnov@uic.edu), Andrei Zagrai (New Mexico Institute of Mining and Technology, Department of Mechanical Engineering, 801 Leroy Pl., Weir Bld., Room 124, Socorro, NM 87801, USA, azagrai@nmt .edu), Edvard Golovin (Univ. of Illinois at Chicago, Fracture Mechanics \& Material Durability Lab., 842 W. Taylor Styreet, Room 2095 ERF, Chicago, IL 60607, USA, egolovin@cs.com), Vinod Agarwala (Naval Air Systems Command, 48110 Shaw Road, Bldg. 2187/Suite 2373, Patuxent River, MD 20670, USA, vinod.agarwala@navy.mil)

Assessment of structural deterioration due to in-service and environmental loads is an essential element in ensuring safety, operability and long life of various structures and structural components. We applied vibro-acoustic modulation technique to monitor material degradation at the micro/meso scale before the onset of the macro-scale fracture. The technique explores nonlinear acoustic interaction of high frequency ultrasound and low frequency structural vibration at the site of the incipient damage. It is shown that micro/meso scale degradation increases the material nonlinearity leading to modulation of the high frequency ultrasonic signal by low frequency vibration, quantified by the nonlinear acoustic Damage Index (DI). Numerous tests with hundreds of test coupons proved high sensitivity of the developed technique to micro/meso scale fatigue damage accumulation. The study further demonstrated that the remaining life of the fatigued material can be predicted using phenomenological damage accumulation rule expressed through measured Damage Index. Work supported by NAVAIR.

\section{8:40}

3aPAa3. Thermal ageing evaluation with nonlinear elastic wave spectroscopy method of carbon fiber reinforced plastic composite plates. Faouzi Guenab (LOMC FRE-3102 CNRS Groupe Ondes Acoustique, Place Robert Schuman BP 4006, 76610 le Havre, France, guenab_faouzi @ yahoo.fr), Hugues Duflo (LOMC FRE CNRS 3102, Université du Havre, Place Robert Schuman, 76610 le Havre, France, hugues.duflo@univ-lehavre .fr)

The excellent and specific properties of composite materials in the various fields such as aeronautics, transport, energy, the nuclear power and civil engineering led to the need of knowing their behaviours in presence of defect (crack, ageing, delamination...). Therefore, the aim of this paper is to study the thermal ageing of the carbon fibre reinforced composite plates, using their nonlinear behaviours. The method used to achieve this work is the NEWS method (Non-linear Elastic Wave Spectroscopy), where two alternatives of this one are used. The first one is SIMONRUS which involves a study of the nonlinear response of a single resonant mode of the plate according to various amplitudes of excitation. In this case, the nonlinear phenomena analysed are resonance frequency shift and damping characteristic of different level ageing of plate. The second one consists in studying the frequential response and the harmonics generated at the multiple of a resonance frequency $f$ (at $f, 2 f, 3 f$...) caused by the ageing. Evolution of non linear effetcts are then correled to ageing duration

\section{9:00}

3aPAa4. Mesoscopic study of the enhancement of the nonlinearity of an ultrasonic wave in inhomogeneous media. André Moura (University of Heidelberg, Institute of Physical Chemistry, AK Hess, Im Neuenheimer Feld 253, 69120 Heidelberg, Germany, Moura@uni-heidelberg.de)

In quantitative nondestructive evaluation of materials it is not still clear why linear elastic waves often exhibit substantial nonlinearity when traveling through inhomogeneous medium, whereas this effect exists but remains minor in most homogeneous media. A current dominant thesis claims that when damaged, intact brittle materials become nonlinear mesoscopic elastic materials. Although this model is consistent with macroscopic observations in the framework of continuum mechanics, it eludes, however, a fundamental aspect, i.e., the detailed nature of the wave/microstructure interaction like scattering and transmission through microcrack. To construct a unified theory, from the mesoscopic to the macroscopic scale, that could take account altogether such so different mesoscopic elastodynamic effects is our objective. Toward this aim, many aspects of the wave/slit interaction are thoroughly analyzed among the clapping effect and nonlinear scattering, and experimental schemes are scheduled. At the current state of our knowledge, a first general postulate is claimed. 
3aPAa5. A phase-pegistration acoustic method for nondestructive testing of porous materials. Valery M. Tsaplev (North-West State Technical University, 5, Millionnaya Street, 191186 Saint-Petersburg, Russian Federation, valery@lek.ru)

The well-known method of complex vibrator is modified for the purpose of meas-urements of complex elastic moduli (Young's and shear moduli) and corresponding values of internal friction, that change under the influence of different external factors. These external factors are: mechanical uniaxial compressive or tension stresses, temperature, electric and/or magnetic fields, etc. Different variants of phase-control methods in the selfexcitation system of the complex vibrator are considered depending on the connection of the active piezoelec-tric bars: active external control or adaptive self-control at prescribed level of the in-put excitation level. The system contains the recording device to record quick changes of elastic and unelastic parameters of the test specimen. The system also contains the termostatic switch with the active control device to provide the specimen temperature control at prescribed algorithm. The method is used for the nondestructive testing of the acoustic properties of different materials under the influence of the stepwise applied mechanical stress.

\section{0:00}

3aPAa6. Nonlinear-nonequilibrium wave propagation in sandstones. Donatella Pasqualini (Los Alamos National Laboratory, POBox 1663, Los Alamos, NM 87545, USA, dondy@lanl.gov), Jim A. Tencate (EES-11 (Geophysics) - Los Alamos National Laboratory, MS D443, Los Alamos, NM 87545, USA, tencate@lanl.gov), Salman Habib (Los Alamos National Laboratory, POBox 1663, Los Alamos, NM 87545, USA, habib@lanl.gov)

The transition from linear to nonlinear-nonequilibrium dynamical elasticity in rocks is of considerable interest in seismic wave propagation as well as in understanding the basic dynamical processes in consolidated granular materials. We have carried out a careful experimental investigation of this transition for Berea and Fontainebleau sandstones. These experiments have showed the existence of two strain regimes. At low strain (10-9 up to 10-7) the rocks behave elastically as classical (Landau-Lifshitz theory) nonlinear materials. At higher strains memory effects due to a driven nonequilibrium state complicate the characterization of the nonlinear behavior. The understanding of this second region is not trivial. The main focus of this work is to review the behavior of the low strain, nonlinear region and to report on new experiments meant to understand more about the behavior in the nonequilibrium regime.

\section{Invited Paper}

\section{$10: 20$}

3aPAa7. Scattering of Rayleigh wave by microcrack with interacting faces. Alexey M. Lomonosov (General Physics Institute, Vavilova str. 38, 119991 Moscow, Russian Federation, lom@kapella.gpi.ru), Peter Hess (Physical Chemistry Institute, University of Heidelberg, Im Neuenheimer Feld 253, 69120 Heidelberg, Germany, peter.hess@ urz.uni-heidelberg.de)

Scattering of short Rayleigh pulse by a partially closed surface-breaking microcrack is studied both experimentally and numerically. The microcrack was generated by a nonlinear surface wave with shock. In practice such type of cracks corresponds to fatigue or thermally generated ones. The crack was irradiated by a probe laser-generated Rayleigh. The scattered acoustic field at the surface, monitored by means of optical detector, consisted of the reflected and transmitted Rayleigh waves, longitudinal and shear acoustic waves excited by mode-conversions at the crack. Characteristic size of the crack was 50-100 $\mu \mathrm{m}$, experimental bandwidth was 5-200 MHz. Scattering was simulated numerically in finite differences. For modeling the partially closed crack we proposed a simple one-parameter model, which takes into account weak interaction between the crack faces. It was shown, that interaction changes the predicted frequency-dependent transmission and reflection coefficients significantly. On the basis of fitting the simulated acoustic field to the measured one, the evaluation of the crack size has been performed. In this work frequency dependence of the following three parameters were employed for that purpose: transmission and reflection coefficients, and the time delay of the transmitted pulse. Obtained estimates were compared to the direct microscopic measurement of the crack.

\section{Contributed Papers}

10:40

3aPAa8. Finite element simulation of the linear and nonlinear interaction of progressive acoustic waves with a standing acoustic wave field. Ebrahim Lamkanfi (Ghent University, Sint-Pietersnieuwstraat 41, 9000 Ghent, Belgium, Ebrahim.Lamkanfi@ugent.be), Nico F. Declercq (Georgia Tech Lorraine - G.W. Woodruff School of ME, UMI Georgia Tech - CNRS 2958, 2 rue Marconi, 57070 Metz, France, nico.declercq@me.gatech.edu), Wim Van Paepegem (Ghent University, Sint-Pietersnieuwstraat 41, 9000 Ghent, Belgium, wim .vanpaepegem@ugent.be), Joris Degrieck (Ghent University, SintPietersnieuwstraat 41,9000 Ghent, Belgium, joris.degrieck@ugent.be)

Throughout the 20th century studies have been published on the scattering of sound by sound. It has been shown that a combination of nonlinear effects and diffraction effects exists. Approximate models have been developed and some have been verified by experiments. The report presents a finite element simulation of the effect and results in exact solutions for configuration under consideration. It is shown that, depending on the amplitude of the standing wave field, orthogonally incident sound waves diffract. A simplified model is developed for linear interactions, followed by a more realistic model incorporating nonlinear elastic effects. The study is performed in a liquid and also in a solid. Analysis is achieved by Fourier transformation and a comparison is made with diffraction laws governed by the grating equation. For simplicity a $2 \mathrm{D}$ model is presented that is extendible however to a $3 \mathrm{D}$ problem should that be necessary. Comparison with some of the earlier published approximate results is also performed. The study shows the detailed influence of the amplitude on the strength of the diffraction effect.

\section{1:00}

3aPAa9. Use of synchronous nonlinear interaction of flexural Lamb waves in NDT-application. Maria Y. Izosimova (Center for Industrial and Medical Ultrasound, Applied Physics Lab., University of Washington, 1013 NE 40th St., Seattle, WA 98105, USA, maria.izossimova@mail .ru), Alexandr I. Korobov (Dept. of Acoustics, Physics Faculty, M.V. Lomonosov Moscow State University, Leninskie gory 1, 119991 Moscow, Russian Federation, akor@acs465a-1.phys.msu.ru), Dmitrii Mekhedov (Center for Industrial and Medical Ultrasound, Applied Physics Lab., University of Washington, 1013 NE 40th St., Seattle, WA 98105, USA, akor@acs465a.phys.msu.ru)

Workability of synchronous nonlinear interaction of flexural Lamb waves in NDT-application is demonstrated. During experiments traveling and standing $\mathrm{A}_{0}$ waves were used. Lamb waves are dispersive, so to provide synchronous nonlinear mode for traveling waves, non-collinear interaction of two waves with frequency $f$ were realized in aluminum alloy plate with defects. In the area of waves' nonlinear interaction, traveling wave of $2 f$ frequency appeared. Standing Lamb waves were excited with amplitudemodulated signal in a cylindrical resonator of aluminum plate. Carrier 
frequency $f$ and modulating frequency $F \ll f$ are equal to natural modes of the resonator. Spectrum of amplitude-modulated waves contained oscillations with frequencies $f, f+F$, and $f-F$. As a result of interaction, oscillation on heterodyne frequency $F$ appeared in the plate and excited a natural mode. Amplitudes of traveling and standing waves on heterodyne frequencies depend on presence of defects in investigated area (non-classic nonlinearity).
Spatial distribution of amplitudes of initial waves and ones on heterodyne frequency in samples were measured with laser vibrometer PolytecPSV300. In area containing heterogeneities, amplitude increase of waves on heterodyne frequencies was observed. The possibility to define the spatial distribution of defects using measurements of heterodyne wave amplitude distribution was shown experimentally. Work supported by RFBR.

\section{Invited Papers}

\section{1:20}

3aPAa10. Microdamage detection and monitoring using nonlinear elastic wave spectroscopy over a wide frequency range. Bart Van Damme (K.U.Leuven Campus Kortrijk, E. Sabbelaan 53, 8500 Kortrijk, Belgium, Bart.vandamme@kuleuven-kortrijk .be), Pierre-Yves Le Bas (EES-11 (Geophysics) - Los Alamos National Laboratory, MS D443, Los Alamos, NM 87545, USA, pylb@lanl.gov), Koen Van Den Abeele (K.U.Leuven Campus Kortrijk, E. Sabbelaan 53, 8500 Kortrijk, Belgium, koen.vandenabeele @kuleuven-kortrijk.be)

Innovative diagnostic techniques based on Nonlinear Elastic Wave Spectroscopy (NEWS) have been implemented for global inspection of microdamage in intact and damaged aeronautical components using low and high frequency sound waves. For low frequency analysis, we focused on the amplitude dependent analysis of the resonance behavior, both in time and frequency domain. Alternatively, we have analyzed the time-windowed interaction of a hammer impact with a high frequency continuous wave. The results of both techniques agree extremely well, and prove their potential for quick and global microdamage detection. For local inspection, we have implemented a combination of the traditional elastic wave time reversal technique with a phase coded pulse sequence selective excitation. Alternatively, sparse array tomographic reconstruction with NEWS pre-treatment could be used. The nonlinearity based imaging techniques are illustrated on aeronautical components, and discussed in terms of the feasibility and usefulness of the methodologies as new tools for microdamage imaging.

\section{1:40}

3aPAa11. Simulations of nonlinear ultrasonic NDT of plate-like structures. Marieke Geeraert (K.U.Leuven Campus Kortrijk, E. Sabbelaan 53, 8500 Kortrijk, Belgium, Marieke.Geeraert@student.kuleuven.be), Els Janssen (K.U.Leuven Campus Kortrijk, E. Sabbelaan 53, 8500 Kortrijk, Belgium, Els.Janssen@kuleuven-kortrijk.be), Steven Delrue (K.U.Leuven Campus Kortrijk, E. Sabbelaan 53, 8500 Kortrijk, Belgium, steven.delrue@kuleuven-kortrijk.be), Olivier Bou Matar (IEMN, UMR CNRS 8520, avenue Poincaré, BP 60069, 59652 Villeneuve d'Ascq, France, olivier.boumatar@iemn.univ-lille1.fr), Erik Blomme (Katholieke Hogeschool Zuid-WestVlaanderen dept. VHTI, Doorniksesteenweg 145, 8500 Kortrijk, Belgium, Erik.Blomme@katho.be), Herbert De Gersem (K.U.Leuven Campus Kortrijk, E. Sabbelaan 53, 8500 Kortrijk, Belgium, Herbert.DeGersem@kuleuven-kortrijk.be), Koen Van Den Abeele (K.U .Leuven Campus Kortrijk, E. Sabbelaan 53, 8500 Kortrijk, Belgium, koen.vandenabeele@kuleuven-kortrijk.be)

Inclusions in materials generally affect the linear wave propagation characteristics of sound waves, observed through scattering, and reduced reflection or transmission with respect to pure materials. The smaller the inclusions, for a fixed ultrasonic frequency, the harder it is to interpret these observations. Therefore, zones of microdamage initiated from fatigue or thermal loading might not induce any linear scattering at all. Nevertheless, the damage information is definitely carried along within the propagating sound wave. In this case, however, it is necessary to investigate the spectral changes of the propagating sound waves, in the form of harmonics and intermodulation frequencies. This contribution reports on simulations of the nonlinear signatures arising in Lamb, Rayleigh and bulk wave propagation in media with localised microdamaged zones. The simulations are performed with a Finite Difference Time Domain solver accounting for microdamage though the inclusion of hysteretic nonlinear stress-strain relations at the microscale. Depending on the configuration it is possible to obtain one or more harmonic images of the defect, their number and relative position containing information about the location and depth of the defect. The simulations allow simple prediction of the response images in complicated experimental conditions.

\section{Contributed Paper}

\section{2:00}

3aPAa12. Fast and Slow Dynamics in Damaged Materials: Correlation to Acoustic Emission. Mourad Bentahar (ENSIM - LAUM, Université du Maine, rue Aristote, 72085 Le Mans, France, Mourad.Bentahar @univ-lemans.fr), Anne Marec (ENSIM - LAUM, Université du Maine, rue Aristote, 72085 Le Mans, France, anne.marec.etu@univ-lemans .fr), Rachid El Guerjouma (ENSIM - LAUM, Université du Maine, rue Aristote, 72085 Le Mans, France, Rachid.El_Guerjouma@univ-lemans .fr), Jean-Hugh Thomas (ENSIM - LAUM, Université du Maine, rue Aristote, 72085 Le Mans, France, jean-hugh.thomas@univ-lemans.fr)

Results are reported of the study of anomalous nonlinear fast dynamics (FD) and slow dynamics (SD) in damaged solids. Experimental observations characterizing FD and SD may be used to detect and eventually characterize induced damage of structural components. Here, we analyze the evolution of the non-linear dynamic behaviour of two polymer-based composites of different reinforcing elements as Sheet Moulding Compound (SMC) and Polymer Concrete (PC) as a function of their gradual damage. More particularly, non-linear FD and SD parameters (time and frequency shift) have been found to be very sensitive to damage creation and evolution for both materials. Besides,acoustic emission monitoring was performed and allowed to calculate the elastic energy released by SMC and PC at every damage stage. Interesting logarithm-like evolution of the followed non-linear parameters as a function of the calculated energy is found. A classification of the collected acoustic emission signals is proposed to understand the contribution of the different damage mechanisms to the evolution of the non-linear behaviour. 


\section{Contributed Papers}

$1: 40$

3aPAa13. Chirp-coded excitation applied with advanced pulse inversion for nonlinear acoustics in complex steel samples. Serge Dos Santos (ENI Val de Loire, Université François Rabelais de Tours, LUSSI CNRS, rue de la Chocolaterie, 41034 Blois, France, serge.dossantos @ univ-tours.fr), Thomas Goursolle (ENI Val de Loire, Université François Rabelais de Tours, LUSSI CNRS, rue de la Chocolaterie, 41034 Blois, France, thomas.goursolle@univ-tours.fr), Martin Drab (Czech Technical University in Prague, Trojanova 13, 12000 Praha 2, Czech Republic, drab@kepler.fjif.cvut.cz), Tomas Slunecko (ZD Rpety - DAKEL, Ohrobecka 408, 14200 Praha 4, Czech Republic, drab@kepler.fjif.cvut.cz)

Nonlinear acoustics for nondestructive testing is still an emergent technique in complex materials with localized cracks because linear effects can be cancelled using specific symmetries for the pre- and post-signal processing. The local nonlinear signature is accessible even in complex aeronautic structure by using the symmetry properties: i. e. time reversal invariance and pulse inversion associate to chirp-coded excitation. Excitation Symmetry Analysis Method [Dos Santos et al., JNLM, 2007] which is an extension of the pulse inverted method for extraction of third order nonlinearity is applied experimentally for the measurement of relative level of nonlinearity on a airplane steel bracket, on which visible crack was created by fatigue tests. This pre-processing method has been coupled to a 100-250 $\mathrm{kHz}$ bandwidth chirp-coded ultrasonic excitation, realized at various amplitude $\left(A, A / 2,3^{1 / 2} A / 2\right.$, etc.), and performed with DAKEL IDK09 $200 \mathrm{kHz}$ PZT sensors. Signal processing based on correlation measurements, allow an extraction of the nonlinear signature versus fatigue level. Experiments and data processing have been conducted with a 12 bit DAKEL-DTR system in the bandwidth $80-800 \mathrm{kHz}$ and compared with a continuous 4 channel acoustic emission device.

\section{2:00}

3aPAa14. Determination of symmetry relations between higher order material constants for the study of nonlinear acoustic properties of piezoelectric crystals of any symmetry class. Nico F. Declercq (Georgia Tech Lorraine - G.W. Woodruff School of ME, UMI Georgia Tech - CNRS 2958, 2 rue Marconi, 57070 Metz, France, nico.declercq@me.gatech.edu)

Each crystal is invariant under a given point symmetry operation. This results in relations between the different material property constants, such as second order elastic constants, second order piezoelectric constants and second order dielectric constants. Those relations are well reported in text books for the linear behavior of crystals. When nonlinear effects are studied, higher order constants play a significant role and their symmetry relations have only been reported in the literature for a limited number of crystal classes. The problem with higher order constants is that it is rather complicated and very time consuming to study the symmetry relations. This paper presents a newly developed semi-automatic computer program that is able to handle any kind of symmetry relation. Results are reported for all the different crystal classes for the higher order constants, i.e. third order elastic constants, third order piezoelectric constants, third order dielectric constants and electrostrictive constants. The results are important for the study of nonlinear properties of crystals and composites and will ease the experimental quantitative determination of all linear and nonlinear material constants of crystals.

\section{2:20}

3aPAa15. Nonlinearity, hysteresis, end-point memory, and congruence in sandstones. Kevin E. Claytor (Los Alamos National Laboratory, POBox 1663, Los Alamos, NM 87545, USA, ke.claytor@gmail .com), Joseph R. Koby (Los Alamos National Laboratory, POBox 1663, Los Alamos, NM 87545, USA, jkoby@lanl.gov), Jim A. Tencate (EES-11 (Geophysics) - Los Alamos National Laboratory, MS D443, Los Alamos, NM 87545, USA, tencate@lanl.gov)

Very low frequency stress-strain measurements on many rocks show definite and repeatable hysteresis loops. Much of the modeling of this behavior uses Preisach or Ising-like models adapted from models developed for magnetic systems which exhibit hysteresis. To date, the experimental data on many rocks showing end-point memory are noisy and only qualitative. The congruence property, in fact, has never been examined for rocks. Just how well does the behavior of a sandstone fit a Preisach model? To find the answer to this question, we performed a new set of careful stressstrain experiments on several sandstones to examine end-point memory (in detail) and also to see if the congruence property does indeed hold for these rocks. In addition we explored the dependence of measurement rate on the relaxation time of these rocks (the analog to magnetic aftereffect). For very slow measurement rates (low frequencies), hysteresis disappears, leaving only a simple nonlinear stress-strain curve.

\section{2:40}

3aPAa16. Foundations of the ultrasonic non-destructive method of determination of stresses in near-the-surface layers of solids. Alexander Guz (Institute of Mechanics, Nesterov str. 3, Kiev, 03057 Kiev, Ukraine, guz@ carrier.kiev.ua)

The ultrasonic non-destructive method under consideration is intended for measurements of uniaxial and two axial stresses in the near-the-surface layers of relatively rigid materials (metals, alloys and similar material). This method is intended for measurements of the actual, assemble, operating, preload prestress and other stresses. Above mentioned stresses must be considered as initial or residual stresses taking into account the theory [1] under consideration. In this case the disturbances (displacements and stresses of the 3-D linearized theory [1] of elastic waves in bodies with initial or residual stresses) arise due to ultrasonic vibrations. Description of the nondestructive method under consideration and information on instruments and devices for measurements are presented. Some examples of non-destructive determination of stresses in near-the-surface layers of materials are presented also as applied to the residual stresses arising at electric welding and to the operating stresses arising at loading. Additional information is presented in monograph [1]. References: 1.Guz A.N. Elastic waves in bodies with initial (residual) stresses. Kiev: "A.C.K." Publishers, 2004. - 672 p. (In Russian).

\section{3:00}

3aPAa17. Effect of Water Saturation on the Nonlinear Elastic Response of Concrete. Cédric Payan (LCND - Université de la Méditerranée, IUT Aix Provence, Avenue Gaston Berger, 13625 Aix en Provence Cedex, France, cedric.payan@univmed.fr), Vincent Garnier (LCND - Université de la Méditerranée, IUT Aix Provence, Avenue Gaston Berger, 13625 Aix en Provence Cedex, France, vincent.garnier@univmed.fr), Joseph Moysan (LCND - Université de la Méditerranée, IUT Aix Provence, Avenue Gaston Berger, 13625 Aix en Provence Cedex, France, joseph.moysan @univmed.fr)

Nonlinear interaction of a monochromatic elastic wave with a low frequency should be a good tool for non-destructive evaluation of existing concrete structures. This technique has already proofed efficient in detecting global damage. However, it is necessary to understand the influence of some structural parameters as porosity, stress state, or water saturation on the nonlinear processes. In this way, a thermodynamically based model containing all of them is presented in this paper. It is sustained by nonlinear interaction measurements in order to quantify the evolution of the nonlinear behaviour of concrete with water saturation state.

\section{3:20}

3aPAa18. The Parametric Array in Berea Sandstone, Revisted. Pierre-Yves Le Bas (EES-11 (Geophysics) - Los Alamos National Laboratory, MS D443, Los Alamos, NM 87545, USA, pylb@lanl.gov), Jim A. Tencate (EES-11 (Geophysics) - Los Alamos National Laboratory, MS D443, Los Alamos, NM 87545, USA, tencate@lanl.gov)

Previous measurements of the characteristics of the parametric array in sandstone by Johnson and Shankland [J. Geophys. Res., 94, 17729-17734 (1989)] were difficult to perform and, given the apparatus of the day, only qualitative. Since then, memory effects have been found to play a strong role in the dynamic behavior of rocks. This experimental study was performed to 
find out how well the "classical" theory of nonlinear acoustics, i.e., all compressional waves with no memory effects, works at describing the parameteric array in a granular solid. An array of PZT disk transducers (every other one broadcasting one of two frequencies) was epoxied to a large block of Berea sandstone $(1.2 \times 0.4 \times 0.4 \mathrm{~m})$. The two primary frequencies were near $100 \mathrm{kHz}$ and chosen to produce a difference frequency around $15 \mathrm{kHz}$. Using a scanning laser vibrometer (Polytec), several scans of the beampatterns were made at the opposite end of the rock and the results compared with calculations based on those commonly used for parametric arrays in water. A narrow beam generated from the two primaries was measured and the beampattern compared with those of the primaries. The agreement is surprisingly good using a beta of around 200 and a Q of about 50.

WEDNESDAY MORNING, 2 JULY 2008

ROOM 351, 8:00 TO 11:00 A.M.

\title{
Session 3aPAb
}

\section{Physical Acoustics: Acoustic Probes of Planetary Environments I}

\author{
Andi Petculescu, Cochair \\ University of Louisiana, Department of Physics, PO Box 44210, Lafayette, LA 70504, USA \\ Martin C. Towner, Cochair \\ PSSRI, The Open University, Walton Hall, Milton Keynes, MK7 6AA, UK
}

Invited Paper

8:00

\begin{abstract}
3aPAb1. The atmospheres of Mars, Venus and Titan: observed and modelled structures. Sebastien Lebonnois (Laboratoire de Meteorologie Dynamique, IPSL/CNRS, 4 place Jussieu, box 99, 75252 Paris Cedex 05, France, sllmd@1md.jussieu.fr)

In our solar system, three bodies possess atmospheres presenting similar characteristics with our own environment: our neighbours Mars and Venus, and also Saturn's largest satellite Titan. The comparative study of these different atmospheric systems provides many insights on their respective behaviour, and helps understand the processes at work within these complex systems. In this review, the observed vertical and latitudinal structures of these atmospheres are presented, including their variability and seasonal evolution. Waves that have been observed are also shown, e.g., thermal tides, gravity waves. Global Climate Models that have been developed to study such atmospheric systems are also presented. Using these tools, production and role of the different waves present in each atmosphere may be studied.
\end{abstract}

\section{Contributed Paper}

\section{8:20}

3aPAb2. Bach on other planets or why acoustic sensors make sense in planetary science. Andi Petculescu (University of Louisiana, Department of Physics, PO Box 44210, Lafayette, LA 70504, USA, andi @ louisiana.edu), Timothy G. Leighton (Institute of Sound and Vibration, Univ. of Southampton, University Road, Highfield, SO17 1BJ Southampton, UK, T.G.Leighton@soton.ac.uk)

The European Huygens probe, which landed on Titan in 2005, is the only planetary probe so far to incorporate passive and active acoustic sensing. Acoustic wave motion is intimately coupled with an atmosphere and thus can give first-hand information on that environment. Motivation for acoustic sensing in planetary atmospheres abounds: anemometry, turbulence measurements, soil characterization, sound generation by lightning, acoustics of liquid hydrocarbon flows, cryovolcanoes, dust devils, quantitative fluid spectroscopy, thermometry, atmospheric boundary layers etc. In this paper it is shown quantitatively how the environments of Mars, Venus, and Titan affect the generation and propagation of audible sound differently. To exemplify, a musical fragment with a rich frequency content was chosen (Bach's Toccata and Fugue in D-minor played by one of the authors) and passed through the three planetary "filters." Appreciation of the nature of these sounds will inform the design of acoustic sensors for future space probes. Furthermore, a demonstration that the physics is sufficiently understood to predict these sounds indicates that the same understanding can be applied quantitatively to interpret alien sounds to estimate the environmental conditions.

\section{Invited Papers}

\section{8:40}

3aPAb3. Use of microphones to record windspeed and turbulence in planetary environments. Ralph D. Lorenz (JHU Applied Physics Lab, 11100 Johns Hopkins Road, Laurel, MD 20723, USA, ralph.lorenz@jhuapl.edu)

A major feature of any planetary acoustic measurement is noise due to ambient winds, or to the motion of the measurement platform through the air. Venera 13 and 14 recorded sound on the surface of Venus: the amplitude of the microphone signal was interpreted (by dynamic pressure scaling) to recover a windspeed. The Huygens probe at Titan also recorded sound levels during descent and on the surface with a passive microphone and an acoustic sounder. The author has made passive microphone measurements made on a rotating aerospace vehicle (an instrumented Frisbee) which show spin modulation and a variation with flight speed and angle of attack. He has also used microphones as a crude wind sensor on an array of 20 meteorological stations used to study dust devils in Arizona. All of these observations will be reviewed. Lessons learned for future planetary measurements, and prospects and methods for microphonic anemometry (especially with limited telemetry bandwidth) will be discussed. 
3aPAb4. The Huygens Surface Science Package sound speed measurements and the methane content of Titan's atmosphere. A. Hagermann (PSSRI, Open University, Walton Hall, MK7 6AA Milton Keynes, UK, unknown@unknown.com), Martin C. Towner (PSSRI, The Open University, Walton Hall, MK7 6AA Milton Keynes, UK, m.c.towner@open.ac.uk), Philip D. Rosenberg (PSSRI, Open University, Walton Hall, MK7 6AA Milton Keynes, UK, p.d.rosenberg@open.ac.uk), James R. Garry (PSSRI, Open University, Walton Hall, MK7 6AA Milton Keynes, UK, jrcgarry@hotmail.com), Hakan Svedhem (European Space Agency, Keplerlaan 1, NL 2201 AZ Noordwijk ZH, Netherlands, hsvedhem@rssd.esa.int), Mark R. Leese (PSSRI, Open University, Walton Hall, MK7 6AA Milton Keynes, UK, m.r.leese@open.ac.uk), Brijen Hathi (PSSRI, Open University, Walton Hall, MK7 6AA Milton Keynes, UK, b.hathi@open.ac.uk), Ralph D. Lorenz (JHU Applied Physics Lab, 11100 Johns Hopkins Road, Laurel, MD 20723, USA, ralph.lorenz@jhuapl.edu), John C. Zarnecki (PSSRI, Open University, Walton Hall, MK7 6AA Milton Keynes, UK, j.c.zarnecki @ open.ac.uk)

The Huygens probe descended through Titan's atmosphere in January 2005. On board was the Surface Science Package (SSP), a set of nine sensors, which included a speed-of-sound sensor. We present a detailed description of the SSP speed of sound measurements and report constraints on the methane content in Titan's lower atmosphere based on these measurements. After extensive instrument calibration and subsequent Bayesian analysis of the data, the most likely result derived from our measurements in Titan's lower atmosphere is a methane fraction of approximately $2 \%$ at $10 \mathrm{~km}$, increasing to $3.5 \%$ at lower altitudes. These estimates are based on a binary composition. Our data show that any large scale variation of methane within the lower $11 \mathrm{~km}$ of Titan's atmosphere is unlikely. Within experimental and theoretical uncertainties, our estimates are lower than, but compatible with earlier estimates obtained from the mass spectrometry experiment.

\section{9:20}

3aPAb5. Waterfalls in space, and other problems of 'underwater' acoustics on a small planet. Timothy G. Leighton (Institute of Sound and Vibration, Univ. of Southampton, University Road, Highfield, SO17 1BJ Southampton, UK, T.G.Leighton @ soton.ac.uk), Paul R. White (Institute of Sound and Vibration, Univ. of Southampton, University Road, Highfield, SO17 1BJ Southampton, UK, prw@isvr.soton.ac.uk), Daniel C. Finfer (Institute of Sound and Vibration, Univ. of Southampton, University Road, Highfield, SO17 1BJ Southampton, UK, d.c.finfer@soton.ac.uk)

Whilst extraterrestrial liquids do occur in the Solar System, today's acoustical oceanographers have fewer sites to which they can apply their experience of Earth's oceans than perhaps they would have had in the early Solar System, with its magma oceans. Possible sites are Saturn's moons Titan and Enceladus, and Jupiter's moon Europa. The ability to transfer our understanding of Earth's acoustical oceanography to other moons and planets is particularly valuable, given that current understanding is sufficient to undertake complex inversions to estimate Earth's ocean environmental parameters from relatively sparse, or even naturally-occurring, acoustical signals. However such transference should be done with care, as terms familiar in Earth's acoustical oceanography may not be correct on other worlds. For example, in a deep ocean on a small world (such as Europa), the hydrostatic pressure will not equal the product of the density, the depth, and the surface value of the acceleration due to gravity, since the latter can vary with depth, and because vertical lines are not parallel on a small planet. This paper explores two cases of transferring our terrestrial experience off world, to the ice seas of Europa, and to the methane lakes and waterfalls of Titan.

\section{9:40-10:00 Break}

\section{0:00}

3aPAb6. Acoustic Anemometry on Mars. Jean-Pierre Williams (California Institute of Technology, Division of Geological and Planetary Sciences, MC 150-21, Padadena, CA 91125, USA, jpierre@gps.caltech.edu), Ian McEwan (California Institute of Technology, Division of Geological and Planetary Sciences, MC 150-21, Padadena, CA 91125, USA, ijm@gps.caltech.edu)

Acoustic anemometers provide wind measurements with high sensitivity, temporal resolution, and accuracy, and are in many ways superior to the hot wire technique anemometers currently and previously flown to Mars. An anemometer is under development that employs an internal model of how sound is changed by a particular gas mixture with respect to wind speed and temperature to derive these values. This is done by measuring the transfer function (attenuation and phase versus frequency) of sound between two transducers with a very wide spread-spectrum signal and comparing this with its internal model. While sonic anemometry is the premier technique for research studies of winds on Earth, the acoustic properties differ substantially from atmosphere to atmosphere and it is not valid to assume that an existing terrestrial system can provide heritage for a planetary instrument. Further, current multi-gas acoustic models rely on constants derived from experiments performed in conditions far from the planetary application intended here. Since the parameters of the model must be well defined in our approach, the instrument's ability to accurately measure transfer functions will be used in experiments to improve our knowledge of the behavior of various calibrated gas mixtures over relevant temperatures and pressures.

\section{0:20}

3aPAb7. An ultrasonic anemometer for Mars. Colin Wilson (University of Oxford, Department of Atmospheric, Oceanic and Planetary Physics, OX1 3PU Oxford, UK, wilson@atmos.ox.ac.uk), Lee A. Davis (University of Warwick, School of Engineering, CV4 7AL Coventry, UK, lee.davis@warwick.ac.uk), David Hutchins (University of Warwick, School of Engineering, CV4 7AL Coventry, UK, D.A.Hutchins@warwick.ac.uk), Martin C. Towner (PSSRI, The Open University, Walton Hall, MK7 6AA Milton Keynes, UK, m.c.towner@open.ac.uk)

Ultrasonic anemometers are often used for studies of 3-D atmospheric turbulence on Earth, due to their robust calibration and fast operation $(>10 \mathrm{~Hz})$. The same qualities make ultrasonic anemometry attractive for use on Mars, where similar atmospheric turbulence is found. The low density of Martian atmosphere - a hundredth that of the Earth's - is problematic, because of the large acoustic impedance mismatch between the atmosphere and piezoelectric transducers. One solution to this problem is to use piezoelectric transduc- 
ers with a hornlike resonator bonded to their front surface. Another solution is to use a capacitive membrane transducer instead. We report on performance of various ultrasonic transducers in carbon dioxide Martian pressures, and present an instrument design capable of meeting the stringent mass, power, and environmental requirements of the European ExoMars lander.

10:40

3aPAb8. Solid-fluid coupling on planets: from seismology to acoustics and beyond. Raphael F. Garcia (DTP-OMP Univ. Toulouse, 14 Avenue E. Belin, 31400 Toulouse, France, garcia@dtp.obs-mip.fr), Philippe Lognonné (IPGP, 4 avenue de Neptune, 94107 Saint Maur des Fossés, France, lognonne@ipgp.jussieu.fr), Giovanni Occhipinti (IPGP, 4 avenue de Neptune, 94107 Saint Maur des Fossés, France, ninto@ipgp.jussieu.fr)

On Earth, solid-fluid coupling is responsible for acoustic signals created by quakes or volcanic eruptions which have been observed by infrasonic sensors or through ionospheric perturbations produced by infrasounds. Similarly volcanic explosions, ocean surface waves and ocean internal gravity waves are producing seismic signals. A specific normal mode coupling theory has been developed for the whole solid/ocean/atmosphere Earth system in order to model these phenomena. Recent developments for the observation and modeling of the infrasonic waves created by quakes and tsunamis have focused on the ionospheric perturbations produced by the exponential amplification of vertically propagating infrasonic and gravity waves. On Earth, these tools are particularly interesting for the observation of seismic surface waves and tsunamis in the open ocean where sensors are not present. However, the interest is even stronger to infer the internal structure of planets for which the deployment of seismometers is almost impossible. Theoretical modeling of solid/atmosphere coupling on Venus is presented, including open questions concerning the attenuation of infrasounds by Venus atmosphere. An attempt to observe quake signals with VIRTIS instrument on board Venus Express ESA mission is also described. Finally, a mission analysis tool and other ideas for future planetary investigations of atmospheric acoustic normal modes are discussed.

WEDNESDAY MORNING, 2 JULY 2008

ROOM 352A, 8:00 A.M. TO 1:00 P.M.

\title{
Session 3aPAc
}

\section{Physical Acoustics and Computational Acoustics: Combustion Noise and Thermo-Acoustics I}

\author{
Tim Lieuwen, Cochair \\ School of Aerospace Engineering, Georgia Institute of Technology, Atlanta, Georgia 30332-0150, USA \\ Maria A. Heckl, Cochair \\ Keele University, Staffordshire, Newcastle-under-Lyme, ST5 5BG, UK \\ Rafael Piscoya, Cochair \\ Technische Fachhochschule Berlin, Univ. of Applied Sciences, Luxemburger Str. 10, Berlin, 13353, Germany
}

Invited Papers

\begin{abstract}
8:00
3aPAc1. Sound production by partially premixed flames in a stagnation flow. Thierry Schuller (Ecole Centrale Paris, Laboratoire EM2C - CNRS, Grande-Voie des Vignes, 92295 Chatenay-Malabry, France, thierry.schuller@em2c.ecp.fr), Patrick Duchaine (Ecole Centrale Paris, Laboratoire EM2C - CNRS, Grande-Voie des Vignes, 92295 Chatenay-Malabry, France, patrick.duchaine @em2c.ecp.fr), Laurent Zimmer (Ecole Centrale Paris, Laboratoire EM2C - CNRS, Grande-Voie des Vignes, 92295 ChatenayMalabry, France, laurent.zimmer@em2c.ecp.fr)

In the perspective of combustion noise reduction, understanding mechanisms of sound generation from combustion of non perfectly mixed reactants is a major challenge. Sound production is thus investigated for vortices impinging a flat laminar flame in a stagnation flow. Such a configuration helps to better understand sound production from turbulent flames. A study with different compositions of the main flow and surrounding gases - mixtures of nitrogen, air and methane - is carried out under the same mean flow and perturbations conditions. Time traces of radiated sound pressure and chemiluminescence emission from radical species present in the reaction zone are characterized together with detailed imaging of the flame and flow motions. Elements of classical combustion noise theory are used to identify the main noise sources when air or fuel is entrained by the vortex interacting with the flame.
\end{abstract}


3aPAc2. Flow field and acoustic characteristics of an acoustically forced burner impinging jet. Yang Zhang (The University of Manchester, School of Mechanical, Aerospace and Civil Engineering, George Begg Building, M60 1QD Manchester, UK, yang.zhang@manchester.ac.uk), Saad Hajim (The University of Manchester, School of Mechanical, Aerospace and Civil Engineering, George Begg Building, M60 1QD Manchester, UK, saad.hajim@manchester.ac.uk), Jason Bassi (The University of Manchester, School of Mechanical, Aerospace and Civil Engineering, George Begg Building, M60 1QD Manchester, UK, jason.bassi @manchester.ac.uk), S Farhat (The University of Manchester, School of Mechanical, Aerospace and Civil Engineering, George Begg Building, M60 1QD Manchester, UK, s.farhat@manchester.ac.uk)

The flow field and acoustic characteristics of a turbulent impinging jet from an impinging burner have been investigated experimentally. The effect of acoustically excited upstream flow with a range of driving frequencies from 0 to $3000 \mathrm{~Hz}$ and use of perforated plate near the nozzle have been studied to achieve modifications to the near-field and far-field of the jet. The acoustic response of the burner to upstream acoustically excited flow under different geometry configurations was investigated in detail. The burner components were found to have a significant effect on the burner acoustic response, with overall damping and enhancement achieved over the full driving frequency range. The velocity flow field of the impinging jet, including spectra and rms data, were mapped from the burner nozzle to the plate with CTA measurements with three acoustic excitation frequencies and no excitation; $0 \mathrm{~Hz}, 52.5 \mathrm{~Hz}, 75 \mathrm{~Hz}$ and $530 \mathrm{~Hz}$ (within acoustic mode bands of burner).The results show that excitation frequencies of $52.5 \mathrm{~Hz}$ and $75 \mathrm{~Hz}$ were found to propagate all the way to the plate, whilst $530 \mathrm{~Hz}$ dissipates near the nozzle exit. ( $\mathrm{Re}=16,200, \mathrm{H} / \mathrm{D}=3$, nozzle velocity profile).

\section{$8: 40$}

3aPAc3. Prediction of Self Excitation Frequencies and Amplitudes for a Model Gas Turbine Burner. Catherine Gardner (Queen Mary University of London, School of Engineering and Material Science, Mile End Road, E1 4NS London, UK, catherine .gardner@qmul.ac.uk), S. M. Reza Hosseini (Queen Mary University of London, School of Engineering and Material Science, Mile End Road, E1 4NS London, UK, s.m.hosseini@qmul.ac.uk), Chris J. Lawn (Queen Mary University of London, School of Engineering and Material Science, Mile End Road, E1 4NS London, UK, c.j.lawn@qmul.ac.uk)

Forced excitation of a swirl stabilised methane/air flame by acoustic waves at atmospheric pressure has been characterised in order to show that the frequencies and amplitudes where self-excitation occurs, but the natural limit cycle takes effect, can be predicted from these data. Chemiluminescent emission was therefore recorded to measure the Flame Transfer Function (FTF), as the flame was acoustically excited by two loud speakers. The experiments covered a wide range of frequencies and amplitudes with particular emphasis on the FTF at high amplitudes of excitation, where a non-linear response is often reported. The system was modelled by the 1D thermoacoustic element method, in which each acoustic element, such as a duct or a contraction, is described by a four-pole matrix. The flame is incorporated as another four-pole matrix that describes the measured flame transfer function. The solution of this set of equations without forcing predicts self-excitation frequencies and amplitudes that can be compared with the experimental data. The minimum magnitudes of the FTF for excitation may also be calculated. When proven at this small scale, the procedure will be applied to predict the limit cycling of actual installations from rig tests.

3aPAc4. Low-order modelling of unsteady flame behaviour in a spray combustor. David M. Arthur (University of Cambridge, Department of Engineering, Trumpington Street, CB2 1PZ Cambridge, UK, david.arthur@ cantab.net), Ann P. Dowling (University of Cambridge, Department of Engineering, Trumpington Street, CB2 1PZ Cambridge, UK, apd1@ cam.ac.uk), Stewart Cant (University of Cambridge, Department of Engineering, Trumpington Street, CB2 1PZ Cambridge, UK, rsc10@eng.cam.ac.uk)

The effect of mass flow rate perturbations on the stability of a liquid-fulled annular aeroengine gas turbine is investigated using a three-dimensional uRANS code. The simulations are performed on a single sector of an idealised annular gas turbine geometry. A Lagrangian fuel spray description is used with a Monte Carlo solution of Williams' spray equation. Broadband mass flow fluctuations are imposed at the combustor inlet and dilution ports, and the effect on heat release is quantifed using flame transfer functions. The excitation of inlet and dilution flows leads to signifcant unsteady combustion and the implications for self-excited oscillations are considered. The numerical results are then compared to a low-order model which is developed to account for the different physical mechanisms responsible for unsteady heat release when the inlet and dilution port air mass flow rates are perturbed. The low-order model is used to predict the possibility of self-excited oscillations as well as to investigate the potential of altering the phase and relative magnitude of the inlet and dilution port excitation as a possible means of controlling thermoacoustic oscillations.

\section{9:20}

3aPAc5. Mode switching of combustion instabilities associated with flame transfer function non-linearities. Daniel Durox (Ecole Centrale Paris, Laboratoire EM2C - CNRS, Grande-Voie des Vignes, 92295 Chatenay-Malabry, France, daniel.durox@em2c.ecp.fr), Nicolas Noiray (Ecole Centrale Paris, Laboratoire EM2C - CNRS, Grande-Voie des Vignes, 92295 Chatenay-Malabry, France, nicolas.noiray@em2c.ecp.fr), Thierry Schuller (Ecole Centrale Paris, Laboratoire EM2C - CNRS, GrandeVoie des Vignes, 92295 Chatenay-Malabry, France, thierry.schuller@em2c.ecp.fr), Sebastien Candel (Ecole Centrale Paris, Laboratoire EM2C - CNRS, Grande-Voie des Vignes, 92295 Chatenay-Malabry, France, sebastien.candel@em2c.ecp.fr)

Studies of combustion instabilities indicate that the frequency of oscillation changes during the growth of oscillations before a limit cycle is reached. It is also found that a system can switch from one mode to another when the amplitude exceeds a certain threshold. It is shown that these features can be linked to the flame response non-linearity. The analysis is carried out by examining combustion instabilities appearing on a multipoint injection burner, placed a the end of a cylindrical manifold. This element acts like a resonator and its length can be modified easily. Experiments indicate that mode switching takes place between two resonant modes of the cavity and this is shown to be caused by the non-linearity of the flame response. The phenomenon can be analyzed by considering the non-linear 
evolution of the flame transfer function. It is possible to combine this information with a stability analysis of the burner, and define conditions which give rise to mode switching. It is found that this behavior is related to a change in phase difference between heat release rate fluctuations and velocity perturbations at the burner outlet. This phase shift takes place as the amplitude of oscillation increases.

\section{9:40}

3aPAc6. Impact of acoustic boundary conditions on confined combustion noise. Ammar Lamraoui (Ecole Centrale Paris, Laboratoire EM2C - CNRS, Grande-Voie des Vignes, 92295 Chatenay-Malabry, France, ammar.lamraoui@em2c.ecp.fr), Franck Richecoeur (Ecole Centrale Paris, Laboratoire EM2C - CNRS, Grande-Voie des Vignes, 92295 Chatenay-Malabry, France, franck.richecoeur@em2c.ecp.fr), Thierry Schuller (Ecole Centrale Paris, Laboratoire EM2C - CNRS, Grande-Voie des Vignes, 92295 Chatenay-Malabry, France, thierry.schuller@em2c.ecp.fr), Sébastien Ducruix (Ecole Centrale Paris, Laboratoire EM2C - CNRS, Grande-Voie des Vignes, 92295 Chatenay-Malabry, France, sebastien.ducruix @ecp.fr)

The noise produced by aircraft engines at take-off and landing can be very harmful for airport neighbors, an issue that became recently particularly crucial. While combustion instabilities, where a strong coupling between flames and acoustic modes of the combustion chamber cause very high levels of harmonic sound, the wide bandwidth noise emitted by confined flames in stable situations is less well-documented. It has been recently shown by Tran et al. (2007) that varying the burner upstream acoustic boundary condition was a very efficient method for damping combustion instabilities and creating ideal conditions for the study of combustion noise. In the present study, the noise level induced by stable combustion is investigated for various upstream conditions. The test bench consists in a swirl stabilized burner, connected to a rectangular combustion chamber, equipped with microphone ports for acoustic measurements. The $40 \mathrm{~kW}$ burner is composed of two identical stages fed with air and propane. A photomultiplier equipped with a narrow band filter measuring the $\mathrm{CH}^{*}$ spontaneous emission from the flame is used to study heat release fluctuations in the flame. Time-resolved measurements are carried out and spectral analysis of pressure measurements and light emissions are performed for various inlet impedances.

\section{0:00-10:20 Break}

10:20

3aPAc7. Green's function model for a Rijke tube with a distributed heat source. Maria A. Heckl (Keele University, Staffordshire, ST5 5BG Newcastle-under-Lyme, UK, m.a.heckl@keele.ac.uk)

A Rijke tube is an open-ended tube with a compact heat source (flame or hot gauze) inside. The heat source is commonly modelled by the "n $\tau$ law", which assumes a point source releasing heat at a rate that is proportional to the velocity (delayed by a time lag $\tau$ ) at the heat source. The present paper aims to extend this model to include distributed heat sources. A distributed source is simulated by a row of point sources with individual heat release rates and time lags. The acoustic processes in the tube will be modelled by Lighthill's acoustic analogy equation, combined with a Green's function approach. The occurrence of thermo-acoustic instabilities will be predicted for different properties of the heat source. Geometrical complications in the tube, such as a blockage and a jump in cross-section, as well as a jump in mean temperature, will be taken into account.

\section{0:40}

3aPAc8. Experimental and Numerical Investigation of the Acoustic Response of Multi-slit Bunsen Burners. Philip De Goey (Eindhoven Univesity of Technology, Den Dolech 2, 5600 MB Eindhoven, Netherlands, 1.p.h.d.goey@tue.nl), Victor Kornilov (Eindhoven Univesity of Technology, Den Dolech 2, 5600 MB Eindhoven, Netherlands, v.n.kornilov@tue.nl), Ronald Rook (Eindhoven Univesity of Technology, Den Dolech 2, 5600 MB Eindhoven, Netherlands, r.rook@tue.nl), Jan Ten Thije Boonkkamp (Eindhoven Univesity of Technology, Den Dolech 2, 5600 MB Eindhoven, Netherlands, tenthije@win.tue.nl)

Acoustic resonances in combustion systems like central heating boilers prohibit further technological advances in these systems. The design and construction is obstructed by acoustic problems because they are largely misunderstood, in spite of our increase in knowledge over the last decades. The flame often acts as an active element in the acoustic field, because the flame transfer function of acoustic waves has a large amplitude at low frequencies. Current models of the phase of the flame transfer function of Bunsen-type flames, based on kinematic behavior of the flame dynamics, completely miss the experimentally observed phase, unless the measured flow field is used in the model. In the current paper we analyze numerical results of the flame dynamics, flow field and flame transfer function found with a 2D detailed numerical model of the flow and structure of the flame on a multiple-slit burner. The model is validated with experiments of the flame dynamics (using chemiluminescence), flow dynamics (using PIV) and flame transfer function (using $\mathrm{OH}$ luminescence for the heat release fluctuations and heated wire probe for the acoustic distortions) on exactly the same configuration. A very good agreement is found which indicates the importance of predicting all the influences of the flow on the flame and vise-versa.

\section{1:00}

3aPAc9. Numerical prediction of interaction between combustion, acoustics and vibration in gas turbines. Artur Pozarlik (University of Twente, P.O. Box 217, 7500 AE Enschede, Netherlands, a.k.pozarlik@utwente.nl), Jim B. Kok (University of Twente, P.O. Box 217, 7500 AE Enschede, Netherlands, j.b.w.kok@utwente.nl)

The turbulent flame in the lean combustion regime in a gas turbine combustor generates significant thermo-acoustic instabilities. The flame can amplify fluctuations in the released heat, and thus in the acoustic field as well. The induced pressure oscillations will drive vibrations of the combustor walls and burner parts. Stronger fluctuating pressure results in stronger fluctuations in the wall structure. Due to fatigue the remaining life time of the hard ware will be reduced significantly. This paper investigates modeling of acoustic oscillations and mechanical vibrations induced by lean premixed natural gas combustion. The mutual interaction of the combustion processes, induced oscillating pressure field in the combustion chamber, and induced vibration of the liner walls are investigated with 
numerical techniques. A partitioned procedure is used here: CFX-10 for the CFD analysis and Ansys-10 for the CSD analysis are coupled to give insight into a correlation between acoustic pressure oscillations and liner vibrations. These results will be compared with the available experimental data. The data are gathered in a purpose built $500 \mathrm{~kW} / 5$ bar premixed natural gas test rig.

11:20

3aPAc10. Influence of Temperature Gradients on the Sound radiated from Flames. Rafael Piscoya (Technische Fachhochschule Berlin, Univ. of Applied Sciences, Luxemburger Str. 10, 13353 Berlin, Germany, piscoya@tfh-berlin.de), Martin Ochmann (Technische Fachhochschule Berlin, Univ. of Applied Sciences, Luxemburger Str. 10, 13353 Berlin, Germany, ochmann@tfh-berlin.de)

The far field pressure of a turbulent flame can be determined using the standard boundary element method (BEM) if the sound pressure or its derivative is known at a closed surface (control surface) surrounding the flame, as long as the medium outside the control surface is homogeneous. If temperature gradients are present, the homogeneous Helmholtz equation is no more valid. In that case, the wave equation can be rewritten in form of an inhomogeneous Helmholtz equation with a source term that depends also on the unknown pressure. Using the "Dual Reciprocity BEM" the integral form of this wave equation can be solved involving only surface integrals, so that the sound field can still be computed from field values at the control surface. The cases under study consider a volume of hot gas with a temperature distribution that is prescribed or obtained from a CFD simulation. The influence of the temperature gradients on the sound field can be evaluated by comparison of characteristic quantities like sound power and radiation patterns, with and without temperature gradient.

\section{1:40}

3aPAc11. Localization of Sound Sources in Combustion Chambers. Christian Pfeifer (Institut für Strömungsmechanik und Technische Akustik, Technische Universität Berlin, Mueller-Breslau-Str. 8, 10623 Berlin, Germany, christian.pfeifer@pi.tu-berlin.de), Jonas P. Moeck (Institut für Strömungsmechanik und Technische Akustik, Technische Universität Berlin, Mueller-Breslau-Str. 8, 10623 Berlin, Germany, jonas.moeck@pi.tu-berlin.de), Lars Enghardt (DLR - German Aerospace Center, Mueller-Breslau-Str. 8, 10623 Berlin, Germany, lars.enghardt@dlr.de), Christian Oliver Paschereit (Institut für Strömungsmechanik und Technische Akustik, Technische Universität Berlin, Mueller-Breslau-Str. 8, 10623 Berlin, Germany, oliver.paschereit@tu-berlin.de)

To optimize the efficiency and to minimize the emissions of pollutants, modern combustion chambers for aeroengines or stationary gas turbines run in high temperature ranges with lean fuel-air mixtures. The disadvantage of this combustion mode is its susceptibility to the formation of thermoacoustic instabilities. These self-excited oscillations are an unacceptable noise source and, furthermore, they reduce the durability of the combustion chamber significantly. The focus of this paper is the localization of combustion noise sources in enclosed combustion chambers. This can be achieved using the acoustical nearfield information from pressure measurements with wall-flush mounted sensors. The spatially bounded region, where the sound sources are suspected, is discretized by a grid of monopole sources. A system of linear equations for the source strengths is built based on the relations between the sound sources and the sound pressure field, theoretically caused by the assumed sources of the grid. The source strengths are calculated by inverting the system of equations. A spatial variation of the source grid allows the scan of likely regions of sound sources to finally deduce their original distribution. Numerical simulation results will be shown to illustrate the potential and the limits of the proposed method.

\section{2:00}

3aPAc12. Computation of Indirect Combustion Noise by a CAA Method. Christoph Richter (Technische Universität Berlin, Sekr. MB1, Müller-Breslau-Str. 11, ISTA, 10623 Berlin, Germany, christoph.richter@tu-berlin.de), Frank H. Thiele (Technische Universität Berlin, Sekr. MB1, Müller-Breslau-Str. 11, ISTA, 10623 Berlin, Germany, Frank.Thiele@TU-Berlin.de)

Indirect combustion noise, which is generated by the acceleration of hot gases from a turbulent swirling flame, has become an important issue to understand turbo-machinery core noise. This indirect combustion noise it is dominated by a strong interaction of hydrodynamic and acoustic perturbations due to the transonic base flow in the turbine stages and combustion chamber exit nozzle. Current numerical simulation methods for the phenomenon based on compressible Large Eddy Simulation (LES) are very expansive. Thus LES applications are still limited to one design or few variations with immense computational costs. The most simple model fully covering indirect combustion noise, are the linearized non-isentropic Euler equations over an arbitrary mean flow. Therefore the gap between theory and LES is closed by a method adopted from Computational Aeroacoustics (CAA). It combines a high cost efficiency in terms of required points per wavelength with a high adaptability to realistic mean flow conditions. The CAA method is first validated with a simplified experiment and then applied to predict the acoustic properties of two model combustion chambers, one of them with transonic termination nozzle. By means of the acoustic intensity the sources of the noise are located.

\section{$12: 20$}

3aPAc13. Study of oscillating regimes in air-staged diffusion flames. Javier Ballester (University of Zaragoza / LITEC, Centro Politecnico Superior, Maria de Luna, 3, 50018 Zaragoza, Spain, ballester@unizar.es), Ricardo Hernández (University of Zaragoza / LITEC, Centro Politecnico Superior, Maria de Luna, 3, 50018 Zaragoza, Spain, richard@litec.csic.es), Ana Sanz (University of Zaragoza / LITEC, Centro Politecnico Superior, Maria de Luna, 3, 50018 Zaragoza, Spain, anasanz@litec.csic.es)

The occurrence of fluctuating behaviours in air-staged diffusion flames was studied experimentally in a gas-fired furnace. Burner settings (swirl levels, air distribution) were systematically varied, leading to a broad range of flame characteristics and pollutant emissions. Pressure fluctuations reached significant amplitudes and displayed characteristic frequencies for certain operating conditions. However, heat release fluctuations (as estimated from $\mathrm{OH}^{*}$ chemiluminescence) were correlated with pressure only in a part of those cases. Therefore, thermo-acoustic coupling is thought to exist only under certain conditions, whereas other oscillating regimes are not ascribed to acoustic feedback but to changes in the location of the flame stabilization region. This conclusion was supported by the values of the Rayleigh index calculated for the different regimes. An enhanced tendency to the onset of thermo-acoustic instabilities was observed for highly staged flames. 
3aPAc14. The acoustic wave propagation equation in a turbulent combusting flow. Jim B. Kok (University of Twente, P.O. Box 217, 7500 AE Enschede, Netherlands, j.b.w.kok@utwente.nl)

Sound generation by turbulent flames originates from the fluctuating heat release in the flame. The description of this fluctuating heat release and its effect on acoustics in turbulent flames is complicated due to the interaction of chemical reactions with turbulence, mixing and pressure fluctuations. In a turbulent flame the instantaneous density, velocity, pressure, temperature and species concentrations are determined by the transport equations for mass, momentum, enthalpy and species and by the equation of state. In the proposed paper an equation is formulated that describes the propagation of acoustic pressure fluctuations, and that determines the source terms. These source terms are compared with various terms derived in the literature. Significant differences are found between several approaches. An effort is made to come to a reliable and generally acceptable formulation. Subsequently events are ordered on basis of their typical time scale. That way source terms can be evaluated for the situation where the combustion is described with the use of time averaged chemical reaction progress variables and a mixture fraction variable. Subsequently the consequences of Reynolds and Favre averaging on these source terms and conservation of acoustical variables in a domain with turbulent flow are discussed.

WEDNESDAY MORNING, 2 JULY 2008

ROOM 362/363, 8:00 TO 9:20 A.M.

\title{
Session 3aPAd
}

\section{Physical Acoustics: Ultrasonics: Material Characterization I}

\author{
Bart Lipkens, Cochair \\ Western New England College, 1215 Wilbraham Road, Box S-5024, Springfield, MA 01119, USA \\ Nicolas Wilkie-Chancellier, Cochair \\ ECIME Université de Cergy-Pontoise, rue d'Eragny, Neuville-sur-Oise, 95031, France
}

\section{Contributed Papers}

\begin{abstract}
8:00
3aPAd1. Frequency sweeping and fluid flow effects on particle trajectories in ultrasonic standing waves. Bart Lipkens (Western New England College, 1215 Wilbraham Road, Box S-5024, Springfield, MA 01119, USA, blipkens@wnec.edu), Jason Dionne (Western New England College, 1215 Wilbraham Road, Box S-5024, Springfield, MA 01119, USA, jdionne@ flodesign.org), Michael Costolo (Physical Sciences Inc., 20 New England Business Center, Andover, MA 01810, USA, mac@psicorp .com), Edward Rietman (Physical Sciences Inc., 20 New England Business Center, Andover, MA 01810, USA, rietman@psicorp.com)
\end{abstract}

Particle concentration in ultrasonic standing waves by the acoustic radiation force is discussed. The acoustic radiation force is a function of the density and compressibility of the fluid and the suspended particles. A twodimensional theoretical model is developed for particle trajectory calculations. An electro-acoustic model is used to predict the acoustic field in a resonator. The results of the linear acoustic model are used to calculate the acoustic radiation force acting on a particle suspended in the resonator. A particle trajectory model is developed that integrates the equation of motion of a particle subjected to a buoyancy force, a fluid drag force, and the acoustic radiation force. Computational fluid dynamics calculations are performed to calculate the velocity field. For a fixed frequency excitation, the particles are concentrated along the stable node locations of the acoustic radiation force. Through a periodic sweeping of the excitation frequency particle translation is achieved. Two types of frequency sweeps are considered, a ramp approach and a step-change method. Numerical results of particle trajectory calculations in a resonator with dimensions much larger than a typical wavelength are presented. A movie of experimental observations of particle trajectories is shown to illustrate the method.

\section{8:20}

3aPAd2. Elastic constants identification of anisotropic composite rectangular parallelepipeds. Thomas Delaunay (Université Montpellier II, Place Eugène Bataillon, 34095 Montpellier, France, delaunay@lain .univ-montp2.fr), Didier Laux (Université Montpellier II, Place Eugène Bataillon, 34095 Montpellier, France, laux@lain.univ-montp2
.fr),Jean-Yves Ferrandis(Radio Application Division, NEC Corporation, Université Montpellier II, Place Eugène Bataillon, 34095 Montpellier, France, ferrandi@lain.univ-montp2.fr)

For many years, composite materials have been investigated due to their potential in many applications such as nuclear fuel. Nowadays, the improvement of structure conception and simulation requires many reliable properties, especially the entire elastic tensor. For these reasons, the implementation of simulation and characterization methods adapted to the geometry of samples and the high anisotropy degree of such materials are then crucial issues to developers and end users. The work presented here reports first macroscopic properties of two composites Duralumin/Air and Duralumin/Tungsten Carbide) calculated using periodical homogenization method from elastic constants of each phase measured by high frequency acoustic microscopy. Then a resonant ultrasonic spectroscopy method is used to identify the entire elastic tensor. First, computation predicting the resonant frequencies and the mode shapes of free vibrations of cubes are presented. Excitation is then ensured by ultrasonic transducers and detection is performed by means of a laser vibrometer. A fit process of the theoretical frequencies on the measured data is then described. This method allows us to conclude about the accuracy of the homogenization method.

\section{8:40}

3aPAd3. Viscoelastic material monitoring using an acoustical cylindrical spectrometer. Nicolas Wilkie-Chancellier (ECIME Université de Cergy-Pontoise, rue d'Eragny, 95031 Neuville-sur-Oise, France, Nicolas.Wilkie-Chancellier@u-cergy.fr), Loic Martinez (ECIME Université de Cergy-Pontoise, rue d'Eragny, 95031 Neuville-sur-Oise, France, loic.martinez@u-cergy.fr), Pascal Griesmar (ECIME Université de CergyPontoise, rue d'Eragny, 95031 Neuville-sur-Oise, France, pascal.griesmar @u-cergy.fr), Stéphane Serfaty (ECIME Université de Cergy-Pontoise, rue d’Eragny, 95031 Neuville-sur-Oise, France, stephane.serfaty@u-cergy.fr)

Acoustic sensors often have a plane geometry due to manufacturing constrains. In the present work, the cylindrical geometry is investigated with a normal incident beam in order to suppress the edge effects on mode conversion. The experimental setup uses an aluminium cylindrical shell. A 
classical immersion transducer ( $2 \mathrm{MHz}$ central frequency) sends a plane pulse wave on the shell at normal incidence. A second immersion transducer can turn around, enabling the observation of the waves scattered by the cylindrical shell. Previous works have shown that the acoustic field scattered by the shell as a rainbow structure enabling the acoustic spectrometry of the material poured inside the shell. From these results, the present study proposes an acoustical model linking the resonances parameters to the material ones, enabling the monitoring of time varying viscous materials. Several gelation monitoring experiments are performed and analysed using the proposed model. Results are in good agreement with quartz sensor measurements and offer a good alternative to wide frequency range measurements.

\section{9:00}

3aPAd4. Ultrasonic and photoacopustic investigations of water mixtures with dioxanes 1-3 \& 1-4. Bogumil B. Linde (Inst. of Exper. Physics, Univ. of Gdańsk, Ul. Wita Stwosza, Bldg. 57, 80-952 Gdańsk, Poland, fizbl@univ.gda.pl), Anna K. Kiszel (Inst. of Exper. Physics, Univ. of
Gdańsk, Ul. Wita Stwosza, Bldg. 57, 80-952 Gdańsk, Poland, ania_tczew84 @wp.pl), Anna Sikorska (Inst. of Exper. Physics, Univ. of Gdańsk, U1. Wita Stwosza, Bldg. 57, 80-952 Gdańsk, Poland, fizams@univ.gda.pl)

Ultrasonic velocities, densities and thermal effusivity dependence on concentration were measured in the mixtures of water with dioxanes 1-3 and $1-4$ in the temperature range from 291.15 to $303.15 \mathrm{~K}$. Adiabatic compressibilities were calculated from Laplace's equation based on the experimental results obtained. Variations of these values with concentration and temperature were studied. Structural interactions and the formation of a compact pseudo-stable structure at very low concentrations of these liquids were observed. The plots of the adiabatic compressibility versus the mole fraction of these molecules display two characteristic points at low concentrations: the intersection of the isotherms and their minimum. Such relations between adiabatic compressibility, concentration and temperature are usually attributed to the formation of pseudo-stable molecular structures. To formulate a model of local structures present in the investigated molecular systems it is indispensable to get an insight into hydration of molecules and the formation of hydrogen bonds. Therefore, the attention was focused particularly on these problems.

WEDNESDAY MORNING, 2 JULY 2008

ROOM 362/363, 9:20 A.M. TO 12:40 P.M.

\title{
Session 3aPAe
}

\section{Physical Acoustics: Ultrasonics: NDT and Layered Systems I}

\author{
Vincent Pagneux, Cochair \\ Laboratoire d'Acoustique de l'Université du Maine, UMR CNRS 6613, AV. O. Messiaen, Le Mans, 72085, France \\ Claire Prada, Cochair \\ Laboratoire Ondes et Acoustique, ESPCI, Université Paris 7, CNRS, 10 rue Vauquelin, Paris, 75005, France
}

\section{Contributed Papers}

\section{9:20}

3aPAe1. Thickness determination of a multilayered system of different materials by natural frequencies. Changzhi Zhou (Institute of Acoustics, Chinese Academy of Sciences, NO.21, Bei-Si-huan-Xi Road, 100080 Beijing, China, zcz@mail.ioa.ac.cn), Mingxuan Li (Institute of Acoustics, Chinese Academy of Sciences, NO.21, Bei-Si-huan-Xi Road, 100080 Beijing, China, 1mx@mail.ioa.ac.cn), Jie Mao (Institute of Acoustics, Chinese Academy of Sciences, NO.21, Bei-Si-huan-Xi Road, 100080 Beijing, China, maojie@mail.ioa.ac.cn), Xiaomin Wang (Institute of Acoustics, Chinese Academy of Sciences, NO.21, Bei-Si-huan-Xi Road, 100080 Beijing, China, wangxiaomin@mail.ioa.ac.cn)

This paper is focusing on the relationship between the natural frequencies of a multilayered system of different isotropic elastic materials and the thickness of each layer. A relevant ultrasonic method for thickness determination of a multilayered system by natural frequencies has been developed. The natural frequencies are numerically calculated from the normally incident reflection coefficient of a "steel-epoxy resin-aluminum-thin polymer" structured lamination. Some natural frequencies are sensitive to one layer thickness in certain range while the others are invariable. This phenomenon is explained by the defined strain energy ratio, and used for our thickness inversion. The measured natural frequencies of a specimen show good agreement with the theoretical ones. All the natural frequencies are taken into account, and the simulated annealing method is employed for the thickness inverse calculation of the multilayered structure. The average relative errors of calculated thickness of the each layer are $-0.7 \%, 35 \%,-1.3 \%$ and $-6.1 \%$, corresponding to the real values: $1957 \mu \mathrm{m}, 16 \mu \mathrm{m}, 1913 \mu \mathrm{m}$ and $54 \mu \mathrm{m}$.
9:40
3aPAe2. Bonding quality evaluation of layered materials by guided wave dispersion. Young H. Kim (Korea Science Academy, 111 Backyangkwanmoonro, Busanjin-ku, 614-822 Busan, Republic of Korea, youngkim@paran.com), Minseok Bae (Korea Science Academy, 111 Backyangkwanmoonro, Busanjin-ku, 614-822 Busan, Republic of Korea, bms1991@empal.com), Sunhee Choi (Korea Science Academy, 111 Backyangkwanmoonro, Busanjin-ku, 614-822 Busan, Republic of Korea, cjtwo120@hanmail.net), Jisu Kim (Korea Science Academy, 111 Backyangkwanmoonro, Busanjin-ku, 614-822 Busan, Republic of Korea, boone90@hotmail.com), Seokchan Hong (Korea Science Academy, 111 Backyangkwanmoonro, Busanjin-ku, 614-822 Busan, Republic of Korea, charliehongn@hanmail.net)

Multi-layered materials have been widely used for high performance materials with advanced properties required for the critical parts of structures. The characteristics of bonding quality in multi-layered materials have been hot issue for a long time. The bonding quality was evaluated by using dispersion of guided wave. Broadband guided waves were generated at oblique incidence angle, and waves reflected at the edge of the specimen were detected by using a pulse-echo setup. The dispersion curves were obtained from edge-reflected leaky guided wave for various plates: Single layered plates of aluminum, copper and stainless steel, and double layered plates of aluminum-stainless steel cladding, epoxy-bonded aluminumstainless and epoxy-bonded aluminum-copper. The aluminum-stainless steel cladding specimen showed similar dispersion for both sides, whereas epoxybonded specimens showed different dispersions which are similar to that of upper layer only. These results imply that the cladding specimen used in the 
present work is well bonded, whereas epoxy-bonded specimens are acoustically isolated. The dispersion curve of guided wave has high potential to evaluate bonding quality of layered materials.

\section{0:00}

3aPAe3. Acoustical imaging with bending waves based on sparse inversion. Lars Hörchens (University of Technology Delft, Lorentzweg 1, 2628 CJ Delft, Netherlands, 1.horchens@tudelft.nl), Diemer De Vries (University of Technology Delft, Lorentzweg 1, 2628 CJ Delft, Netherlands,d.devries@tudelft.nl)

Bending waves can be employed in the context of acoustical imaging for the detection of material defects or localization of vibration sources. The obtainable spatial resolution is limited by the minimal wavelength contained in the excitation signal. The evanescent part of the wave field can usually not be used for the imaging of sources at distances exceeding a wavelength Therefore only the propagating part of the field can be employed, which means that the minimum size of the region where energy can be focused is in the order of half a wavelength due to the diffraction limit. If the recovery of vibration sources and reflections is posed as an inverse problem, regularization techniques can be applied in order to force a sparse solution. Preliminary results indicate that the assumption of a sparse source distribution can be used to resolve features significantly smaller than half a wavelength. The approach is therefore applied as a high-resolution imaging technique for bending wave fields.

\section{0:20-10:40 Break}

\section{0:40}

3aPAe4. An experimental method of corrugated surface reconstruction investigated in combination with finite-element simulation. Sarah W. Herbison (Georgia Tech Lorraine - G.W. Woodruff School of ME, UMI Georgia Tech - CNRS 2958, 2 rue Marconi, 57070 Metz, France, sherbison@gatech.edu), Ebrahim Lamkanfi (Georgia Tech Lorraine - G.W. Woodruff School of ME, UMI Georgia Tech - CNRS 2958, 2 rue Marconi, 57070 Metz, France, Ebrahim.Lamkanfi@ugent.be), Nico F. Declercq (Georgia Tech Lorraine - G.W. Woodruff School of ME, UMI Georgia Tech - CNRS 2958, 2 rue Marconi, 57070 Metz, France, nico .declercq@me.gatech.edu)

Current theory applied to ultrasonic diffraction from periodically corrugated surfaces is valid only in the far-field; it cannot be applied to determine the diffracted field within the surface corrugations themselves. Therefore, information regarding the corrugation is difficult to extract from experiments that are necessarily conducted in the far-field. The present work aims to simulate a potential experimental method for investigating the corrugations themselves. Using near-field results obtained from a finite-element code, the far-field diffraction is constructed as a series of time-harmonic plane waves. By back-propagating this diffracted field, information about the corrugations is obtained. Comparing the results of the back-propagated field to the field generated by the finite-element code shows the limitations of determining details of periodically corrugated surfaces from far-field experiments.

\section{1:00}

3aPAe5. Determination of the width of an axisymmetric deposit on a metallic pipe by means of Lamb type guided modes. Mustapha El Moussaoui (LOMC FRE 3102 CNRS Groupes Ondes Acoustiques, Université du Havre (IUT), Place Robert Schuman, 76610 Le Havre, France, elmoussm@univ-lehavre.fr), Farid Chati (LOMC FRE 3102 CNRS Groupes Ondes Acoustiques, Université du Havre (IUT), Place Robert Schuman, 76610 Le Havre, France, farid.chati@univ-lehavre.fr), Fernand Leon (LOMC FRE 3102 CNRS Groupes Ondes Acoustiques, Université du Havre (IUT), Place Robert Schuman, 76610 Le Havre, France, fernand.leon@univ-lehavre.fr), Aleksander Klauson (Tallinn University of Technology, Dept. of Mechanics, Ehitajate tee 5, 19086 Tallinn, Estonia,
aklauson@staff.ttu.ee),Gerard Maze(LAUE, Université du Havre, Place Robert Schuman, F-76610 Le Havre, France, gerard.maze@univ-lehavre.fr)

The non-destructive evaluation of pipes using guided waves is extensively used for the detection and the size estimation of defects. The important number of investigations concerning with the interaction between a guided wave and a defect show the growing need to improve our knowledge of this phenomenon in order to detect and to characterize as well as possible the defect. This work is devoted to the evaluation of the width of an axisymmetric resin deposit coupled to the wall of a metallic tube by means of longitudinal modes S0, S1. To that end, we interest to damping of these modes provoked by the deposit. The study is carried out both from the numerical point of view and from the experimental point of view in considering a deposit of variable width. The finite element method is employed to model the propagation and the diffraction of Lamb-type waves. The experiment is based on the generation of axisymmetric waves on the section of a tube by a piezoelectric transducer. Measurements of temporal echoes reflected by the deposit and transmitted after the deposit are achieved. A mathematical law of the attenuation as function of the width is deduced for each wave.

\section{$11: 20$}

3aPAe6. Scattering by a defect in an elastic waveguide: Coupling of finite elements and modal representations. Vahan Baronian (CNRS, ENSTA - 32 Boulevard Victor, 75015 Paris, France, vahan.baronian @ensta.fr), Anne-Sophie Bonnet- Ben Dhia (CNRS, ENSTA - 32 Boulevard Victor, 75015 Paris, France, bonnet@ensta.fr), Colin Chambeyron (CNRS, ENSTA - 32 Boulevard Victor, 75015 Paris, France, colin.chambeyron@ensta.fr), Eric Luneville (ENSTA - CEA, 32 Boulevard Victor, 75015 Paris, France, eric.luneville@ensta.fr)

The potentiality of guided ultrasonic waves in nondestructive testing is currently investigated. Indeed, guided waves can allow a rapid inspection of large areas and of non accessible parts of particular structures like plates or pipes, compared to conventional techniques. This work concerns the numerical finite element computation, in the frequency domain, of the diffracted wave produced by a defect (crack, heterogeneity, discontinuity, local bend etc.) located in an infinite elastic waveguide. The computational domain is chosen as a portion of the waveguide, containing the perturbation, and our purpose is to build transparent conditions on its artificial boundaries by using modal representations. This cannot be achieved in a classical way, due to nonstandard properties of elastic modes. However, a biorthogonality relation is established which allows to derive a transparent condition relating hybrids displacement/stress vectors. An original mixed formulation is then implemented, whose unknowns are the displacement field in the bounded domain and the normal component of the normal stresses on the artificial boundaries. Numerical validations are presented in the two-dimensional and three-dimensional cases.

\section{$11: 40$}

3aPAe7. Interaction of elastic waves with dislocations. Agnes Maurel (LOA/ESPCI, 10 rue Vauquelin, 75005 Paris, France, agnes.maurel@espci.fr), Vincent Pagneux (Laboratoire d'Acoustique de l'Université du Maine, UMR CNRS 6613, AV. O. Messiaen, 72085 Le Mans, France, vincent.pagneux@univ-lemans.fr), Felipe Barra (Universidad de Chile, av. Blanco encalada, 0 Santiago, Chile, fbarra@uchile.cl), Fernando Lund (CIMAT, Universidad de Chile, 0 Santiago,Chile, flund@cimat.cl)

This work concerns the effective propagation of elastic waves in a medium filled with randomly placed dislocations. Multiple scattering theory is applied to derive the effective velocity and attenuation of the waves (both transverse and longitudinal) because of the effect of the dislocations. Our work is a generalization of Granato \& Lucke theory since it takes into account the polarization of the elastic waves. Two principal results have been obtained, in connexion with experiments: 1) we have explained the different attenuations experimentally measured between longitudinal and transverse waves. The difference between the two values is obtained in quantitative agreement with no adjustable parameter. 2) we have offered a scenario for the frequency law in the attenuation measured in polycrystals (a combina- 
tion of quadratic and quartic law). This has been done by taking into account the dislocations at the grain boundaries that can contribute to the attenuation process.

\section{2:00}

3aPAe8. Particle deposit formation during filtration characterisation using ultrasonic waves. Didier Laux (Université Montpellier II, Place Eugène Bataillon, $34095 \quad$ Montpellier, France, laux@lain .univ-montp2.fr), Hugues Blasco (Université Montpellier II, Place Eugène Bataillon, 34095 Montpellier, France, blasco@lain.univ-montp2 .fr), Jean-Yves Ferrandis (Radio Application Division, NEC Corporation, Université Montpellier II, Place Eugène Bataillon, 34095 Montpellier, France, ferrandi@lain.univ-montp2.fr), Julie Mendret (INSA Toulouse, Av de Rangueil, 31077 Toulouse, France, julie.mendret@insa-toulouse .fr), Christelle Guigui (INSA Toulouse, Av de Rangueil, 31077 Toulouse, France, guigui@insa-toulouse.fr), Corinne Cabassud (INSA Toulouse, Av de Rangueil, 31077 Toulouse, France, cabassub@insa-toulouse.fr), Philippe Schmitz (INSA Toulouse, Av de Rangueil, 31077 Toulouse, France, philippe.schmitz@insa-toulouse.fr)

Membranes filtration processes are widely used because of their ability to remove particles, colloidal species and micro-organisms from different liquids feeds. However an inherent process limitation is the membrane fouling due to deposition of suspended matter during filtration. Therefore the understanding of formation and transport properties of particle deposit responsible for membrane fouling is a necessary step to optimize membrane processes. These deposits are non homogeneous, highly porous and very thin (less than $500 \mu \mathrm{m}$ ). Thus, it is necessary to obtain local information in order to analyze and model the basic mechanisms involved in deposit formation and then to further predict process operation. As local parameters such as cake thickness and porosity are hardly reachable with conventional techniques, we propose in this paper the use of an ultrasonic echographic method. In a first step this method is validated on deposits of small glass balls. We show that the porosity and thickness of the deposit is in good agreement with theory. Then, the ultrasonic technique has been adapted on a filtration cell in order to give in line results during filtration. First results are presented and discussed.

\section{2:20}

3aPAe9. Single-sided air-coupled ultrasonic NDT of thick plates. Steven Delrue (K.U.Leuven Campus Kortrijk, E. Sabbelaan 53, 8500 Kortrijk, Belgium, steven.delrue@kuleuven-kortrijk.be), Pieter Lust (Katholieke Hogeschool Zuid-West-Vlaanderen dept. VHTI, Doorniksesteenweg 145, 8500 Kortrijk, Belgium, pieter.lust@katho .be), Jurgen Deveugele (Katholieke Hogeschool Zuid-West-Vlaanderen dept. VHTI, Doorniksesteenweg 145, 8500 Kortrijk, Belgium, jurgen.deveugele@katho.be), Erik Blomme (Katholieke Hogeschool ZuidWest-Vlaanderen dept. VHTI, Doorniksesteenweg 145, 8500 Kortrijk, Belgium, Erik.Blomme@katho.be), Olivier Bou Matar (IEMN, UMR CNRS 8520, avenue Poincaré, BP 60069, 59652 Villeneuve d'Ascq, France, olivier.boumatar@iemn.univ-lille1.fr), Koen Van Den Abeele (K.U.Leuven Campus Kortrijk, E. Sabbelaan 53, 8500 Kortrijk, Belgium, koen .vandenabeele@kuleuven-kortrijk.be)

Non-contact air-coupled ultrasonic inspection using single sided access to the material offers interesting possibilities for the development of ultrasonic in-line NDT-systems. This contribution reports observations and simulations obtained from a single-sided air-coupled pitch-catch configuration. The feasibility of the technique is demonstrated with respect to artificial defects in aluminium samples. Depending on the configuration it is possible to establish one or more ultrasonic images of the defect, their number and relative position containing information about the location and depth of the defect. The experiments are simulated using a spectral solution obtained within Comsol, and compared with a full 2D Finite Difference Time Domain simulation and a ray tracing (shadow) approach. The simulations allow simple prediction of the response images in more complicated experimental conditions.

WEDNESDAY MORNING, 2 JULY 2008

ROOM 351, 11:20 A.M. TO 12:40 P.M.

\title{
Session 3aPAf
}

\section{Physical Acoustics: Ultrasonics: Industrial NDT I}

\author{
Bertrand Nongaillard, Cochair \\ IEMN - DOAE, Université de Valenciennes, Le Mont - Houy, Valenciennes, 59313, France \\ Christophe Aristegui, Cochair \\ LMP, UMR CNRS 5469, Université Bordeaux I, 351, cours de la Libération, Talence, 33405, France
}

\section{Contributed Papers}

\section{1:20}

3aPAf1. Simple and versatile non-contact technique for measuring the interfacial tension of a liquid-liquid interface using pulsed acoustic radiation pressure. Bruno Issenmann (Bordeaux University - $\mathrm{CPMOH}$ 351 cours de la Liberation, 33405 Talence Cedex, France, b.issenmann @ cpmoh.u-bordeaux1.fr), Regis Wunenburger (Bordeaux University - CP$\mathrm{MOH}, 351$ cours de la Liberation, 33405 Talence Cedex, France, r.wunenburger@cpmoh.u-bordeaux1.fr), Jean-Pierre Delville (Bordeaux University - CPMOH, 351 cours de la Liberation, 33405 Talence Cedex, France, jp.delville@cpmoh.u-bordeaux1.fr)

We present a versatile non-contact technique for measuring the interfacial tension of a liquid-liquid interface using the acoustic radiation pressure. This technique is based on the analysis of the shape of the time-dependent deformation of the liquid interface induced by the radiation pressure of a focused, pulsed ultrasonic beam. It combines a simple optical detection step and a novel analytical model of interface deformation dynamics that accurately takes into account interfacial, gravitational, as well as viscous effects in both weakly and highly viscous limits. The accuracy of this technique is experimentally demonstrated on liquid-air and liquid-liquid interfaces.

\section{1:40}

3aPAf2. Diffuse ultrasonic backscatter at oblique incidence for heterogeneous solids. Joseph A. Turner (University of Nebraska, Dept. of Engineering Mechanics, W317.4 NH, Lincoln, NE 68588, USA, jaturner@unl.edu), Goutam Ghoshal (University of Nebraska, Dept. of Engineering Mechanics, W317.4 NH, Lincoln, NE 68588, USA, goutamghoshal@rediffmail.com)

Diffuse ultrasonic backscatter techniques are useful for probing heterogeneous materials. They can be used to extract microstructural parameters and to detect flaws which cannot be detected by conventional ultrasonic 
techniques. Such experiments, usually done using a modified pulse-echo technique, utilize the spatial variance of the signals as a primary measure of microstructure. Quantitative ultrasonic scattering models include components of both transducer beams as well as microstructural scattering information. Of particular interest for interpretation of many experiments is the propagation through a liquid-solid interface at normal and oblique incidence. Here, the Wigner distribution of the beam pattern of an ultrasonic transducer through a liquid-solid interface is used in conjunction with the stochastic wave equation to model this scattering problem within a single scattering formulism. The Wigner distribution represents a distribution in space and time of the spectral energy density as a function of wave vector and frequency. A Gaussian beam is used to model the transducer beam pattern. The scattered response in the time domain is then compared with experimental results for materials of common interest. These results are anticipated to impact ultrasonic nondestructive evaluation and characterization of heterogeneous media.

\section{Invited Paper}

\section{2:00}

3aPAf3. Model-based reconstruction methods of NDT ultrasonic array data. Pierre Calmon (CEA LIST, Centre de Saclay, F-91191 Gif sur Yvette, France, pierre.calmon@cea.fr), Ekaterina Iakovleva (CEA LIST, Centre de Saclay, F-91191 Gif sur Yvette, France, ekaterina.iakovleva@cea.fr), Alex Fidahoussen (CEA LIST, Centre de Saclay, F-91191 Gif sur Yvette, France, alex.fidahoussen@cea.fr), Guillemette Ribay (CEA LIST, Centre de Saclay, F-91191 Gif sur Yvette, France, guillemette.ribay@cea.fr)

Ultrasonic array techniques are more and more used in NDT applications because of their flexibility and of their adaptability to complex configurations. In addition they can provide richer data than conventional methods. An optimal exploitation of such data in the aim of localizing and characterizing the detected flaws requires the application of imaging and reconstruction techniques based on a direct modeling of the propagation of ultrasound from the transmitter to the receiver. The quantitative models for the propagation which are available in the CIVA software platform developed at CEA-LIST allow such direct modelling in parts of possibly complex geometries and materials. Different array data reconstruction methods based on the exploitation of these direct models have been studied and implemented in the plat-form. Thus, in this communication we present in particular algorithms derived of synthetic focusing approach. These algorithms exploit the time of flight identification and can be apply to any set of array data. We also present a reconstruction method based on the post-processing of the transfert matrix of the array and the application of MUSIC algorithm. We show and discuss results obtained with these methods both on simulated and experimental data.

\section{Contributed Paper}

12:20

3aPAf4. Low frequency composite acoustic sensor for highly absorbing media characterization. Alain Skaf (IEMN - DOAE, Université de Valenciennes, Le Mont - Houy, 59313 Valenciennes, France, alain.skaf@univ -valenciennes.fr), Georges Nassar (IEMN - DOAE, Université de Valenciennes, Le Mont - Houy, 59313 Valenciennes, France, gnassar@univvalenciennes.fr), Fabrice Lefebvre (IEMN - DOAE, Université de Valenciennes, Le Mont - Houy, 59313 Valenciennes, France, Fabrice.Lefebvre @univ-valenciennes.fr), Bertrand Nongaillard (IEMN - DOAE, Université de Valenciennes, Le Mont - Houy, 59313 Valenciennes, France, Bertrand .Nongaillard@univ-valenciennes.fr)

Ultrasonic techniques are widely used in nondestructive testing and evaluation of media. But when media are highly absorbing, it becomes often impossible to operate at classical ultrasonic frequencies. In this work, we propose a low frequency acoustic device to characterize such highly absorbing media. This device is composed of a piezoelectric disc embedded in a metallic ring. The technique consists in bringing into resonance the entire structure. Firstly, we will study analytically and numerically the main resonance modes of the sensor, which are the flexion modes and the radial modes. The results of the modelization are compared to measurements obtained through an impedance analyzer. In a second part, we will use this model of sensor to characterize two different media. The first type consists of a series of polymer disks with different viscoelastic properties. Using radial modes of vibration, we will characterize these polymer disks through measurement of the acoustic velocity. After that, we will characterize using the flexion modes a very complex medium which present a highly absorbing phenomenon such as dough in the fermentation phase. The measurements show the aptitude of this technique to follow the evolution of the physical properties of complex media. 


\title{
Session 3aPPa
}

\section{Psychological and Physiological Acoustics and ASA Committee on Standards: Applications of Psychoacoustics III}

\author{
Patricia Davies, Cochair \\ Ray W. Herrick Lab., School of Mechanical Engineering, Purdue Univ., 140 S. Martin Jischke Drive, West Lafayette, IN \\ 47907-2031, USA \\ Hugo Fastl, Cochair \\ AG Technische Akustik, MMK, TU München, Arcisstr. 21, München, 80333, Germany
}

\section{Contributed Papers}

\begin{abstract}
8:00
3aPPa1. Psychoacoustic evaluation of compression drives. Joan Cruanes Catala (Escola Politècnica Superior de Gandia, Universitat Politècnica de València, Crtra Natzaret-Oliva s/n, 46730 Gandia, Spain, joacrca@upvnet.upv.es), Jorge Frances Monllor (DFISTS. Univ. de Alicante, Carretera de Sant Vicent del Raspeig s/n, 03690 San Vicente del Raspeig, Spain, jfmonllor@ua.es), Jaime Ramis Soriano (DFISTS. Univ. de Alicante, Carretera de Sant Vicent del Raspeig s/n, 03690 San Vicente del Raspeig, Spain, jramis@ua.es), Jesús Alba Fernandez (Escola Politècnica Superior de Gandia, Universitat Politècnica de València, Crtra NatzaretOliva s/n, 46730 Gandia, Spain, jesalba@fis.upv.es)

The following work tries to be an approximation to the sound quality valuation of radiant systems, in concrete, the case of compression drives. This approach is realized from different perspectives: on one hand, an evaluation of the quality of these systems from the point of view of the psychoacoustic classic parameters described by Zwicker will be done. On the other hand, a perceptual model described by Moore and based on the human auditory system will be applied. Both models will be applied in signals registered on different commercial models of compression drives. The work also includes the accomplishment of a psychoacoustic experiment in which a hearing will realize a valuation of perceived quality. Finally, one will try to establish a correlation with the information obtained in the three mentioned approaches.
\end{abstract}

\section{$8: 20$}

3aPPa2. A study of evaluating the button sounds. Shunsuke Ishimitsu (Hiroshima City University / National Institute of Advanced Industrial Science and Technology, 3-4-1 Ozuka-Higashi, Asa-Minami-Ku, 731-3194 Hiroshima, Japan, ishimitu@hiroshima-cu.ac.jp), Koji Sakamoto (Hiroshima City University, 3-4-1 Ozuka-Higashi, Asa-MinamiKu, 731-3194 Hiroshima, Japan, koji_s@tm.ten.fujitsu.com), Takayuki Arai (Univ. of Hyogo, 2167, Shosha, 671-2280 Himeji, Japan, es07g001 @ steng.u-hyogo.ac.jp), Toshikazu Yoshimi (Pioneer Corp., 25-1, Nisimachi, Yamada, 350-8555 Kawagoe, Japan, toshikazu_yoshimi@post .pioneer.co.jp), Yuichi Fujimoto (Pioneer Corp., 25-1, Nisimachi, Yamada, 350-8555 Kawagoe, Japan, yuichi_fujimoto@post.pioneer.co.jp), Kenichi Kawasaki (Pioneer Corp., 25-1, Nisimachi, Yamada, 350-8555 Kawagoe, Japan, kenichi_kawasaki@post.pioneer.co.jp)
In recent years, much attention has been directed at the sound design which designs various sound treated as noise, such as automobile acceleration sound and cleaner sound, because the point of view sound is a part of products, has permeated. This research considered the sound design and its evaluation about 11 kinds of the button sounds. First, the impression was extracted by the SD method and relevance with time frequency analysis was investigated. Moreover, we also confirmed that an impression changed, when the sound of a bad impression is processed into the sound of a good impression using adaptive control.

\section{8:40}

3aPPa3. Factoring end-user expectations into the sound design process of computer keyboards. Romain Boulandet (Ecole Polytechnique Fédérale de Lausanne, EPFL STI LEMA, Station 11, CH 1015 Lausanne, Switzerland, romain.boulandet@epfl.ch), Hervé Lissek (Ecole Polytechnique Fédérale de Lausanne, EPFL STI LEMA, Station 11, CH 1015 Lausanne, Switzerland, herve.lissek@epfl.ch), Sylvain Sauvage (Logitech Europe S.A., Z.I. Moulin du choc D, CH 1122 Romanel/Morges, Switzerland, sylvain_sauvage@eu.logitech.com), Jacques Robert (Logitech Europe S.A., Z.I. Moulin du choc D, CH 1122 Romanel/Morges, Switzerland, jacques_robert@eu.logitech.com)

The poor acoustic comfort of usual commercial products is often perceived as annoying. Today, more and more companies have realized that sound can be an important component of product attributes and image. They are interested in having acoustic guidelines for enhancing their products sound quality. The objective of this research is to quantify the objective and subjective noise production of computer keyboards, and hence to translate the pleasantness in an objective way. Our strategy consists in developing both benchmarks and listening tests to ensure that sound design process never looses sight of the end-users expectations. The presented work proposes an original methodology to define what keystroke sound matches the image of a pleasant keyboard. We also approach what acoustic characteristics have a significant influence on the global appreciation of keystroke sounds. Good results have been obtained showing a strong correlation between perceptive cues and metrics. 


\title{
Session 3aPPb
}

\section{Psychological and Physiological Acoustics: Auditory Perception of Sound Source Properties I}

\author{
John Neuhoff, Cochair \\ The College of Wooster, 930 College Mall, Wooster, OH 44691, USA \\ Anna Preis, Cochair \\ Institute of Acoustics, Adam Mickiewicz University, Unultowska 85, Poznan, 61-614, Poland \\ Invited Paper \\ 8:00
}

\begin{abstract}
3aPPb1. Non-visual identification of walking grounds. Bruno L. Giordano (Centre for Interdisciplinary Research in Music Media \& Technology (CIRMMT) - Schulich School of Music - McGill Univ., 555 Sherbrooke Street West, Montreal, QC H3A1E3, Canada, bruno.giordano@music.mcgill.ca), Stephen McAdams (Centre for Interdisciplinary Research in Music Media \& Technology (CIRMMT) - Schulich School of Music - McGill Univ., 555 Sherbrooke Street West, Montreal, QC H3A1E3, Canada, smc@music.mcgill.ca), Yon Visell (Centre for Interdisciplinary Research in Music Media \& Technology (CIRMMT) - Schulich School of Music - McGill Univ., 555 Sherbrooke Street West, Montreal, QC H3A1E3, Canada, yon@cim.mcgill.ca), Jeremy Cooperstock (Centre for Intelligent Machines - CIRMMT - Dept. of Electrical and Computer Engineering - McGill Univ., 3480 University Street, Montreal, QC H3A 2A7, Canada, jer@cim.mcgill.ca), Hsin-Yun Yao (Centre for Intelligent Machines - McGill Univ., 3480 University Street, Montreal, QC H3A 2A7, Canada, hyyao@cim.mcgill.ca), Vincent Hayward (Centre for Intelligent Machines - McGill Univ., 3480 University Street, Montreal, QC H3A 2A7, Canada, hayward@cim.mcgill.ca)
\end{abstract}

We investigated the role of haptic, proprioceptive and auditory information in the non-visual identification of walking grounds. We selected four solid materials (e.g., marble) and four aggregate materials (e.g., fine gravel). Five observers identified the materials in each of four experimental conditions: multisensory, haptic, proprioceptive, and auditory. In the auditory condition, they were presented with walking sounds they produced. In the other conditions, observers walked blindfolded on the materials. In the haptic and proprioceptive conditions auditory information was masked. In the proprioceptive condition haptic information was masked. No masking took place in the multisensory condition. In all conditions, solids and aggregates were seldom confused, and aggregates were better identified than solids. Chance identification performance was observed only for solids in the presence of simultaneous haptic and auditory masking, suggesting a secondary role of proprioceptive information. In the proprioceptive condition, identification of aggregates was better than chance, suggesting a significant role of proprioception. Identification of aggregates was at its best in the haptic condition, and not in the multisensory condition, suggesting either an inconsistency of information between auditory and non-auditory channels, or conflicting cognitive strategies for the exploitation of these sources of information in multisensory contexts.

\section{Contributed Paper}

8:20

3aPPb2. The effect of tone envelope on sensory integration: support for the 'unity assumption'. Michael Schutz (University of Virginia, 102 Gilmer Hall, Charlottesville, VA 22904, USA, schutz@ virginia .edu), Michael Kubovy (University of Virginia, 102 Gilmer Hall, Charlottesville, VA 22904, USA, kubovy@virginia.edu)

Research on the integration of auditory and visual sensory information consistently confirms the optimal integration hypothesis, according to which information is weighted according to its relative quality. Thus, since the auditory system has greater temporal resolution, this hypothesis predicts that visual information will not affect auditory judgments of duration. In conflict with this hypothesis, Schutz \& Lipscomb (2007) report that percussionists use visual information to alter audience perception of note duration. To show that this discrepancy arises from the acoustic characteristics of percussive sounds, we paired visual information derived from a striking motion with pure tones exhibiting two types of envelopes: percussive (sharp onset followed by exponential decay) and flat (sharp onset to a fixed level, followed by sharp offset) envelopes. Visual information affected auditory duration judgments only for the tones with percussive envelopes (e.g. the sounds produced by a striking motion). As ratings of percussive tones were no more variable than ratings of flat tones, the difference cannot be explained by "ambiguity" of percussive-tone duration. In contrast to the notion of optimal integration, we conclude that envelope is an important acoustic cue for cross-modal integration and offers important information regarding event identification.

\section{Invited Papers}

\section{8:40}

3aPPb3. Subjective verification of simulation of a vehicle pass-by. Tomasz Kaczmarek (Institute of Acoustics, Adam Mickiewicz University, Unultowska 85, 61-614 Poznan, Poland, tomek@spl.ia.amu.edu.pl)

The simulation of a moving vehicle can be a very powerful tool for investigating the perception of vehicle motion (velocity, acceleration, road traffic annoyance studies etc). Before applying a simulated vehicle in psychoacoustic noise annoyance studies, one should perform a subjective validation of this tool. The present paper presents the subjective validation of simulation method created by the author. Real pass-by recordings were used to calculate the one-third octave power spectra of a vehicle. Based on these spectra, the simulated pass-bys were created. The simulation was validated in terms of annoyance level and perception of velocity by comparing (in 
psychoacoustic experiment) the original pass-bys with their simulated replicas. Two types of engines - otto and diesel - were tested, for velocities ranging from $30-110 \mathrm{~km} / \mathrm{h}$. For velocities above $50 \mathrm{~km} / \mathrm{h}$, the annoyance of simulated pass-bys is very close to the original signal. For lower velocities, simulated pass-bys result in lower annoyance ratings. The solution to the problem would be a reconstruction of the tonal components from the exhaust system.

\section{9:00}

3aPPb4. Experimentation in physics-based sound modeling. Davide Rocchesso (IUAV University of Venice, Department of Art and Design, Dorsoduro 2206, 30123 Venezia, Italy, roc@iuav.it)

Since 2001, as part of the Sounding Object and CLOSED projects, we have been developing physics-based sound models for everyday events and processes. The procedure of describing a sound-producing phenomenon in physical terms, building a model, and accessing its parameters, easily gives the illusion of controlling sound production in the most effective and convenient way. For example, it is trivial to connect a force sensor to the rubbing pressure variable of a friction model. However, one soon realizes that it is often difficult to predict changes in perceived sound in response to physical changes, and that robust models are difficult to achieve. These issues can partially be addressed with experimental research in perception, psychophysics, and phenomenology. Although this approach is inherently time consuming, it will eventually lead to sound models whose parametric layout is more suitable to human manipulation and control. Conversely, in the practice of designers we find that problems are tackled by rapidly producing a multiplicity of attempts, or sketches, and by critically comparing them. In our practice of sound modelers, there are several examples where both approaches, the one based on experimental psychology and the one based on design, proved to be compelling and useful.

\section{9:20}

3aPPb5. Size from sound: Size estimates of balls dropped from various height. Massimo Grassi (Dipartimento di Psicologia Generale, via Venezia 8, 35131 Padova, Italy, massimo.grassi@unipd.it)

Three experiments investigated the perception of the size of a ball (i.e., a non sounding object) from the sound it produces when dropped upon a plate (i.e., a sounding object) from various heights. Experiment one: listeners estimated metrically the size of seven balls $(\varnothing 1,1.5,2,2.5,3,4,5 \mathrm{~cm})$ when dropped upon a baked clay plate from $6 \mathrm{~cm}$ of height. Experiment two: a new group of listeners estimate the size of the balls, however, balls could be dropped from either $6 \mathrm{~cm}$ or $12 \mathrm{~cm}$ of height. In the last experiment, balls could be dropped from either 3,6 or $12 \mathrm{~cm}$ of height. In all experiments listeners received no foregoing information about the sound source event, such as the material of the balls, the material of the plate, etc. Overall, listeners' estimations matched veridically the actual size of the balls. However, in the second and third experiment, listeners' estimations were dependent on the height: balls were judged slightly larger when dropped from the highest heights.

\section{Contributed Paper}

9:40

3aPPb6. The texture of natural sounds. Tjeerd C.
Andringa (University of Groningen, Dept. Artificial Intelligence, P.O. Box 407, 9700 AK Groningen, Netherlands, t.andringa@ai.rug.nl)

The texture, a spectro-temporal pattern, of many sound sources is a robust and characteristic perceptual property that listeners use for sound source recognition. The robustness of the texture ensures that we can recognize sound sources like a helicopter, flowing water, or a surf breaking on pebbles in a wide variety of acoustic environments. This robustness suggests that textures can be used for automatic source identification or environment classification. We introduce a method to determine the presence of sound textures associated with, for example, flat noise, pulsed noises (helicopter), sweep based textures (running water), and tonal noises (babble). The cumulative probability density of time-frequency fluctuations are matched with prototypical cumulative probability density functions (cpdf) with a running variant of the Kolmogorov-Smirnov test. Textures with a similar distribution as the target distribution, contribute approximately equally to all values of the cpdf. The flatness of this distribution is used as a distance measure. When the pdf's of the target textures do not overlap strongly, the method can determine the texture of time-frequency-regions as small as $100 \mathrm{~ms}$ by 6 semi-tones. This method can therefore also be used to determine the texture of the background.

\section{Invited Papers}

3aPPb7. 'Behavioral Relevance' as a guiding principle in investigating the perception of sound source properties. John Neuhoff (The College of Wooster, 930 College Mall, Wooster, OH 44691, USA, jneuhoff@wooster.edu)

Perceiving a sounding object is the result of a complex interaction between physical, physiological, and cognitive factors. However, early in the history of modern psychacoustics, the difficulty in generating complex, controlled, dynamic stimuli limited many investigations of auditory perception to monaural presentation of static sine wave tones or bursts of noise. Thus, very influential models of auditory processing were developed using sounds that are not frequently encountered in a natural listening environment. Although this approach was useful in developing models of the function of the peripheral auditory system, it was less so in developing models of auditory cognition and behavior. If one accepts the position that our perceptual abilities have evolved specifically to help us survive and deal with the stimuli that occur in a natural environment, perhaps it is not surprising that there are differences in processing naturally occurring stimuli and those that are more artificial. Recent paradigmatic shifts spurred by technical innovations have spawned more ecologically valid investigations of complex auditory perception and cognition that include the perception of sound source properties. The future for this approach is promising if "behavioral relevance" can serve a guiding principle. 
10:40

3aPPb8. Classification of everyday sounds: Influence of the degree of sound source identification. Olivier Houix (IRCAM UMR CNRS 9912, Equipe Perception et Design Sonores, 1, place Igor Stravinsky, 75004 Paris, France, olivier.houix @ircam.fr), Guillaume Lemaitre (IRCAM - UMR CNRS 9912, Equipe Perception et Design Sonores, 1, place Igor Stravinsky, 75004 Paris, France, lemaitre@ircam.fr), Nicolas Misdariis (IRCAM - UMR CNRS 9912, Equipe Perception et Design Sonores, 1, place Igor Stravinsky, 75004 Paris, France, misdarii@ircam.fr), Patrick Susini (IRCAM - UMR CNRS 9912, Equipe Perception et Design Sonores, 1, place Igor Stravinsky, 75004 Paris, France, susini@ircam.fr)

Based on a review of everyday sound classification studies, we define three types of similarity that might underlie the formation of categories: acoustical similarity, related to the perception of the sound signal properties; causal similarity, related to the perception of the cause of the sound; semantic similarity, related to the meaning of the sounds. This study focuses on the influence of the degree of sound source identification on the type of similarity used during a free sorting task. Our hypothesis is that well-identified sounds will lead to categories based on causal or semantic similarities, while non-identified sounds will lead to categories based on acoustical similarity. First we measure the causal uncertainty of each sound of a set of kitchen sounds. We ask to participants to describe the cause of the sounds in order to measure the degree of identification for each sound. The results of a free sorting task on this sound corpus indicate that participants groups together sounds according to our assumptions. The classification of the well-identified sounds shows a clear distinction between classes of sound events (gas, liquid, solids, motor) confirming a taxonomy proposed in literature. [Work supported by the CLOSED project of the NEST program]

\section{Contributed Paper}

\section{1:00}

3aPPb9. Verbal imitations of sound events enable recognition of the imitated sound events. Karine Aura (Université de Toulouse II Le Mirail, Laboratoire Octogone-Lordat URI 41 56, IFR96, Pavillon de la Recherche, 5 allée Antonio Machado, 31058 Toulouse, France, aura@univ -tlse2.fr), Guillaume Lemaitre (IRCAM - UMR CNRS 9912, Equipe Perception et Design Sonores, 1, place Igor Stravinsky, 75004 Paris, France, lemaitre@ircam.fr), Patrick Susini (IRCAM - UMR CNRS 9912, Equipe Perception et Design Sonores, 1, place Igor Stravinsky, 75004 Paris, France, susini@ircam.fr)

We report a study investigating how verbal imitations of sound events might convey information enabling the recognition of the imitated sounds. We sampled twenty-eight sounds across a taxonomy of kitchen sounds, obtained from a previous experiment focused on the perceived properties of the causes of the sounds. First, we recorded speakers while imitating the sounds. They were specifically required not to use words. Then we selected the imitations of six speakers (three men, three women) imitating twelve sounds. Finally a group of twenty listeners performed a sorting task (they had to group together imitations thought to imitate the same events), and were asked to describe how they built each category. The categories of imitations, obtained from a hierarchical tree representation, fit very well with the categories of the original sound events. This suggests that speakers have succeeded in capturing the information sufficient to enable listeners to recognize the sound events. This is further confirmed by the analyses of the verbalizations. Phonetic analyses show that imitations grouped together share some phonetic similarities, yet the main similarities seem rather to be based on prosodic indexes (duration, intonation, rhythmic patterns). [This work is founded by the FP6 NEST Pathfinder European project CLOSED]

\section{Invited Papers}

\section{1:20}

3aPPb10. Auditory Perception of Silent Object Properties. Lawrence D. Rosenblum (University of California, Riverside, Department of Psychology, 900 University Ave., Riverside, CA 92521, USA, rosenblu@citrus.ucr.edu)

While much of what we consciously hear is based on sound sources, we also hear properties of objects that are themselves silent, but act to reflect and obstruct sound. A research program has been initiated to examine the degree to which listeners can judge properties of silent, sound-structuring objects. Results of this research have revealed impressive sensitivity to many of these properties. Thus far, this work has shown that (sighted) listeners have some ability to: a) determine the location, dimensions, and general shape of soundobstructing panels; and b) identify various rooms based on how rooms structure emitted sounds; and c) determine their location in a room based on how the room structures sound at different positions. These results indicate that listeners are sensitive to ambient, as well as emitted acoustic properties, and call for a modified conception of human audition that takes into account these sensitivities.

\section{1:40}

3aPPb11. Evaluation and modification of listener decision strategy in human gait perception. Richard E. Pastore (Binghamton University (SUNY), Department of Psychology, Binghamton, NY 13902-6000, USA, pastore@binghamton.edu), Jesse D. Flint (Binghamton University (SUNY), Department of Psychology, Binghamton, NY 13902-6000, USA, jdflint@ binghamton.edu), Jeremy R. Gaston (Binghamton University (SUNY), Department of Psychology, Binghamton, NY 13902-6000, USA, jgaston1@ binghamton.edu)

Beyond obvious differences, there are many similarities in the challenges faced in studies of speech and natural (nonspeech) sounds. The source events are dynamic, complex and highly variable. Source properties map only statistically in the sounds produced, resulting in many acoustic properties that are only sometimes accurate indicators of specific source properties. Finally, beyond the nonlinear mapping of acoustic to perceptual properties, listeners vary in decision weights assigned to the perceived attributes of the sounds. Beginning with this complex conceptualization of source-sound-perception relationships, our research on the perception of human gait from walking sounds investigated the perception of walker posture. The current research extends this project on human gait perception, evaluating both the decision strategy of listener individual listeners and alternative approaches to improving that decision strategy. Implications for studying of both natural nonspeech and speech will be briefly discussed. 


\title{
Contributed Papers
}

12:00

3aPPb12. Source Perception of Everyday and Self Produced Sounds: Factors in the Evolution of Human Auditory Cognitive Capability. James Ballas (Naval Research Laboratory, 4555 Overlook AVE SW, Washington, DC 20375, USA, james.ballas@nrl.navy.mil)

The everyday world was making meaningful sound well before language developed. Self-produced sound exhibits a tight binding between source event and interpretation. These assertions naturally prompt thoughts about the role of these types of sound in the evolution of human auditory capability, especially language. The recent resurgence in theorizing about the origin of language has included other human capabilities as potential generative factors (e.g., gestures), but there has been little discussion about the possible role of capability to process everyday and self-produced sound. This is surprizing given that recent neurocognitive research has supported common processing for speech and meaningful sound. Key attributes of language processing are the ability to interpret not just signs but symbols, and the ability to employ generative grammar processing to produce and interpret symbol strings. Association of sound events with causes and sequences, auditory representation of meaning, persistence of meaning under event and sequence variation, and persistence of event interpretations beyond what is currently present (temporal and spatial abstraction) are all fundamental requirements for these language attributes, and it is asserted that these capabilities might have developed to accurately perceive everyday and selfproduced sound.
12:20

3aPPb13. Detection of missing modal frequencies. Christophe $\mathrm{N}$. Stoelinga (University of Wisconsin-Madison, Department of Communicative Disorders, 1975 Willow drive, Madison, WI 53706, USA, stoelinga@wisc.edu), Robert A. Lutfi (University of Wisconsin-Madison, Department of Communicative Disorders, 1975 Willow drive, Madison, WI 53706, USA, ralutfi@wisc.edu)

The amount of information about a source that can be recovered from sound is naturally limited by the ear's ability to resolve individual modal frequencies unique to that source. To measure these limits, listeners were asked to detect, in a standard two-interval, forced-choice task with feedback, which of two sounds on each trial contained a missing partial. The frequencies of the partials corresponded to the ideal modes of a simply-supported, rectangular plate. Plate surface areas and height-wide ratios were chosen to produce the same bandwidths $(125-1125,250-2250$ or $500-4500 \mathrm{~Hz})$ for different numbers of partials $(11,16$ or 24). Overall level of the sounds was roved to discourage detection based on simple level differences. Detection of five highly-practiced listeners was largely independent of the frequency of the lowest partial, being best for partials 1-3, 1-4 or 1-6 for 11,16 or 24 partials in total, respectively. Mutual masking among the higher-number partials is given as the likely cause. The results are discussed in terms of their implications for the identification of rudimentary source attributes from sound. [Work supported by NIDCD grant 5 R01 DC006875].

WEDNESDAY MORNING, 2 JULY 2008

ROOM 241, 9:00 A.M. TO 12:40 P.M.

Session 3aPPc

\section{Psychological and Physiological Acoustics, Architectural Acoustics, Noise, and Signal Processing in Acoustics: Jens Blauert and His Contributions}

\author{
Ervin R. Hafter, Cochair \\ University of California, Department of Psychology, 3210 Tolman Hall, Berkeley, CA, CA 94720-1650, USA
}

Armin Kohlrausch, Cochair

Philips Research Europe, Digital Signal Processing (MS WO02), High Tech Campus 36, Eindhoven, 5656 AE, Netherlands

\section{Invited Papers}

3aPPc1. A means for comparing normal and impaired listeners in spatial hearing. Ervin R. Hafter (University of California, Department of Psychology, 3210 Tolman Hall, Berkeley, CA, CA 94720-1650, USA, hafter@ berkeley.edu)

\footnotetext{
In our computer controlled Simulated Open Field Environment (SOFE), a computer generates simulated sources and echoes in an anechoic chamber. The SOFE has evolved over many years through ideas and hard work from a superb group of graduate students and post docs including Francoise Briolle, Tom Buell, Poppy Crum, Eric Jensen, Kourosh Saberi, Chris Stecker, Bernhard Seeber, and Miriam Valenzuela, as well as advice from such luminaries as Georg von Bekesy, Arnold Leiman and, of course, Jens Blauert. The ready use of Head Related Transfer Functions (HRTFs) with headphones raises the question, "Why the SOFE?" The answer is that each subject enters the environment with his or her natural HRTF and hearing apparatus intact, thus allowing comparisons between patients with cochlear implants or hearing aids, as well as listeners with normal hearing. Since its inception, this environment has been used to examine precedence, the role of relative level in echo suppression, multiple factors in auditory motion perception, and ventriloquism. This presentation will review some of the more interesting results.
} 
3aPPc2. The salience of interaural temporal disparities (ITDs) conveyed by high-frequency signals is determined by the temporal features of their ongoing envelopes. Constantine Trahiotis (University of Connecticut Health Center, Departments of Neuroscience and Surgery (Otolaryngology), 263 Farmington Avenue, Farmington, CT 06030-3401, USA, tino@neuron .uchc.edu), Leslie R. Bernstein (University of Connecticut Health Center, Departments of Neuroscience and Surgery (Otolaryngology), 263 Farmington Avenue, Farmington, CT 06030-3401, USA, les@ neuron.uchc.edu)

Many studies of binaural hearing reveal that envelopes of complex, high-frequency stimuli convey useful interaural time differences (ITDs). Envelope-based ITDs have, typically, been found to be less "potent" than those conveyed by low-frequency waveforms. When considering such findings, Blauert, in his classic textbook, noted that monaural, peripheral processing would result in 1) low-frequency inputs to the binaural processor that are essentially half-wave rectified versions of the input waveforms and 2) high-frequency inputs that are essentially full-wave rectified versions of only the envelopes of the input waveforms. Taking this into account, Blauert, echoing Colburn and Esquissaud (1976), surmised that low-frequency signals would ultimately convey "more distinct time cues" having greater "transient features" than would high-frequency signals and, therefore, be expected to provide more precise ITD information. By using high-frequency "transposed" stimuli having envelopes constructed to provide "more distinct time cues" we have shown in a variety of experimental contexts that the potency of envelope-based ITDs, can rival that measured with low-frequency stimuli. Our most recent experiments use "raised sine stimuli" in order to vary directly the temporal features of the envelope waveform including its "sharpness," "off-time," and interaural cross-correlation function. The new data will be discussed in terms of Blauert's insight.

\section{9:40}

3aPPc3. Cognitive influences on spatial hearing. Rachel Keen (University of Virginia, Department of Psychology, P. O. Box 400400, Charlottesville, VA 22904-4400, USA, rachelkeen@ virginia.edu)

In reverberant rooms a listener perceives a sound coming from its source but simultaneously suppresses reflected sound or echoes. This phenomenon is known as the precedence effect because the preceding (original) sound is given heavier weight than its subsequent reflections. Our research has explored this perceptual process and found that listeners are sensitive to the acoustic information contained in the reflected sound even though it is "suppressed". Specifically, below echo-threshold information relevant to room acoustics (e.g., delay between lead and lag sounds, filtering of the echo's spectrum, presence or absence of an echo at a particular location) is processed by the listener and influences perception. Listeners' expectations about ongoing input can raise or lower echo threshold. Recent ERP data on listeners' responses to click pairs near echo threshold found that when listeners perceived lead and lag sounds as separate sources, a negativity between 100 and $250 \mathrm{~ms}$ was elicited, termed object-related negativity (ORN). When the same stimulus was heard as a single fused sound, no ORN was elicited. These findings suggest that higher-level cognitive processes exert top-down influences over early neurosensory processing.

\section{0:00}

3aPPc4. Modeling the Precedence Effect -- how we arrived where we are today. Jonas Braasch (Rensselaer Polytechnic Institute, Greene Bldg., 110 8th St., Troy, NY 12180, USA, braasj@rpi.edu)

Throughout his career, Jens Blauert was interested in further exploring the precedence effect, an auditory phenomenon that enables us to localize sounds in rooms and other enclosures. A series of models that were developed at Bochum's Institute of Communication Acoustics under his leadership have changed the way we think about the precedence effect. While this effect as a psychoacoustic phenomenon is known for its complexity -- for which Jens Blauert's talent to define precise terminology and listen critically came in handy -- the modeling aspects are equally challenging due to the non-linear nature of the underlying auditory processes. Blauert and Cobben's binaural model from 1978 was already able to simulate observations related to the precedence effect. A few years later, Blauert's doctoral student Werner Lindemann introduced a binaural model with specific stages to model the precedence effect. Since then, almost all approaches to simulate the precedence effect are based on inhibitory elements. In this talk, the evolution of this class of models over several student generations at Bochum will be described, and the fruitful scientific exchange with other institutions such as Steven Colburn's laboratory at Boston University will be highlighted as well.

\section{0:20}

3aPPc5. A History of Binaural Interaction Modeling - Walks with Jens Blauert. H Steven Colburn (44 Cummington St., Boston, MA 02215, USA, colburn@bu.edu), Suzanne Carr (44 Cummington St., Boston, MA 02215, USA, ruiwan@bu.edu), Rui Wan (44 Cummington St., Boston, MA 02215, USA, suzy@bu.edu)

Jens Blauert has been cut an impressively wide path through diverse areas of binaural and spatial hearing. He has consistently integrated viewpoints based on empirical results, intuitive interpretations, and mathematical and computational models of binaural interaction and spatial hearing. This talk focuses on the development of ideas about binaural interaction as represented in quantitative models and the strong influence that Blauert exerted on these models. These influences start with simple models of the extraction and use of interaural time and level differences, include mechanisms that incorporate complex interactions of these differences, and lead to models that are appropriate for the interpretation of complex acoustic scenes. Finally, some current modeling activities will be outlined and related to these historical developments. [Work supported by the US National Institutues of Health DC00100.] 
3aPPc6. Localization-based segregation of acoustic sources: Advantages and limitations. Pierre Divenyi (VA Northern Calif. Health Care Syst. and East Bay Inst. for Research and Education, Bldg. R4, 150 Muir Rd., Martinez, CA 94553, USA, pdivenyi@ebire .org)

It has been suggested [Divenyi, P., \& Oliver, S.K., (1989), JASA 85, 2042-2052] that segregation of simultaneous pairs of sounds in the horizontal plane requires both resolution of the azimuthal separation between the sources, and correct assignment of each signal to the correct source. In fact, contrary to our a priori expectations, localization did not emerge as the dominant dimension underlying segregation of source pairs: its importance often fell behind that of segregation based on differences of spectral or temporal envelope structure, except for some listeners. Nevertheless, the possibility remains that these limitations can be attributed to localization dominance by one of the sources or both. The dual requirement of localization and signal-to-source assignment, in addition to reverberation, may represent a limiting factor for the role of localization in the cocktail-party effect.

\section{Contributed Paper}

\section{$11: 20$}

3aPPc7. From binaural hearing to auralization: A triumph of Blauert's 40 years in room acoustics. Ning Xiang (Rensselaer Polytechnic Institute, Greene Building, School of Architecture, 110 8th Street, Troy, NY 12180, USA, xiangn@rpi.edu), Wolfgang Hess (Harman/Becker Automotive Systems, EG - Advanced Signal Processing, 76307 Karlsbad, Germany, WHess@harmanbecker.com)

In the late 60's when Jens Blauert's work on sound localization in the median plane was recognized by the psychoacoustics community, he had already started on a long journey from binaural-hearing research to many aspects of what nowadays is called Communication Acoustics. In addition to initiating many new research activities such as binaural auditory modelling, binaural technology, sound-quality, and speech synthesis, his unremitting endeavour in room acoustics has led to innovative developments in the fields of analysis and synthesis of auditory scenes. For example, binaural roomacoustics modelling, real-time dynamic room-impulse response processing, and auditory virtual environments (nowadays known as "auralization") were particularly driven by his work and his supervision of over 50 doctoral students. In this regard the first head-tracking and fast-convolution patent application was filed in the early 70's. Recently auditory models have been applied in auditory spatial evaluations of rooms and cars. Not only his excellent analytical skills and theoretical knowledge, but also his vision for transfer to practical applications in room acoustics and human-auditory spatial perception led to an outstanding life-work. This paper will highlight just a few of Jens Blauert's major contributions to room-acoustics.

\section{Invited Papers}

\section{1:40}

3aPPc8. Binaural technology for e.g. rendering auditory virtual environments. Dorte Hammershøi (Acoustics, Aalborg University, Fredrik Bajers Vej 7 B5, 9220 Aalborg Ø, Denmark, dh@es.aau.dk)

Jens Blauert's research up through the late 1960s and later, pioneered the field of binaural technology and auditory virtual environments. He mastered the measurement of head-related transfer functions (HRTFs) before the term was introduced, and his methods were standard for decades. While most acknowledge his efforts in understanding the binaural hearing and the significance of interaural differences, appreciation is also in place for his evaluation of localization with identical ear input signals. The relations between the hearing's "directional" bands and the transfer functions' "boosted" bands, helped mediate the understanding that if the transfer functions could be mastered, then important dimensions of the auditory percept could also be controlled. He early understood the potential of using the HRTFs and numerical sound transmission analysis programs for rendering auditory virtual environments. Jens Blauert participated in many European cooperation projects exploring this field (and others), among other the SCATIS project addressing the auditory-tactile dimensions in the absence of visual information.

\section{2:00}

3aPPc9. How can we model a quality event? Some considerations on describing quality judgment and prediction processes. Sebastian Möller (Deutsche Telekom Laboratories, Berlin Institute of Technology, Ernst-Reuter-Platz 7, 10587 Berlin, Germany, sebastian.moeller@telekom.de)

Jens Blauert has introduced a systemic view on a listener in an auditory experiment. This view helps to separate sound events from auditory events and from their descriptions, and to identify and describe the processes involved in such experiments. The notion has later been extended to listeners in a quality judgment situation by Jekosch and Raake, leading to the notion of a "quality event". On the one hand, knowledge of the involved processes is necessary to design appropriate measurement processes for, e.g., sound quality, transmission quality, auditory-scene quality, or product-sound quality. On the other hand, such knowledge enables us to define algorithms which estimate quality - or sub-aspects of it - in the system design process. The talk will mainly follow the second line and will identify components which are necessary for an algorithmic description of the processes involved in the formation of a quality event. Taking the example of telecommunication services, it will be shown which components of quality prediction models are already available, and which others are still out-of-reach and require further study.

3aPPc10. Semio-acoustics: a domain of communication acoustics. Ute Jekosch (Chair of Communication Acoustics, TU Dresden, Helmholtzstr. 10, 01069 Dresden, Germany, ute.jekosch@tu-dresden.de)

For user-oriented design of modern information and communication systems we investiate the perception of acoustic signals as sign carriers: How do listeners process acoustic/auditory events when they treat them as information carriers and which meaning do they associate with them? Sign carriers (in our case the acoustic signals) transport information across space and time. When being perceived, they are decoded, analyzed and interpreted with regard to their cultural meaning and their relevance for the actual living and commu- 
nication situation. These processes are universal to a certain degree. It is the aim of our research in Dresden to investigate the fundamental structures and organisational principles underlying this assignment of meaning to acoustic and their accompanying auditory events. In this talk, some semio-acoustic paradigms will be introduced. As a Ph.D. student of Jens Blauerts' and as a post doc in his team, the semio-acoustic approach has developed as a logical continuation of his way of thinking, of his research approaches, and of his scientific goals, based on and fed by his basic statement: Communication acoustics is not conceivable without audition, and only if we are able to understand auditory events, and even cognitive processes, we are able to make use of acoustic communication and information technology in a user-friendly way.

WEDNESDAY MORNING, 2 JULY 2008

ROOM 252B, 8:00 TO 10:00 A.M.

\title{
Session 3aSAa
}

\section{Structural Acoustics and Vibration, Computational Acoustics, and EURONOISE: Efficient Boundary Element Methods I}

\author{
Ramani Duraiswami, Cochair \\ University of Maryland Institute for Advanced Computer Studies, 115 A.V. Williams Bldg, College Park, MD 20742, USA \\ Lothar Gaul, Cochair \\ Institute of Applied and Experimental Mechanics, University of Stuttgart, Pfaffenwaldring 9, Stuttgart, 70550, Germany
}

Invited Papers

8:00

3aSAa1. Vibroacoustic Simulation of Partly Immersed Bodies by a Coupled Fast BE-FE Approach. Dominik Brunner (Institute of Applied and Experimental Mechanics, University of Stuttgart, Pfaffenwaldring 9, 70550 Stuttgart, Germany, brunner@iam.uni-stuttgart.de), Michael Junge (Institute of Applied and Experimental Mechanics, University of Stuttgart, Pfaffenwaldring 9, 70550 Stuttgart, Germany, junge@iam.uni-stuttgart.de), Christian Cabos (Germanischer Lloyd, Vorsetzen 35, 20459 Hamburg, Germany, christian.cabos@gl-group.com), Lothar Gaul (Institute of Applied and Experimental Mechanics, University of Stuttgart, Pfaffenwaldring 9, 70550 Stuttgart, Germany, gaul@iam.uni-stuttgart.de)

Simulation of vibroacoustic behavior of submerged bodies necessitates dealing with fluid-structure coupled problems. In case of a high density of the fluid, the feedback of the acoustic pressure onto the structure cannot be neglected and a fully coupled system must be investigated. In this work, the finite element method (FEM) is used for the structural part. The commercial finite element package ANSYS is applied for setting up the mass and stiffness matrices. The boundary element method (BEM) is well suited for simulating the sound propagation in the unbounded exterior acoustic fluid domain. Here, the fast multipole method (FMM) is applied to overcome the known bottleneck of classical BE-methods. In the case of partly immersed bodies, Dirichlet boundary conditions on the fluid surface additionally have to be incorporated. This is done by applying a halfspace formulation. The extension of the FMM to this scenario is discussed. Iterative preconditioned solvers are employed and the numerical efficiency is discussed. The applicability of the coupling scheme is demonstrated using a realistic model problem.

$8: 20$

3aSAa2. A half-space BEM for the simulation of sound propagation above an impedance plane. Haike Brick (TFH Berlin University of Applied Sciences, FB II, Mathematics - Physics - Chemistry, Luxemburger Str. 10, 13353 Berlin, Germany, brick@tfh-berlin.de), Martin Ochmann (Technische Fachhochschule Berlin, Univ. of Applied Sciences, Luxemburger Str. 10, 13353 Berlin, Germany, ochmann@tfh-berlin.de)

The Boundary-Element-Method is a powerful tool for the simulation of sound radiation and scattering. Classically, it was developed for the free 3D-space, but it can be modified easily for half-space solutions as long as the half-space is delimited by a perfectly rigid or soft plane. In this case, the Green's function, the core of the BEM, can be derived from a simple image source ansatz, which however cannot be used for a more general impedance boundary condition. The alternative, an additional discretisation of a finite but large part of the plane leads to an enormous increase of the size of the set of equations. In this presentation, an appropriate Green's function will be introduced, which is able to describe the sound propagation above an impedance plane and is suitable for an implementation into a BEM code. It bases on the superposition of sound sources with complex source points. The numerical evaluation of this Green's function will be presented along with several test cases including sound radiation from burning flames above ground and tire-road noise. The computational costs of the developed "Complex-Source-Point-BEM" (CBEM) in comparison with a classical BEM together with a discretisation of the impedance plane will be discussed. 


\section{Contributed Papers}

\section{8:40}

3aSAa3. Simulation of noise barrier insertion loss using the boundary element method. Holger Waubke (Austrian Academy of Sciences / Acoustics Research Institute, Wohllebengasse 12-14, 1040 Vienna, Austria, holger.waubke@oeaw.ac.at), Wolfgang Kreuzer (Austrian Academy of Sciences / Acoustics Research Institute, Wohllebengasse 12-14, 1040 Vienna, Austria, wolfgang.kreuzer@oeaw.ac.at), Zhensheng Chen (Austrian Academy of Sciences / Acoustics Research Institute, Wohllebengasse 12-14, 1040 Vienna, Austria, zhensheng.chen@oeaw.ac.at)

Within a project of the European Commission "Calm Tracks \& Routes" the performances of different styles of noise barriers were investigated. Additionally new methods for increasing the absorption were investigated. A central theme was to investigate the mitigation of curved walls with high absorption coefficients, because studies made before the project gave the hint that noise barriers curved away from the source should give satisfying insertion loss. Due to the curvature the view in the landscape is hindered to a lesser degree compared to straight noise barriers. The investigation of the noise barriers was done by boundary element methods. An absorptive model for grassland was added and implemented into Green's functions. Due to the existence of smeared sources the application of the fast multipole method was not possible and the simulations were limited to the two-dimensional case. It became obvious that the distance of the source is important for the mitigation of curved noise barriers with respect to straight ones. Therefore different wall types and source positions were investigated. The results of the numerical simulation were compared to measurements in situ and in a large anechoic chamber.

\section{9:00}

3aSAa4. A GWBEM method for high frequency acoustic scattering Emmanuel Perrey-Debain (Université de Technologie de Compiègne, Lab. Roberval UMR 6253, Dept. Acoustique, BP 60319, 60203 Compiègne, France, emmanuel.perrey-debain@utc.fr), Hadrien Bériot (Université de Technologie de Compiègne, Lab. Roberval UMR 6253, Dept. Acoustique, BP 60319, 60203 Compiègne, France, beriotha@ gmail.com), Mabrouk Ben Tahar (Université de Technologie de Compiègne, Lab. Roberval UMR 6253, Dept. Acoustique, BP 60319, 60203 Compiègne, France, mabrouk.bentahar@utc.fr), Catherine Vayssade (Université de Technologie de Compiègne, Lab. Roberval UMR 6253, Dept. Acoustique, BP 60319, 60203 Compiègne, France, catherine.vayssade@utc.fr)

This paper considers the problem of scattering of a time-harmonic acoustic incident wave by a hard obstacle. The numerical solution to this problem is found using a Galerkin Wave Boundary Element Method (GWBEM) whereby the functional space is built as the product of conventional low order piecewise polynomials with a set of plane waves propagating in various directions. In this work we present strategies for finding the appropriate plane wave basis locally on each boundary element in order to deal efficiently with very irregularly meshed structures exhibiting both large smooth scattering surfaces as well as corners and small geometrical features. Numerical results clearly demonstrate that these improvements allow the handling of scatterers with complicated geometries while maintaining a low discretization level of 2.5 to 3 degrees of freedom per full wavelength.

$$
\text { 9:20 }
$$

3aSAa5. Fast multipole accelerated boundary element method (FMBEM) for solution of 3D scattering problems. Nail A. Gumerov (University of Maryland Institute for Advanced Computer Studies, 115 A.V. Williams Bldg, College Park, MD 20742, USA, gumerov @ umiacs.umd.edu), Ramani Duraiswami (University of Maryland Institute for Advanced Computer Studies, 115 A.V. Williams Bldg, College Park, MD 20742, USA, ramani@umiacs.umd.edu)

Wideband FMBEM codes are challenging to implement since there are problems at both very low and high frequencies. Substantially different schemes for function representation and translation are efficient for low and high frequency ranges. We present a method which is suitable for solution both high and low frequency problems since it implements a switch between different representations and uses fast translation methods appropriate to each representation. For a high frequency problem the switch between representations may occur at some intermediate levels of hierarchical space subdivision of the FMM. We also present an FMM-based preconditioner used in the flexible GMRES iterative solver for scattering problems and discuss example problems computed in range $0.001<\mathrm{kD}<200$, where $\mathrm{k}$ is the wave number and $\mathrm{D}$ is the size of the computational domain. The theoretical and experimental computational complexity of our algorithm is approximately $\mathrm{O}\left((\mathrm{kD})^{3}\right)$ at large $\mathrm{kD}$ with relatively small asymptotic constant and the algorithm allows to solve problems with up to million nodes used for surface discretization in the range tested on a conventional personal computer.

\section{9:40}

3aSAa6. An efficient distributed BEM solver for acoustic and vibroacoustic analyses on a standard PC network. Paolo Di Francescantonio (STS, Via Dalmazia 30, 21100 Varese, Italy, pdifra@stsweb.it)

An efficient BEM approach that permit to solve large acoustic and vibroacoustic problems distributing analyses on a standard PC network is presented. The high efficiency is based on a mix of elements including: 1) A proprietary and high efficient communication and synchronization library (not MPI) 2) A Multi Domain Approach for reducing memory requirements and solution time 3) Efficient iterative solvers The proprietary communication / synchronization library permits to take full advantage of recent multi core PC, enabling to set up clusters with a large number of computing units with a reduced number of PC. A "distributed memory" approach permit to have available the sum of all the PCs RAM, ensuring the possibility to solve large and huge problems that cannot be faced with standard approaches. The Multi Domain approach permit to further reduce the memory requirements, while preserving all the required flexibility in terms of boundary condition and coupling with structural modes. Examples will be showed of large an huge analyses in different application fields including purely acoustics, and fully coupled structural-acoustic analyses. 


\title{
Session 3aSAb
}

\section{Structural Acoustics and Vibration, Computational Acoustics, and EURONOISE: Fluid-Structure Interaction I}

\author{
Noureddine Atalla, Cochair \\ Univ. de Sherbrooke, Mechanical Engineering Depart., 2500 Boulevard de l'Université, Sherbrooke, QC J1K 2RI, Canada
}

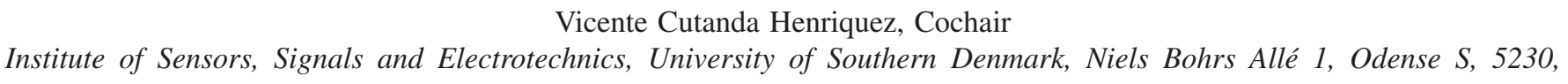
Denmark

Stefan Schneider, Cochair

Laboratoire de Mécanique et d'Acoustique, UPR 7051 CNRS, 31,Chemin Joseph-Aiguier, Marseille, 13402, France

\section{Invited Papers}

\section{0:40}

3aSAb1. Practical modeling of acoustic losses in air due to heat conduction and viscosity. René Christensen (Oticon A/S, Kongebakken 9, 2765 Smoerum, Denmark, rch@oticon.dk), Peter Juhl (Institute of Sensors, Signals and Electrotechnics, University of Southern Denmark, Niels Bohrs Allé 1, 5230 Odense S, Denmark, pmjuhl@ sense.sdu.dk), Vicente Cutanda Henriquez (Institute of Sensors, Signals and Electrotechnics, University of Southern Denmark, Niels Bohrs Allé 1, 5230 Odense S, Denmark, vch@sense.sdu .dk)

Accurate acoustics models of small devices with cavities and narrow slits and ducts should include the so-called boundary layer attenuation caused by thermal conduction and viscosity. The purpose of this paper is to present and compare different methods for including these loss mechanisms in analytical and numerical models. A simple circular geometry with a narrow tube has been used as a reference and is investigated both through measurements and the different models. The simulation methods compared are: i) traditional analytical approaches such as lumped parameter modelling and transmission line modelling, ii) numerical methods implemented into commercial packages, such as the low reduced frequency models as proposed by W. M. Beltman and implemented in ACTRAN and the linearized Navier-Stokes equations used in COMSOL Multiphysics, and iii) an implementation specifically made for this purpose using BEM and the full linearized model by M. Bruneau.

\section{1:00}

3aSAb2. An overview of models for viscothermal wave propagation, including fluid structure interaction. Willem M. Beltman (Intel Corporation, $2111 \mathrm{NE}$ 25th Avenue, M/S JF2-86, Hillsboro, OR 97124, USA, willem.m.beltman@intel.com), Ysbrand H. Wijnant (University of Twente, Dept. Mechanical Engineering, P.O. Box 217, 7500 AE Enschede, Netherlands, Y.H.Wijnant@ctw.utwente.nl), Marten J. Nijhof (University of Twente, Dept. Mechanical Engineering, P.O. Box 217, 7500 AE Enschede, Netherlands, m.j.j.nijhof@ctw.utwente.nl)

In acoustics, the standard wave propagation models neglect the effects of viscosity and thermal conductivity. When waves propagate in narrow tubes or thin layers these simplifications might not be accurate. This paper presents an overview of models that take the effects of inertia, viscosity, thermal conductivity and compressibility into account. Based on the use of dimensionless parameters, three classes of models are outlined. The most important dimensionless parameter is the shear wave number, an unsteady Reynolds number that indicates the ratio between inertial and viscous effects. These viscothermal wave propagation models can be coupled to structural models to capture the fluid structure interaction. Analytical solutions can be found for these coupled analysis cases only for simple geometries and boundary conditions. For more complex geometries, numerical models were developed. Examples of applications of these models are also presented.

\section{1:20}

3aSAb3. A 3 dimensional finite element modeling of "smart foam" and its experimental validation. Pierre M. Leroy (Laboratoire de Mécanique et d'Acoustique - CNRS, 31 chemin Joseph Aiguier, 13402 Marseille, France, leroy@1ma.cnrs-mrs.fr), Noureddine Atalla (Univ. de Sherbrooke, Mechanical Engineering Depart., 2500 Boulevard de 1'Université, Sherbrooke, QC J1K 2R1, Canada, Nouredinne.Atalla@Usherbrooke.ca), Alain Berry (Univ. de Sherbrooke, Mechanical Engineering Depart., 2500 Boulevard de l'Université, Sherbrooke, QC J1K 2R1, Canada, alain.berry@usherbrooke.ca), Philippe Herzog (Laboratoire de Mécanique et d'Acoustique - CNRS, 31 chemin Joseph Aiguier, 13402 Marseille, France, herzog@1ma.cnrs-mrs.fr)

The "smart foam" concept and design originate from the combination of passive dissipation of acoustic foams in the medium and high frequency range and the active absorption ability of piezoelectric actuators (generally PVDF) in the low frequency range. This results in a passive/active absorption control device that can efficiently operate over a broad range of frequencies. In this paper, a 3D finite element model of smart foam and its experimental validation are presented. The finite element model uses quadratic poroelastic elements with a (u,p) formulation, as well as elastic, fluid and piezoelectric elements. The weak integral formulation of the different 
involved domains and their coupling conditions are presented. In particular, an isotropic and a simplified orthotropic model of poroelastic media are presented and compared. The fabrication of a prototype smart foam based on melamine foam with a curved, bonded PVDF film is discussed. Passive absorption and acoustic radiation of this prototype are measured in an impedance tube. The measured data are in good agreement with the numerical results, thereby validating the finite element model. This modeling tool constitutes a powerful platform to simulate and optimize various configurations of smart foams.

\section{1:40}

3aSAb4. Prediction of the sound field in anechoic rooms : comparison of two different approaches. François-Xavier Bécot (Matelys - Acoustique \& Vibrations, 20/24 rue Robert Desnos, 69120 Vaulx-en-Velin, France, francois-xavier.becot @matelys.com), Stefan Schneider (Laboratoire de Mécanique et d'Acoustique, UPR 7051 CNRS, 31,Chemin Joseph-Aiguier, 13402 Marseille, France, schneider@lma.cnrs-mrs.fr), Luc Jaouen (Matelys - Acoustique \& Vibrations, 20/24 rue Robert Desnos, 69120 Vaulx-en-Velin, France, luc.jaouen@matelys.com)

Despite the numerical advances, predicting the sound field in anechoic rooms is still challenging because it requires a fine modeling of the wall surface, acoustic properties and geometry, and the computation of the room sound field. It is proposed here to compare two innovative approaches to predict the performance of anechoic wedges, in particular to quantify the influence of porous frame motion, and to propose a numerically efficient model for the prediction of the sound field in anechoic room sounds at low frequencies. The first approach relies on the theory for modeling the sound propagation in double porosity media first presented by Olny \& Boutin [J. Acoust. Soc. Am. 114 (1), 2003]. It has been shown that this approach could accurately predict the cut-off frequency of anechoic wedges. The interest is that this approach is numerically costless and that complex geometry could be considered. The second approach is based on the combination of FEM and BEM. It allows to predict the sound field in anechoic rooms. Compared to the previous approach, the latter one has the advantage of being able to identify structural effects inside the wedges and to propose an accurate prediction of the sound field in the room.

\section{2:00}

3aSAb5. Improvements on the directional characteristics of a calibration sound source using the Boundary Element Method. Vicente Cutanda Henriquez (Institute of Sensors, Signals and Electrotechnics, University of Southern Denmark, Niels Bohrs Allé 1, 5230 Odense S, Denmark, vch@sense.sdu.dk), Salvador Barrera-Figueroa (Danish Fundamental Metrology, Matematiktorvet 307, 2800 Kgs. Lyngby, Denmark, sbf@dfm.dtu.dk), Peter Juhl (Institute of Sensors, Signals and Electrotechnics, University of Southern Denmark, Niels Bohrs Allé 1, 5230 Odense S, Denmark, pmjuhl@sense.sdu.dk)

The project Euromet-792 aims to investigate and improve methods for secondary free-field calibration of microphones. In this framework, the comparison method is being studied at DFM in relation to the more usual substitution method of microphone calibration. The design of the sound source is of particular importance to achieve a sound field that reaches both microphones with the same level and that is sufficiently uniform at the microphone positions, in order to reduce the effect of misalignment. An existing sound source has been modeled using the Boundary Element Method, and the simulations have been used to modify the source and make it suitable for this kind of calibration. It has been found that a central plug, already present in the device, can be re-shaped in such a way that makes the sound field on the microphone positions more uniform, even at rather high frequencies. Measurements have been carried out in order to verify the goodness of this solution.

\section{2:20}

3aSAb6. Analysis of the resonance frequency shift in cylindrical cavities containing a sphere and its prediction based on the Boltzmann-Ehrenfest principle. Arturo Santillan (Universidad Nacional Autonoma de Mexico, Centro de Ciencias Aplicadas y Desarrollo Tecnologico, CCADET-UNAM, Circuito Exterior s/n, Cd. Universitaria, A. P. 70-186, 02510 Mexico, D.F., Mexico, arturo.orozco@ccadet.unam.mx), Vicente Cutanda Henriquez (Institute of Sensors, Signals and Electrotechnics, University of Southern Denmark, Niels Bohrs Allé 1, 5230 Odense S, Denmark, vch@sense.sdu.dk)

It is known that forces generated by high-level acoustic waves can compensate for the weight of small samples, which can be suspended in a fluid. To achieve this, a standing wave is created in a resonant enclosure, which can be open or closed to the external medium. This phenomenon, called Acoustic levitation, has numerous applications in containerless study and processing of materials. Although it is possible to levitate a sample for long periods of time, instabilities can appear under certain conditions. One of the causes of oscillational instabilities is the change of the resonance frequency of the cavity due to the presence of the levitated object. The Boltzmann-Ehrenfest principle is used to find an analytical expression for the resonance frequency shift in a cylindrical cavity produced by a small sphere, with $k r<1$, where $k$ is the wavenumber and $r$ is the radius of the sphere. The validity of this expression has been investigated by means of the Boundary Element Method and experiments. In addition, the effect of the dispersion of the sound field by the sphere and the boundaries of the cavity on the resonance frequency shift has been analyzed using the BEM.

\section{2:40-2:00 Lunch Break}




\section{Invited Paper}

2:00

3aSAb7. A hybrid SEA/image sources approach for the prediction of the insertion loss of enclosures. Franck C. Sgard (IRSST, Service de la recherche, 505 Boulevard de Maisonneuve O, Montréal, QC H3A3C2, Canada, frasga@irsst.qc.ca), Hugues Nelisse (IRSST, Service de la recherche, 505 Boulevard de Maisonneuve O, Montréal, QC H3A3C2, Canada, hugnel@irsst.qc.ca), Noureddine Atalla (Univ. de Sherbrooke, Mechanical Engineering Depart., 2500 Boulevard de l'Université, Sherbrooke, QC J1K 2R1, Canada, Nouredinne.Atalla@Usherbrooke.ca), Celse Kafui Amedin (GAUS, Université de Sherbrooke - 2500 Bd de l'Université, Sherbrooke, QC J1K2R1, Canada, Celse-kafui.amedin@Usherbrooke.ca), Rémy Oddo (GAUS, Université de Sherbrooke - 2500 Bd de l’Université, Sherbrooke, QC J1K2R1, Canada, Remy.Oddo@USherbrooke.ca)

Enclosures are a classical solution to reduce the sound exposure of workers to the noise radiated by machinery. Their acoustic design can be achieved with the help of predictive tools based on simple analytical tools or sophisticated numerical deterministic models. However, there is no simple and fast tool allowing to account for the complexity of the enclosure configuration, capable of better simulating the non-diffuse nature of the field inside the enclosure and covering the typical frequency range $[100 \mathrm{~Hz} ; 5000 \mathrm{~Hz}]$. This paper presents the development of such a tool for the prediction of the acoustic performance of enclosures. It is based on a hybrid model: the statistical energy analysis (SEA) for the sound transmission across the various elements of the enclosure and the method of image sources for the sound field inside the enclosure. The approach is validated by comparing calculation and experimental results carried out in a semi-anechoic room on rectangular and L-shape enclosures for several inner source locations. The effect of an opening is also investigated. The comparisons between the models and the experimental results show a good agreement for most of the tested configurations.

\section{Contributed Papers}

\section{2:20}

3aSAb8. Vibroacoustic interface conditions between prestressed structures and moving fluids. Fabien Treyssède (Nantes Atlantique University - LCPC, Division for Metrology and Instrumentation, Route de Pornic - BP 4129, 44341 Bouguenais, France, fabien.treyssede@lcpc.fr)

Many applications involve coupling between prestressed solids and fluids (possibly flowing). Typical problems might be given by vibroacoustics of fluid-filled pressurized cavities, wave propagation, dynamics and stability of pipes conveying fluids. The goal of this work is to investigate jump conditions that hold for small linear perturbations at any impermeable interfaces, slip or bonded, plane or not, between fluids and structures in the presence of initial flow and prestress. First, the mixed Eulerian-Lagrangian description is briefly recalled. It yields an interesting unification between existing formulations for inviscid fluids (Galbrun's equation) and solids (updated Lagrangian formulation). Based on conservative equations obtained from this description, interface conditions are derived in an elegant manner thanks to the concept of generalized functions in distribution theory. These conditions are written in terms of a curvilinear coordinate system attached to the interface. They are shown to coincide with a direct linearization of standard Eulerian jump conditions. General interface conditions for displacement, stress, heat flux and temperature are given. As an example, these conditions are applied to the analysis of elastoacoustic wave propagation inside an inviscid fluid-filled pressurized duct. The combined effects of slip, prestress and initial flow are briefly discussed.

\section{2:40}

3aSAb9. Experimental and numerical investigation of the dynamics in spatial fluid-filled piping systems. Jan Herrmann (Institute of Applied and Experimental Mechanics, University of Stuttgart, Pfaffenwaldring 9, 70550 Stuttgart, Germany, herrmann@iam.uni-stuttgart.de), Thomas Haag (Institute of Applied and Experimental Mechanics, University of Stuttgart, Pfaffenwaldring 9, 70550 Stuttgart, Germany, thomasshaag@gmx .de), Lothar Gaul (Institute of Applied and Experimental Mechanics, University of Stuttgart, Pfaffenwaldring 9, 70550 Stuttgart, Germany, gaul@iam .uni-stuttgart.de)

Hydraulic piping systems, such as fluid-filled break and fuel pipes in automotive applications, undergo strong acoustic excitation due to pressure pulsations of pump and valve operation. By fluid-structure coupling the sound transmission within the pipe may lead to a structural excitation of other car components causing excessive noise levels or even structural failure. In order to obtain a complete and reliable understanding of the wave propagation and vibration phenomena in spatial piping systems, a test rig is presented, consisting of a pressure source and a fluid-filled break pipe with an attached target structure. With this experimental setup, it is possible to quantify the acoustic sound transmission and to examine the dynamic behavior by transfer functions. The experimental results are compared with harmonic and transient finite element simulations employing efficient model order reduction techniques for the fluid-structure coupled system. This research focuses on the identification of hydraulic resonances and the optimal mounting of the fluid-filled break pipe in order to minimize the structureborne sound induced on the target structure.

\section{3:00}

3aSAb10. Noise generation in an unstable boundary-layer flow over a flexible wall. Marie-Laure Gobert (IRENav, BP 600, 29240 Brest Armées, France, marie-laure.gobert@ecole-navale.fr), Uwe Ehrenstein (IRPHE, 49 rue Joliot-Curie, BP 146, 13384 Marseille Cedex 13, France, ehrenstein @irphe.univ-mrs.fr), Jacques Andre Astolfi (IRENav, BP 600, 29240 Brest Armées, France, astolfi@ecole-navale.fr), Patrick Bot (IRENav, BP 600, 29240 Brest Armées, France, bot@ecole-navale.fr), Jean Audet (Thales Underwater Systems, 525 route des Dolines, BP 157, 06903 Sophia Antipolis, France, Jean.Audet@fr.thalesgroup.com), Guillaume Oudot (DCNS Divison Navires Armés, Le Mourillon, BP 1306, 83076 Toulon cedex, France, guillaume.oudot@densgroup.com)

The boundary-layer flow, for instance along a sonar dome, gives rise to hydrodynamic noise due to the pressure fluctuations. The prediction of the resulting self-noise received by the sonar antenna is based on models, which in general take only partially into account the flexibility of the dome wall. The present work readdresses the problem of hydrodynamic noise, considering the geometrically simplified model of a two-dimensional unstable boundary-layer flow along an elastic plate with clamped ends. The incompressible Navier-Stokes equations are fully coupled to the elastic plate model and the system is numerically solved for various plate materials. The unstable flow dynamics is analyzed with respect to the wall properties. The Fourier-transformed stress tensor is then used in the framework of Lighthill's analogy to determine the generated radiative sound, emphasizing the effect of wall-flexibility. This work is supported by Thales Underwater System and DCNS. 


\title{
Session 3aSCa
}

\section{Speech Communication: Speech Prosody and How it Relates to Segmental Aspects of Speech}

\author{
Yi Xu, Cochair \\ University College London, Wolfson House, 4 Stephenson Way, London, NW1 2HE, UK \\ Hansjörg Mixdorff, Cochair \\ TFH Berlin University of Applied Sciences, Luxemburger Str. 10, Berlin, 13353, Germany
}

\section{Invited Papers}

\author{
8:00
}

3aSCa1. Segmental variability related to speech prosody - An articulatory-functional perspective. Yi Xu (University College London, Wolfson House, 4 Stephenson Way, NW12HE London, UK, yi.xu@ucl.ac.uk)

\begin{abstract}
Just like in the tonal aspect, variability abounds in the segmental aspect of speech. Here I argue that segmental variability, similar to supra-segmental variability, can be understood from an articulatory- functional perspective. That is, two independent sources of segmental variability co-exist: a) multiple layers of communicative functions, and b) articulatory mechanisms of their encoding. From an articulatory perspective, much of the segmental variability arises from the nature of the syllable as a product of generating time markers, namely, synchronized onsets of consonant, vowel, tone and phonation register at the start of each syllable as recurrent events that serve like clock ticks to enable time tracking by both speakers and listeners. Such time marking results in complete overlap of initial consonant and the following vowel, which is responsible for most of the variability known as coarticulation, and sequential offset of coda components in a syllable, making them susceptible to deletion and merger. From a functional perspective, segments as supralaryngeal articulatory movements encode not only lexical contrast, but also other functional contrasts such as focus, grouping, attitude, etc. Like the tonal aspect of speech, these extra-lexical information are probably encoded as modification of segmental targets that have been lexically assigned.
\end{abstract}

\section{8:20}

3aSCa2. Prosodic Variation: Understanding Scope, Categoricity, and Recursion in Speech Production and Perception. Dani Byrd (Dept of Linguisitcs, University of Southern California, 3601 Watt Way, GFS 301, Los Angeles, CA 90089-1693, USA, dbyrd@usc.edu), Jelena Krivokapic (Department of Linguistics, Yale University, 370 Temple St, Room 204, PO Box 208366, New Haven, CT 06520-8366, USA, jelena.krivokapic@yale.edu)

Prosodic structure refers to the level of linguistic structure above the segmental level, namely prominence and phrasal organization. We will examine both articulatory and perceptual characteristics of phrasal organization, and their interrelation. An experiment using articulatory movement tracking examines how far in time remotely from a boundary the effect of the boundary extends. In a second experiment (Krivokapic 2007), we examine how structural properties of a range of prosodic boundaries are reflected in perception-specifically, whether boundary perception is categorical or gradient. A third study considers whether prosodic junctures of the same category type (e.g, an intonational phrase boundary) can, when embedded, give rise in articulation to boundary signatures of measurably different strengths. That is, we evaluate the possibility of recursive prosodic structures of the kind that Ladd (1996) postulates. Taken together, these experiments inform us about structurally induced variability. They provide the opportunity to evaluate standard assumptions about prosodic structure such as the question of boundaries as symbolic or temporal events, categoricity, and strict layering. Throughout, we will consider how the pi-gesture framework (Byrd \& Saltzman 2003) offers theoretical insight regarding issues of prosodic representation and realization. [Supported by NIH]

\section{Contributed Papers}

\section{8:40}

3aSCa3. Temporal organization of prosodic and segmental features in spoken Japanese. Hiroya Fujisaki (University of Tokyo (Prof. emeritus), 3-31-12 Ebisu, Shibuya-ku, 150-0013 Tokyo, Japan, fujisaki@alum .mit.edu), Sumio Ohno (Tokyo University of Technology, 1404-1 Katakura, 192-0982 Hachioji, Japan, ohno@cc.teu.ac.jp)

It is apparent that prosodic and segmental features of speech must be temporally coordinated in order to produce a consistent and meaningful message. The precise mechanism for the coordination, however, has not been clear. The present study looks into this problem in the case of word accent in spoken Japanese. The speech material consisted of utterances of Japanese words that were identical in the word accent type, in the number of morae, in vowel constituents, but were different in consonantal constituents (including a 'null' consonant) at a certain intervocalic position. As for the prosodic features, the fundamental frequency contours were analyzed using the command-response model, and the onset and the offset of the extracted accent command were used as indices of prosodic timing. As for the segmental features, the formant frequency trajectories of the vowels were analyzed using another command-response model, which allowed extraction of the onset of the articulatory command for a vowel nucleus as an index of segmental timing. Comparison of the timings of these commands provides a means for quantitative analysis of the relationship between segmental and prosodic features of speech, and leads to an understanding of the underlying mechanism for their temporal coordination.

\section{9:00}

3aSCa4. Stress, focus, and the roots of segmental variation. Kenneth De Jong (Indiana University, 322 Memorial Hall, 1021 E. 3rd Street, Bloomington, IN 47405, USA, kdejong@indiana.edu)

de Jong (JASA, 1995) proposed a definition of linguistic stress as variation in the articulation of segmental material; stress is variation on the hypoto hyper-articulation dimension (Lindblom, 1990) localized to the syllable. 
This definition has suggested that the interaction of stress and segmental articulation will involve 1) linguistic conventionalization, since stress is a property of particular linguistic systems, 2) the structure of segmental contrasts, since hyper-articulation is driven by contrast enhancement, and 3) propensities in articulation, since hypo-articulation is driven by motor considerations. This paper reviews evidence for effects of linguistic conventionalization of stress. It further examines and compares the results of a number of experiments in segmental variation related to focus. Some dimen- sions of acoustic contrast, vowel quality, transition dynamics, and glottal timing, exhibit readily detectable and gradient variation as expected by a hyperarticulation model, and such variation is specific to the language's contrastive structure. Not all contrastive dimensions, however, are so affected, for example, noise associated with obstruent consonants. These patterns of results together suggest a functional interaction between the various speech gestures which may define a unit of speech at about the level of the syllable; the level which is the domain of expression of stress.

\section{Invited Papers}

\section{9:20}

3aSCa5. Close coupling or points of rendezvous? Connections between intonational events and the segmental grid. Hansjörg Mixdorff (TFH Berlin University of Applied Sciences, Luxemburger Str. 10, 13353 Berlin, Germany, mixdorff@tfh-berlin .de), Hartmut Pfitzinger (Christian-Albrechts-University, Leibnizstr. 10, 24118 Kiel, Germany, hpt@phonetik.uni-muenchen .de), Stefan Werner (University of Joensuu, PL111, 80101 Joensuu, Finland, stefan.werner@joensuu.fi)

The authors examine the connection between the fundamental frequency (f0) contour and the segmental grid, employing the Fujisaki model for decomposing an f0 contour into phrase and accent components. As shown in earlier work by the first author, accent commands are closely connected with the accented syllables of the constituent words of an utterance. They reflect the temporal alignment of intonational gestures by their on- and offset times, as well as the magnitude of the gestures by their amplitudes. Their timing relates to phonemic function, and the amplitudes are strongly correlated with the prominence of an accented syllable. In this study the authors attempt to model the intensity contour of an utterance as a concomitant of the articulatory gesture, namely by low-pass filtering pulsewise "syllable commands", yielding onset and offset points as well as amplitude values of the syllabic gestures and relate these data to the accent command data from the f0 tier. We examine the influence of phonemic distinctions on the alignment between the f0 contour and the segmental grid, as well as the influence of the intonational gesture on the realization of segments. The work is carried out on spontaneous American English speech data from the Buckeye corpus.

\section{9:40}

3aSCa6. Segmental effects on the prosody of voice quality. Hartmut Pfitzinger (Christian-Albrechts-University, Leibnizstr. 10, 24118 Kiel, Germany, hpt@phonetik.uni-muenchen.de)

Voice quality variability is due to supra-segmental influences but also to segmental factors like phoneme class, vowel quality, nasalization, airstream mechanism etc. These factors determine a rather unexplored micro-prosodic phenomenon: the phone-intrinsic voice quality which causes voice quality coarticulation and voice quality transitions in fluent speech. I subsume all these phenomena under the high-frequency components of prosody. Since high-frequency and low-frequency components (supra-segmentals) of voice quality prosody are superposed and thus encoded the main goal of this paper is to separate them and make both accessible to speech research. In 2003 a holistic voice quality parameter extractor was introduced by Mokhtari, Pfitzinger, and Ishi: It applies a principal components analysis to a database of glottal-flow waveforms for the purpose of later reconstructing and interpolating all underlying glottal-flow waveforms from just a few principal components. Recently, by applying this basic principle to a large corpus of manually segmented glottal-flow waveforms of 44 speakers I dramatically improved its applicability to any speech signals. The application of this version to a large speech database and subsequent high-pass and low-pass filtering of the resulting voice quality parameters yielded phone-intrinsic voice quality parameter sets as well as slowly varying meaningful voice quality parameter contours. 


\title{
Session 3aSCb
}

\section{Speech Communication: Speech Perception III}

\author{
Eva Reinisch, Chair \\ Max Planck Institute for Psycholinguistics, Postbus 310, Nijmegen, 6500AH, Netherlands
}

\section{Contributed Papers}

\section{8:40}

3aSCb1. Lexical stress information modulates the time-course of spoken-word recognition. Eva Reinisch (Max Planck Institute for Psycholinguistics, Postbus 310, 6500AH Nijmegen, Netherlands, eva.reinisch @mpi.nl), Alexandra Jesse (Max Planck Institute for Psycholinguistics, Postbus 310, 6500AH Nijmegen, Netherlands, alexandra.jesse @mpi.nl), James M. McQueen (Max Planck Institute for Psycholinguistics, Postbus 310, 6500AH Nijmegen, Netherlands, james.mcqueen@mpi.nl)

Segmental as well as suprasegmental information is used by Dutch listeners to recognize words. The time-course of the effect of suprasegmental stress information on spoken-word recognition was investigated by tracking Dutch listeners' looks to arrays of four printed words on a computer screen as they listened to spoken sentences. Each target was displayed along with a competitor that did not differ segmentally in its first two syllables but differed in stress placement (e.g., 'CENtimeter' and 'sentiMENT'; capitals marking stressed syllables). The listeners' eye-movements showed that suprasegmental stress information is used to recognize the target before distinct segmental information is available. Furthermore, the presence of initial-syllable stress affected the degree to which words compete for recognition: Targets with non-initial stress ('sentiMENT') suffered from stronger competition than initially stressed targets ('CENtimeter'). Follow-up experiments showed that initial-syllable duration, as a cue to lexical stress, is not interpreted dependent on the speaking rate of the preceding carrier sentence. This still held when other stress cues like pitch and amplitude were removed. Rather, the speaking rate of the preceding carrier affected the speed of word recognition globally, even though the speaking rate of the target itself was not altered.

\section{9:00}

3aSCb2. Using pause durations to discriminate between lexically ambiguous words and dialog acts in spontaneous speeech. Rajdip Dhillon (Dept. of Linguistics, Yale Univ., 370 Temple St., Rm. 204, New Haven, CT 06520, USA, rajdip.dhillon@yale.edu)

Using multiparty conversations conducted in spontaneous Scottish English speech, this study examines whether pause durations can be used to discriminate between lexically ambiguous words pertaining to different dia$\log$ acts and, more generally, whether pause durations signal different types of dialog acts. Dialog acts describe the pragmatic functions of utterances and their detection requires attention to pragmatic function, syntax, prosody, surrounding context, and interaction between speakers. In this study, the dialog acts examined are 'floor grabbers', which are used by speakers to gain attention or to interrupt a current speaker who has the floor, and 'acceptance/agreements', which express agreement to or acceptance of a previous speaker's question, proposal, or statement. Experiments 1 and 2 examine pause durations following the lexical items 'right' and 'good' respectively when used as 'floor grabbers' or 'acceptance/agreements'. Experiment 3 examines pause durations following 'floor grabbers' and 'acceptance/agreements', regardless of the lexical item. Results of all three experiments indicate that pauses following 'floor grabbers' are significantly longer than those following 'acceptance/agreements'. This study shows that pause durations are in fact a reliable means of discriminating between lexically ambiguous words and distinguishing dialog acts. The study also sheds light on how stable pause usage is in spontaneous speech.

\section{9:20}

3aSCb3. Identification of talkers speaking emotional speech. Kyung Wha Kim (Forensic Science Div, Supreme Prosecutors' Office, 706 Banporo, Seocho-gu, 137-730 Seoul, Republic of Korea, savoix@spo.go .kr), Patrick C. Wong (Northwestern University, 2240 Campus Drive, Evanston, IL 60208, USA, pwong@ northwestern.edu)

Identification of talkers speaking emotional speech is common in many communicative situations and forensic "voice line ups". However, emotional speech is auditorily and acoustically different from neutral speech, which may cause difficulty in talker identification. In this study, we examined how listeners identify different talkers speaking emotional or neutral voices, and how short-term training may benefit talker identification. Recordings were obtained from 10 male speakers speaking in angry and neutral voices. Within one session, listeners were first trained to identify the talkers, and their ability to generalize to untrained sentences spoken by the same talkers was tested. We found that while there was no significant difference in identifying neutral and angry voices, the ability to generalize to untrained sentences was more impaired with angry voices. The theoretical and forensic implications of these results are discussed. [Work supported by NIH and $\mathrm{NSF}]$

\section{9:40}

3aSCb4. An fMRI-based structural equation model for natural language processing shows age-dependent changes in brain connectivity. Scott K. Holland (Cincinnati Children's Hospital Medical Center, Imaging Research Center, 3333 Burnet Ave, Cincinnati, OH 45229, USA, scott.holland@cchmc.org), Prasanna Karunanayaka (Cincinnati Children’s Hospital Medical Center, Imaging Research Center, 3333 Burnet Ave, Cincinnati, OH 45229, USA, KAR4RP@cchmc.org), Elena J. Plante (Univ. Arizona, Dept. Speech and Hearing Sciences, P.O. Box 210071, Tucson, AZ 85721-0071, USA, eplante@U.Arizona.EDU), Vincent J. Schmithorst (Cincinnati Children's Hospital Medical Center, Imaging Research Center, 3333 Burnet Ave, Cincinnati, OH 45229, USA, Vince .Schmithorst@cchmc.org)

Structural Equation Modeling (SEM) or path analysis is a multivariate analytic tool that is used to test hypothesis about causal influences among measured or latent variables. When applied to functional neuroimaging (fMRI) data, SEM combines interregional covariance and neuroanatomy to investigate brain connectivity and the dynamic flow of information across neural networks. We have investigate Linear SEM or LSEM as a first step in estimating connection strengths from fMRI data in children during acoustic stimulation with stories in English. An average LSEM was constructed based on the concatenated fMRI data from $\mathrm{N}=313$ children, age 5 to 18 , listening to stories. Time courses from the fMRI data is used as input to the LSEM as computed in AMOS (v.5.1, SmallWaters, Corp. Chicago, IL) to yield an average model for the entire group. The LSEM path coefficients were examined between brain regions involved in auditory speech perception and language processing. Connectivities estimated by LSEM are significantly age dependent in some brain areas, while the connectivity coefficient between other brain regions is not a function of age. 


\title{
Session 3aSCc
}

\section{Speech Communication: Speech Articulation II}

\author{
Yves Laprie, Chair \\ LORIA CNRS, 615 rue du Jardin Botanique, Bâtiment C, Villers-lès-Nancy, 54600, France
}

\section{Contributed Papers}

\section{0:40}

3aSCc1. Modeling formant pattern in fricative-vowel sequences. Martine Toda (CNRS \& ENST, 46, rue Barrault, 75634 Paris, France, martinetoda@yahoo.co.jp), Shinji Maeda (CNRS \& ENST, 46, rue Barrault, 75634 Paris, France, maeda@tsi.enst.fr)

MRI data indicate that there are two subject-dependent articulatory patterns in French post-alveolar sibilant fricative /'integral of'/. Each type of articulations can correspond to the Polish fricatives /'curled c'/ and /'underdotted s' $\%$ on the basis of their articulatory similarity: palatalized for the former, and the presence of a sublingual cavity as well as lip protrusion for the latter. The F2 transitions, in part, reflect the acoustic contrast between these Polish fricatives, which exhibit a higher locus in the palatalized /'curled c'/ than in /'underdotted s'/. However, no systematic transitional characteristic could be found in French. We hypothesize that the subjectspecific formant-cavity affiliation in the adjacent vowel /a/ and/or the difference of scale in vocal tract lengths manifest as the acoustic variation in French, whereas those VT morphological differences are articulatorily compensated in Polish to maintain the distinctiveness of these two post-alveolar fricatives. In order to assess this hypothesis, the F-patterns along /a 'integral of' a/ sequences are simulated for various vocal tract configurations. In calculations, the two different types of the fricative articulations are combined with the vocal-tract area functions appropriate for $/ \mathrm{a} / \mathrm{but}$ different in shape and in size to taken into account for female and male speakers. The results will be discussed.

\section{1:00}

3aSCc2. Diffraction effects on speech phonemes during phonation. Vijayakumar V (Multimedia University, FOSEE, Jalan Air Keroh Lama, 75450 Melaka, Malaysia, vijaya@mmu.edu.my), Eswaran C (Multimedia University, Faculty of Information Technology, Cyberjaya Campus, 63100 Cyberjaya, Malaysia, eswaran@mmu.edu.my)

In this paper we will show how the ventricular fold modifies speech phonemes and also causes the shimmering of speech. Diffraction of sound waves at the ventricular fold which can be considered as a circular aperture, can modify the vocalized glottal sound spectrum reaching the pharyngx. Thus during phonation, speech phoneme spectra are essentially enveloped by Bessel functions. Since Bessel beams are known to be nondiffracting, this step ensures that the phonemes do not undergo further diffraction effects which can destroy the unique harmonic structure of the phonemes. Note that the wavelengths of sound waves and the dimensions of the human vocal tract appendages are of the same order of magnitude which mean that diffraction should be commonplace in the vocal tract if not for the initial packaging of the speech phoneme at the ventricular fold.

\section{1:20}

3aSCc3. Evaluation of phonetic constraints in acoustic-to-articulatory inversion. Yves Laprie (LORIA CNRS, 615 rue du Jardin Botanique, Bâtiment C, 54600 Villers-lès-Nancy, France, Yves.Laprie@loria.fr), Blaise Potard (LORIA CNRS, 615 rue du Jardin Botanique, Bâtiment C, 54600 Villers-lès-Nancy, France, Blaise.Potard@loria.fr), Anne Bonneau (LORIA
CNRS, 615 rue du Jardin Botanique, Bâtiment C, 54600 Villers-lès-Nancy, France, Anne.Bonneau@loria.fr)

The objective is to evaluate phonetic constraints intended to guarantee the phonetic realism of vocal tract shapes generated by an articulatory model, i.e. here that of Maeda. The constraints are based on the relation between vocal tract shapes allowed for a vowel and formants frequencies of the vowel. They are speaker independent since they are derived from standard phonetic knowledge. We used them to reduce the number of solutions in acoustic-to-articulatory inversion. The impact of constraints has been investigated by comparing vocal tract shapes recovered by inverting five French vowels $/ \mathrm{i}, \mathrm{e}, \mathrm{a}, \mathrm{u}, \mathrm{y} /$ to those obtained by outlining articulator contours in X-ray images. To that purpose we used X-ray images (used by Maeda to elaborate his articulatory model) with the corresponding speech signal. The evaluation shows that unrealistic vocal tract shapes are penalized by constraints, that vocal tract shapes recovered through inversion are in very good agreement with X-ray images and that relevant articulatory variability is recovered when considering all vocal tract shapes corresponding to one vowel. These constraints thus complement the articulatory model very efficiently. Additionally, the inversion framework complemented by phonetic constraints is an efficient way of investigating the acoustic properties of an articulatory model.

\section{1:40}

3aSCc4. Acoustic-to-articulatory inversion of fricatives. Farid Feïz (LORIA CNRS, 615 rue du Jardin Botanique, Bâtiment C, 54600 Villers-lès-Nancy, France, Farid.Feiz@loria.fr), Blaise Potard (LORIA CNRS, 615 rue du Jardin Botanique, Bâtiment C, 54600 Villers-lès-Nancy, France, Blaise.Potard@loria.fr), Yves Laprie (LORIA CNRS, 615 rue du Jardin Botanique, Bâtiment C, 54600 Villers-lès-Nancy, France, Yves.Laprie @loria.fr)

The objective is to recover the vocal tract shape dynamics from the speech signal of vowels and fricatives. The method relies on the analysisby-synthesis paradigm and is an extension of the method proposed by Ouni and Laprie which exploits a hypercubic articulatory table to represent the synthesis facet, i.e. Maeda's articulatory model. The first major modification is the use of parallelepiped instead of cubes. The new construction strategy only subdivides the articulatory space in the articulatory direction which gives rise to the strongest non-linearities. This enables a substantial reduction of the table size. The second major modification is the inversion of fricative sounds. In addition to the articulatory parameters the relative location of the noise source downstream the constriction is taken into account. This gives rise to three different articulatory codebooks, each corresponding to the relative position of the source with respect to the main constriction. This new inversion method has been evaluated on VCV sequences. It turns out that the correct vocal tract dynamics is recovered even if the constriction area is slightly underestimated. Input data are the first three formant frequencies but MFCC coefficients are now investigated since they render the high frequency region of fricative spectra better. 


\title{
Session 3aSCd
}

\section{Speech Communication: Prosody I}

\author{
Volker Dellwo, Chair \\ UCL, Wolfson House, 4, Stephenson Way, London, NWI 2HE, UK
}

\section{Contributed Papers}

\section{0:40}

3aSCd1. Quantifying rhythm in running speech. Tristie Ross (Dept. of Linguistics, UCSD, 9500 Gilman Drive \#0108, La Jolla, 92093-0108, USA, tross@ling.ucsd.edu), Naja Ferjan (Dept. of Linguistics, UCSD, 9500 Gilman Drive \#0108, La Jolla, 92093-0108, USA, naja@ling .ucsd.edu), Amalia Arvaniti (Dept. of Linguistics, UCSD, 9500 Gilman Drive \#0108, La Jolla, CA 92093-0108, USA, amalia@ling.ucsd.edu)

In the past decade several metrics that reflect the variability of vocalic and consonantal intervals in speech have been used to quantify the impressionistic division of languages into stress- and syllable-timed. Although all such metrics successfully separate prototypical languages, such as stresstimed English and syllable-timed Spanish, their results for other languages are less clear. The problem is related to the limited datasets used, which consist of either a small number of sentences per language elicited from several speakers, or longer stretches of speech elicited from one speaker per language. Here we elicited short sentences, story reading and spontaneous speech from several speakers of stress-timed English, syllable-timed Spanish, Korean, a hitherto unclassified language, and Greek, which has shown to resist classification. Our results show that different metrics yield different classifications for some languages, like Greek, while scores for the same language differ depending on speaking style. Taken all together these results cast doubt on the robustness and usefulness of the popular metrics and suggest that alternative ways of conceiving of speech rhythm that do not rely exclusively on timing but take relative prominence into account may ultimately be more successful in explaining speech rhythm.

\section{1:00}

3aSCd2. Temporal Organization of the Compounds in Standard Chinese. Eric Zee (Phonetics Lab, Dept. of Chinese, Translation and Linguistics, City University of Hong Kong, 83 Tat Chee Avenue, Kowloon Hong Kong, Hong Kong, eric.zee@ cityu.edu.hk), Wai-Sum Lee (Phonetics Lab, Dept. of Chinese, Translation and Linguistics, City University of Hong Kong, 83 Tat Chee Avenue, Kowloon Hong Kong, Hong Kong, w.s.lee @ cityu.edu.hk)

The study investigates the temporal organization of the bisyllabic, trisyllabic and quadrisyllabic compounds in Standard Chinese. Results of the analysis of the speech data from six speakers show that (a) the rime durations, the durations of the syllable-initial voiceless fricatives and the VOT of the syllable-initial voiceless aspirated stops and affricates are conditioned by the position of the component syllable in the compounds; (b) the rime duration decreases as the component syllable moves from the final toward the initial position in the compounds; (c) the mean duration of the rimes is longer in the final position than in the initial and medial positions; (d) the voiceless fricatives and the voiceless aspirated stops and affricates are shorter in the medial position than in the initial and final positions; (e) the cross-speaker variation is less in the rime duration than in the duration of the voiceless fricatives and VOT of the voiceless aspirated stops and affricates; and (f) no temporal patterns have been observed in the voiceless unaspirated stops and affricates. The results will be discussed in terms of articulatory programming and timing (Kozhevnikov and Chistovich, 1965; Allen, 1975). A temporal model of the compound production in Standard Chinese will be proposed.

\section{1:20}

3aSCd3. Second language rhythm and rhythm metrics. Naja Ferjan (Dept. of Linguistics, UCSD, 9500 Gilman Drive \#0108, La Jolla,
92093-0108, USA, naja@ling.ucsd.edu),Tristie Ross(Dept. of Linguistics, UCSD, 9500 Gilman Drive \#0108, La Jolla, 92093-0108, USA, tross@ling .ucsd.edu), Amalia Arvaniti (Dept. of Linguistics, UCSD, 9500 Gilman Drive \#0108, La Jolla, CA 92093-0108, USA, amalia@ ling.ucsd.edu)

Several metrics that reflect the variability of vocalic and consonantal intervals in speech have been shown to successfully discriminate between the rhythm of first and second language (L2) speakers producing short sentences of a given language. Here we elicited running read speech from native speakers of English, German and Italian speaking English. We hypothesized that the scores of German L2 speakers would be closer to the scores of native English speakers, since both English and German are stress-timed, and that the scores of Italian L2 speakers would exhibit greater differences from native English scores, since Italian is stress-timed. Our results show instead that the scores of some German speakers are less close to those of the native English speakers than the scores of some Italian speakers, a difference that cannot be attributed to fluency, proficiency level, or length of exposure to English. It is possible that these results reflect over-compensation of some sort by some L2 speakers, though a more likely explanation is that the popular metrics used to quantify speech rhythm may be unreliable or at least not sufficiently robust to be used with L2 data. Reasons why this may be so for L2 speech in particular are discussed.

\section{$11: 40$}

3aSCd4. Influences of language typical speech rate on the perception of speech rhythm. Volker Dellwo (UCL, Wolfson House, 4, Stephenson Way, NW12HE London, UK, v.dellwo@ucl.ac.uk)

Human listeners can distinguish languages of different rhythmic classes (e.g. stress- and syllable-timed languages). The present study investigated the role of speech rate in this process. In experiment I, speech rate measurements (syllables/second) of read speech were performed in two stress-timed (English, German) and two syllable-timed languages (French, Italian). The results revealed that speech rate distinguishes as reliable between rhythmic class as previously proposed correlates of speech rhythm $(\% \mathrm{~V}$, VarcoC and nPVI). For a perceptual task (experiment II) sentences from French and German were delexicalized by turning consonantal intervals into white noise and vocalic intervals into complex periodic tones. 18 listeners rated the regularity of the noise-tone sequences on a 13 point scale. The results revealed that listeners rated French stimuli as significantly more regular than English stimuli. It was further shown that the rate of noise-tone sequences was the best predictor for the regularity rating (faster sequences were perceived as more regular) compared to standard rhythm measures $(\% \mathrm{~V}$, VarcoC and $\mathrm{nPVI}$ ). Both experiments together show that a) syllable-timed languages are produced faster than stress-timed languages and b) listeners' perception of speech rhythm is highly influenced by these rate differences.

\section{2:00}

3aSCd5. Computing the fundamental frequency variation spectrum in conversational spoken dialogue systems. Kornel Laskowski (Carnegie Mellon University, 407 South Craig Street, Pittsburgh, PA 15213, USA, kornel@cs.cmu.edu), Matthias Wölfel (Universität Karlsruhe, am Fasanengarten 5, 76131 Karlsruhe, Germany, wolfel@ira.uka.de), Mattias 
Heldner(KTH, Lindstedtsvägen 24, SE-100 44 Stockholm, Sweden, mattias@speech.kth.se), Jens Edlund (KTH, Lindstedtsvägen 24, SE-100 44 Stockholm, Sweden, edlund@ speech.kth.se)

Continuous modeling of intonation in natural speech has long been hampered by a focus on modeling pitch, of which several normative aspects are particularly problematic. The latter include, among others, the fact that pitch is undefined in unvoiced segments, that its absolute magnitude is speakerspecific, and that its robust estimation and modeling, at a particular point in time, rely on a patchwork of long-time stability heuristics. In the present work, we continue our analysis of the fundamental frequency variation (FFV) spectrum, a recently proposed instantaneous, continuous, vectorvalued representation of pitch variation, which is obtained by comparing the harmonic structure of the frequency magnitude spectra of the left and right half of an analysis frame. We analyze the sensitivity of a task-specific error rate in a conversational spoken dialogue system to the specific definition of the left and right halves of a frame, resulting in operational recommendations regarding the framing policy and window shape.

12:20

3aSCd6. A longitudinal study on vocal aging - Changes in F0, jitter, shimmer and glottal noise. Hideki Kasuya (International Univ. of Health and Welfare, 2600-1 Kita-kanemaru, 324-8501 Otawara, Japan, kasuyah
@ snow.ucatv.ne.jp),Hajime Yoshida(Yoshida Clinic, West 3, South 6, 0968585 Nayoro, Japan, nort3381@ @orth.hokkai.net), Hiroki Mori (Fac. of Engr., Utsunomiya Univ., 7-1-2 Yoto, 321-8585 Utsunomiya, Japan, hiroki @ speech-lab.org), Hiroshi Kido (Tohoku Inst. of Technology, 35-1 Yagiyama Kasumicho, Taihaku-ku, 982-8557 Sendai, Japan, kidoh@ tohtech .ac.jp)

A longitudinal study on vocal aging was conducted for voicing source parameters: fundamental frequency (F0), jitter (period perturbation quotient, PPQ), shimmer (amplitude perturbation quotient, APQ) and glottal noise (normalized noise energy in a high frequency band, $\mathrm{NNEb).} \mathrm{Acoustic} \mathrm{analy-}$ ses were made on the sustained phonations of the Japanese vowel /a/ of 20 males and 38 females with no laryngeal disease, which were recorded once every year over periods ranging from 11 to 19 years. The acoustic parameters measured were subjected to statistical regression analysis, where the longitudinal change of the parameters was evaluated by the t-test. Two males $(10 \%)$ and 12 females $(32 \%)$ showed significant falling in F0 $(\mathrm{P}<0$ $.05)$ but no significant rising was observed. Five males (25\%) and 12 females $(32 \%)$ displayed a significant increase in APQ $(\mathrm{P}<0.05)$, whereas only six females $(16 \%)$ and no male revealed significant changes in PPQ $(\mathrm{P}<0.05)$. Increase in NNEb was significant for seven males $(35 \%)$ and 14 females $(37 \%)(\mathrm{P}<0.05)$. We conclude that 1$) \mathrm{F} 0$ falling is a more emblematic tendency of female vocal aging than male, 2) shimmer is a more observable indication of vocal aging than jitter, and 3) glottal noise in a high frequency region tends to increase with age.

WEDNESDAY MORNING, 2 JULY 2008

ROOM 343, 8:00 TO 10:40 A.M.

\title{
Session 3aSPa
}

\section{Signal Processing in Acoustics and Physical Acoustics: Biomedical Applications of Time-Reversal II}

\author{
Oleg A. Sapozhnikov, Cochair \\ Center for Industrial and Medical Ultrasound, Applied Physics Lab., University of Washington, 1013 NE 40th St., Seattle, WA \\ 98105, USA
}

Mickael Tanter, Cochair

Laboratoire Ondes et Acoustique, ESPCI, Université Paris 7, CNRS, 10 rue Vauquelin, Paris, 75005, France

\section{Invited Papers}

8:00

3aSPa1. Time-Reversal Acoustics in Biomedical Engineering. Mathias Fink (Laboratoire Ondes et Acoustique, ESPCI, Université Paris 7, CNRS, 10 rue Vauquelin, 75005 Paris, France, mathias.fink@espci.fr), Gabriel Montaldo (Laboratoire Ondes et Acoustique, ESPCI, Université Paris 7, CNRS, 10 rue Vauquelin, 75005 Paris, France, gabriel.montaldo@espci.fr), Mickael Tanter (Laboratoire Ondes et Acoustique, ESPCI, Université Paris 7, CNRS, 10 rue Vauquelin, 75005 Paris, France, michael.tanter@espci.fr)

Time-reversal is a very powerful method for focusing through complex and heterogeneous media and shows very promising results in biomedical applications. In this presentation, we review some of the main applications investigated during the past decade. An iterative implementation of the time-reversal process allows tracking gallstones in real time during lithotripsy treatments. In this application domain, a smart exploitation of the reverberations in solid waveguides permits the focusing of high amplitude ultrasonic shock waves with a small number of transducers. Because time reversal is able to correct the strong distortions induced by the skull bone on ultrasonic propagation, this adaptive focusing technique allows brain ultrasonic therapy through the skull bone. Finally, time-reversal focusing guided by speckle noise can be implemented to correct distortion in medical ultrasonic imaging and recent results will be presented.

$8: 20$

3aSPa2. A comparison of planar projection and time reversal techniques. Gregory Clement (Harvard Medical School, Focused Ultrasound Lab - BWH Radiology, 221 Longwood Ave RM 521, Boston, MA 02115, USA, gclement@hms.harvard.edu)

Planar projection methods, such as the angular spectrum approach, have been shown to rapidly relate fields between two planes in space/time, with only a single measurement plane needed to characterize an entire field. Variants of the method have been used for medical transducer characterization, as well as for HIFU treatment planning. Presently, a potential advantage of the approach is considered: Operating in the temporal frequency domain, backward planer projection (i.e. "space reversal") is invariant with respect to 
the spatial dimension, even in the presence of anomalous dispersion. This ability can be contrasted with time reversal, which is violated in the presence of the absorption term. Time reversal is compared with planar back-projection under a varying set of conditions, including the presence of dispersive and nonlinear homogeneous and heterogeneous media. The specific case of a simulated impulsive signal from a $30-\mathrm{cm}$ diameter piston radiator is directed through a $50-\mathrm{cm}$ section of a tissue-like medium $(\mathrm{B} / \mathrm{A}=5$ to $7, \mathrm{c}=1480$ to $1600 \mathrm{~m} / \mathrm{s}, \rho=0.9$ to $1.1 \mathrm{gm} / \mathrm{cm} 3, \alpha=0.5$ to $2 \mathrm{db} / \mathrm{cm} / \mathrm{MHz}$ ) over a range of pressures. Ability of backward projection to restore initiating signals is verified, provided that significant signal levels of all relevant frequencies can be reached at the initial plane.

\section{8:40}

3aSPa3. Nonlinear imaging of isoechogenic phantoms using selective phase conjugation of acoustic harmonics. Vladimir L. Preobrazhensky (IEMN, UMR CNRS 8520, avenue Poincaré, BP 60069, 59652 Villeneuve d'Ascq, France, vladimir .preobrajenski@iemn.univ-lille1.fr), Philippe Pernod (IEMN, UMR CNRS 8520, avenue Poincaré, BP 60069, 59652 Villeneuve d'Ascq, France, philippe.pernod@iemn.univ-lille1.fr), Leonid M. Krutyansky (WRC-GPI-RAS LEMAC, Vavilova street, 38, 119991 Moscow, Russian Federation, krut@orc.ru)

Selective phase conjugation of acoustic harmonics generated by an intense focused probing wave is applied for imaging of isoechogenic phantoms non detectable by the linear echography. The principle of imaging is based on the difference between the nonlinear properties of a phantom and its surrounding medium. As shown by theoretical modelling the analysis of the phase conjugate second harmonic $2 \omega$ of the probing wave provides an image contrast comparable with the relative difference between the nonlinear parameters of the phantom and its background. Second harmonic generation by the phase conjugate wave $2 \omega$ during its back propagation to the source of the probing wave provides an image contrast at the frequency $4 \omega$ even stronger than the difference of nonlinear parameters. These theoretical conclusions are confirmed by the experimental measurements carried out using $10 \mathrm{MHz}$ supercritical parametric phase conjugator. The objects were prepared with methanol water solutions of concentrations $20 \%$ and $60 \%$. The solutions filling either a cavity in the agar gel or an acoustically transparent container immersed in water represented isoechogenic phantoms with weaker and stronger nonlinearities relatively to water. The contrast values of $2 \omega$-images were measured as $-14 \%$ and $+10 \%$ respectively while for $4 \omega$-images the contrast achieved $-45 \%$ and $+37 \%$.

\section{9:00}

3aSPa4. Ultrasonic transducers for imaging and therapy based on time-reversal principles. Armen Sarvazyan (Artann Laboratories, 1753 Linvale-Harbourton, Lambertville, NJ 08350, USA, armen@artannlabs.com)

Focusing of ultrasonic waves is a fundamental aspect of most of the medical applications of ultrasound. There are three approaches in focusing acoustic energy: geometrical, using concave transducers and acoustic lenses, electronic, using phased arrays, and timereversal (TR), which allows both spatial and temporal concentration of acoustic energy. The aim of this talk is to make comparative analysis of advantages and disadvantages of ultrasound focusing techniques, demonstrate unique capabilities of TR focusing in medical imaging, surgery and therapy. Examples of embodiments of ultrasonic focusing transducers utilizing TR method for various biomedical applications will be presented. TR ultrasonic transducers are comprised of a reverberation chamber, which could be either an aluminum block or a water filled volume of irregular shape, and one or several piezotransducers attached to or incorporated in the reverberator. Optimization of the TR transducers for a particular application is considered, including the geometry of the TR reverberator, shape, type and dimensions of the piezotransducers. Unique abilities of TR transducers to steer the focal spot in 3D, produce pulses with arbitrary waveforms in a wide frequency band and generate focal regions of arbitrary predefined shapes using simple means such as single channel system with one piezoelement will be demonstrated.

3aSPa5. Doppler ultrasonography using wave phase conjugation. Yuri Pyl'Nov (Moscow Institute of Radio Engineering, Electronics \& Automation, Vernadsky prosp., 78, 119454 Moscow, Russian Federation, pylnov@yandex.ru), Nikolay Smagin (Moscow Institute of Radio Engineering, Electronics \& Automation, Vernadsky prosp., 78, 119454 Moscow, Russian Federation, pylnov@yandex.ru), Philippe Pernod (IEMN, UMR CNRS 8520, avenue Poincaré, BP 60069, 59652 Villeneuve d'Ascq, France, philippe.pernod@iemn.univ-lille1.fr), Vladimir L. Preobrazhensky (IEMN, UMR CNRS 8520, avenue Poincaré, BP 60069, 59652 Villeneuve d'Ascq, France, vladimir.preobrajenski@iemn.univ-lille1.fr)

Ultrasound wave phase conjugation (WPC) is an efficient tool for velocimetry of flows due to its sensibility to the break of time reversal invariance of acoustic fields in moving media. The presence of flow leads to the non compensated Doppler phase shift of the backward phase conjugate wave relatively to the primary probing wave. This phase shift provides detection, diagnostics and velocimetry of flows and does not require the presence of any scatterers in a flow. Furthermore the phase shift of phase conjugate wave can be used in liquid flowmeters. As it was shown in previous works the sensitivity of liquid flowmeters using phase conjugate ultrasonic waves essentially increases by using nonlinear effects appearing during propagation of phase conjugate waves in a moving liquid. In the present paper we report the theoretical and experimental results on imaging of flow velocity distributions in tubes and phantoms of blood vessel. The scanning auto-confocal system based on parametric ultrasonic wave phase conjugator at $10 \mathrm{MHz}$ operation frequency is used in the experiments. The preliminary results of experimental measurements of velocity of vascular blood flow in soft tissues in vitro are discussed. 
3aSPa6. Guiding of HIFU beams towards optical contrast agents by time-reversal of photoacoustic waves. Emmanuel Bossy (Laboratoire Photons et Matière, ESPCI/CNRS, 10 rue Vauquelin, 75231 Paris Cedex 05, France, emmanuel .bossy@espci.fr), Jean-François Aubry (Laboratoire Ondes et Acoustique, ESPCI, Université Paris 7, CNRS, 10 rue Vauquelin, 75005 Paris, France, jean-francois.aubry@espci.fr), Arik R. Funke (Laboratoire Photons et Matière, ESPCI/CNRS, 10 rue Vauquelin, 75231 Paris Cedex 05, France, arik.funke@espci.fr), Mathias Fink (Laboratoire Ondes et Acoustique, ESPCI, Université Paris 7, CNRS, 10 rue Vauquelin, 75005 Paris, France, mathias.fink@espci.fr), Albert-Claude Boccara (Laboratoire Photons et Matière, ESPCI/CNRS, 10 rue Vauquelin, 75231 Paris Cedex 05, France, boccara@optique.espci.fr)

This presentation will discuss the opportunity to use photoacoustics to guide high-intensity focused ultrasound (HIFU) in biological tissue. The photoacoustic effect consists in the emission of sound by an illuminated optically absorbing region, based on the thermoelastic expansion following the absorption of a light pulse. In the near-infrared range ( $700 \mathrm{~nm}$ to $900 \mathrm{~nm}$ ), the absorption of light in biological tissue is relatively low, and multiply scattered photons can travel as deep as a few centimetres in tissue. Photoacoustic waves can therefore be generated deep in tissue, and have recently been used in vivo to perform passive acoustical imaging of optical absorbing features, such as blood vessels. We recently demonstrated on tissue-mimicking phantoms that photoacoustics could be coupled to timereversal to automatically focus ultrasound towards an optical absorber [Bossy et al., APL 89(18), 2006]. We propose to further develop this approach to automatically guide HIFU therapy beams towards a targeted region labelled with an optically absorbing contrast agent. This presentation will discuss various aspects of such an approach, based on modelling and experimental results.

\section{Contributed Papers}

10:00

3aSPa7. Time Reversal Acoustic focusing using layered membranes structure. Yegor Sinelnikov (ProRhythm, 105 Comac St., Ronkonkoma, NY 11779, USA, yegor@prorhythm.com), Andrey Vedernikov (ProRhythm, 105 Comac St., Ronkonkoma, NY 11779, USA, vedernikov@prorhythm.com), Alexander Sutin (Stevens Institute of Technology, Castle Point on Hudson, Hoboken, NJ 07030, USA, asutin@stevens.edu), Armen Sarvazyan (Artann Laboratories, 1753 Linvale-Harbourton, Lambertville, NJ 08350, USA, armen@artannlabs .com)

In the recent years numerous Time Reversal Acoustic Focusing Systems (TRA FS) employing various reverberation chambers ("acoustic cavities") accumulating acoustic energy in time were described in the literature. We propose an alternative reverberator composed of a liquid-filled volume comprising a series of spatially distributed membranes with varying thickness in the range from one quarter to half wavelength of ultrasound. Random spatial variation of ultrasound reflection from such membranes leads to randomization of acoustic field that highly improve Time Reversal focusing of the system. A theoretical model of reflections from nonuniform membranes is suggested to evaluate spatial and temporal characteristics of TRA focusing formed as a result of numerous reflections and reverberation of ultrasound propagating though the plurality of membranes. The dependence of focal structure and focused waveform was investigated for suggested structure and their focusing efficiency has been compared with that of the conventional reverberators. In the experiment, the membrane were made of Polyethylene Terephthalate or Nylon thermoformed films. Ultrasonic signals were radiated by transducers with frequency in the range from 3 to $10 \mathrm{MHz}$.
Experiment demonstrated effective TRA focusing by membrane arrangement that can be used for ultrasound focusing and steering in various minimally-invasive biomedical applications.

\section{0:20}

3aSPa8. Time Reversed Elastic Waves within Soft Solids. Stefan Catheline (LOA, Université Paris 7 Denis Diderot, ESPCI, 10 rue Vauquelin, 75005 Paris, France, stefan.catheline@espci.fr), Carlos Negreira (Science University of Montevideo, 11400 Montevideo, Uruguay, carlosn@fisica.edu.uy), Nicolas Benech (Science University of Montevideo, 11400 Montevideo, Uruguay, nbenech@ fisica.edu.uy), Javier Brum (Science University of Montevideo, 11400 Montevideo, Uruguay, jbrum@ fisica.edu.uy)

When a scalar far-field wave is time reversed from the surface of a cavity, it starts to converge toward its initial point source location, then collapses and finally diverges. Without near field evanescent waves, the symmetric focus spot is limited by the Rayleigh criterion. We present an experimental observation of a time-reversal vectorial wave in the volume of a soft solid. The elastic field is measured using the transient elastography technique. It is observed that the time reversed far field wave collapses and gives birth to near field effects. Numerical computation based on elastodynamic Green's functions in a time-reversal cavity confirms and completes the experimental conclusions: the time symmetry with respect to collapse time is broken and the Rayleigh criterion is direction dependant; the spatial collapse is larger in the direction of the point body force than in the perpendicular direction. The authors anticipate that the method can be used for shear wave beam forming in soft tissues as well as to tissue elasticity assessment. 


\title{
Session 3aSPb
}

\section{Signal Processing in Acoustics: Filter Design, Detection, and Estimation I}

\author{
Michael Roan, Cochair \\ Virginia Tech, 917 Village Way North, Blacksburg, VA 24060, USA \\ Jorge E. Quijano, Cochair \\ NEAR Lab-Portland State University, 1900 SW 4th Avenue, Suite 160, Portland, OR 97201, USA
}

\section{Contributed Papers}

\section{1:00}

3aSPb1. Wavelet filter bank based wide-band audio coder. Jan Nováček (Czech Technical University, Faculty of Electrical Engineering, Technicka 2, 16627 Prague, Czech Republic, novacj1@fel.cvut.cz)

New system for wide-band audio signals compression is presented. The system is based on a two stage wavelet filter bank principle. Special psychoacoustic model was designed for the system. Data compression is nowadays very topical issue. Due to the development of information technologies more sophisticated compression algorithms may be designed. Most of the present compression systems, e.g. MPEG, ATRAC or Vorbis, use a simple filter bank or DCT transformation. Both methods suffer from the origin of undesirable artefacts which are disturbing for the sound perception. Wavelet filter bank is used to suppress these artefacts and to increase data compression. Frequency dependant windowing function is used for improve of frequency resolution at low frequencies along with faster response at high frequencies. Wavelet transformation based coding algorithm can improve temporal masking effects over conventional perceptual coding algorithms for data compression.

\section{1:20}

3aSPb2. Investigation of a novel nonlinear detector of weak acoustic signals in noise. Julian L. Deeks (Institute of Sound and Vibration, Univ. of Southampton, University Road, Highfield, SO17 1BJ Southampton, UK, jd1@isvr.soton.ac.uk), Paul R. White (Institute of Sound and Vibration, Univ. of Southampton, University Road, Highfield, SO17 1BJ Southampton, UK, prw@isvr.soton.ac.uk)

Recent work has identified and explored a method of detecting weak signals in Gaussian noise, based on the response of a nonlinear system to small input perturbations. The technique is applicable to the detection of weak underwater acoustic signals in unwanted ambient sea noise. Much of the research to date has been carried out using a forced Duffing oscillator as the nonlinear system, with parameters selected so as to place the system at the point of transition from a chaotic state to a stable periodic state. The additive input perturbation, most commonly comprising a simulated single frequency embedded in Gaussian noise with a very low input SNR, has been found to initiate the transition as indicated by the appearance of a stable trajectory in phase space. The attendant increase in amplitude at the output of the detector, at or near to the signal frequency, constitutes a detection of that weak signal. This paper presents a quantitative investigation of the technique as applied to underwater acoustic signals (real and simulated), using receiver operating characteristics (ROC) as a measure of its performance. Both the Duffing system, and a more novel version of the technique using a forced Lorenz system, are investigated.

\section{$11: 40$}

3aSPb3. Comparison of non linear system identification methods : the example of non linear propagation of acoustic waves in ducts. Antonin Novak (Laboratoire d'Acoustique de l'Université du Maine, Avenue Olivier Messiaen, 72085 Le Mans, France, antonin.novak.etu@univ
-lemans.fr),Laurent Simon(LAUM, CNRS, Université du Maine, Lab. d'Acoustique Université du Maine, UMR CNRS 6613, 72085 Le Mans Cedex 9, France, Laurent.Simon@univ-lemans.fr), Joël Gilbert (Laboratoire d'Acoustique de l'Université du Maine, Avenue Olivier Messiaen, 72085 Le Mans, France, joel.gilbert@univ-lemans.fr), Pierrick Lotton (Laboratoire d'Acoustique de l'Université du Maine, Avenue Olivier Messiaen, 72085 Le Mans, France, Pierrick.Lotton@univ-lemans.fr), Frantisek Kadlec (CVUT, Department of Radioelectronic, Technická 2, 16627 Praha 6, Czech Republic, kadlec@fel.cvut.cz)

The weakly non linear propagation of travelling acoustic waves in ducts is a well known problem leading to approximated analytical solutions. From an experimental point of view, the classical way for estimating the non linear parameters of propagation is to generate sine waves and to analyse the higher order harmonics as a function of the amplitude and the frequency of the excitation. In this work, new methods for estimating the non linear parameters of propagation are developed and compared to the sine excitation based method. The excitation signals associated to these new methods can be stationary noise or logarithmic chirps. For these excitation signals, the data processing is based on Multiple Input Single Output (MISO) direct path method. The comparison is made in terms of signal to noise ratio robustness and computation time. Experimental and theoretical results are also compared. We particularly show that a measurement using only one logarithmic chirp allows estimating accurate results for a broad band of amplitudes and frequencies. Associated computational time is then drastically reduced.

\section{2:00}

3aSPb4. The Use of Mel Cepstral Coefficients and Markov Models for the Automatic Identification, Classification and Sequence Modelling of Salient Sound Events Occurring During Tennis Matches. Gordon J. Hunter (Kingston University, Faculty of Computing, Information Systems and Mathematics, Penrhyn Road, KT1 2EE Kingston-upon-Thames, UK, G.Hunter@kingston.ac.uk), Krzysztof Zienowicz (Kingston University, Faculty of Computing, Information Systems and Mathematics, Penrhyn Road, KT1 2EE Kingston-upon-Thames, UK, zienowicz@btinternet .com), Ahmed I. Shihab (Kingston University, Faculty of Computing, Information Systems and Mathematics, Penrhyn Road, KT1 2EE Kingstonupon-Thames, UK, ais@kingston.ac.uk)

Some significant events in sports matches occur too quickly to be detected by conventional video. Audio signals, normally sampled at a much higher rate, provide a way to detect such short events. Here, we employ methods used in automatic speech recognition - templates of Mel Frequency Cepstral Coefficients (MFCCs) over several adjacent time windows, with Principal Components Analysis (PCA) - to identify and classify sound events : different tennis strokes, ball bounces, echos, speech and applause, occurring during tennis matches. Excellent success rates were achieved for both event detection ( $97.74 \%$ ) and correct classification (on average 98.64 $\%$ across all classes) of 1504 sound events in the available recordings. The successful classification rate varied between classes but no class had a success rate below $94 \%$. This could be valuable to spectators, officials and coaches in tennis and other sports - including cricket, baseball and golf - or 
to make video games (such as the Nintendo Wii) more realistic. We also model sequences of these events in both discrete (event oriented) and continuous time domains, using Markov and other models, to give our system predictive as well as reactive capability, help identify "unusual" or "unexpected" salient sounds and hopefully improve the correct classification rate for the classes where the performance was weakest.

\section{2:20}

3aSPb5. 3D Gabor analysis of transient space-time waves. Loic Martinez (ECIME Université de Cergy-Pontoise, rue d'Eragny, 95031 Neuville-sur-Oise, France, loic.martinez@u-cergy.fr), Nicolas Wilkie-Chancellier (ECIME Université de Cergy-Pontoise, rue d'Eragny, 95031 Neuville-sur-Oise, France, Nicolas.Wilkie-Chancellier@u-cergy .fr), Christ Glorieux (Lab. ATF, Katholieke Universiteit Leuven, Celestijnenlaan 200D, B-3001 Leuven, Belgium, christ.glorieux@fys .kuleuven.be)

Laser generation/detection methods allow the investigation of ultrasonic transient phenomena in both space and time dimensions. Used for the experimental investigation of surface wave propagation along a two dimensional medium, laser ultrasonics leads to three dimensional (3D) space-time signal collections. In order to extract the wave propagation information, the classical high resolution signal processing methods or 3D Fourier Transforms are not suitable to identify the transient and local aspect of wave propagation and mode conversion. In order to quantify these transient aspects in the Space-Time-Wave number-frequency domains, the 3D Gabor transform and some other suitable methods are presented. Their potential for the identification of the local and transient complex wave numbers is illustrated with the propagation of Lamb waves on a plane limited plate. The experimental results are in good agreement with the numerical simulations.

\section{$12: 40$}

3aSPb6. Computer learning method for scattering junction identification. Adam Kestian (New York University, 77 Park Ave., apt 1214, Hoboken, NJ 07030, USA, apk240@nyu.edu), Agnieszka Roginska (New York University, 35 West Fourth St., Room 777, New York, NY 10012, USA, roginska@nyu.edu)

Acoustic reflectometry is a non-invasive, time-domain method of identifying the geometry of an acoustical space. A sound pulse is injected into a space and the resulting impulse response details particular changes of impedance, which is a result of a cross-sectional area change or an elbow/Tintersection. Each cause of reflection, known as a scattering junction, has a distinct reflection contour. Previous works were able to identify these scattering junctions via algorithms that attempt to extract particular contours from the impulse response. In the present study, the prominent reflections of the space are observed, isolated, and then compared to a training database of all possible scattering junctions. This method eliminates the necessity to create a contour identification algorithm, as scattering junctions are defined based on its most similar neighbors in the training database. Preliminary results suggest that this computer-learning algorithm can successfully identify reflection contours of a space with varying cross-sectional areas from those that were stored in the training database, which suggests that this method could be a more efficient and versatile alternative to previous identification processes.

\title{
Session 3aUWa
}

\section{Underwater Acoustics, Acoustical Oceanography, and ECUA: Impact of Environmental Variability on Mid-Frequency Sonar Performance}

\author{
Dajun Tang, Cochair \\ Applied Physics Laboratory, University of Washington, 1013 NE 40th St, Seattle, WA 98105, USA \\ Paul C. Hines, Cochair \\ Defence R\&D Canada - Atlantic, P.O. Box 1012, Dartmouth, NS B2Y3Z7, Canada \\ Peter Louring Nielsen, Cochair \\ NURC, Viale S. Bartolomeo 400, La Spezia, 19126, Italy
}

Invited Papers

8:00

3aUWa1. Operational Impacts of the Environment on Mid-Frequency Navy Sonar Systems In Shallow Water. Marcus M. Speckhahn (Planning Systems Inc., 3990 Old Town Avenue, Cabrillo Plaza, Suite A101, San Diego, CA 92110-2930, USA, marcus.speckhahn.ctr@navy.mil), Stephen C. Lingsch (Commander Naval Meteorology and Oceanography Command, 1100 Balch Boulevard, Stennis Space Center, MS 39529, USA, stephen.lingsch@navy.mil), Josette P. Fabre (Naval Research Laboratory, Code 7180, Stennis Space Center, MS 39529, USA, josie.fabre@nrlssc.navy.mil), Michael T. Garr (Naval Undersea Warfare Center, Code 1521, 1176 Howell Street, Newport, RI 02841, USA, michael.garr@navy.mil), Sandra K. Wetzel-Smith (Naval Mine and AntiSubmarine Warfare Command, 32444 Echo Lane, Suite 300, San Diego, CA 92147, USA, skw@spawar.navy.mil)

The coastal oceans represent a challenging domain for mid-frequency maritime Anti-Submarine Warfare (ASW) sonar systems to operate effectively. Information on the advantages and disadvantages of, as well as general tactical employment criteria for the prevalent types of active mid-frequency sonar systems found in the world's navies is provided. Operational performance of these systems, which include hull-mounted sonars, monostatic buoys and multistatic active systems, as well as dipping sonars, is always affected by shallow water environmental parameters. These acoustic and oceanographic factors include frequent bottom interactions of the transmitted acoustic energy, highly variable sound speed and noise fields, strong coastal currents and high levels of acoustic clutter. Accurate and timely four-dimensional sensor performance predictions and optimization recommendations can mitigate some of the negative impact of 
the environment on sonar detection ranges. We discuss the trade-offs and assumptions made by modern computer-based tactical decision aids to deliver the most accurate environmental information balanced by the need to deliver these data in tactical time frames useful to the ASW mission planner and sonar operator at sea. We will also present thoughts on the quantification of acoustic uncertainty in support of ASW operations and the need for high-fidelity active sonar training systems.

\section{$8: 20$}

3aUWa2. Quantifying ocean acoustic sensitivity to environmental uncertainty/variability. Stan E. Dosso (University of Victoria, School of Earth and Ocean Sciences, Victoria, BC V8W 3P6, Canada, sdosso@uvic.ca)

It is well known that acoustic data used in sonar applications are strongly influenced by physical parameters of the ocean environment. Environmental sensitivity studies are often based on a qualitative comparison of acoustic fields computed for a reference environmental model and for a perturbed model in which one or more parameters have been changed. The acoustic-field perturbation generally includes a component representing a spatial shift of the field (i.e., local field structure remains coherent, but shifts in range and/or depth) and a component representing a change to the shifted field. Hence, the field change at a fixed point can indicate high sensitivity in cases where the field structure changes little but is simply offset, which can conflict with an intuitive understanding of sensitivity. This paper quantifies sensitivity rigorously in terms of a statistical measure of the relative uncertainty of acoustic fields or transmission loss due to realistic uncertainty/variability in environmental parameters. Further, accounting for field shifts can provide a more meaningful sensitivity measure for many applications. The sensitivity of acoustic fields to the state of knowledge of the soundspeed profile, bathymetry, and seabed geoacoustic parameters is examined for both range-independent and range-dependent cases. [Funded by DRDC-Atlantic Rapid Environmental Assessment Project.]

\section{8:40}

3aUWa3. Tools and techniques to measure environmental parameters that improve mid-frequency sonar performance predictions. John C. Osler (Defence R\&D Canada - Atlantic, P.O. Box 1012, Dartmouth, NS B2Y3Z7, Canada, john.osler@drdc-rddc.gc.ca), Sean Pecknold (Defence R\&D Canada - Atlantic, P.O. Box 1012, Dartmouth, NS B2Y3Z7, Canada, sean.pecknold@drdc-rddc.gc.ca), Paul C. Hines (Defence R\&D Canada - Atlantic, P.O. Box 1012, Dartmouth, NS B2Y3Z7, Canada, Paul.Hines@drdc-rddc.gc.ca)

The performance of mid-frequency active sonar systems in littoral waters depends upon several environmental factors and their variability in space and time. These factors include seabed scattering strength, bottom loss, and the water column sound speed profiles. DRDC Atlantic has developed experimental apparatus and techniques to measure these environmental parameters in order to enhance the accuracy of sonar performance estimates in the mid-frequency band, 1 to $10 \mathrm{kHz}$. Experiments have been conducted during sea-trials on the Scotian Shelf and Strataform area on the New Jersey Shelf. In some instances, these experiments have been in conjunction with international research partners to provide an inter-comparison between experimental techniques and/or a comparison of rapid environmental assessment techniques with more traditional, and time-consuming, methods. In this talk, we will demonstrate that predictions of sonar system performance improve when the environmental parameters obtained with these techniques are used, rather than parameters from archival sources.

\section{Contributed Papers}

\section{9:00}

3aUWa4. A comparison of methods for approximating acoustic uncertainty in underwater sound channels. Kevin R. James (Univ. of Michigan, 2010 Lay Auto Lab, Ann Arbor, MI 48109, USA, krjames @ umich.edu), David R. Dowling (Univ. of Michigan, 2212 G. G. Brown Laboratory, Ann Arbor, MI 48109, USA, drd@umich.edu)

The accuracy and reliability of acoustic field calculations are often determined by uncertainty in environmental parameters. There are currently several approaches for predicting calculated-acoustic-field uncertainty arising from environmental uncertainties. This presentation outlines the current development of a method for predicting acoustic uncertainty based on correlations between variations in an uncertain parameter and spatial shifts within a calculated acoustic field. The results of this technique are compared with several other modern methods for predicting acoustic uncertainty such as direct sampling of environmental parameters, linearization and higherorder finite difference approaches, the adjoint method for approximating derivatives of the acoustic field with respect to environmental parameters, and polynomial chaos methods. The advantages and limitations of each technique are presented for range-independent shallow-ocean sound channels at nominal ranges of 1 to $10 \mathrm{~km}$ and frequencies from $100 \mathrm{~Hz}$ to $1 \mathrm{kHz}$. Comparisons of accuracy, computational speed, and applicability to particular uncertain parameters and field prediction routines are provided. Emphasis is placed on identifying the types of sound channel properties and environmental uncertainties for which each technique performs most reliably, as well as identifying situations for which current techniques are not yet adequate [Sponsored by the Office of Naval Research, Code 321OA].

\section{9:20}

3aUWa5. Fluctuation of mid-frequency propagation in shallow water. Dajun Tang (Applied Physics Laboratory, University of Washington, 1013 NE 40th St, Seattle, WA 98105, USA, djtang@apl.washington.edu), Frank S. Henyey (Applied Physics Laboratory, University of Washington, 1013 NE 40th St, Seattle, WA 98105, USA, frank@ apl.washington.edu), Kevin L. Williams (Applied Physics Laboratory, University of Washington, 1013 NE 40th St, Seattle, WA 98105, USA, williams@apl.washington.edu), Daniel Rouseff (Applied Physics Laboratory, University of Washington, 1013 NE 40th St, Seattle, WA 98105, USA, rouseff@apl.washington.edu), Peter H. Dahl (Applied Physics Laboratory, University of Washington, 1013 NE 40th St, Seattle, WA 98105, USA, dahl@apl.washington.edu), Zhongkang Wang (Hangzhou Applied Acoustics Research Institute, PO Box 1249, 310012 Hangzhou, China, zkwang@apl.washington.edu), Jorge E. Quijano (NEAR Lab-Portland State University, 1900 SW 4th Avenue, Suite 160, Portland, OR 97201, USA, jorgeq@pdx.edu), Jee Woong Choi (Dept. of Environmental Marine Sciences, Hanyang Univ., 1271, Sa-3-dong, Sangrok-gu, 426-791 Ansan, Republic of Korea, choijw@ hanyang.ac.kr)

As part of the ONR-sponsored SW06 experiment, mid-frequency sound propagation was measured at ranges $1-10 \mathrm{~km}$ in the frequency band of 2-10 $\mathrm{kHz}$ in August, 2006. The water depth is $80 \mathrm{~m}$ and the source depth is $30 \mathrm{~m}$, close to the minimum of a duct with a thermocline above and a warm salty water below. The receivers are clustered into two groups, one at $25 \mathrm{~m}$ depth, the other at $50 \mathrm{~m}$. The region has active internal wave activity during this time. Because the source is near the axis of the sound channel, it is observed that propagation is dominated by trapped modes and behaves similar to 
sound propagation in a deep water duct. Amplitude fluctuations and crossfrequency correlations are estimated. The scintillation index as a function of frequency and bandwidth is calculated.

\section{9:40}

3aUWa6. Active Tracking using the Invariance Extended Kalman Filter (IEKF). Lisa M. Zurk (NEAR Lab-Portland State University, 1900 SW 4th Avenue, Suite 160, Portland, OR 97201, USA, zurk1@cecs .pdx.edu), Chensong He (NEAR Lab-Portland State University, 1900 SW 4th Avenue, Suite 160, Portland, OR 97201, USA, zurk1@pdx.edu), Jorge E. Quijano (NEAR Lab-Portland State University, 1900 SW 4th Avenue, Suite 160, Portland, OR 97201, USA, jorgeq@pdx.edu), Christian Hempel (1176 Howell Street, Newport, RI 02841, USA, HempelCG@Npt.NUWC.Navy .Mil)

The pressure received from a moving source in a shallow water channel has a frequency dependent structure that is imposed by the propagation characteristics of the channel. While an exact description of this structure requires fine knowledge of the channel properties (including bottom composition), an approximate relationship which utilizes the so-called waveguide invariant can be used to describe the intensity variations as a function of frequency versus range. This relationship has been extensively explored and utilized for application to passive sonar systems. Recent work has demonstrated that a similar structure, called the active invariance, may be present in active sonar. In this paper the active invariance relationship is incorporated into an extended Kalman filter (EKF) to create a new tracking formulation called the Invariance EKF (IEKF). The IEKF differs from the conventional formulation by incorporating the time-dependent frequency content into both the state space representation and the update equations, where the relationship between target position and frequency is given by the active invariance expression. Performance of the IEKF relative to conventional approaches is explored with normal mode simulations and bistatic data collected in the Maltese channel. Sponsored by ONR-321US

\section{0:00}

3aUWa7. Comparison of sonar transmission models at mid-frequency for synthetic and real environments. Adrian D. Jones (Defence Science and Technology Organisation, P.O Box 1500, 5111 Edinburgh, Australia, bearjones@chariot.net.au), Janice S. Sendt (Thales Australia, 274 Victoria Road, 2116 Rydalmere, Australia, Janice.Sendt@au.thalesgroup.com), Alec J. Duncan (Curtin University of Technology, Centre for Marine Sci \& Tech, GPO Box U1987, 6845 Perth, WA, Australia, A.J.Duncan@curtin.edu .au), Paul A. Clarke (Defence Science and Technology Organisation, P.O Box 1500, 5111 Edinburgh, Australia, Paul.Clarke@dsto.defence.gov .au), Zhiyong Zhang (Defence Science and Technology Organisation, P.O Box 1500, 5111 Edinburgh, Australia, Yong.Zhang@dsto.defence.gov .au), Han Vu (Defence Science and Technology Organisation, P.O Box 1500, 5111 Edinburgh, Australia, Han.Vu@dsto.defence.gov.au)

The suitability of sonar transmission loss models for application to the mid-range of acoustic frequencies has been studied by comparing the outputs from a number of models. Emphasis has been placed upon the requirements for models for circumstances relevant to both continental shelf and deep ocean zones within the Australian region. In particular, the ability to describe transmission in surface ducted environments for a deep ocean, and in a shallow ocean with both ducted and downward refracting environments has been considered. For the deep ocean case, a synthetic environment was selected, and the ability to describe duct trapping was examined. For the shallow ocean case, scenarios selected for comparison correspond with ocean tracks for which environmental parameters had been collected and received sound pressure time series measured. The subject transmission loss models are from a variety of types. To enable the most valid comparison, input parameters have been matched across models to the fullest extent. This paper presents the state of progress of this work, including a comparison of models amongst themselves and, for continental shelf scenarios, against measured transmission loss.

\section{0:20-10:40 Break}

10:40

3aUWa8. In ocean evaluation of low frequency active sonar systems. Karl Thomas Hjelmervik (FFI, Postboks 115, 3191 Horten, Norway, kth @ffi.no), Geir Helge Sandsmark (FFI, Postboks 115, 3191 Horten, Norway, ges@ffi.no)

Sonar performance measurements in the sea are always affected by uncontrollable and/or uncertain environmental conditions as sound speed variations, bottom topography or the acoustic properties of the sea floor. This paper presents a method to determine a sonar - target geometry which minimizes the uncertainty in target signal excess due to environmental variability. An acoustic model is used to estimate the signal excess for a large number of sound speed profiles measured in the relevant area. The results are compared seeking a target range and depth where the estimated signal excess is robust with respect to the variation of sound speed profiles to be expected in the actual area. Results from sea trials will be presented as well as simulated examples used to demonstrate the robustness or sensitivity of the signal excess to environmental changes at different test geometries.

\section{1:00}

3aUWa9. Effects of volume and boundary variability on the statistics of received signal frequency. Jeremy Joseph (ARL Penn State, PO Box 30, State College, PA 16804, USA, jmj269@psu.edu), Richard Lee Culver (ARL Penn State, PO Box 30, State College, PA 16804, USA, rlc5@psu.edu), Colin W. Jemmott (ARL Penn State, PO Box 30, State College, PA 16804, USA, cwj112@psu.edu)

The overall goal of our research is to incorporate statistical knowledge of received passive signal variability and uncertainty into the signal processing. To accomplish this goal we need to better understand the sources of this variability, which are due in part to propagation through the ocean channel. Propagation introduces significant variation in received signal parameters through rough surface and bottom scattering as well as volume inhomogeneities. This talk focuses on the variability introduced by these sources and its effect on the statistics of the frequency spectrum of the received signal. We consider low frequencies, for which theoretical predictions are compared with PE-based simulation results. Work supported by ONR Undersea Signal Processing Code 321US.

\section{1:20}

3aUWa10. Modeling higher order statistics of shallow water reverberation. Kevin D. Lepage (Naval Research Laboratory, 4555 Overlook Ave SW, Washington, DC 20375, USA, kevin.lepage@nrl.navy.mil)

The modeling of the higher moments of shallow water reverberation is addressed for problems where non-Rayleigh reverberation is caused by scattering from rough boundaries with non-Gaussian height distributions. Expressions for the first three spatial moments of exponentially heightdistributed roughness are derived for both Gaussian and exponential correlation functions corresponding to Gaussian or von-Karman second order spectra. The bi and tri-spectra for these roughness distributions are also derived, and differences are highlighted between these results and the results that are obtained for Gaussian height-distributed higher order spectra. Finally results for the higher moments of reverberation pressure are derived for exponentially height-distributed bottom roughness with the Gaussian spatial correlation function in the presence of multipath as a function of time, bandwidth, and correlation length scale. [Work supported by ONR]

\section{1:40}

3aUWa11. Computational Predictions of sonar performance based on full wave acoustic propagation modeling. Michael Wolfson (Univ. of Washington, Applied Physics Lab., 101340 St NE, Seattle, WA 98105, USA, wolfson@apl.washington.edu)

For fishery acoustics, sonars offer the advantage of inferring the biomass of a given ocean volume at a lower operational cost of traditional echosounders, but wave guide effects which induce multi-pathing yield unknown variances in bio-mass estimates. A sonar performance model has recently been developed to assist in understanding this variance based on the para- 
axial approximation to the acoustic wave equation. The model takes into account the geometry of the sonar deployment (source depth, transmit/receive beam patterns, pulse shape) and the acoustic environment (water column and geo-acoustic bottom) and creates a data base of two-way transfer functions for a chosen set of range-depth cells. Then, given a hypothetical aggregation or school of fish, each with its own target strength, as well as noise and reverberation levels, one can create intensity maps which can be used to perform sensitivity studies to ascertain the performance characteristics of a fishery sonar. This talk will discuss the theoretical development of the model, its constraints, and provide an example which highlights its potential for future work. The example will be in a shallow water $(100 \mathrm{~m})$ environment using acoustic frequencies of around 10 kilohertz for imaging aggregations of pelagic fish. The Naval Ocean Partnership Program is gratefully acknowledged for this work.

\section{2:00}

3aUWa12. Temporal and spatial coherence of low frequency acoustic modes in shallow water. Harry Deferrari (Univ. of Miami, 4600 Rickenbacker Causeway, Miami, FL 33149, USA, hdeferrari@rsmas .miami.edu), Hien B. Nguyen (Univ. of Miami, 4600 Rickenbacker Causeway, Miami, FL 33149, USA, hnguyen@rsmas.miami.edu), Jennifer Whylie (Univ. of Miami, 4600 Rickenbacker Causeway, Miami, FL 33149, USA, jwhylie@rsmas.miami.edu)

Three recently collected shallow water acoustic propagation data sets have common experimental parameters of fixed sources with multiple frequency m-sequence transmissions, HLA and VLA receptions, and detailed measurements of the ocean acoustic environments. In each case, broad band signals allow the separation of modes by arrival time and the computation of spatial and temporal coherency for individual modes of propagation thus eliminating the effects of multipath interference. Here, coherency estimates are compared for several ranges from 10 to $80 \mathrm{Km}$ and depths of 80 to 250 $\mathrm{m}$, frequencies from 100 to $1600 \mathrm{~Hz}$ and for a wide range of potential energy levels of the internal wave field. There are a number of consistent findings. Low order modes are more coherent than high order modes in both space and time. Low frequency signals respond deterministically and in pro- portion to internal wave fluctuations whereas higher frequencies are randomized and have saturated statistics. In between, phase wrapping occurs. The number of bottom interactions is shown to be a critical factor in coherence especially for frequencies of $800 \mathrm{~Hz}$ and above. At very long ranges out of plane arrivals introduce multipath interference even for single mode reception.

\section{2:20}

3aUWa13. Effects of internal waves on acoustic coherent communications during SW06. Aijun Song (University of Delaware, College of Marine and Earth Studies, S. College Street, Newark, DE 19716, USA, ajsong@udel.edu), Mohsen Badiey (University of Delaware, College of Marine and Earth Studies, S. College Street, Newark, DE 19716, USA, badiey@udel.edu), Arthur Newhall (Woods Hole Oceanographic Institution, 98 Water Street, Bigelow 203A, MS-11, Woods Hole, MA 02543, USA, anewhall@whoi.edu), James F. Lynch (Woods Hole Oceanographic Institution, 98 Water Street, Bigelow 203A, MS-11, Woods Hole, MA 02543, USA, jlynch@whoi.edu), Harry Deferrari (Univ. of Miami, 4600 Rickenbacker Causeway, Miami, FL 33149, USA, hdeferrari@rsmas.miami .edu)

During the SW06 experiment conducted east off the New Jersey continental shelf from July to September of 2006, internal wave surface expression and water temperature profiles were recorded when phase shift keying signals (PSK) were transmitted to study internal wave effects on coherent underwater acoustic communications at low to mid frequency bands. The collected communications data are processed using time reversal multichannel combining followed by a decision feedback equalizer. Due to the presence of internal waves, the acoustic channels show a time-varying property. Continuous channel updates prior to time reversal combining are required to combat fast channel fluctuations. The communications performance in terms of output signal-to-noise ratio exhibits significant change due to the internal waves. Further, the internal wave effects on the performance of coherent acoustic communications exhibit a frequency- and depth- dependency. The choices of receiver parameters also can be affected by the presence of internal waves.

\title{
Session 3aUWb
}

\section{Underwater Acoustics and ECUA: Sonar System and Transducer Calibration Methodology III}

\author{
Kenneth G. Foote, Cochair \\ Woods Hole Oceanographic Institution, Woods Hole, MA 02543, USA \\ Stephen P. Robinson, Cochair \\ National Physical Laboratory, Hampton Road, Teddington, TW11 OLW, UK
}

\section{Invited Paper}

3aUWb1. Calibration of wideband systems, need for a standard. Manell E. Zakharia (French Naval Acadamey, BP 600, 29240 Brest-Armees, France, manell.zakharia@ecole-navale.fr)

Although wide band sonar system are becoming more and more popular, there is no unified standard method for their calibration. Most of the wideband systems are calibrated at multiple frequencies using single frequency methods. Although such calibrations give correct figures, these figures do not correspond to the real use of the systems and do not display properly the problems encountered while using such systems such as signal deformation due to the directivity pattern. For instance, the zeroes of directivity patterns that correspond to destructive interference at one frequency are spatially smeared even for a band of $10 \%$ and appear as spectral variations. We will show two examples of such calibration with increasing difficulty. The first case considers a wideband sounder covering two 
octaves $(20-80 \mathrm{kHz})$ and the second one a parametric array (primaries around $100 \mathrm{kHz}$ and parametric generation from 10 to $20 \mathrm{kHz}$ ). In the second case, the difficulty is increased due to the factor of about ten between the primary and the secondary frequencies that are also associated to important level difference (about $40 \mathrm{~dB}$ ). It requires a high linearity constraint of the whole calibration chain.

\section{Contributed Paper}

\section{8:20}

3aUWb2. Calibration of broadband sonar systems using multiple standard targets. Philip Atkins (University of Birmingham, Department of Electronic, Electrical and Computer Engineering, Edgbaston, B15 2TT Birmingham, UK, p.r.atkins@bham.ac.uk), David T. Francis (University of Birmingham, Department of Electronic, Electrical and Computer Engineering, Edgbaston, B15 2TT Birmingham, UK, francdti@adf.bham .ac.uk), Kenneth G. Foote (Woods Hole Oceanographic Institution, Woods Hole, MA 02543, USA, kfoote@whoi.edu)

A seven-octave active sonar system spanning the nominal frequency range $25-3200 \mathrm{kHz}$ was deployed in Norwegian waters for the purpose of measuring the acoustic scattering characteristics of a range of marine organisms. This system transmitted linear frequency-modulated (LFM) signals in order to achieve good range resolution and to obtain spectral information on resolved targets. Total system performance was variously measured in situ and ex situ, depending on the particular octave band, using standard-target spheres. This enabled the frequency response of the entire system to be determined and the sidelobe level of the matched-filter receiver to be reduced. The effects of the deep nulls encountered in the backscattered spectrum of target spheres were partially reduced by using a string of up to six spheres of different sizes and material properties. Typical results will be presented showing that such calibration procedures are sensitive the relative alignment of the sonar-target and to sound-speed profile changes over the length of the string. [This work originated with EU RTD support through contract MAS3-CT95-0031.]

\section{Invited Paper}

\section{8:40}

3aUWb3. Measuring the equivalent beam angle of echo sounders with split-beam transducers. Lars Nonboe Andersen (Simrad, P.O. Box 111, 3191 Horten, Norway, lars.nonboe.andersen@simrad.com), Egil Ona (Institute of Marine Research, P.O.Box 1870 Nordnes, 5817 Bergen, Norway, egil.ona@imr.no), Geir Pedersen (Institute of Marine Research, P.O.Box 1870 Nordnes, 5817 Bergen, Norway, geir.pedersen@imr.no), Frank Tichy (Simrad, P.O. Box 111, 3191 Horten, Norway, frank.tichy@kongsberg.com) , Even Borten Lunde (Simrad, P.O. Box 111, 3191 Horten, Norway, even.borten.lunde@kongsberg.com)

When trying to measure the numerical density of marine animal populations with scientific echo sounders, several technical system parameters are determined through a standard-target calibration. The acoustic scattering properties of standard targets are known $a$ priori. In a typical standard-target calibration the system gain and the transducer beam pattern are estimated. The system gain parameter is used both when measuring volume backscattering strength and when measuring target strength. The calibrated beam pattern is currently used only for target strength estimation. However, measurements of mean volume backscattering strength also require knowledge of the effective sampling volume of the acoustic beam, namely the equivalent beam angle. This parameter is typically not determined during an ordinary echo sounder calibration. A method for measuring the equivalent beam angle and the transducer angle sensitivity of a specific split-beam transducer is described, and results from exemplary calibrations are presented. Changes in the calibration parameters as a function of environmental parameters, especially the sound speed in water, are discussed.

\section{Contributed Paper}

\section{9:00}

3aUWb4. Multibeam sonar calibration techniques. Christian De Moustier (UNH, Center for Coastal and Ocean Mapping, Chase Ocean Engineering Lab, 24 Colovos Road, Durham, NH 03824, USA, cpm@ieee .org), Barbara J. Kraft (UNH, Center for Coastal and Ocean Mapping, Chase Ocean Engineering Lab, 24 Colovos Road, Durham, NH 03824, USA, bjkraft@cisunix.unh.edu), Glenn McGillicuddy (UNH, Center for Coastal and Ocean Mapping, Chase Ocean Engineering Lab, 24 Colovos Road, Durham, NH 03824, USA, glennm@ccom.unh.edu)

The acoustic calibration facility at the University of New Hampshire's Chase Ocean Engineering Laboratory has been used to calibrate several transducer arrays from multibeam sonars that operate at $100 \mathrm{kHz}$ and above. In each case, we mount the transducer array on a rotating colum and mea- sure the array's transmit and receive characteristics with a reference transducers leased from the Naval Undersea Warfare Center, Newport RI. Our rotation angles are known to within $0.2^{\circ}$ and our acoustic calibration measurements are repeatable to within $0.5 \mathrm{~dB}$. After adjusting for measured impedance mismatches between the elements of the array, we determine the actual element separation as the ratio of physical spacing over acoustic wavelength, and we use this result to estimate the beam pointing accuracy of the sonar. The beamforming gain is then estimated for all beam directions from broadside to the full range of steering angles used by the sonar. This work is motivated by research efforts to obtain calibrated acoustic backscatter measurements from multibeam echo-sounder that are used for seafloor characterization applications or for detection and identification of targets in the water column.

\section{Invited Papers}

3aUWb5. A comparison of hydrophone near-field scans and optical techniques for characterising high frequency sonar transducers. Victor F. Humphrey (Institute of Sound and Vibration, Univ. of Southampton, University Road, Highfield, SO17 1BJ Southampton, UK, vh@isvr.soton.ac.uk), Stephen P. Robinson (National Physical Laboratory, Hampton Road, TW11 OLW Teddington, UK, Stephen.Robinson@npl.co.uk), Peter D. Theobald (National Physical Laboratory, Hampton Road, TW11 OLW Teddington, UK, pdt@npl.co.uk), Gary Hayman (National Physical Laboratory, Hampton Road, TW11 OLW Teddington, UK, Gary.Hayman@npl.co.uk), Martin P. Cooling (National Physical Laboratory, Hampton Road, TW11 OLW Teddington, UK, mc@isvr .soton.ac.uk) 
Two potential methods of fully characterising the response of high frequency sonar transducers and arrays operating in the frequency range $100 \mathrm{kHz}$ to $500 \mathrm{kHz}$ are compared. In the first approach two-dimensional planar scans, with a spatial resolution of better than half a wavelength, are performed in the acoustic near-field using a small probe hydrophone. The measured two-dimensional data are propagated numerically using a Fourier Transform method to predict the far-field response. Alternatively the data can be back-propagated to re-construct the pressure distribution at the source, a powerful diagnostic technique which can identify defects in transducers and array elements. The second approach uses a scanning laser vibrometer to measure the velocity of the transducer surface; with the resulting velocity data also being used to predict the far-field response by numerical propagation. The two approaches are compared for a number of devices. Comparison of the propagated hydrophone near-field scan data with direct measurements at these ranges shows very good agreement, indicating the usefulness of the method for deriving far-field transducer responses from near-field measurements in laboratory tanks. The potential limitations introduced to the optical approach by the acousto-optic effect are discussed.

\section{9:40}

3aUWb6. Acousto-optic tomography for mapping of high-frequency sonar fields. Peter D. Theobald (National Physical Laboratory, Hampton Road, TW11 OLW Teddington, UK, pdt@npl.co.uk), Stephen P. Robinson (National Physical Laboratory, Hampton Road, TW11 OLW Teddington, UK, Stephen.Robinson@npl.co.uk), Gary Hayman (National Physical Laboratory, Hampton Road, TW11 OLW Teddington, UK, Gary.Hayman@npl.co.uk), Triantafillos Koukoulas (National Physical Laboratory, Hampton Road, TW11 OLW Teddington, UK, triantafillos.koukoulas@npl.co.uk)

This paper presents an acousto-optic method for tomographic mapping of acoustic fields generated by high-frequency sonar array transducers. The method uses a laser interferometer to measure the integrated refractive index change across the propagating acoustic wave generated by the transducer. An interferometer being a displacement or velocity measuring device, interprets this rate of change of optical path length as a displacement or velocity. Obtaining a series of these projections by scanning the laser beam or the transducer for a number of rotation angles of the transducer allow a two-dimensional plane of the acoustic field to be reconstructed using the techniques commonly used in X-ray Computed Tomography. Acousto-optic tomographic measurement results are presented for a 95 by $95 \mathrm{~mm}, 400 \mathrm{kHz}$ 1-3 composite, 4 element sonar array transducer and are compared to conventional planar hydrophone scans obtained using a $1.5 \mathrm{~mm}$ probe hydrophone. The optical method allows measurement of the acoustic field without perturbing the field being measured, which can occur when using the planar hydrophone scanning method. Different tomographic geometries are considered, including parallel beam and fan-beam methods, with the advantages and disadvantages of each being discussed.

\section{Contributed Paper}

\section{0:00}

3aUWb7. Prediction for the acoustic field of a high frequency cylindrical transducer. Tiejian Xia (Hangzhou Applied Acoustics Research Institute, 401\# Fuyang, Zhejiang, 311400 Fuyang, China, xiatiejian @ 263.net), Yuebing Wang (Hangzhou Applied Acoustics Research Institute, 96 Huaxing Road, 310012 Hangzhou, China, wang_yuebing@tom .com), Lisheng Zhou (Rensselaer Polytechnic Institute, Greene Building, School of Architecture, 110 8th Street, Troy, NY 12180, USA, 1szhou@163.com), Qiang Liu (Hangzhou Applied Acoustics Research Institute, 401\# Fuyang, Zhejiang, 311400 Fuyang, China, 715liuqiang@ 163 .com)
The BEM software is implemented in the prediction of the acoustic field produced by a cylindrical transducer, the transducer consists of 1200 PZT pillars which are fixed on the surface of a 156-mm-diameter and 120-mmheight cylinder. The acoustic field around the transducer is measured at the frequency $120 \mathrm{kHz}$ using an optical vibrometer, the measured data are fed into the BEM software as the boundary condition, and the directivities of the transducer are forecasted in the vertical and horizontal planes. To verify the calculation, the acoustic field in the far field of the transducer is measured using a hydrophone, and good agreements between the prediction and the measurement convince that the BEM software can be used to predict acoustic fields of cylindrical transducers through distributions of acoustic fields measured in their near fields. 


\title{
Session 3aUWc
}

\section{Underwater Acoustics, Signal Processing in Acoustics, and ECUA: Acoustic Vector Fields and Sensor Processing II}

\author{
Kevin B. Smith, Cochair \\ Naval Postgraduate School/Naval Undersea Warfare Center, Code PH/Sk, Department of Physics, Monterey, CA 93943, USA \\ Jean-Pierre Hermand, Cochair \\ Université libre de Bruxelles (U.L.B.) - Environmental hydroacoustics lab, av. Franklin D. Roosevelt 50, CP 194/5, Bruxelles, \\ 1050, Belgium
}

Invited Papers

10:40

3aUWc1. Acoustic vector sensors and vector/tensor processing in ocean acoustics research. Gerald D'Spain (Marine Physical Lab, Scripps Institution of Oceanography, 291 Rosecrans St., San Diego, CA 92106, USA, gld@mpl.ucsd.edu)

Several passive sensor systems that simultaneously measure acoustic pressure and the three components of acoustic particle motion have been designed and deployed in seagoing experiments by the Marine Physical Lab over the past two decades. Descriptions of these sensor systems and results from applying various signal/array processing methods with these data are presented in this talk. Although vector sensors do not provide any additional information on the sound field than that obtained by a suitable number of properly placed hydrophones, they do provide estimates of the sound field's directional properties from a very compact package. Applications where available space is severely limited include autonomous underwater vehicles (AUV). Recent results from acoustic vector sensors installed in a flying wing underwater glider and inside a prop-driven AUV to quietly measure sounds in the ocean illustrate the benefits, and drawbacks, of the use of vector sensors on autonomous platforms. The extension of the signal/array processing approaches to tensor sensors indicates the promise of additional gain, if the sensor design challenges can be overcome. [Work supported by the Office of Naval Research].

\section{1:00}

3aUWc2. Robust Adaptive Vector Sensor Processing. Andrew J. Poulsen (Massachusetts Institute of Technology, 77 Massachusetts Avenue, Cambridge, MA 02139, USA, poulsen@mit.edu), Arthur B. Baggeroer (MIT, Department of Mathematics, 77 Massachusetts Avenue, Cambridge, MA 02139, USA, abb@boreas.mit.edu), Jonathan Paul Kitchens (MIT Lincoln Laboratory, 244 Wood Street, Lexington, MA 02420, USA, jonpaul@ mit.edu), Jennifer A. Watson (MIT Lincoln Laboratory, 244 Wood Street, Lexington, MA 02420, USA, watson@11.mit.edu)

A sizable literature exists on the theory of processing signals for many vector sensor array applications. In practice, however, mismatch and several noise processes can pose significant problems. If adaptive beamforming is also used, the "snapshot" issue potentially increases by a factor of four since each element in a vector sensor array consists of a scalar hydrophone and up to three spatially orthogonal particle motion sensors. Both of these sensor types have very different response and noise characteristics. Particle motion sensors are more sensitive to non-acoustic, motion-induced noise than hydrophones. In towed line array configuration, those sensors orthogonal to the direction of motion are exposed to higher intensities of flow noise at low frequencies than those coincident to the array axis. Similarly, different dipole sensors may be exposed to varying degrees of rotational mismatch. Sensors may also rest on the seafloor, creating asymmetries. Physical constraints also relate pressure and particle velocity measurements via conservation of momentum and mass equations. We examine adaptive processing methods customized to the unique characteristics of vector sensors. Array gain and other performance metrics in the presence of system mismatch and uncertainties is presented using common ocean noise models, including directional jamming, isotropic and surface noise.

\section{$11: 20$}

3aUWc3. Using acoustic streamlines to visualize energy flow across boundaries. David M.F. Chapman (Scientific Consultant, 8 Lakeview Avenue, Dartmouth, NS B3A 3S7, Canada, dave.chapman@ns.sympatico.ca)

For spherical waves that radiate from a point source in a homogeneous fluid and propagate across a plane boundary into a dissimilar homogeneous fluid, the acoustic field may differ significantly from the geometric acoustics approximation if either the source or receiver is near the interface (in terms of acoustic wavelengths) or if the minimum-phase path is near the critical angle. Cross-boundary energy flow from a continuous-wave point source is visualized by tracing acoustic streamlines: those curves whose tangent everywhere is the average acoustic intensity vector. It is seen that the acoustic energy flow is not always in line with the "Snell's law" or minimum-phase ray path. Also, acoustic energy streamlines do not display unusual behaviour in the vicinity of the critical angle. Finally, it is shown that there exists a law of refraction of acoustic energy streamlines across density discontinuities, analogous to Snell's law of refraction of ray paths across sound speed discontinuities. Examples include water-to-seabed transmission and water-to-air transmission. [Work supported in part by ONR Code 32] 
3aUWc4. Real time sound field visualization in the near field, far field and at absorbing surfaces. Hans-Elias De Bree (Microflown Technologies Inc., PO BOX 300,6900 AH Zevenaar, Netherlands, debree@microflown.com), Emiel Tijs (Microflown Technologies Inc., PO BOX 300, 6900 AH Zevenaar, Netherlands, tijs@ microflown.com), Tom Basten (TNO Science and Industry, Stieltjesweg 1, 2600 AD Delft, Netherlands, tom.basten@tno.nl)

The Microflown is a small sensor that is capable of measuring the acoustic particle velocity. In combination with a small pressure microphone it is possible to measure the complete sound field. If the sound field close to an acoustic material is measured it make sense to measure the particle velocity normal to the surface and the sound pressure. With this, the acoustic impedance and absorption can be measured in real time, and with a high spatial resolution. If the sound field of a sound radiating object is measured, the sound field can be obtained directly. Three applications include: direct acoustic camera, that is the real time display of the sound field (velocity, pressure, intensity). Holography that is the pressure, velocity reconstruction on the surface based on the measured sound field (and other places). And cabin interior noise mapping, that is the visualization of the interior noise perceived on a certain location. With three dimensional sound probes (sound pressure and particle velocity in three dimensions), sources in the free field can be found for both bearings as distance. Most recent developments are presented.

\section{2:00}

3aUWc5. Measurement of total sound energy density in enclosures at low frequencies. Finn Jacobsen (Acoustic Technology Department, Technical University of Denmark, Ørsted Plads, B352, DK-2800 Lyngby, Denmark, fja@oersted.dtu.dk)

Many acoustic measurements rely on determining the total sound energy in an enclosure; and this quantity is usually estimated by measuring the mean square pressure at a number of discrete positions. Almost 30 years ago it was shown theoretically that the normalised spatial variance of the total sound energy density (potential and kinetic) is one third of the normalised spatial variance of the potential energy density (the mean square pressure) in a reverberant sound field above the Schroeder frequency. About ten years later this prediction was confirmed experimentally. However, until recently measurement of the total sound energy density (in air) has required an elaborate arrangement based on finite different approximations using at least four matched pressure microphones; therefore the method has never come into use. However, with the advent of a three-dimensional particle velocity transducer it has become somewhat easier to measure total rather than only potential energy density in a sound field. This paper examines the spatial uniformity of potential, kinetic and total sound energy density in enclosures theoretically and experimentally with particular emphasis on the frequency range below the Schroeder frequency.

\section{Contributed Papers}

12:20

3aUWc6. On the usefulness of waterborne measurement of particle velocity in geoacoustic inversion. Jean-Pierre Hermand (Université libre de Bruxelles (U.L.B.) - Environmental hydroacoustics lab, av. Franklin D. Roosevelt 50, CP 194/5, 1050 Bruxelles, Belgium, jhermand@ulb ac.be), Kevin B. Smith (Naval Postgraduate School/Naval Undersea Warfare Center, Code PH/Sk, Department of Physics, Monterey, CA 93943 , USA, kbsmith@nps.edu)

Recent advances in sensor design have led to the development of receiving systems whose elements are vector sensors, i.e., sensors that simultaneously measure the acoustic pressure and fluid motion due to the propagation of acoustic energy at the sensor location. As such, arrays of vector sensors can provide more information about the sound field than arrays made of traditional hydrophones, and thus are attractive for various applications including the inversion for environmental properties. The fundamental question addressed by this paper is: does the use of acoustic vector data versus pressure-only data improve the results of an inversion scheme based on waterborne observations and matched field processing? To our knowledge no experimental data are available yet to allow a detailed comparison of performance between standard (pressure-only) arrays and vector sensor arrays for environmental inversions. For a preliminary study we will simulate the inversion of vector sensor data in the South Elba environment for which pressure-only inversion results and ground truth data are available following the Yellow Shark'94 and Blue Planet'07 experiments. Broadband signals received on fully-populated and sparse arrays will be considered.

\section{2:40}

3aUWc7. Characterization of the Near Scattered Acoustic Vector Field. Robert Barton (Naval Undersea Warfare Center, Code 1522 bldg1320, 1176 Howell st, Newport, RI 02841, USA, bartonrj@npt.nuwc.navy.mil), Kevin B. Smith (Naval Postgraduate School/Naval Undersea Warfare Center, Code PH/Sk, Department of Physics, Monterey, CA 93943, USA, kbsmith @ nps.edu)

In this study, we investigate the properties of the scattered acoustic vector fields generated by simple geometric objects, including the infinite rigid plate, disk, and sphere. Analytical solutions are derived from acoustic target strength scattering models in the near field region. Of particular interest is the understanding of the characteristics of energy flow of the scattered acoustic vector field in the near to far-field transition region. We utilize the time and space separable instantaneous active and reactive acoustic intensity to investigate the relative phase properties of the scattered field. Numerical results are presented for the near region scattered acoustic vector field of simple objects in both two and three dimensions. 


\title{
Session 3aUWd
}

\section{Underwater Acoustics and ECUA: Geoacoustic Sediment Modeling II}

\author{
Nicholas P. Chotiros, Cochair \\ Applied Research Laboratories, University of Texas, PO Box 8029, Austin, TX 78713-8029, USA \\ Jean-Pierre Sessarego, Cochair \\ Laboratory for Mechanics and Acoustics CNRS, 31 chemin Joseph Aiguier, Marseille, 13009, France
}

\section{Invited Papers}

\section{1:20}

3aUWd1. The Viscous-Grain-Shearing (VGS) theory of wave propagation in marine sediments. Michael Buckingham (Marine Physical Lab, Scripps Institution of Oceanography, UCSD, 9500 Gilman Dr. M/C 0238, La Jolla, CA 92093-0238, USA, mjb@mpl .ucsd.edu)

The grain-shearing (GS) theory of wave propagation in a marine sediment is based upon the idea that, as grains slide against one another, the dissipation becomes progressively stronger, a phenomenon known as strain hardening. The GS theory predicts a sound speed showing weak, logarithmic dispersion and an attenuation that scales linearly with frequency. At frequencies above about $10 \mathrm{kHz}$, such behavior matches the sound speed and attenuation data obtained during the SAX99 experiment in the Gulf of Mexico. But at lower frequencies, the measured dispersion curves show a lower sound speed and higher attenuation than predicted by the GS theory. A generalized version of the GS theory, designated the VGS theory, takes account of the effective viscosity of the molecularly thin layer of pore fluid between contiguous grains, which limits the degree of strain hardening that can occur during sliding. The VGS dispersion expressions are the same as those of the GS theory, except for the appearance of a simple algebraic function that has an effect only at low frequencies, below $10 \mathrm{kHz}$. The resultant VGS curves match the sound speed and attenuation measurements from SAX99 over the measurement frequency band from 1 to $400 \mathrm{kHz}$. (Research supported by ONR.)

\section{1:40}

3aUWd2. Analysis of acoustical transmission and reflection from a sandy stratified rough sediment using the grain shearing model. Laurent Guillon (Ecole Navale/Irenav, BP 600, 29240 Brest Armées, France, guillon@ecole-navale.fr), Vladislav Aleshin (LPEC/UMR 6087/CNRS/Université du Maine, Avenue Olivier Messiaen, 72085 Le Mans Cedex 09, France, aleshinv@mail.ru)

A number of applications, such as geoacoustic inversion or object detection, require an appropriate theoretical consideration of the sound reflection and transmission problem. The analysis we present here includes three essential aspects: account for a constitutive behavior of a saturated unconsolidated granular material, presence of vertical gradients in material properties, and roughness of the sediment surface. Accepting the Buckingham grain shearing model (see e.g. JASA, vol. 117, pp. 137-152) for the stress-strain relationship in the sediment, the gravity-induced variations of the longitudinal and shear rigidity can be described. The corresponding numerical solution for the plane interface is presented and the influence of infinite rigidity gradients at the surface is discussed. Another important contribution is provided by the interface roughness that considerably improves the penetration conditions. This problem is solved using a statistical treatment of a number of roughness realizations with a given spatial spectrum. The developed numerical tool enables to study the influence of all these factors and estimate the penetrated energy for different acoustical frequencies, roughness parameters, and grain sizes (This work was founded by DGA and Thales Underwater Systems).

\section{2:00}

3aUWd3. Bistatic scattering in sediments: comparison of model and scaled tank experiments at 238 kHz. Russell Howey (University of Bath, Department of Physics, Claverton Down, BA2 7AY Bath, UK, rph23@ bath.ac.uk), Philippe Blondel (University of Bath, Department of Physics, Claverton Down, BA2 7AY Bath, UK, pyspb@bath.ac.uk)

Multiple-aspect scattering is increasingly used to investigate seabeds and objects, buried or not. However, high-frequency scattering processes on/in sediments need to be better understood, particularly when the structure and/or composition of these sediments is not fully homogeneous. Scaled tank experiments were conducted using the University of Bath facilities. A narrow acoustic beam $\left(10^{\circ}\right.$ beamwidth) was generated by a transducer resonant at $238 \mathrm{kHz}$, imaging a silt seabed at $45^{\circ}$. Scattering angles varied between $\sim 16^{\circ}$ and $\sim 70^{\circ}$ (50 distinct values); bistatic angles varied $40^{\circ}$ either side of in-plane with a $2.5^{\circ}$ step (33 distinct values). Bottom returns were picked through two methods (automatic and manual) and converted into scattering strengths, accounting for the size of the scattering patch. This large dataset has been compared with the model of Jackson and Williams for bistatic scattering, intended for 10-100 kHz but successfully tested at $240 \mathrm{kHz}$ by other workers. Recursive fitting of model parameters to the experimental values shows the influence of sediment variations (backed by microscopic measurements of actual grain size distributions) and the importance of even small tilts in the surface of the sediments. These results can be used to test the validity of extending this scattering model to higher frequencies. 
3aUWd4. Dispersion and attenuation due to scattering from heterogeneities in the frame bulk and shear moduli of sand sediments. Brian T. Hefner (Applied Physics Laboratory, University of Washington, 1013 NE 40th St, Seattle, WA 98105, USA, hefner@apl.washington.edu), Darrell R. Jackson (Applied Physics Laboratory, University of Washington, 1013 NE 40th Street, Seattle, WA 98105, USA, drj@apl.washington.edu), Joseph Calantoni (Naval Research Laboratory, Marine Geosciences Division, Code 7440.3, Building 1005, MS 22217, USA, joec@nrlssc.navy.mil)

Previously, we presented the results of applying perturbation theory to the problem of fast compressional wave propagation through a Biot medium with heterogeneities in the frame bulk modulus (B. T. Hefner et al., J. Acoust. Soc. Am. 119, 3447 (2006)). It was found that the heterogeneities scattered energy into both the slow and fast compressional waves, thus increasing the attenuation of the fast compressional wave. This theory has since been generalized to account for heterogeneities in both the frame bulk and shear moduli. For the fast compressional wave, energy is now scattered into the shear wave as well as the fast and slow compressional waves, further increasing the attenuation of the coherent field. While shear wave propagation is unaffected by variations in the frame bulk modulus, scattering does occur when there are heterogeneities in the shear modulus. Energy in the shear wave is scattered into both shear and compressional waves as well. The generalized theory depends on the autocorrelation functions of both the shear and bulk moduli variations as well as the cross-correlation function between the moduli. Efforts are underway to estimate these statistics in simple random packings of spherical grains using discrete-element modeling. [Work supported by ONR.]

\section{2:40-2:00 Lunch Break}

\section{Invited Papers}

\section{2:00}

3aUWd5. Data-model comparisons for broadband sound speed and attenuation in sandy seabed. Ji-Xun Zhou (School of Mech. Eng., Georgia Institute of Technology, 771 Ferst Drive, Atlanta, GA 30332-0405, USA, jixun.zhou@me.gatech.edu), Xue-Zhen Zhang (School of Mech. Eng., Georgia Institute of Technology, 771 Ferst Drive, Atlanta, GA 30332-0405, USA, xuezhen.zhang@me .gatech.edu)

Sound speed and attenuation in sandy seabed were analyzed and reviewed from low-frequency (LF) acoustic measurements, conducted at 18 locations in different coastal zones around the world. [Zhou and Zhang, JASA 117(4), 2494 (2005) and 119 (5), 3447 (2006)]. The resultant sound speed and attenuation in a frequency range of 50-2000 Hz in sandy bottoms can be described equally well by the Biot-Stoll poro-elastic model, the Chotiros BICSQS model [JASA 116(4), 2011-2022 (2004)] or the Buckingham VGS model [JASA 122(3), 1486-1501(2007)]. The geophysical parameters were inverted from the best match between the LF data and each of these three geo-acoustic models. The values of the inverted geophysical parameters were reasonable based on both theoretical considerations and published experimental measurements. A combination of the LF field-inverted data with data from the SAX99 experiment and other recent high-frequency measurements offers a broadband reference data set of sound velocity and attenuation for sandy bottoms in shallow water over a frequency range of $50-400,000 \mathrm{~Hz}$. The data-model comparisons show that, with one set of adjustable input parameters, all three geo-acoustic models may perfectly match either the broadband velocity dispersion or broadband attenuation, but not both. These models generally either underestimate dispersion or overestimate LF attenuation. [Work supported by ONR.]

3aUWd6. Role of heterogeneity for sound speed and attenuation in unconsolidated ocean sediments. Jim G. Berryman (Lawrence Berkeley National Laboratory, 1 Cyclotron Road MS90R1116, Berkeley, CA 94720, USA, JGBerryman@LBL.GOV), Steven R. Pride (Lawrence Berkeley National Laboratory, 1 Cyclotron Road MS90R1116, Berkeley, CA 94720, USA, SRPride@LBL.GOV)

Although ocean sediments may have many types and degrees of heterogeneity, it has been determined that two-component models are tractable for poroelastic, thermoelastic, and thermoporoelastic studies. We have found in particular that a substantial part of the increased sound wave attenuation observed in both ocean sediments and laboratory glass-bead experiments above $50 \mathrm{kHz}$ can be explained by such a two-component poroelastic model. Furthermore, the inclusion of thermal expansion and entropy effects into a generalized binary thermoporoelastic model provides an additional attenuation enhancement (though not as large --- by itself --- as the poroelastic enhancement) of a mathematically very similar type that is also relatively easy to model and understand. The paper will present this extended model as well as comparisons to available data.

\section{2:40}

3aUWd7. Frequency and grain size dependence of longitudinal wave velocity in granular marine sediments. Masao Kimura (Tokai Univ., 3-20-1 Orido, Shimizu-ku, 424-8610 Shizuoka, Japan, mkimura@ @cc.u-tokai.ac.jp)

Frequency dependence of longitudinal wave velocity in granular marine sediments is important in underwater acoustics. Also, the grain size dependence is significant for the identification of sediments. In this study, the measurements of the velocity of three kinds of sands with different grain sizes are carried out in the frequency ranges from approximately $5 \mathrm{kHz}$ to $500 \mathrm{kHz}$ in the laboratory. The velocity at a low frequency is measured by using an acoustic tube method. Time-of-flight method is used for the measurements of the velocity at higher frequencies. These measured results are compared with the calculated results by using the Biot-Stoll model and a modified gap stiffness model incorporated into the Biot model (BIMGS model). It is shown that the frequency and grain size dependence of velocity can be explained by using the BIMGS model. 
3aUWd8. Biot-Stoll with squirt flow and shear (BICSQS) model: high-frequency correction. Nicholas P. Chotiros (Applied Research Laboratories, University of Texas, PO Box 8029, Austin, TX 78713-8029, USA, chotiros@arlut.utexas.edu)

The modeling of the grain to grain shear stiffness within the larger Biot-Stoll plus contact squirt flow and shear (BICSQS) model [N. P. Chotiros and M. J. Isakson, J. Acoust. Soc. Am. 116(4), 2011-2022, 2004] assumes Poiseuille flow, which must become invalid at high-frequencies. Specifically, the viscous shear drag term will be unphysically large. A correction is introduced based on Biot's solution for oscillatory flow between two parallel plane boundaries [M. A. Biot, J. Acoust. Soc. Am. 28, 179-191, 1956]. Although the original solution was derived for oscillatory fluid flow relative to stationary parallel plane boundaries, it can be readily adapted for oscillatory motion of the boundaries in opposite directions relative to the fluid, as in shear drag at the grain to grain contact. This correction extends the frequency range of the BICSQS model. Theoretical results, with and without the correction, will be compared. Work supported by the Office of Naval Research, Ocean Acoustics.

\title{
Contributed Paper
}

\section{$3: 20$}

3aUWd9. Use of dual methods to infer methane bubble populations in gassy sediment: Inversion of propagation data. Gary B. Robb (National Oceanography Centre, University of Southampton Waterfront Campus, European Way, SO14 3ZH Southampton, UK, gbor199@noc.soton.ac .uk), Timothy G. Leighton (Institute of Sound and Vibration, Univ. of Southampton, University Road, Highfield, SO17 1BJ Southampton, UK, T.G.Leighton@soton.ac.uk), Agni Mantouka (Institute of Sound and Vibration, Univ. of Southampton, University Road, Highfield, SO17 1BJ Southampton, UK, am2@isvr.soton.ac.uk), Angus I. Best (National Oceanography Centre, University of Southampton Waterfront Campus, European Way, SO14 3ZH Southampton, UK, aib@noc.soton.ac.uk), Justin K. Dix (National Oceanography Centre, University of Southampton Waterfront Campus, European Way, SO14 3ZH Southampton, UK, jkd@ noc .soton.ac.uk), Victor F. Humphrey (Institute ofSound and Vibration, Univ. of Southampton, University Road, Highfield, SO17 1BJ Southampton, UK, vh@isvr.soton.ac.uk), Zygmunt Klusek (Institute of Oceanography, Polish
Academy of Science, P.O. Box 148 Sopot, Poland, klusek@iopan .gda.pl), Paul R. White (Institute of Sound and Vibration, Univ. of Southampton, University Road, Highfield, SO17 1BJ Southampton, UK, prw@isvr.soton.ac.uk)

The inversion of the acoustic properties of gassy sediments presents the optimum manner of determining the in situ distribution of sediment-based methane bubbles. An in situ device that measures both compressional wave attenuations and combination-frequency components in gassy sediment lying within $2 \mathrm{~m}$ of the seabed has been developed at the University of Southampton. This device was deployed at an inter-tidal site along the South coast of England. Compressional wave attenuations were measured from 10 to $100 \mathrm{kHz}$ though the analysis of propagation signals transmitted from a variety of sources to a buried co-linear hydrophone array, with propagation distances spanning 0.5 to $2 \mathrm{~m}$. Measured attenuations were inverted to infer in situ bubble size distributions using both established and new acoustic models for gassy sediment. The analysis and results of the combinationfrequency component are described in a companion paper.

\section{Session 3pAA}

\section{Architectural Acoustics: Architectural Acoustics Potpourri I}

\author{
David T. Bradley, Cochair \\ 124 Raymond Avenue, Poughkeepsie, NY, 12604 \\ Andrea Farnetani, Cochair \\ University of Ferrara, Via Saragat 1, 44100 Ferrara, Italy
}

\section{Contributed Papers}

\section{2:00}

3pAA1. Shape optimization of polygonal rooms for a correct modal distribution at low frequencies based on psychoacoustic criterion. Sergio Floody (Universidad Tecnologica de Chile INACAP, Brown Norte 290, Nunoa, 7790569 Santiago, Chile, sfloody@utc.cl), Rodolfo Venegas (University of Salford, Acoustics Research Centre, Newton Building, M5 4WT Salford, UK, R.G.VenegasCastillo@pgr.salford.ac.uk)

Resonances in small rooms may lead to inadequate frequency responses. In rooms, where the exigencies on the listening conditions are important, these resonances may cause non wanted coloration effects, which implies a non desirable sound quality. By choosing the right shape and dimensions it is possible to reduce the audible effects of these resonances. The presented methodology aims to determine the shape and size of small and medium polygonal-shaped rooms based on the finite element method for modeling the physical acoustic behavior of the room; a neural network for loudness estimation and genetic algorithm for estimating the optimal dimensions. A comparison with previous techniques used to choose the dimension of rectangular room is also presented.

2:20

3pAA2. The reflected sound field by curved surfaces. Martijn Vercammen (Peutz, De Grippen 1124, 6605 TA Wijchen, Netherlands, m.vercammen@mook.peutz.nl)

Many spaces have curved walls or ceilings. With improved building technology and new fashions in architecture (blobs) there is an increasing number of problems due to the acoustic reflections by these surfaces. Sound reflected by concave surfaces will concentrate in a narrow area. In practical applications of room acoustics these curved surfaces will be calculated with mirror imaging or ray tracing programs, in which the structure is modeled by flat segments. Alternative is a geometrical approach. Both methods do 
not correspond to reality. The only valid calculation method is the calculation from a wave extrapolation method. It is shown that a theoretical correct solution of the sound field by curved surfaces is possible. A fairly simple expression for the sound pressure in the focal point is found and a more complicated description of the reflected sound field by small curved surfaces is presented. With these results the sound field in field applications can be calculated.

\section{2:40}

3pAA3. Calculation of temporal evolution of sound pressure levels in rooms, based on diffuse reflection. René Gamba (Gamba Acoustique, 2 rue de la découverte, BP 163, 31676 Labege Cedex, France, rene.gamba@acoustique-gamba.fr), Guillaume Cazard (Gamba Acoustique, 2 rue de la découverte, BP 163, 31676 Labege Cedex, France, guillaume.cazard@acoustique-gamba.fr), Claude Senat (Gamba Acoustique, 2 rue de la découverte, BP 163, 31676 Labege Cedex, France, claude .senat@acoustique-gamba.fr)

This paper describes a model which enables the temporal evolution of sound pressure levels in rooms to be calculated. It is built on the assumption that sound waves are totally scattered when reflected by the walls. This model prescribes the sampling of all the surfaces of the room and defines a process of time discretization, process which enables the temporal evolution of energetic exchanges between each sample to be known. Ultimately, the model allows the sound pressure level to be calculated in every point of the room for each time sample $\Delta \mathrm{t}$ (echograms). Thanks to the echograms, some useful criterias for room acoustic studies can be evaluated : reverberation time, EDT, D50, C80,... Measurements and calculations have been carried out for different kinds of rooms. We will describe them in the second part of this paper.

\section{3:00}

3pAA4. Sound absorption with fibre-free sintered aluminium in combination with thermally activated concrete slabs. Wouter Rottiers (Sonogamma, P.O.Box 49, Heverlee Ambassade, 3001 Leuven, Belgium, info@sonogamma.com)

Due to increasing ecological awareness and growing requirements of human well-being in working environments, contemporary interior design requires sustainable solutions. Consequently, cooling ceilings are an efficient alternative to conventional air conditioning systems with high energy demands. In order to maintain its thermal efficiency however, the ceiling surface must remain uncovered as much as possible. Conventional perforated or grooved acoustical materials allow for sound absorption only in combination with mineral wool or foam, thus presenting a thermal isolating barrier: they obviously decrease the performance of a thermal ceiling. Porous panels made of pure sintered aluminium offer a conductivity up to 96 $\mathrm{kcl} / \mathrm{mhC}$ with a thermal radiation of $18.4 \mathrm{~W} / \mathrm{m}^{2} \mathrm{~K}$, combined with a broadband sound absorption up to $\alpha \mathrm{w}=0.7$ without the use of any additional thermally isolating material. The panels meet the environmental, hygienic and fire safety requirements: they are fibre-free, non-combustible (Euroclass A1), rust-, moisture- and chlorine-proof and can be recycled. The unvisible porosity does not give away the outstanding acoustical qualities. Available in several designs and any colour, the sheets are optically identical to a plain, matt aluminium panel. Several possible configurations are discussed.

\section{3:20}

3pAA5. Sound reduction of open noise screens. Jan Hardlooper (Cauberg-Huygen Consulting Engineers, Postbus 9222, 3007 AE Rotterdam, Netherlands, j.hardlooper@chri.nl)

Open noise screens consist of vertical absorbing screens with a depth of 1 meter, placed perpendicular to a building with a mutual distance of 1 meter. The screens are placed at a distance of approximately 1,5 meter. Open noise screens combine a sound reduction of $16 \mathrm{~dB}$ with a very open character for ventilation and daylight admittance. Measurements are carried out on a scale model (1:40) in a laboratory and in situ on a real size mock-up, built in a 40-ft container. The results of the mock-up are comparable to the laboratory results. Frequency analysis shows the acoustical principles of the screens and the influence of several parameters. A calculation model is developed tot predict the results for other configurations. The principle of open noise screens is successfully applied for 200 new dwellings in Amsterdam. Suggestions are given for applying open noise screens near highways and railroads, instead of traditional closed screens. 


\title{
Session 3pAB
}

\section{Animal Bioacoustics, Psychological and Physiological Acoustics, and ECUA: Auditory Brainstem Response and Behavior Correlation I}

\author{
Elizabeth Brittan-Powell, Cochair \\ Dept of Psychology, University of Maryland, College Park, MD 20742, USA \\ Alexander Y. Supin, Cochair \\ Institute of Ecology and Evolution, 33 Leninsky Prospect, Moscow, 119071, Russian Federation
}

Invited Papers

2:00

\begin{abstract}
3pAB1. Auditory brainstem responses in birds: How well do they compare to behavioral techniques for assessing hearing? Elizabeth Brittan-Powell (Dept of Psychology, University of Maryland, College Park, MD 20742, USA, bbrittanpowell@psyc .umd.edu), Bernard Lohr (Department of Biological Sciences, Northern Kentucky University, SC 204D, Nunn Drive, Highland Heights, AK 41099, USA, lohrb1@nku.edu), Isabelle Noirot (Dpt of Science/Oceanography, University of Liege, Bat B6c, 4000 SartTilman, B-4000 Liege, Belgium, isa_noirot@hotmail.com), Robert Dooling (Dept of Psychology, University of Maryland, College Park, MD 20742, USA, dooling@psyc.umd.edu)
\end{abstract}

Behavioral methods are the gold standard for assessing hearing sensitivity in birds. In many cases, however, behavioral techniques are simply impractical. The non-invasive auditory brainstem response (ABR) represents perhaps the next best approach, and researchers are increasingly turning to the ABR to obtain estimates of hearing sensitivity, auditory system function and development. Our lab has evaluated hearing sensitivity in over 11 species of birds using the ABR, and for six of these species, we have also obtained auditory thresholds by behavioral conditioning methods. These data, along with other results from the literature on bird hearing, now allow general conclusions about the use of ABR as a tool for measuring auditory sensitivity in birds. Waveform morphology is highly conserved across orders of birds. Regardless of how threshold is defined, the ABR audiogram reliably reflects the frequency range of hearing and the shape of the behavioral audiogram. However, ABR thresholds are higher than behavioral thresholds by 20-30 dB but with some variation across species. Aside from audiograms based on simple detection thresholds for tone bursts, ABR methods in birds have also been useful in assessing auditory development, hearing impairment, recovery of function with hair cell regeneration, masked thresholds and aspects of spectral and temporal processing.

$$
\text { 2:20 }
$$

3pAB2. The Auditory Brainstem Response (ABR) Across Mammalian Species. Robert Burkard (University at Buffalo, Department of Rehabilitation Science, 511 Kimball Tower, Buffalo, NY 14214, USA, rfb@buffalo.edu)

The auditory brainstem response (ABR) has been reported in a variety of mammalian species. I have published ABR studies in mammalian species including humans, gerbils, cats, rats, bats, and mice. In many cases, the non-human work was designed to extend observations made in humans. I will discuss data that addresses the variability in the slope of the ABR latency/intensity function across animal species. I will compare ABRs in humans and in gerbils to stimulus manipulations such as click level, click rate, noiseburst risetime and level of masking noise. I will discuss the effects of high stimulus rates across age in the kitten/cat, and compare this to reports in humans from the literature. I will end by discussing my views of the advantages as well as the challenges of using animal models when interested in human normative or pathologic processes.

\section{Contributed Paper}

\section{2:40}

3pAB3. Arbitrary evoked potentials: using AEPs to measure hearing in fishes. David Mann (University of South Florida, College of Marine Science, 140 7th Ave. S., St. Petersburg, FL 33701, USA, dmann@marine .usf.edu), Randy Hill (University of South Florida, College of Marine Science, 140 7th Ave. S., St. Petersburg, FL 33701, USA, randyjhill@yahoo .com)

Auditory evoked potential (AEP) techniques have become commonly used to measure hearing of a wide variety of animals. However, there is no standard way of defining a threshold from auditory evoked potential data. There are two arbitrary factors affecting the precision of AEP data: the number of sweeps averaged together to detect the AEP, and the method of calculating a threshold from the AEP data. While these arbitrary factors do not invalidate comparative studies where these variables are standardized, different laboratories use different techniques. We describe sources of variation in estimating thresholds using evoked potential techniques using the goldfish as an example. There was little variation in AEP thresholds due to variation in electrode placement or in the method of arbitrarily assigning an AEP threshold. The largest variation was due to differences in the number of sweeps averaged. Under controlled conditions using the same goldfish in the same test tank with the same number of sweeps averaged, AEP's better predicted behavioral thresholds at high frequencies than low frequencies. Since averaging reduces uncorrelated background noise (neural and electrical), it should theoretically be possible to obtain AEP measurements below behavioral thresholds with enough sweeps averaged together. 


\title{
Invited Papers
}

3:00

3pAB4. False killer whale hearing adjustment during echolocation measured with evoked potentials. Paul E. Nachtigall (University of Hawaii, Hawaii Institute of Marine Biology, P.O. Box 1106, Kailua, HI 96734, USA, nachtiga@ hawaii.edu), Alexander Y. Supin (Institute of Ecology and Evolution, 33 Leninsky Prospect, 119071 Moscow, Russian Federation, alex_supin@mail.ru)

While much has been previously learned about the echolocation performance and characteristics of the outgoing signals of echolocating dolphins and small whales, we have measured hearing using evoked potentials during echolocation. We have found that: (1) the whale may hear her loud outgoing clicks and much quieter returning echoes at comparable levels, (2) the whale has protective mechanisms and hears her outgoing signals at a level about $40 \mathrm{~dB}$ lower than similar signals presented directly in front of her, (3) when echo return levels are lowered either by making the targets smaller or by placing the targets farther away - without changing the levels of her outgoing signals, the hearing of those echoes remains at almost the same level, (4) if targets are made much smaller and harder to echolocate, the animal will increase what she hears of her outgoing signal - as if to heighten overall hearing sensitivity to keep the echo level hearable, and (5) the animal has an active 'automatic gain control' mechanism in her hearing based on both forward masking that balances outgoing pulse intensity and time between pulse and echo and active hearing control. Overall, hearing during echolocation appears to be an actively changing process.

$3: 20$

3pAB5. Temporal resolution in the hearing system and auditory evoked potentials. Kristian Beedholm (Institute of Biology, University of Southern Denmark, Campusvej 55, 5230 Odense M, Denmark, beedholm@mail.dk), Lee A. Miller (Institute of Biology, University of Southern Denmark, Campusvej 55, 5230 Odense M, Denmark, lee@biology.sdu.dk)

A popular type of investigation with auditory evoked potentials (AEP) consists of mapping the dependency of the envelope following response to the AM frequency. This results in what is called the modulation rate transfer function (MRTF). The physiological interpretation of the MRTF is not straight forward, but is often used as a measure of the ability of the auditory system to encode temporal changes. It is, however, shown here that the MRTF must depend on the waveform of the click-evoked AEP (ceAEP), which does not relate directly to temporal resolution. The theoretical relationship between the spectrum of the ceAEP and MRTF is such that the MRTF should be identical to the ceAEP if a 1/f weighting is applied to the ceAEP. Deviations from this relationship indeed reflect temporal resolution capabilities. We measured this in a harbour porpoise. Our devised stimulus was a sequence of $0.5 \mathrm{~ms}$ Hann weighted $130 \mathrm{kHz}$ tone pips presented at an increasing rate (chirped) over a time span of $32 \mathrm{~ms}$. The results reveal that the system's responsiveness declines roughly exponentially as a function of click rate with a rate constant of about $-0.7 \mathrm{kHz}$ and appears more rate limited than implied by traditional MRTF.

WEDNESDAY AFTERNOON, 2 JULY 2008

AMPHI MAILLOT, 1:40 TO 3:40 P.M.

\section{Session 3pMUa}

\section{Musical Acoustics: Bowed and Keyboard Stringed Instruments I}

\author{
Knut Guettler, Cochair \\ Norwegian Academy of Music, P.O.Box 5190 Majorstuen, Oslo, 0302, Norway \\ Claudia Fritz, Cochair \\ University of Cambridge, Music Faculty, West Rd, Cambridge, CB3 9DP, UK
}

\section{Contributed Papers}

\begin{abstract}
$1: 40$
3pMUa1. On the dynamics of the clavichord. Christophe D’Alessandro (LIMSI-CNRS, B.P. 133, 91403 Orsay, France, cda@limsi .fr)

The clavichord is generally considered as the most sensitive and subtle among keyboard instruments. The player/instrument interaction is very direct: the mechanism is reduced to as simple lever, allowing for a direct contact between the finger and string through the key. Key velocity, two string-tangent contact signals, radiated acoustic signal have been synchronously measured for about 10 dynamic nuances and all the notes of four instruments (a 51 notes unfretted instrument, after a German XVIIIth century model, a 51 notes fretted instrument after Hubert (1754), a 45 notes fretted instrument, after a German XVIIth century model, a 37 notes fretted instrument after a medieval model). The instruments can be portrayed in terms of dynamic range, tonal/spectral colour and sound decay time. As for
\end{abstract}

the dynamics, there is some evidence for a linear relationship between sound pressure level and the velocity of the tangent; and an almost constant spectral richness independently of loudness (in contrast with e.g. the piano). A simple model of the tangent/string interaction is proposed. This model reproduces well the behaviour of experimental data, and it may explain why sound quality of the clavichord depends much on the player's ability.

\section{2:00}

3pMUa2. Couchet Harpsichord soundboard vibroacoustics behaviour: An application of the Impact Nearfield Acoustical Holography (IPNAH). Sylvie Le Moyne (UPMC Univ Paris 06, CNRS UMR 7190 Institut Jean Le Rond D'Alembert, 2 Place de la Gare de Ceinture, 78210 Saint Cyr l'Ecole, France, slemoyne@ccr.jussieu.fr), Sandie Le Conte (Musée de la Musique, 
221 avenue Jean Jaures, 75019 Paris, France, sleconte@ cite -musique.fr), François Ollivier (UPMC Univ Paris 06, CNRS UMR 7190 Institut Jean Le Rond D’Alembert, 2 Place de la Gare de Ceinture, 78210 Saint Cyr l'Ecole, France, frol@ccr.jussieu.fr)

The Music Museum in Paris recently acquired a harpsichord made by Ioannes Couchet in 1652 in Anvers. As a masterpiece this instrument is considered as a "National Treasure" and therefore protected. A challenging problematic has risen when its restoration was decided since the aim was to play this instrument again in concert. In the objectives of increasing our understanding of the harpsichord ageing, improve a numerical model currently in process and develop a diagnostic method for conservation, an experimental modal analysis of the soundboard was performed by processing its sound field. A non intrusive method, the Impact Planar Nearfield Acoustic Holography, was used. This technique, developed by the authors, implements the well known inverse method NAH on the basis of the acoustic impulse response field and is well adapted to modal analysis. NAH is performed here in unusual conditions compared to literature, as they are far from the ideal: unbaffled source, low sound pressure level, unusually large measurement distance, preponderance of evanescent waves. An additional challenge was to muffle strings, as they should not be removed nor slackened. However, a very satisfying modal decomposition for $[30-1500 \mathrm{~Hz}]$ bandwidth is obtained. Results are confronted with literature and an energetic analysis is proposed.

3pMUa3. What have they done to the Strads? John McLennan (University of New South Wales, Music Acoustics, School of Physics, NSW 2052 Sydney, Australia, jmc11597@bigpond.net.au), Joe Wolfe (University of New South Wales, Music Acoustics, School of Physics, NSW 2052 Sydney, Australia, J.Wolfe@unsw.edu.au)

Almost every baroque violin, including those of Stradivarius etc, was extensively modified during the conversion to the romantic or modern configuration. What were the acoustic and playing changes? To answer this question, independently of the confounding factors of wood and manufacture, a baroque violin was made and subjected to acoustic and playing tests, before and after (i) replacement of the neck and fingerboard with longer, heavier more inclined parts, (ii) replacing gut with modern nylon-cored strings. Other changes were also made, including bridge style and position, bass bar and soundpost sizes and bow used. Loudness (Saunders plot) was not greatly changed, except for the E string with a long string length. Some acoustic features survived the changes, and professional baroque and modern style players reported that the instrument preserved some of its "personality". Comparisons were made with modern gut strings used by professional baroque players. As well as the acoustic differences, player assessments of bright/dull, full/thin, open/closed, ease of response, evenness, dynamic range are reported. Averaged over all ratings, the players ranked the romantic set up slightly better than the baroque ( $78 \%$ vs $71 \%$ ).

\section{2:40}

3pMUa4. Perceptual studies of violin vibrato. Claudia Fritz (University of Cambridge, Music Faculty, West Rd, CB3 9DP Cambridge, UK, cf291@cam.ac.uk), Jim Woodhouse (University of Cambridge, Dept of Engineering, Trumpington St, CB2 1PZ Cambridge, UK, jw12@cam.ac.uk), Brian Moore (University of Cambridge, Department of Experimental Psychology, Downing Street, CB2 3EB Cambridge, UK, bcjm@cam.ac.uk), Ian Cross (University of Cambridge, Music Faculty, West Rd, CB3 9DP Cambridge, UK, ic108@cam.ac.uk)

Although it seems obvious to violinists that vibrato has a large influence on the perception of violin tone quality, published studies of violin vibrato have mainly concerned its influence on the sound from a scientific point of view, i.e. the characterisation of its time-frequency properties. Work will be described to explore the link between the perception of vibrato notes, the extent of frequency modulation and the level of damping of the resonance modes of the violin. Damping influences the "peakiness" of the frequency response function. The test methodology is based on liveliness ratings and triadic comparisons, where subjects have to select the most similar and the most different pair in each triad (presentation of three sounds). The sounds of the corpus are synthesised using sawtooth waves with frequency modulation, whose amplitude is varied, filtered through a set of admittances corresponding to a reference violin and two modified violins, one with the damping of all modes increased, the other one with the damping decreased.

\section{3:00}

3pMUa5. Performance comparison of violins through experimental force analyses. Enrico Ravina (University of Genoa - Centre of Research on Choral and Instrumental Music (MUSICOS), Via Opera Pia 15 A, 16145 Genova, Italy, enrico.ravina@unige.it), Paolo Silvestri (Univ. of Genoa DIMEC, Via Opera Pia 15 A, 16145 Genova, Italy, p.silvestri@unige.it), Federico Lowenberger (Master Violinmaker, Via Marussig 13, 16100 Genova, Italy, info@ baroqueviolins.com)

The paper describes the most recent experiments developed on violins with different mounting (baroque, classical, modern) and specifically oriented to the dynamic forces analysis in significant points of the instruments. The vibrational behaviour of the soundboards are strongly influenced by the dynamic forces transmitted from the bridge: they are related also to the tension of the strings. An original testbench conceived to forces measurement has been set up: tensions on strings, forces detected under the bridge and deformations on the fingerboard and on the tailpiece are, in particular, monitored by means not intrusive thin-film tactile pressure measurement devices, micro load cells and micro strain gauges. Mapping of forces and strains are acquired in real time playing the instrument; the paper reports comparisons between differently mounted violins, playing notes under different techniques: continuous ("tenuta"), ghost ("strappata"), "detachet". Experiments are developed using different kind of strings (bowels, metallic,...). The results are used as input for experimental dynamic analyses and tests of the soundboard: in addition, they can be correlated to the acoustic response of the instrument.

\section{$3: 20$}

3pMUa6. Shaping and understanding sound: Violin makers, musicians and scientists from Renaissance to Romantism. Anne Houssay (Cité de la musique, 221, avenue Jean Jaurès, 75019 Paris, France, ahoussay@ citemusique.fr)

The training of instrument-makers in Renaissance Italy is linked to a rediscovery of theoretical works on physics and sound as well as to some new tools and skills. Then, the development of physics in the seventeenth and eighteenth century lead the establishment of acoustic as a modern science, with the distinction of partials from harmonics. The "western world" has given explanations on vibrations, modes and then elasticity theories, some of which have been included into general knowledge. By times, links between scientists and makers have developed and have participated to the development of musical instruments. Traditional violin makers are craftspeople, and they construct instruments by choosing step by step between many possibilities from the drawing of the model and the choice of materials, to the cutting out of the shapes to create air volumes and vibrating facings. Their parameters are the dimensions, shapes, weight, elasticity, quality and duration of sounds and notes. The test for judging the results is the playing of the instrument by a very competent musician. We will explore, with a point of view of the historian of techniques how the meeting of different cultures and knowledge have transformed the instruments from the Renaissance to the begining of the 19th century. 


\title{
Session 3pMUb
}

\section{Musical Acoustics: Wind Instruments I}

\author{
Seiji Adachi, Cochair \\ Fraunhofer Institute for Building Physics, Nobelstrasse 12, Stuttgart, 70569, Germany \\ Simon Félix, Cochair \\ Laboratoire d'Acoustique de l'Université du Maine, Avenue Olivier Messiaen, Le Mans, 72085, France
}

\section{Contributed Papers}

$1: 40$

3pMUb1. Individual reed characteristics due to changed damping using coupled flow-structure and time-dependent geometry changing Finite-Element calculation. Rolf Bader (University of Hamburg, Institute of Musicology, Neue Rabenstr. 13, 20354 Hamburg, Germany, R_Bader@tonline.de)

The vibration of a reed of a saxophone is calculated using a 2D model of the mouth cavity, the mouth piece and the reed. The time-dependent FiniteElement calculation takes the flow-structure interaction into account and changes the geometry of the flow according to the reed vibration in time. The model is used to study the flow behaviour, pressure distribution, reed vibration and reaction to disturbances of the system. A constant pressure is assumed at the mouth cavity to simulate the pressure of the player's lungs. During the time-dependent process, an impulse is modeled at the end of the mouth piece travelling back from the end of the tube. The damping of the reed was changed to study the reed behaviour. Hard damped reeds show a simple and stable impulse behaviour causing a clear pressure impulse. When the damping is decreased, the impulse coming back from the end of the tube causes the reed not only to open and close but also to show additional vibrations caused by the interplay of the reed's eigenfrequencies and the pressure acting on the reed. These are more or less independent of the travelling impulse and so are a sound characteristic of the reed itself independent of the played note and used articulation.

\section{2:00}

3pMUb2. Aspects of vibrato and micromodulation in double reed instrument sounds. Michael Oehler (Institute for applied Musicology and Psychology, Saarstrasse 1A, 50677 Koeln, Germany, michael.oehler @ iamp.info), Christoph Reuter (University of Cologne - Musicological Institute, Beethovenstrasse 4, 50674 Koeln, Germany, info@chr-reuter.de)

The perceived naturalness of real and synthesized oboe and bassoon vibrato sounds has been investigated in several perception experiments. The stimuli were generated by means of a currently developed synthesis and analysis framework for wind instrument sounds (first presented at 152nd ASA meeting), based on the pulse forming theory. The framework allows the control of amplitude and frequency parameters at different sound production levels. The stimuli were rated by 60 subjects from "natural" to "unnatural". A conducted ANOVA showed $(\mathrm{p}<.01)$ that the different types of modulation significantly affect the perceived naturalness of vibrato sounds: The synthesized stimuli with combined pulse width and cycle duration modulation (source modulation) are perceived as natural as the real sounds. The subsequently modulated synthesized stimuli (AM and/or FM near the end of the signal path) are perceived significantly less natural $(\mathrm{p}<.01)$. The results support the hypothesis, that source-affected timbre modulation is an important factor for the perceived naturalness of oboe and bassoon vibrato sounds. The use of the developed framework for wind instrument sounds is an alternative method to analyze (micro-)modulation effects. Further investigations may be useful for exploring new sound synthesis algorithms as well as for other experiments in the field of timbre research.
2:20

3pMUb3. The influence of the mean flow on the transmission properties of wind instruments. Andrey R. Da Silva (Schulich School of Music, McGill University, 555 Sherbrooke Street West, Montreal, QC H3A 1E3, Canada, andrey.dasilva@mail.mcgill.ca), Gary Scavone (Schulich School of Music, McGill University, 555 Sherbrooke Street West, Montreal, QC H3A 1E3, Canada, gary@music.mcgill.ca)

The influence of mean flow on the transmission properties of wind instruments has traditionally been neglected due to the range of Strouhal numbers in which this family of instruments normally operate. However, this topic has gained considerable interest among the musical acoustic community during the last decade due to the appearance of new outcomes in research on duct acoustics. Nevertheless, very few contributions have investigated the effect of the mean flow on the transmission properties by taking into account the physical characteristics and dynamic peculiarities of wind instruments. The goal of this work is to present a numerical investigation of the influence of the mean flow on the end correction and on the magnitude of the reflection coefficient for different geometries and Strouhal numbers normally found in wind instruments. The results suggest that, excepting for a few cases, the mean flow can indeed be neglected and the aforementioned transmission properties can be described by the quiescent flow theory.

\section{2:40}

3pMUb4. Effect of bending portions of the air column on the acoustical properties of a wind instrument. Simon Félix (Laboratoire d'Acoustique de l'Université du Maine, Avenue Olivier Messiaen, 72085 Le Mans, France, simon.felix@univ-lemans.fr), Cornelis J. Nederveen (Acacialaan 20, 2641 AC Pijnacker, Netherlands, cjnederv@xs4all.nl), Jean-Pierre Dalmont (Laboratoire d'Acoustique de l'Université du Maine, Avenue Olivier Messiaen, 72085 Le Mans, France, Jean-Pierre.Dalmont @univ-lemans.fr), Joël Gilbert (Laboratoire d'Acoustique de l'Université du Maine, Avenue Olivier Messiaen, 72085 Le Mans, France, joel.gilbert @univ-lemans.fr)

The need to keep long wind musical instruments compact imposes the bending of portions of the air column. Although manufacturers and players mention its effects as being significant, the curvature is generally not included in physical models and only a few studies, in only simplified cases, attempted to evaluate its influence. The aim of our study is to quantify the influence of the curvature by modelling the wave propagation in an air column with a multimodal formalism. In a duct with a circular cross-section and a finite curvature, two infinite sets of coupled first-order differential equations are constructed for the components of the pressure and axial velocity, projected on the local transverse modes. From these an impedance matrix is defined, which can be easily calculated, particularly when considering a duct with a piecewise constant curvature. Influence of the curvature on the input impedance, effective length, or playing frequencies is then quantified, displaying notably a dependence to frequency such that, compared to an equivalent straight tube, the shift in resonance frequencies in a tube with bent sections is not always positive, as generally stated. Results are independently corroborated by numerical - finite differences computations. 
3pMUb5. Numerical investigations of the bassoons aeroacoustic. Andreas Richter (Technische Universität Dresden, Institute for Aerospace Engineering, 01062 Dresden, Germany, andreas.richter4@tu-dresden.de), Roger Grundmann (Technische Universität Dresden, Institute for Aerospace Engineering, 01062 Dresden, Germany, roger.grundmann@tu-dresden.de)

Solving the compressible, unsteady Navier-Stokes equations allows us to track single traveling waves in conjunction with a nonuniform superposed mean flow. Significant physical phenomena like wave steepening, viscothermal losses and acoustic streaming can be investigated. This may be helpful to understand the acoustical characteristics of different geometries, especially in musical woodwind instruments. Since acoustic perturbations are usually small compared to the driving pressure, the solution demands highorder accuracy and appropriate boundary conditions. We present a collection of our numerical results based on two- and threedimensional simulations of the aeroacoustic behavior inside the bassoon. This includes both simulations of the whole instrument under real playing conditions and also numerical studies of subsections of the instrument like tone holes. Here especially viscothermal effects are analyzed. Nonlinear effects like acoustic streaming which is primarily present in the crook of the instrument are also investigated and discussed. Flow field measurements based on Particle Image Velocimetry and pressure measurements inside and outside the instrument are performed to validate the earned results and show a good agreement between measurements and numerical results.
3pMUb6. Analysis of the timbre of Slovak folk reed aerophones using source-filter model. Milan Rusko (Institute of Informatics of the Slovak Academy of Sciences, Dubravska cesta 9, 84507 Bratislava, Slovakia, milan.rusko@savba.sk)

Bagpipes are the only group of traditional folk reed instruments that have survived in Slovak folk culture until now. Unlike Scottish highland pipe, gaita, cornemuse etc., all the types of Slovak bagpipes use single reeds in both chanters and drones. Moreover the horns of the drones are partly closed with a plate having only a small opening for air output and sound radiation. This forms a Helmholz-like resonator - acoustic filter having a strong influence on the acoustic and aesthetic properties of the sound. The ideal of the sound timbre of these pipes is "less open" (compare to open and closed vowels in speech) and, in spite of its relatively high loudness, "less agressive" in comparison to pipes with open horns. This paper presents our approach to study the ideal of sound timbre of the Slovak bagpipe using a source-filter all-pole model. To identify resonance frequencies/formants, we applied inverse filtering technique using discrete linear prediction. Some properties of the source (reed) were studied using parameters like amplitude qutient and open quotient. The possible relations between timbre of speech sounds (vowels) and the timbre of the sound of musical instruments are discussed.

\title{
Session 3pNSa
}

\section{Noise, ASA Committee on Standards, and EURONOISE: Sleep Disturbances and Other Health Effects}

\author{
Lily M. Wang, Cochair \\ University of Nebraska - Lincoln, 1110 S. 67th St., Omaha, NE 68182-0681, USA \\ Barbara Griefahn, Cochair \\ Institute for Occupational Physiology, Ardeystr. 67, Dortmund, 44139, Germany \\ Jacques Lambert, Cochair \\ Laboratoire Transport et Environnement, INRETS, 25, Avenue François Mitterrand, F-69675 BRON cedex, France
}

Invited Paper

$1: 40$

3pNSa1. Associations between road traffic noise level, road traffic noise annoyance and high blood pressure in the HYENA study. Wolfgang Babisch (Federal Environmental Agency, Corrensplatz 1, 14195 Berlin, Germany, wolfgang.babisch @uba.de), Danny Houthuijs (The National Institute For Public Health and Environmental Protection, PO Box 1, 3720 B A Bilthoven, Netherlands, danny.houthuijs@ @rivm.nl), Goran Pershagen (Institute of Environmental Medicine, Karolinska Institutet, Box 210, 17177 Stockholm, Sweden, goran.pershagen@ki.se), Klea Katsouyanni (Department of Hygiene and Epidemiology, Medical School, University of Athens, 75 Mikras Asias Str, 11527 Athens, Greece, kkatsouy@med.uoa.gr), Manolis Velonakis (Laboratory of Prevention, Nurses School, University of Athens, 123 Papadiamadopoulou St, 11527 Athens, Greece, evelonak@nurs.uoa.gr), Ennio Cadum (Piedmont Regional Environmental Protection Agency, Via Sabaudia 164, 10095 Grugliasco (TO), Italy, e.cadum @arpa.piemonte.it), Lars Jarup (Imperial College London, Norfolk Place, W2 1PG London, UK, 1.jarup@imperial.ac.uk)

The HYENA study is a multi-centred study regarding the effects of aircraft noise and road traffic noise on blood pressure (BP) which was funded by the European Community. Study subjects were 4,861 males and females aged between 45 and 70 years, who had lived for at least 5 years in the vicinity of any of six major European airports. Aircraft noise contours and road traffic noise levels were modelled using the Integrated Noise Model (INM) and national calculation methods. The noise levels were linked to each participant's home address (most exposed façade) using graphical information systems (GIS). Noise annoyance was assessed using the 11-point ICBEN scale. High blood pressure was determined by measurements of systolic and diastolic blood pressure, anti-hypertensive medication and self-reported doctor diagnosed hypertension. The focus here is on the effects of road traffic noise. The road traffic noise level 
(LAFm, 24 hours) and noise annoyance due to road traffic noise were both significantly associated with high blood pressure. The association between road traffic noise and high blood pressure was stronger for the road noise level than for the road noise annoyance (90th percentile vs. 10th percentile of the exposure distributions).

\section{Contributed Paper}

\section{2:00}

3pNSa2. Computing Number of People Awakened by Aircraft Operations Noise. Nicholas P. Miller (Harris Miller Miller \& Hanson Inc., 77 South Bedford Street, Burlington, MA 01803, USA, nmiller@hmmh .com)

The acoustics literature documents a number of field studies in which aircraft noise was measured in people's bedrooms while their awakening was simultaneously monitored. Nearly all the field studies produced a doseresponse relationship between noise and an awakening response. These dose-response relationships generally show good agreement with each other. Virtually without exception, however, these dose-response relationships per- tain to (1) the indoor noise dose produced by a single aircraft flyover and (2) the chances that the noise dose will awaken an average person. These doseresponse relationships are too limited for application to a full night of operations and to a realistic population of varying individual sensitivities to noise-induced awakenings. The dose-response relationships do not account for multiple aircraft exposures during the night or for person-to-person variation in how deeply different people sleep. This presentation first briefly reviews a method previously reported for applying the study data to a full night of operations, accounting for time of night and for individual sensitivity to awakening. It then compares percent of population awakened for realistic situations, and shows the effects of including or excluding time of night or individual sensitivities to awakening.

\section{Invited Paper}

3pNSa3. Determination of noise sensitivity within an internet survey using a reduced version of the Noise Sensitivity Questionnaire. Barbara Griefahn (Institute for Occupational Physiology, Ardeystr. 67, 44139 Dortmund, Germany, griefahn@ifado .de)

The Noise Sensitivity Questionnaire (NoiSeQ), that determines noise sensitivity as a moderator of annoyance globally and separately for different everyday activities is with 35 items too long for extended social surveys. This study aimed at the development of a shorter version. Using 429 questionnaires (266 women, $163 \mathrm{men}, 16-74 \mathrm{yrs}$ ) three factor analyses were performed leading to the NoiSeQ-R that consists of the three subscales 'Sleep', 'Habitation', and 'Work' with 4 items each. The scores are normally distributed, internal consistency is $\alpha=0.87$ and test-retest reliability varies between $\mathrm{rtt}=0.66$ and 0.74 depending on the time gaps that varied between 1 to 37 months. To test the validity the NoiSeQ-R was applied to a field study where 190 residents (102 males, 88 females, 17-80 years, median: 51 years) in the vicinity of a large airport rated their chronic annoyance (for the previous 12 months) and their actual annoyance hourly during four consecutive days. As expected, noise sensitivity did not correlate with the individual noise load but significantly with annoyance and with age. Thus the NoiSeQ-R, which is available in ten languages, is regarded as a reliable and valid instrument which can be easily applied in even extended surveys.

\section{Contributed Paper}

\section{2:40}

3pNSa4. A case study on the validation of models that predict the impact of aircraft noise on sleep. Sarah McGuire (Ray W. Herrick Lab., School of Mechanical Engineering, Purdue Univ., 140 S. Martin Jischke Drive, West Lafayette, IN 47907-2031, USA, mcguires@purdue .edu), Patricia Davies (Ray W. Herrick Lab., School of Mechanical Engineering, Purdue Univ., 140 S. Martin Jischke Drive, West Lafayette, IN 47907-2031, USA, daviesp@ecn.purdue.edu)

The impact of aircraft noise on sleep disturbance is not accurately assessed by cumulative noise metrics that are often used to predict community impact, such as $L_{\mathrm{dn}}$, because sleep disturbance is highly dependent on the noise level of individual events. Most existing sleep disturbance models are dose-response relationships that relate the noise level of individual events, as measured by $\operatorname{SEL}(A)$ or $L_{A_{\max }}$ to the percent awakened, and independence of responses to individual events is assumed. There are a few models that also incorporate additional parameters such as time of night and noise sensitivity, and some that predict the impact of noise on sleep structure. In order to determine whether a model should be used as a general aircraft noise sleep disturbance prediction tool, its performance in a variety of situations must be evaluated. Data was collected from a number of sleep disturbance studies and comparisons were made between responses and model predictions of awakenings and sleep stages. The sufficiency of the collected data for estimating the parameters of more complex models of sleep disturbance is also discussed.

\section{Invited Paper}

3pNSa5. Effects of a hearing aid noise management feature on user perception and performance under noise. Lauren M. Ronsse (University of Nebraska - Lincoln, 1110 S. 67th St., Omaha, NE 68182-0681, USA, lronsse@ mail.unomaha.edu), Lily M. Wang (University of Nebraska - Lincoln, 1110 S. 67th St., Omaha, NE 68182-0681, USA, LWang4@UNL.edu)

This paper presents the effects of a hearing aid noise management feature on user perception and performance under noise. Hearingimpaired subjects were exposed twice to seven different noise signals which simulate common mechanical system noise, once with the noise management feature enabled and once disabled. The seven noise signals vary in terms of level and spectral quality, but are all within the range of background noise conditions found in commercial offices. Performance is gauged on three types of tests (math, verbal and typing), while subjective perception is measured via a subjective questionnaire. The results of this investigation will show if the hearing aid noise management feature reduces detrimental effects caused by background noise. Additionally, the data from hearing- 
impaired subjects are compared to those from normal-hearing persons to assess if significant differences are present between the two groups. If so, they may lead to the development of different standards for noise criteria levels in spaces designed for the hearingimpaired. [Work supported by a Univ. of Nebraska Layman Award and an ASHRAE Graduate Student Grant-in-Aid]

\title{
Contributed Paper
}

3:20

3pNSa6. Noise and health in the Greater Rotterdam Area. Henk Wolfert (DCMR EPA, P.O. Box 843, sGravelandseweg 565, 3100AV Schiedam, Netherlands, henk.wolfert@dcmr.nl)

In the Greater Rotterdam Area a second study to Noise and Health has been carried out in 2007 and 2008. In 2003 a first study was carried out and that study reported that around 12 percent of the people living in the Greater Rotterdam Area were highly annoyed by noise mainly caused by traffic. The number of people sleep was disturbed and suffered high blood pressure were reported and amounted to 6 percent for sleep disturbance and 3 percent were suffering hypertension. The 2003 study took place within the regularly framework of the Rotterdam Regional Council of Governments Environmental Monitoring program, a program that reports yearly the environmental performance indicators in the Greater Rotterdam Area. Beside this yearly report a theme report is published. Last year's theme reports were published about Air Quality and Energy. In 2007 the board of this program decided that in 2008 the theme should be Noise and Health. The 2008 study is not only an update of the 2003 study but is more detailed and more comprehensive as well, and based on recent insights in health effects, caused by long lasting noise too.

WEDNESDAY AFTERNOON, 2 JULY 2008

ROOM 251, 2:00 TO 3:40 P.M.

\section{Session 3pNSb}

\section{Noise, ASA Committee on Standards, and EURONOISE: Prominent Discrete Tones I}

\author{
Robert Hellweg, Cochair \\ 13 Pine Tree Road, Wellesley, MA 02482, USA \\ Lothar Schmidt, Cochair \\ Currenta GmbH \& Co. OHG, Building F1, Dormagen, 41538, Germany
}

\section{Invited Papers}

\section{2:00}

3pNSb1. Comparison of various procedures for the assessment of prominent discrete tones using a large number of sound samples. Lothar Schmidt (Currenta GmbH \& Co. OHG, Building F1, 41538 Dormagen, Germany, lothar.schmidt@currenta.de), Dirk Sagemuehl (Kattenbachstr. 77a, 51467 Bergisch-Gladbach, Germany, DSagemuehl@aol.com)

The procedures from the standards ISO 1996-2 and DIN 45681 as well as the two procedures (TNR and PR) from ISO 7779 are compared. In the first section, characteristic features of the procedures (e.g. signal analysis parameters, degree of automation and characteristics of the prominent discrete tone analysis) are compared. In the second section, the procedures are applied to approximately 70 sound samples. Besides natural sounds (e.g. from machines), synthetic sounds (e.g. peak in a trough) are also used. The results are compared against assessments of the sounds by human subjects. The results of the comparisons are discussed, with special attention paid to cases where significant deviations occur. The third section looks at the results of the comparisons under the aspect of requirements for a standard (e.g. robustness, precision). Overall it was determined that none of the procedures investigated provides optimal results for all sound samples.

3pNSb2. Prominent tones in noise - Proficiency testing among 30 laboratories of the ISO 1996-2 Annex C method and its predecessors. Torben Holm Pedersen (Delta Acoustics \& SenseLab, Venlighedsvej 4, 2970 Hørsholm, Denmark, thp@delta.dk)

Since 1979 the Danish Environmental Protection Agency's Reference laboratory for noise measurements have arranged proficiency testing for the around 30 approved Danish laboratories that are measuring environmental noise. Many of these "comparison measurements" have included objective analysis of the prominence of audible tones according to the Joint Nordic Methods which are the predecessors and very similar to the method described in ISO 1996 part 2 Annex C. This paper gives examples on (computerized) analyses of the samples from these proficiency tests and states the uncertainties that can be expected when a number of laboratories with different experience and different types of equipment analyze the same samples. The samples will be available for future reference and can be downloaded from DELTA's homepage. 
3pNSb3. Updates on Prominent Discrete Tone Procedures in ISO 7779, ECMA 74, and ANSI S1.13. Robert Hellweg (13 Pine Tree Road, Wellesley, MA 02482, USA, Hellweg@HellwegAcoustics.com)

There are two alternative procedures for determining if a tone is prominent, i.e. a "prominent discrete tone" in ISO 7779, ECMA 74 and ANSI S1.13: the Tone to Noise Ratio (TNR) method and the Prominence Ratio (PR) method. Both of these procedures have been used by the computer industry for more than a decade. Recent revisions to these procedures have refined the calculation of the critical bandwidth and modified the both procedures for low level tones. This paper describes these changes and the rationale behind them. Examples are presented that demonstrate the features of each procedure. The paper presents an overview on the applicability and limitations of each procedure.

\section{3:00}

3pNSb4. Issues in the evaluation of the tonality of nonstationary sounds containing time- varying harmonic complexes. Patricia Davies (Ray W. Herrick Lab., School of Mechanical Engineering, Purdue Univ., 140 S. Martin Jischke Drive, West Lafayette, IN 47907-2031, USA, daviesp@ecn.purdue.edu), Shashikant R. More (Ray W. Herrick Lab., School of Mechanical Engineering, Purdue Univ., 140 S. Martin Jischke Drive, West Lafayette, IN 47907-2031, USA, shashi@purdue.edu)

Nonstationary sounds that contain broadband noise and tone complexes whose fundamental frequency varies with time are encountered frequently. Aircraft flyovers and machines shifting operating speeds are examples. The tonalness of such sounds is a factor in how annoying they are. Most metrics used to quantify the tonalness of sounds have been developed for stationary sounds and are based on an analysis of spectra. Contributions from individual sinusoids are estimated and either summed or the maximum is taken. When sounds vary, spectral estimation can be problematic due to the conflicting needs to reduce the variance of the estimate, maintain high frequency resolution, and generate spectra at a rate where the varying tonal behavior can be tracked sufficiently. Harmonic complexes are often perceived holistically, having one dominant pitch, thus treating sinusoidal components independently and summing may not be appropriate. Tests conducted to analyze the tonalness of harmonic complexes in noise are described, and an analysis of aircraft flyovers is used to illustrate the issues that need to be addressed when estimating the tonalness of complex time-varying sounds.

\section{3:20}

3pNSb5. Implementation of ISO 1996-2 (2007) pure tone assessment in a sound level meter. Charles Greene (Brüel \& Kjær, Skodsborgvej 307, 2850 Nærum, Denmark, cgreene@bksv.com), Douglas Manvell (Brüel \& Kjær, Skodsborgvej 307, 2850 Nærum, Denmark, dmanvell@bksv.com), Matthias Scholz (Brüel \& Kjær, Skodsborgvej 307, 2850 Nærum, Denmark, mscholz@bksv.com) , Anne Lin Enggaard (Nordsjællands Akustik, Skræntevej 13, Holløselund, 3210 Vejby, Denmark, ale@nordsjaellandsakustik.dk)

Noise can be said to be tonal if it contains a distinguishable, discrete, continuous note. This may include a whine, hiss, screech, hum, etc., and any such subjective finding is open to discussion when reported. This is important when it is considered that the likelihood of a noise-provoking complaint depends on its relative level to background, and whether or not it has certain audible characteristics. Fortunately, ISO 1996-2 (2007) provides objective FFT (engineering) and 1/3-octave band (survey) assessment procedures to be used to verify the presence of audible tones if their presence is in dispute. Brüel \& Kjær has implemented the methodology of ISO 1996-2 in relation to assessing the audibility of prominent discrete tones in environmental noise into its innovative 2250 and 2270 hand-held analysers. This paper describes the implementation in hand-held instrumentation. 


\title{
Session 3pNSc
}

\section{Noise and EURONOISE: Car Acoustics I}

\author{
Luc Mongeau, Cochair \\ McGill University, 817 Sherbrooke St. West, Montreal, QC H3A 2K6, Canada \\ Virginie Maillard, Cochair \\ RENAULT, Groupe Acoustique, Technocentre, 1 avenue du Golf, 78288 Guyancourt Cedex, France
}

\section{Contributed Paper}

\begin{abstract}
2:20
3pNSc1. Effects of notches on span wise correlation of surface pressure fluctuations downstream of a wall-mounted spoiler. Paloma Mejia (Purdue University, 140 S. Intramural Dr., West Lafayette, 47906, USA, umaphuyu@gmail.com), Jong Beom Park (McGill University, 817 Sherbrooke St. West, Montreal, QC H3A 2K6, Canada, jong.b.park @ mail.mcgill.ca), Luc Mongeau (McGill University, 817 Sherbrooke St. West, Montreal, QC H3A 2K6, Canada, luc.mongeau@mcgill.ca)

Leading edge spoilers are widely-used for suppressing flow-induced cavity resonance such as buffeting due to open sunroofs in moving cars. Spoilers deflect the grazing flow over the opening into a region of greater flow velocity, thereby increasing the critical velocity. Notched spoilers have

been observed to enhance resonance suppression while moving the flow reattachment region upstream, resulting in a decreased drag. The mechanisms involved in the effectiveness of the notched spoiler were investigated experimentally and numerically. Static and dynamic pressures on the surface behind a wall mounted notched spoiler were measured, and the spatial correlations of the measured pressures were compared to those for a spoiler without notches. The span wise pressure correlation was decreased by the presence of the notches, suggesting a breakdown of the span wise leading vortices predominantly responsible for the cavity excitation. Numerical flow simulations were performed using the lattice Boltzmann method (LBM) with turbulence modeling. Surface pressure results were compared with experimental data to better highlight surface pressure trends.
\end{abstract}

\section{Invited Papers}

3pNSc2. Simulation of vehicle interior wind noise at low frequencies: a case study. Robert Powell (Ford Motor Company, EVB, MD X-19, Rm 1EB08, 20800 Oakwood Blvd, Dearborn, MI 48121, USA, rpowell7@ford.com), Bijan Khatib-Shahidi (Ford Motor Company, EVB, MD X-19, Rm 1EB08, 20800 Oakwood Blvd, Dearborn, MI 48121, USA, bshahidi@ford.com)

Quietness of passenger vehicle interiors has become a critical-to-quality metric in designing modern passenger vehicles. At the same time, the necessity of bringing fresh designs to market quickly has greatly compressed the development time available to achieve the desired refinement of interior noise and vibration. This has forced manufacturers to place increasing responsibility on analytical simulations in developing countermeasures for noise problems. One aspect of car acoustics that has not received very much attention from the simulation community is the wind noise created by external air flow at speed. Significant contributions to interior loudness can come from underbody air flow, while improvements in simulation efficiency of Computational Fluid Dynamics (CFD) flow models and Finite Element Analysis (FEA) vehicle vibration and acoustic models now make it feasible to analytically simulate interior noise caused by wind excitation. This lecture describes a case study where existing vehicle models were adapted to first build a wind load case from $\mathrm{CFD}$, and then to apply it in estimating and reducing interior noise in FEA. Topics to be covered include: spatial discretization of continuous panel pressures, application of random loads to deterministic vehicle FEA models and diagnostic imagery for visualizing noise and vibration responses.

\section{3:00}

3pNSc3. On the use of linear aero-acoustic models. Mats Abom (KTH-The Marcus Wallenberg Laboratory, Teknikringen 8, 100 44 Stockholm, Sweden, matsabom@kth.se)

The classical theory of aero-acoustics is based on Lighthills acoustic analogy which essentially leads to a wave equation with a source term defined by the flow field. This source term is assumed unaffected by the acoustic field and the resulting model can be seen as a linear model which, e.g., for duct acoustic problems can be formulated as an acoustic multi-port. Such linear aero-acoustic models can be applied to most aero-acoustic problems occurring in engineering practice with the exception of whistling, i.e., situations where the source term is affected by the acoustic field. At KTH this methodology has been applied to a number of applications over the years ranging from cooling and ventilation fans to flow generated noise from ducted orifices (valves) and air terminal devices. In this paper the experience from these works is summarized and the experimental techniques developed at KTH to characterize linear aero-acoustic sources are described. Recent efforts to extract linear aero-acoustic models from numerical calculations are also addressed. 


\title{
Contributed Paper
}

$3: 20$

3pNSc4. Modeling and characterization of rattle noise encountered in an automotive environment. Ludovic Desvard (Renault, Technocentre, 1 avenue du Golf, 78288 Guyancourt, France, ludovic.desvard@renault .com), Nacer Hamzaoui (LVA, INSA de Lyon, Bat. Saint-Exupéry, 25 bis avenue Jean Capelle, 69621 Villeurbanne, France, nacer.hamzaoui@insa -lyon.fr), Jean-Marc Duffal (Renault, Technocentre, 1 avenue du Golf, 78288 Guyancourt, France, jean-marc.duffal@ renault.com)

In an automotive cockpit, rattle noises deal with all noise due to normal impacts which radiate as annoying noises for customers. The state of the art shows studies focused on one automotive subsystem, describing the specific associated physic. The method proposed, in this study, is to generate auto- motive rattle noises using simple geometries. The interest of this approach is to know all the parameters of the system (impact location, material, geometries, excitation...) and to precisely describe the physical phenomena related to the apparition of rattle noises. Firstly an experimental bench was proposed to generate rattle noises using simple geometries. These rattle noises are similar to those encountered in an automotive environment. This experimental approach allows to measure the vibrational behaviour and the radiated sound of the system under a random impact excitation. Then an analytical model, which describes the experimental approach, is developed to predict the radiated sound, knowing the displacement of the impactor. Numerical and experimental results are compared. Finally, thanks to the experimental approach, a database of rattle noises is created and used to find metrics to quantify the annoyance.

WEDNESDAY AFTERNOON, 2 JULY 2008

ROOM 351, 1:40 TO 3:40 P.M.

Session 3pPAa

Physical Acoustics: Quantum Acoustics I

\author{
Michel De Billy, Cochair \\ Institut Jean le Rond d'Alembert / UMR 7190, 2, place de la Gare de Ceinture, Saint Cyr l'Ecole, 78210, France \\ Walter Lauriks, Cochair \\ Lab ATF, Katholicke Universiteit Leuven, Celestijnenlaan 2000, Leuven, B-3001, Belgium
}

\section{Contributed Papers}

\begin{abstract}
1:40
3pPAa1. Phonon dispersion in graphene. Leonid A. Falkovsky (Landau Institute for Theoretical Physics, Kosygina 2, 119334 Moscow, Russian Federation, falk@itp.ac.ru)

Taking into account constraints imposed by the lattice symmetry, we calculate analytically [1] the phonon dispersion for graphene with interactions between first and second neighbors. We find that the out-of-plane (bending) modes are not coupled with in-plane modes and described only with two force constants, one of which is determined by the corresponding Raman frequency and another by the smallest elastic constant C44. In contrast to calculations by Saito et al, we find the linear dispersion of the bending (outof-plane) mode around the $\Gamma$ point with a small but finite sound velocity $=1.57 \mathrm{~km} / \mathrm{s}$. The sound velocity of this mode is very sensitive to small variations of the force constants. The sound velocities of in-plane modes are $=20.3 \mathrm{~km} / \mathrm{s}$ and $=13.1 \mathrm{~km} / \mathrm{s}$. Because of the lack of information for graphene, we compare the present theory with experiments on graphite. The low phonon frequencies in the critical points turn out less than their values in graphite, since the atoms in graphene are more free to move in the outof-plane direction in comparison with graphite. Accuracy of the comparison can be estimated using the value of the observed splitting of the ZA and ZO' modes in graphite which is around $1301 / \mathrm{cm}$. [1] L.A. Falkovsky, condmat/0702409.
\end{abstract}

\section{2:00}

3pPAa2. Mechanical (acoustic-like) wave propagation along a vortex array in the superconducting heterostructure. Bogdan $\mathrm{T}$. Maruszewski (Poznan University of Technology, Institute of Applied Mechanics, ul. Piotrowo 3, 60-965 Poznan, Poland, bogdan.maruszewski @ put.poznan.pl), Andrzej Drzewiecki (Poznan University of Technology, Institute of Applied Mechanics, ul. Piotrowo 3, 60-965 Poznan, Poland,
andrzej.drzewiecki@put.poznan.pl),Roman Starosta(Poznan University of Technology, Institute of Applied Mechanics, ul. Piotrowo 3, 60-965 Poznan, Poland, roman.starosta@put.poznan.pl)

Magnetic flux can penetrate the type - II superconductor in the form of Abrikosov vortices (also called flux lines, flux tubes or fluxons) each carrying a quantum of magnetic flux. These tiny vortices of supercurrent tend to arrange themselves in a triangular or quadratic flux-line lattice. Since the vortices are formed by the applied magnetic field, around of each of them the supercurrent flows. Moreover, there also exist some Lorentz force interactions among them. Those interactions form an origin of an additional mechanical (stress) field occurring in the type-II superconductor. The paper deals with an analysis of elastic (acoustic-like) wave propagation solely along vortices in a heterostructure consisted of the superconducting layer put on the superconducting substrate. Dispersion and the amplitude distribution of those waves in the vortex field existing in that structure has been presented.

\section{2:20}

3pPAa3. Amplitude distribution of magnetoelastic waves propagating in a vortex field in a superconducting layer. Bogdan $T$. Maruszewski (Poznan University of Technology, Institute of Applied Mechanics, ul. Piotrowo 3, 60-965 Poznan, Poland, bogdan.maruszewski @ put.poznan.pl), Andrzej Drzewiecki (Poznan University of Technology, Institute of Applied Mechanics, ul. Piotrowo 3, 60-965 Poznan, Poland, andrzej.drzewiecki@put.poznan.pl), Roman Starosta (Poznan University of Technology, Institute of Applied Mechanics, ul. Piotrowo 3, 60-965 Poznan, Poland, roman.starosta@put.poznan.pl)

Magnetic field enters the type - II superconducting body along a discrete arrangement of magnetic vortex lines. In the dynamic case when the magnetic field vary in time, around each such a line a supercurrent flows. So, the vortices interact one to another with the help of the Lorentz force forming this way a new mechanical field of elastic properties. Moreover, those lines arrange themselves in a triangular or quadratic lattice. Such a set is observed 
if the intensity of the applied to the material magnetic field is close to its lower limiting value. The paper aims at investigating amplitude distributions of magnetoelastic waves propagating solely in the vortex field of the superconducting layer. Our attention have been focused on the applied magnetic field intensity influence on those amplitudes for various wave frequencies.

\section{2:40}

3pPAa4. Acoustical dissipation due to phonon-phonon interaction, thermoelastic loss and dislocation damping in $\mathrm{MnO}$ and $\mathrm{CoO}$. Rishi $\mathrm{Pal}$ Singh (Banaras Hindu University, 221005 Varanasi, India, rishisingh80 @ rediffmail.com), Manish Pratap Singh (Banaras Hindu University, 221005 Varanasi, India, mps_bhu@yahoo.co.in), Rajendra Kumar Singh (Department of Physics, Banaras Hindu University, 221005 Varanasi, India, rksingh_17@rediffmail.com)

The temperature dependent acoustical dissipation due to phonon-phonon (p-p) interaction, thermo-elastic mechanism and dislocation damping due to screw and edge dislocations have been evaluated in $\mathrm{MnO}$ and $\mathrm{CoO}$ in $\mathrm{fcc}$ ( $\mathrm{NaCl}-\mathrm{B} 1$ type) phase, in a wide temperature range $50 \leqslant \mathrm{~T} \leqslant 500 \mathrm{~K}$ for longitudinal and shear modes of propagation along three crystallographic directions viz. $<100>,<110>$ and $<111>$. Electrostatic and Born repulsive potentials were used to obtain second order and third order elastic constants (SOEC and TOEC), taking interactions up to next nearest neighbours. The SOEC and TOEC obtained at diffrent temperatures have been used to obtain gruneisen parameters and non-linearity or anisotropic parameters which in turn were used to evaluate $(\alpha / \mathrm{f} 2)$ for longitudinal and shear waves. Specific heat (as function of Debye temperature), hardness parameter, lattice parameter have been used as input data. It has been found that $(\alpha / \mathrm{f} 2)$ increases with temperature and the acoustical dissipation is mainly influenced by $p-p$ interaction. The possible implications of results have been discussed.

\section{3:00}

3pPAa5. Numerical and experimental results on sonic band gaps in 1-D phononic crystals with a symmetric stub. Michel De Billy (Institut Jean le Rond d'Alembert / UMR 7190, 2, place de la Gare de Ceinture, 78210 Saint Cyr l'Ecole, France, mdebilly@univ-paris-diderot.fr), Anne-Christine Hladky-Hennion (IEMN, UMR CNRS 8520, avenue Poincaré, BP 60069, 59652 Villeneuve d'Ascq, France, Anne-Christine.Hladky@isen.fr), Jérôme Vasseur (IEMN, UMR CNRS 8520, avenue Poincaré, BP 60069, 59652 Villeneuve d'Ascq, France, jerome.vasseur@univ-lille1.fr), Bahram Djafari-Rouhani (IEMN, UMR CNRS 8520, avenue Poincaré, BP 60069, 59652 Villeneuve d'Ascq, France, Bahram.Djafari-Rouhani@univ-lille1.fr)

The wave propagation in periodic systems has received a great deal of attention during the last years. By analogy with the studies driven on photonic crystals, many works were conducted on phononic crystals. In this pa- per the propagation of elastic waves through a one dimensional chain of beads with grafted stubs is experimentally as well as numerically investigated. The results obtained by both approaches are well correlated and show that the stub introduces a dip in the spectral response of the chain. The numerical analysis shows that this dip is due to the excitation of a stub mode that cancels the transmission from one extremity of the chain to the other. The position and the shape of the dip in the response are related to the geometry and nature of the stub. The results show that it is possible to adjust the position of the dip and open potential applications of these structures for filtering or demultiplexing. Finally first results on periodically grafted stubs in the chain are presented.

$$
\text { 3:20 }
$$

3pPAa6. Evidence for vortex transport by surface acoustic waves in a high-Tc superconductor. Carsten Hucho (Paul-Drude-Institut, Hausvogteiplatz 5-7, 10117 Berlin, Germany, hucho@pdi-berlin.de), Munise Rakel (Paul-Drude-Institut, Hausvogteiplatz 5-7, 10117 Berlin, Germany, rakel@pdi-berlin.de), Arno Wirsig (Paul-Drude-Institut, Hausvogteiplatz 5-7, 10117 Berlin, Germany, wirsig@pdi-berlin.de), Fabian Jachmann (Paul-Drude-Institut, Hausvogteiplatz 5-7, 10117 Berlin, Germany, jachmann@pdi-berlin.de)

The interaction of surface acoustic waves (SAW) with the magnetic vortex system in $\mathrm{YBa} 2 \mathrm{Cu} 3 \mathrm{O} 7$ is investigated. A 100nm YBCO film is deposited on a piezoelectric substrate and structured for electrical 4-point measurements. Interdigital transducers are fabricated on the same substrate. When applying an external magnetic field perpendicular to the film surface a SAW-induced dc-voltage perpendicular to the acoustic sound-path is observed. This is interpreted as consequence of directed dragging of vortices by the SAW induced dynamic pinning structure [1]. The piezoacoustic wave acts as a conveyor for mobile flux quanta. Additional ac-dc-conversion as result of the nonlinear current-voltage characteristics close to the superconducting transition temperature can be resolved and separated. In order to observe the sound-induced vortex motion directly, the flux distribution is analyzed magnetooptically. Magnetooptic imaging allows for time resolved analysis of flux distribution. Quantitative analysis of changes in the magnetization distribution when acoustic driving fields are applied is carried out. The influence of piezoacoustic waves on the pinning properties and soundinduced depinning is discussed. [1] F. Jachmann and C. Hucho, Sol. State Comm., 142 (4) (2007), 212 


\title{
Session 3pPAb
}

\section{Physical Acoustics: General Topics in Nonlinear Acoustics I}

\author{
Thomas Matula, Cochair \\ Center for Industrial and Medical Ultrasound, Applied Physics Lab., University of Washington, 1013 NE 40th St., \\ Seattle, WA 98105, USA \\ Murray S. Korman, Cochair \\ Physics Dept., United States Naval Academy, Chauvenet Hall Room 295, 572 C Holloway Road, Annapolis, MD 21402, USA
}

\section{Contributed Papers}

\begin{abstract}
2:00
3pPAb1. Atomization and deviation of cylindrical water jets in a transverse acoustic field. Jean-Baptiste Carpentier (Coria UMR 6614, avenue de l'Université, 76800 Saint-Etienne-du-Rouvray, France, jean-baptiste.carpentier@univ-rouen.fr), Françoise Baillot (Coria UMR 6614, avenue de 1'Université, 76800 Saint-Etienne-du-Rouvray, France, baillot@coria.fr), Jean-Bernard Blaisot (Coria UMR 6614, avenue de l’Université, 76800 Saint-Etienne-du-Rouvray, France, blaisot@coria .fr), Christophe Dumouchel (Coria UMR 6614, avenue de l'Université, 76800 Saint-Etienne-du-Rouvray, France, dumouchel@coria.fr)

This work deals with a particular breakup mode experienced by cylindrical liquid jets when submitted to an intense transverse acoustic wave. Experiments on low speed water jets $(<1 \mathrm{~m} / \mathrm{s})$ of diameters $3 \mathrm{~mm}$ and $6 \mathrm{~mm}$ show that sound waves with a frequency ranging from $500 \mathrm{~Hz}$ to $1800 \mathrm{~Hz}$ can produce bulges along the jet. When the sound level is high enough, these bulges can trigger an effective atomization mechanism where the jet flattens as a liquid sheet before disintegrating. Sound field can also induce steady deviation of the jet. Both phenomena are theoretically studied. A first model, which treats bulges as outward marks of one particular mode of vibration of the liquid column, is proposed. This model leads to a criterion for the onset of atomization that satisfactorily agrees with experimental observations of the present work. A second analysis identifies deviations as radiation pressure effects. It predicts the direction of experimental deviations with success.
\end{abstract}

\section{2:20}

3pPAb2. Numerical and experimental observations of azimutal shock waves. Régis Marchiano (Institut Jean Le Rond d'Alembert, Université Pierre et Marie Curie, Boites 161 et 162, 4 place Jussieu, 75252 Paris Cedex 05, France, marchi@1mm.jussieu.fr), Jean-Louis Thomas (Centre National de la Recherche Scientifique, Institut des NanoSciences de Paris, Université Pierre et Marie Curie, 4 place Jussieu, 75252 Paris Cedex 05, France, thomasj1@ccr.jussieu.fr), Thomas Brunet (CNRS and Paris VI University, INSP - 140 rue de Lourmel, 75015 Paris, France, thomas.brunet@insp .jussieu.fr), François Coulouvrat (Institut Jean Le Rond d'Alembert, Université Pierre et Marie Curie, Boites 161 et 162, 4 place Jussieu, 75252 Paris Cedex 05, France, coulouvr@ccr.jussieu.fr)

Acoustical Vortices (AV) are acoustical beams with a phase singularity of screw type. They possess a phase with an helical structure which is winding around the axis of propagation. These twists of phase engender special properties such as the existence of an associated angular momentum and imply a coupling between the axial and transverse components of the beam. Propagation of $\mathrm{AV}$ of finite amplitude follows the classic rule of nonlinear acoustics. Beyond a shock formation distance, AV develop shocks, but the structure of the latters is noticeable. It will be shown by numerical simulations and experimental measurements, that the nonlinear propagation of AV gives birth to 3D shock waves: a classical shock in the direction of propagation plus an azimutal one in the transverse plane. The numerical simulation, based on an original algorithm solving the 3D Khokhlov-Zabolotskaya equation, is used to investigate the dynamic of the formation of the azimutal shock. Experimental observations made with ultrasonics in water confirm the existence of particular shock waves. Finally, a brief discussion of the potential applications will be proposed.

\section{2:40}

3pPAb3. Water flow generation owing to interaction between pulsing bubble and rigid wall. Alexey P. Drozhzhin (Lavrentyev Institute of Hydrodynamics SB RAS, Lavrentyev ave., 15, 630090 Novosibirsk, Russian Federation, ruslan@hydro.nsc.ru), Vyacheslav S. Teslenko (Lavrentyev Institute of Hydrodynamics SB RAS, Lavrentyev ave., 15, 630090 Novosibirsk, Russian Federation, vteslenko@academ.org)

Generating a directed water flow in periodic forming a non-symmetrical acoustic field was experimentally investigated in the work. Periodic acoustic wave field was formed with the help of a vapor-gas bubble pulsing in the hole of a thin diaphragm near a rigid wall. Alternate formation of two water flows moving in opposite directions was found. During the bubble growth stage the flow at velocity V1 was formed in the opposite direction of the plate, and during the bubble collapse stage the flow at velocity V2 was formed towards the plate. By changing the parameter $k=R / r$ where $\mathrm{R}$ was a distance from the hole center to the rigid wall, and $\mathrm{r}$ was a hole radius, the possibility to control average values $\mathrm{V} 1$ and $\mathrm{V} 2$ was shown. It was found that at $\mathrm{k}=\mathrm{R} / \mathrm{r}=5.8 \mathrm{~V} 1=\mathrm{V} 2$, at $\mathrm{k}>5.8 \mathrm{~V} 1>\mathrm{V} 2$, and at $\mathrm{k}<5.8 \mathrm{~V} 1$ was less than V2. It was shown that due to variation of the distance between a pulsing bubble and a rigid wall it was possible to control velocity $\mathrm{V}$ and direction of the total flow of water through the hole. The maximum value of velocity $\mathrm{V}$ reached $40 \mathrm{~cm} / \mathrm{s}$ in the experiment.

\section{3:00}

3pPAb4. Nonlinear ultrasound fields simulation of harmonics from exponential and bessel beams sources. Hicham Jakjoud (Lab. Electrical Systems and Telecommunications, Faculté des Sciences et Techniques Gueliz B.P. 549, 40000 Marrakech, Morocco, hicham.jakjoud@gmail .com), Ahmed Chitnalah (Lab. Electrical Systems and Telecommunications, Faculté des Sciences et Techniques Gueliz B.P. 549, 40000 Marrakech, Morocco, chitnalah@fstg-marrakech.ac.ma), Noureddine Aouzale (Lab. Electrical Systems and Telecommunications, Faculté des Sciences et Techniques Gueliz B.P. 549, 40000 Marrakech, Morocco, aouzale.nour@gmail.com), Djilali Kourtiche (Lab. d'Instrumentation, Electronique de Nancy, UHP Nancy I, BP 239, 54506 Vandouvre, 54506 Nancy, France, djilali.kourtiche@lien.uhp-nancy.fr)

It's well known that the harmonic imaging quality can be improved by using sources that radiate narrower and attenuated sidelobe beams. Hence we try to enhance the harmonics' cartography by studying different source's power distributions. We developed a numerical code, using the spectral method, in order to resolve the parabolic wave equation. The numerical results are compared to those given by other researchers in order to validate our algorithm. Two source's power distributions (exponential and Bessel beams) are studied and compared to the uniform case. The use of exponential source leads to harmonics diagrams without sidelobes neither nearfield oscillations. But the beam width is increasing with propagation. The Bessel 
source presents a limited diffraction beam. The beam width is almost constant throughout the nearfield and the transition zone. The sidelobes have a weak level in the fundamental curves and they don't appear in the second harmonic ones.

\section{3:20}

3pPAb5. Experimental confirmation of the theory of acoustic radiation pressure applying on transparent interfaces. Bruno Issenmann (Bordeaux University - $\mathrm{CPMOH,} 351$ cours de la Liberation, 33405 Talence Cedex, France, b.issenmann@cpmoh.u-bordeaux1.fr), Alice Nicolas (Laboratoire de Physique Matiere Condensee - Univ. Nice, Parc Valrose - 28, avenue Valrose, 06108 Nice Cedex 02, France, Alice .NICOLAS@unice.fr), Regis Wunenburger (Bordeaux University - CPMOH, 351 cours de la Liberation, 33405 Talence Cedex, France, r.wunenburger@cpmoh.u-bordeaux1.fr), Sébastien_Manneville (Ecole
Normale Supérieure de Lyon, 46 allée d'Italie, 69364 Lyon Cedex 07, France, sebastien.manneville@ens-lyon.fr), Jean-Pierre Delville (Bordeaux University - CPMOH, 351 cours de la Liberation, 33405 Talence Cedex, France, jp.delville@cpmoh.u-bordeaux1.fr)

Since Rayleigh and Brilloin, the acoustic radiation pressure has been the subject of several theoretical works, but of few quantitative tests. Whereas the radiation pressure acting on perfectly reflecting or perfectly absorbing solid targets is commonly used for the calibration of high intensity focused ultrasonic beams, it has never been quantitatively studied on acoustically transparent interfaces. Using an acoustically transparent liquid-liquid interface deformed by the radiation pressure of a focused, continuous wave beam, we have tested the theory of the acoustic radiation pressure acting on transparent interfaces for the first time. At large intensity, depending on the direction of propagation of the beam, we observe surprising interface shapes such as drop emitting jets and "nipple-like" deformations.

WEDNESDAY AFTERNOON, 2 JULY 2008

ROOM 241, 2:00 TO 3:40 P.M.

\title{
Session 3pPPa
}

\section{Psychological and Physiological Acoustics: General Topics in Psychological and Physiological Acoustics II}

\author{
Elizabeth Strickland, Cochair \\ Purdue University, 500 Oval Drive, West Lafayette, IN 47907, USA
}

Armin Kohlrausch, Cochair

Philips Research Europe, Digital Signal Processing (MS WO02), High Tech Campus 36, Eindhoven, 5656 AE, Netherlands

\author{
Alain De Cheveigne, Cochair \\ CNRS, Universite Paris 5, Ecole Normale Superieure, 29 rue d'Ulm, Paris, 75230, France
}

\section{Contributed Papers}

\section{2:00}

3pPPa1. A pair of spectral notches which plays a role as a spectral cue in the vertical localization, and it's application to estimation of sound source elevation from binaural signals. Kazuhiro Iida (Chiba Institute of Technology, 2-17-1 Tsudanuma, 275-0016 Narashino, Japan, kazuhiro.iida @it-chiba.ac.jp)

The author [Applied Acoustics, 68, 835-850 (2007)] proposed a parametric HRTF model for vertical sound localization. The parametric HRTF is recomposed only of the spectral peaks and notches extracted from the measured HRTF. The results of the median plane localization tests, which were carried out using the parametric HRTFs with various combinations of spectral peaks and notches, show that the pair of first and second notches (N1 and N2) above $5 \mathrm{kHz}$ can be regarded as spectral cues. In this research, estimation of the elevation of sound source in the upper median plane by extracting $\mathrm{N} 1$ and $\mathrm{N} 2$ frequencies from binaural input signal was carried out. The kinds of sound sources were female voice, male voice, music, white noise, and pink noise. The results show that the estimation is accurate for almost of all the elevation and of all the kind of sound sources.

\section{2:20}

3pPPa2. Trading of intensity and interaural correlation cues in the perception of loudness. John F. Culling (Cardiff University, School of Psychology, Tower Building, Park Place, CF10 3AT Cardiff, UK, CullingJ@cardiff.ac.uk), Hannah G. Lewis (Cardiff University, School of
Psychology, Tower Building, Park Place, CF10 3AT Cardiff, UK, lewishg @Cardiff.ac.uk)

When a signal is added to noise in the $\operatorname{NoS} \pi$ binaural configuration, a reduction in interaural cross-correlation (IACC) occurs at the signal frequency. Increases in tone intensity produce decreases in IACC. Consistent with this relationship, direct manipulations of IACC can result in the perception of an added signal and progressive reduction in IACC produces progressive increases in the loudness of this signal [Culling et al. $2001 \mathrm{~J}$. Acoust. Soc. Am. 110, 1020-1029]. In the present study, a narrow sub-band of noise $(460-540 \mathrm{~Hz})$ embedded within a broadband $(0-3 \mathrm{kHz})$ diotic noise was manipulated in both intensity and IACC in a 3-interval, odd-one-out task. In the reference intervals, IACC was zero and the spectrum was flat. In the target interval, both the IACC and the intensity of the target band were incremented. These increases have opposing effects on loudness. Correct identification of the target interval followed a U-shape as a function of the size of intensity increment. The minimum of the function was at chance performance, indicating that the opposing cues were fully traded.

\section{2:40}

3pPPa3. A comparison between interaural level difference and interaural correlation uncertainty on binaural signal detection thresholds. Nicolas Le Goff (Technical University Eindhoven, Den Dolech 2, 5600 MB Eindhoven, Netherlands, n.legoff@tm.tue.nl), Armin Kohlrausch (Philips Research Europe, Digital Signal Processing (MS WO02), High Tech Campus 36, 5656 AE Eindhoven, Netherlands, 
armin.kohlrausch@philips.com),Jeroen Breebaart(Philips Research Europe, Digital Signal Processing (MS WO02), High Tech Campus 36, 5656 AE Eindhoven, Netherlands, jeroen.breebaart@philips.com)

Experiments were performed to study the effect of the presence of interaural level differences (ILDs) on binaural detection abilities. The subjects had to detect an interaurally out-of-phase $500-\mathrm{Hz}$ tone in the presence of a diotic noise masker that had a bandwidth of either $1 \mathrm{kHz}, 100 \mathrm{~Hz}$ or $10 \mathrm{~Hz}$. Experiments were conducted for both frozen and running-noise conditions and ILDs up to $30 \mathrm{~dB}$ were applied to both signal and masker. The results indicate an ILD dependency that varies with the bandwidth of the masker Furthermore, with increasing ILDs, the difference in detection thresholds between frozen and running-noise conditions was larger for narrow-band noise conditions. Similar observations were made by previous investigations on the influence of reduced masker correlation. Both data sets are compared in order to find possible similarities in the mechanisms involved in the detection tasks.

3:00

3pPPa4. The effect of a speech target's motion on its recognition in the presence of simultaneous competing sentences. Wesley Grantham (Vanderbilt Bill Wilkerson Center, Department of Hearing and Speech Sciences, 1215 21st Avenue South, Room 8310, Nashville, TN 37232-8242, USA, d.wesley.grantham@vanderbilt.edu), Todd Ricketts (Vanderbilt Bill Wilkerson Center, Department of Hearing and Speech Sciences, 1215 21st Avenue South, Room 8310, Nashville, TN 37232-8242, USA, todd.a.ricketts@vanderbilt.edu), Daniel Ashmead (Vanderbilt Bill Wilkerson Center, Department of Hearing and Speech Sciences, 1215 21st Avenue South, Room 8310, Nashville, TN 37232-8242, USA, daniel.h.ashmead@vanderbilt.edu)

Prior knowledge of where to listen significantly improves speech recognition of target sentences presented in the presence of distracter sentences coming from different locations [G. Kidd et al., J. Acoust. Soc. Am. 118, 3804-3815 (2005)]. The present study extended the work of Kidd et al. by measuring the effect of a target's motion on its recognition when competing messages are present. In an anechoic chamber normal-hearing subjects were presented with three simultaneous sentences from the CRM corpus and were instructed to indicate key words from the target sentence (identified by a call-sign previously known to the subject). In the stationary condition the three sentences came from $-60^{\circ}, 0^{\circ}$, and $+60^{\circ}$ azimuth. In the moving condition, the target source moved during its on-time (e.g., from $-60^{\circ}$ to $0^{\circ}$ ) while the two distracter sentences were stationary (e.g., at $\pm 60^{\circ}$ ). In both cases, subjects either knew in advance where the target would be (Certain Condition) or did not know (Uncertain Condition). It is hypothesized that motion of the target will result in a release from informational masking. That is, the detrimental effect of location uncertainty observed with the stationary targets will be reduced or eliminated when the target is moving.

\section{$3: 20$}

3pPPa5. Frequency selectivity in diotic and dichotic masking conditions for normal-hearing and hearing-impaired listeners. Marc Nitschmann (Universität Oldenburg, Medizinische Physik, Carl-vonOssietzky Str. 9-11, 26111 Oldenburg, Germany, marc.nitschmann @ uni-oldenburg.de), Jesko Verhey (Universität Oldenburg, Medizinische Physik, Carl-von-Ossietzky Str. 9-11, 26111 Oldenburg, Germany, jesko.verhey@uni-oldenburg.de), Birger Kollmeier (Universität Oldenburg, Medizinische Physik, Carl-von-Ossietzky Str. 9-11, 26111 Oldenburg, Germany, birger.kollmeier@uni-oldenburg.de)

Previous studies argued that, for broadband maskers, the operational binaural critical bandwidth is similar to the monaural critical bandwidth. The aim of the present study was to test this hypothesis using the notched noise paradigm. Thresholds were measured for a diotic masker and a signal that was either in phase or had an interaural phase difference of 180 degrees. For comparison, thresholds were also measured for a broadband masker (without a notch) at various masker levels to account for possible level effects of the binaural masking level difference (BMLD). Normal-hearing and hard-of-hearing listeners with various degrees of sensorineural hearing impairment participated in the experiment. For all subjects, the thresholds decreased faster for the diotic than the dichotic signal as the notch width increased. The comparison with the data for the broadband masker indicated that this decrease of the BMLD is not due to the decrease of masker level within the auditory filter centered at the signal frequency. The thresholds can be predicted by an equalization-cancellation model assuming a slightly broader auditory filter in the dichotic masking condition. A possible realization of this different binaural auditory filter shape is adding to the onfrequency filter fractions of the adjacent filters. 


\title{
Session 3pPPb
}

\section{Psychological and Physiological Acoustics: General Topics in Psychological and Physiological Acoustics III}

\author{
Elizabeth Strickland, Cochair \\ Purdue University, 500 Oval Drive, West Lafayette, IN 47907, USA
}

Armin Kohlrausch, Cochair

Philips Research Europe, Digital Signal Processing (MS WO02), High Tech Campus 36, Eindhoven, 5656 AE, Netherlands

Alain De Cheveigne, Cochair

CNRS, Universite Paris 5, Ecole Normale Superieure, 29 rue d'Ulm, Paris, 75230, France

\section{Contributed Papers}

\section{2:00}

3pPPb1. Effects on cochlear frequency selectivity after hypobaric pressure exposure. Jonas Brännström (Department of Audiology, Malmö University Hospital and Lund University, 20505 Malmö, Sweden, jonas.brannstrom@skane.se), Jan Grenner (Department of Audiology, Lund University Hospital, 22185 Lund, Sweden, jan.grenner@skane.se)

The effects of hypobaric pressure chamber exposure was measured in noise in ten patients with monaural fluctuating low-frequency hearing loss (FLFHL) such as Ménière's disease using psychophysical tuning curves (PTC), transiently evoked otoacoustic emissions (TEOAE), binaural pitch matches and speech recognition scores (SRS) in noise. In the literature, reversible hearing losses have been observed in about $50 \%$ of the patients, but sometimes improved SRS can be observed in patients without hearing threshold improvement. This indicates possible effects of pressure treatment on cochlear frequency selectivity. The relative overpressure in the middle ear obtained after repeated exposures in hypobaric pressure chamber (total duration 18.5 to 28 minutes) was used to impose pressure gradients to the inner ear. The results indicated that the treatment effects were small, but slightly improved SRS in noise, TEOAEs emission strength and PTCs were observed after treatment. Pure tone hearing thresholds improved only for patients exposed to longer treatment durations. Subjective improvement at follow-up could not be predicted from the results. Although the effects were small, the data suggest that hypobaric pressure treatment may improve cochlear frequency selectivity in the affected ear in patients with monaural FLFHL.

\section{2:20}

3pPPb2. IPods listening levels on London Underground. Stephen Dance (London South Bank University, FESBE, Borough Road, SE1 0AA London, UK, dances@1sbu.ac.uk), Phil Wash (Bickerdike Allen Partners, 117-121 Salusbury Road, Queens Park, NW6 6RG London, UK, pwash @bickerdikeallen.com)

In the last 5 years the prevalence of the iPod/MP3 players has grown exponentially. The use of such in-earphones under urban conditions has been reported widely in the press at the anecdotal level. This study compared listening levels under quiet conditions and that representative of a London Underground train journey. Calibrated recordings of underground trains running in tunnels were played through loudspeakers in an anechoic chamber, whilst pop music or speech podcasts were played through the iBuds. Thirty-three participates listened to the iPod whilst a Binaural Head and Torso measured the noise levels through the in-earphones. The participants had time to adjust the volume setting on each occasion. A small audiometric study was undertaken for those participates with particularly high and low volume settings a week after the tests were completed. Results show very high volume settings were used when speech was played with the train noise.

\section{2:40}

3pPPb3. Assessment of hearing damage when listening to music through a personal digital audio player. Adriano Farina (Liceo Classico G.D. Romagnosi, Viale Maria Luigia 1, 43100 Parma, Italy, adriano @ adrianofarina.it)

This study evaluates the sound pressure at the ears of users of personal digital audio players, equipped with in-ear earbuds. More than 50 devices were measured, provided by students aged 15/18. EN standard 50332 specifies the techniques for measuring the sound pressure level at the ears employing an head and torso simulator equipped with binaural microphones, when the gain control of the device is set to maximum. In this study, however, two measurmenets were performed on each device, one at maximum level, and another at the level at which the device had been left by the user after last listening. This way, on a statistical basis, it is possible to relate the effective exposure in daily usage with the maximum loudness of which the device is capable. Furthermore the study did also analyze the spectrum of the test signal: whilst the EN standard mandates for the IEC programme simulation noise, the analysis of thousands songs taken from the players under test revealed a slightly different spectrum, characterized by more boost at low frequencies and larger crest factor. The results of the study did show a relevant hearing risk for users of these personal digital audio players, which exceed noise exposure limits even when used for just one hour per day.

\section{3:00}

3pPPb4. The Healthy Benefits of Isolating Earphones. Jeremie Voix (Sonomax Hearing Healthcare Inc, 8375 Mayrand, Montreal, QC H4P 2E2, Canada, jvoix@sonomax.com), Cecile Le Cocq (Ecole de Technologie Superieure, 1100 Notre-Dame Ouest, Montreal, QC H4P 2E2, Canada, clecocq@mec.etsmtl.ca), Lee D. Hager (Sonomax Hearing Healthcare Inc, 8375 Mayrand, Montreal, QC H4P 2E2, Canada, lhager@sonomax.com)

With the ubiquitous presence of Personal Stereo Players (PSPs), namely iPods $^{\mathrm{TM}}$ and the like, many hearing conservationists have raised concerns about the temporary and permanent hearing damages that could result from long exposure to loud music playback, especially among adolescents and teenagers. The crux of the problem can be identified as an overexposure of the auditory system. In order to reduce the dose received by the PSP listener, the playback level and/or the duration should be reduced. Assuming that the duration of the music playback experience is really up to the user, the remaining parameter is the music playback level. The purpose of this study is to first understand - from the available public and scientific literature - what factors are influencing the PSP playback level; and second, to investigate if the use of earphones featuring good attenuation of the ambient noise level would lead to a reduced playback level, hence a reduced dose and eventually less auditory damage. Other benefits on sound quality that are associated with isolating earphones will be presented in a third part. Finally the article will review other safety mechanisms that could be used in earphones and PSP to make them safe for the hearing. 
3:20

3pPPb5. Potentiation of noise-induced hearing loss by aviation fuel exposure in rats. Laurence D. Fechter (Loma Linda VA Medical Center, Research Service (151), 11201 Benton Street, Loma Linda, CA 92357, USA, larry.fechter@va.gov), Caroline A. Gearhart (Loma Linda VA Medical Center, Research Service (151), 11201 Benton Street, Loma Linda, CA 92357, USA, caroline.gearhart@va.gov), Sherry Fulton (Loma Linda VA Medical Center, Research Service (151), 11201 Benton Street, Loma Linda, CA 92357, USA, sherry.fulton@va.gov), Yoon Hwan Kim (Dept Otolaryngology Cheongju St Mary's Hospital, 589-5 JuJung-Dong, SangDang-Gu, Cheong-Ju, 360-568 Chung-Buk, Republic of Korea, akeell@hanmail.net)

Clear standards have been established for workplace noise. These are designed to protect workers against noise-induced hearing loss. That occupa- tional hearing loss still occurs despite such standards may reflect a variety of issues ranging from enforcement of standards, to adequacy of standards, and problems of noise assessment. An additional factor that influences susceptibility to noise-induced hearing loss is the presence of chemical contaminants in the workplace that render the auditory system especially vulnerable to noise. We report on the ability of aviation fuels to potentiate noise-induced hearing loss in laboratory rats. Subjects were exposed to JP-8 jet fuel, a synthetic fuel produced from coal using the Fischer-Tropsch method, or to clean air. Half of the subjects received a moderate noise exposure following fuel or air exposure. The noise exposure was designed to produce a temporary threshold shift. JP-8 + noise produced greater disruption of distortion product otoacoustic emissions as well as the auditory threshold than did noise alone. Supported by VA Rehabilitation Service grants and the American Petroleum Institute.

WEDNESDAY AFTERNOON, 2 JULY 2008

ROOM 240, 2:00 TO 3:40 P.M.

\title{
Session 3pSCa
}

\section{Speech Communication: Phonetics}

\author{
Joseph S. Perkell, Chair \\ Massachusetts Institute of Technology, Research Laboratory of Electronics, Room 36-511, 50 Vassar St., Cambridge, MA \\ 02139-4307, USA
}

\section{Contributed Papers}

\section{2:00}

3pSCa1. Auditory and somatosensory goals for sibilants. Joseph S. Perkell (Massachusetts Institute of Technology, Research Laboratory of Electronics, Room 36-511, 50 Vassar St., Cambridge, MA 02139-4307, USA, perkell@mit.edu), Melanie L. Matthies (Massachusetts Institute of Technology, Research Laboratory of Electronics, Room 36-511, 50 Vassar St., Cambridge, MA 02139-4307, USA, matthies@bu.edu), Satrajit S. Ghosh (Massachusetts Institute of Technology, Research Laboratory of Electronics, Room 36-511, 50 Vassar St., Cambridge, MA 02139-4307, USA, satra@speech.mit.edu), Edwin Maas (Massachusetts Institute of Technology, Research Laboratory of Electronics, Room 36-511, 50 Vassar St., Cambridge, MA 02139-4307, USA, maas@bu.edu), Alexandra Hanson (Massachusetts Institute of Technology, Research Laboratory of Electronics, Room 36-511, 50 Vassar St., Cambridge, MA 02139-4307, USA, alex.hanson@gmail.com), Frank H. Guenther (Boston University, 677 Beacon Street, Boston, MA 02215, USA, guenther@cns .bu.edu), Harlan Lane (Massachusetts Institute of Technology, Research Laboratory of Electronics, Room 36-511, 50 Vassar St., Cambridge, MA 02139-4307, USA, lane@ speech.mit.edu), Lucie Ménard (Département de linguistique et de didactique des langues, Université du Québec à Montréal, Case postale 8888, succursale Centre-ville, Montréal, QC H3C 3P8, Canada, menard.lucie@uqam.ca), Mark Tiede (Massachusetts Institute of Technology, Research Laboratory of Electronics, Room 36-511, 50 Vassar St., Cambridge, MA 02139-4307, USA, tiede@ speech.mit.edu)

In prior studies [JSLHR 47, 1259-69, 2004; JASA 116, 2338-44, 2004], we found that speakers' auditory acuity for synthetic sibilant $(/ \mathrm{s} /, / \mathrm{sh} /)$ and vowel stimuli was correlated with the degree of acoustic contrast they produced. This outcome is consistent with the view that the articulatory movements underlying phonemic contrasts have auditory goals. There was also a cross-speaker relation between sibilant contrast and use of contact between the tongue tip and lower alveolar ridge for $/ \mathrm{s} /$ (but not /sh), indicating that such contact is a somatosensory goal for /s/. In the current study, another, larger group of subjects also showed relations between measures of auditory acuity and acoustic contrast for sibilants and vowels. To determine whether the idea of a somatosensory goal for /s/ would also be supported by a relation between acuity and contrast, we made measurements of somatosensory discrimination. Small plastic (JVP) domes with grooves of differ- ent spacing were pressed against each subject's tongue tip and the subject was asked to identify one of four possible orientations of the grooves. A wide range of individual performance was observed with a bias-corrected sensitivity measure. Correlations with produced sibilant contrast and further analyses will be reported. [Research supported by NIDCD, NIH.]

\section{2:20}

3pSCa2. Perceptual explanations of articulatory variability in the realisation of the nasal feature for the consonants. Jacqueline Vaissière (Laboratory of Phonetics and Phonology, 19 rue des bernardins, 75005 Paris, France, jacqueline.vaissiere@univ-paris3.fr)

This paper illustrates how the perceptual constraints explain the observed articulatory and aerodynamic inter-speakers and inter-languages variability between initial nasal consonants $(/ \mathrm{n} /)$, and unreleased final consonants $(/ \mathrm{N} /)$. In the case of $/ \mathrm{n} /$, only a short, well-defined region around the consonant release needs to be nasalized for the consonant to be perceived as nasal. In the case of /N/, because of the lack of a nasal release, a longer span is needed for deciphering the presence of nasalisation; contextual nasalization of the preceding vowel can be avoided, but in that case, the nasal murmur has to be long to be perceived (since the first part of the murmur is perceptually masked by the vowel), and/or /N/ has to be released. Such observations argue for the division of the nasal consonant into three parts, onset, murmur and release: the realisation of the nasal feature is aligned around the $/ \mathrm{N} /$ onset, and around the $/ \mathrm{n} /$ offset. It also argues for a better integration of the perceptual requirements and masking phenomena in modelling the observed variability. Finally, it shows that a feature can be realized very differently depending on the position of the phoneme in the syllable.

\section{2:40}

3pSCa3. Final consonant voicing and vowel height contrasts in whispered speech. Yana D Gilichinskaya (City University of New York, Graduate Center, 365 Fifth Avenue, New York, NY 10016, USA, ygilichinskaya@gc.cuny.edu), Winifred Strange (CUNY Graduate Center, 
365 5th Avenue, Program in Speech-Language-Hearing Sciences, New York, NY 10016, USA, strangepin@aol.com)

Whispered speech is a naturally distorted speech signal. Whereas it preserves some characteristics of fully phonated speech, some important acoustic cues are removed, diminished or altered. The prominence of acoustic cues in whispered speech may change due to the physical properties of the whispered speech signal, i.e., decreased intensity, the absence of periodic vibration of the vocal folds, damping of F1, shift of the formants and flattening of the amplitude envelope. Such changes affect the acoustic cues both for vowels (e.g. vowel height) and consonants (e.g. voicing contrasts). The objective of the present project was to explore the acoustic cues for poststressed syllable-final consonant voicing contrasts and the vowels preceding them in continuous whispered speech of American English speakers and to compare the results with those in fully phonated speech. The stimuli were recorded in the carrier sentence "I'll utter /habVC/ off the list". The consonant pairs included voiced/voiceless bilabial stops /b-p/ and labiodental fricatives /f-v/, each combined with $11 \mathrm{AE}$ vowels /i, I, "epsilon", e, æ, a, "turned-v", o, "open-o", u, "horseshoe"l. Preliminary results showed that vowels had longer duration in whispered speech than in fully phonated speech. Spectral dispersion, temporal contrastivness of vowels; F1, and vowel duration cues will be reported in the presentation.

\section{3:00}

3pSCa4. Production and perception of vietnamese short vowels Nguyen Viet Son (MICA center HUT - CNRS/UMI2954 INP Grenoble, C10 Hanoi university of Technology, No1 Dai Co Viet street - Hai Ba Trung, 0084 Hanoi, Viet Nam, viet-son.nguyen@mica.edu.vn), René Carré (Laboratoire Dynamique du Langage, UMR 5596, CNRS, Université Lyon 2, 14 Avenue Marcelin Berthelot, 69363 Lyon cedex 07, France, recarre@wanadoo.fr), Eric Castelli (MICA center HUT - CNRS/UMI2954 INP Grenoble, C10 Hanoi university of Technology, No1 Dai Co Viet street - Hai Ba Trung, 0084 Hanoi, Viet Nam, Eric.Castelli@mica.edu.vn)

It is well known that vowels can be produced in isolation, acoustically stable in such a way that they are represented as points in the F1-F2-F3 space. Vietnamese language presents 13 vowels, however, Vietnamese can only pronounce 9 vowels $[\mathrm{i}, \mathrm{u}, \mathrm{e}, \mathrm{o}, \omega, \mathrm{a}, \sigma, \varepsilon, \gamma]$ in isolated mode. A pre- vious study showed that the 4 remaining vowels $[\alpha, \delta, \nu, \epsilon]$ have the same acoustic characteristics $(\mathrm{F} 1, \mathrm{~F} 2, \mathrm{~F} 3)$ as, respectively, the vowels [a, $\varepsilon, \sigma, \gamma]$, but their dynamic characteristics (the rate of $\mathrm{CV}$ transitions) are clearly distinct. Measurements show us that vowels durations of $[\alpha, \nu]$ are always shorter than the one of corresponding classical vowels $[a, \gamma]$ and are not acoustically stable. For test perceptions (with 10 Vietnamese people), synthesized syllables $[\mathrm{a}-\mathrm{t}]$ and $[\gamma-\mathrm{t}]$ with changing vowel duration are recognized as $[\alpha-\mathrm{t}, \nu-\mathrm{t}]$ when then duration of initial vowel $[\mathrm{a}, \gamma]$ are $60 \%-70 \%$ shorter. It means that the vowel duration is an important parameter that allows Vietnamese distinguishing the long vowels and short vowels in Vietnamese language. This paper further analyses the production of Vietnamese $\mathrm{CV}$, including long vowels and short vowels in terms of duration, formant evolution and rate of C.V transitions.

\section{3:20}

3pSCa5. Acoustic properties of the interdental approximant in Kagayanen. Kenneth S. Olson (SIL International, 7500 W Camp Wisdom Rd, Dallas, TX 75236, USA, Ken_Olson@sil.org), Jeff Mielke (University of Ottawa, Arts Hall, 70 Laurier Ave East, Room 401, Ottawa, ON K1N 6N5, Canada, jmielke@uOttawa.ca)

This paper describes the acoustic properties of the interdental approximant, a rare speech sound reported to date only in a dozen languages in the Philippines. The measurements analyzed here are based on recordings of one female speaker of Kagayanen. The interdental approximant exhibits acoustic characteristics typical of semi-vowels: the formant pattern is similar to that of vowels, and the formant transitions with adjoining vowels have a long duration, usually $35 \mathrm{~ms}$ or more. The values of F2 and F3 are analogous to those of the Kagayanen [1], a prototypical voiced alveolar lateral; the only significant formant value difference between the two sounds involves F1: the interdental approximant has a mean of $508 \mathrm{~Hz}(\mathrm{n}=9)$, whereas the mean for [1] is $368 \mathrm{~Hz}(\mathrm{n}=9)$. On the other hand, while these two segments sound impressionistically similar, the interdental approximant differs acoustically from [1] in several other respects as well: there is no abrupt change in the formant pattern at the junctures with adjoining vowels, the higher formants (F6 and above) are not reduced in intensity, and F3 is not enhanced in intensity compared to semi-vowels.

WEDNESDAY AFTERNOON, 2 JULY 2008

ROOM 250B, 2:00 TO 3:40 P.M.

\title{
Session 3pSCb
}

\section{Speech Communication: Prosody II}

\author{
Marion Dohen, Chair \\ Speech and Cognition Department, GIPSA-lab, 46 avenue Félix Viallet, Grenoble, 38031, France
}

\section{Contributed Papers}

\section{2:00}

3pSCb1. Audiovisual perception of prosodic contrastive focus in whispered French. Marion Dohen (Speech and Cognition Department, GIPSA-lab, 46 avenue Félix Viallet, 38031 Grenoble, France, marion.dohen@gipsa-lab.inpg.fr), Hélène Loevenbruck (Speech and Cognition Department, GIPSA-lab, 46 avenue Félix Viallet, 38031 Grenoble, France, Helene.Loevenbruck@gipsa-lab.inpg.fr)

This study investigates the potential contribution of visual information in the audiovisual perception of prosodic contrastive focus in French. Contrastive focus is used to attract the listener's attention to a specific part of the utterance. Mostly conceived of as auditory/acoustic, it also has visible correlates which have been shown to be perceived. This study aimed at analyzing the interaction between audition and vision for the perception of prosodic focus by using a whispered speech paradigm. It was based on audiovisual recordings from 4 speakers wearing no facial markers. It com- bined performance assessment to reaction time measurements and confirmed and extended preliminary results obtained on 2 speakers wearing facial markers (needed for a parallel articulatory analysis). The results showed that adding vision to audition for the perception of prosodic focus can not only improve perception performances but can also reduce perceptual cognitive load. A further analysis suggested that the two modalities are integrated for the perception of prosodic focus. Visual only perception was facilitated for whispered speech suggesting an enhancement of visual cues. Moreover, the absence of facial markers did not impair perception. Therefore facial markers do not seem to enhance the relative importance/salience of visual cues.

\section{2:20}

3pSCb2. Effects of boundary tones on accent-related F0 peak alignment. Yen-Liang Shue (Univ. of California Los Angeles, Dept. of Electrical Engineering, 405 Hilgard Ave., Los Angeles, CA 90095, USA, 
yshue@ee.ucla.edu),Markus Iseli(Univ. of California Los Angeles, Dept. of Electrical Engineering, 405 Hilgard Ave., Los Angeles, CA 90095, USA, iseli@ee.ucla.edu), Stefanie Shattuck-Hufnagel (Massachusetts Institute of Technology, Research Laboratory of Electronics, Speech Communication Group, 77 Massachusetts Ave., Cambridge, MA 02139, USA, stef@speech.mit.edu), Nanette Veilleux (Simmons College, Dept. of Computer Science, 300 The Fenway, Boston, MA 02115, USA, nanette.veilleux@simmons.edu), Sun-Ah Jun (Univ. of California Los Angeles, Dept. of Linguistics, 405 Hilgard Ave., Los Angeles, CA 90095, USA, jun@humnet.ucla.edu), Abeer Alwan (University of California, 405 Hilgard Ave, Los Angeles, CA 90095, USA, alwan@ee.ucla.edu)

Speakers sometimes delay the F0 peak of a high accent beyond the accented syllable (Silverman and Pierrehumbert, Papers in Laboratory Phonology I, 1990; Xu, Phonetica, 2001). Previous studies of factors affecting peak alignment focused on the phrasal position of the accented word and the segments of the accented syllable (Jilka and Möbius, Interspeech 2007). Shue et al. (Interspeech 2007) hypothesized that if an accented word also contains boundary tones, that could influence the position and height of the F0 peak. A test corpus controlling for vowel type, syllable number and position of the focused word, based on the utterances "Dagada gave Anne a dada (daily)" and "A dada gave Anne dagadas (daily)", was produced using four prosodically different contours, involving high $\left(\mathrm{H}^{*}\right)$ and low $\left(\mathrm{L}^{*}\right)$ pitch accents on either "dagada" or "dada", in declarative and interrogative forms eliciting different boundary tones. Analysis of five repetitions spoken by four male and four female native American English speakers reveals that for most speakers, the presence of boundary tones on the target word shifts the accentrelated F0 peak earlier when other factors are held constant. Preliminary results suggest effects of boundary tone on $\mathrm{H}^{*} \mathrm{~F} 0$ peak height as well.

\section{2:40}

3pSCb3. Double focus in general American English. Fang Liu (The University of Chicago, Department of Linguistics, 1010 E. 59th Street, Chicago, IL 60637, USA, liufang@uchicago.edu)

This study investigates the acoustic realization of double focus in statements and declarative questions in English. Five speakers produced eight sets of utterances with alternating focus (medial, final, or double) and sentence type (statement or declarative question) conditions. F0 analyses indicate that the effect of double focus on the global pitch range and the local pitch target is somewhat moderate when compared to that of single focus: 1) Post-focus pitch range suppression shows a smaller magnitude in doublethan in medial-focused statements, 2) Post-focus pitch range raising is less dramatic in double- than in medial-focused questions, and it ends before the stressed syllable of the second focus in double-focused questions, in order to realize a rising pitch target for the on-focus stressed syllable, and 3) pitch targets of on-focus stressed syllables are more fully realized (i.e., showing steeper slopes or higher registers) in single- than in double-focused sentences. Duration analyses indicate that the length of the double-focused item is in between those of the corresponding single- and non-focused item, with the single-focused stressed syllable having the longest duration. These findings demonstrate a delicate balance among the realization of lexical stress, focus, and sentence type in English.

\section{3:00}

3pSCb4. Melodic prominences structures: exploring to what extent the speaker variability is spreading. Geneviève Caelen-Haumont (MICA Center, C10, Hanoi Universirty of Technology, 1 Dai Co Viet Str., Hai Ba Trung, Hanoi, Hanoi, Viet Nam, genevieve.caelen@mica.edu.vn)

Melodic prominences in speech have a deserved reputation of conveying a great part of variability, as they are greatly based on subjective impulse and feelings. We think that these specific contours are nevertheless relevant, and that their structure, while obeying to internal laws of regulation, allow also a range of speaker variability. Generally no specific tool was used to describe these FO contours with precision. An automatic analysis tool MELISM was then developed by Caelen-Haumont and Auran (2004) allowing accurate descriptions of F0 salience (melisms). This paper aims at $1^{\circ}$ describing the MELISM tool, the automatic segmentation, the annotation, the melodic labelling of the melism structure it provides in the prosody domain $2^{\circ}$ presenting the main results over 4 speaker exploring to what extent the speaker variability spreads. The melism structures are explored and analysed such as the whole variability is reduced through three major components (onset, nucleus, coda) and their respective subparts, where only the nucleus is the compulsory part. Statistics running on these structures put to light a set of interesting laws about the internal regulation of melisms but in the other side, the part devoted to the speaker variability.

\section{3:20}

3pSCb5. How prosody correlates with syntax: An observation on Sakizaya, an endangered Formosan language. Sally Chen (Grad. Inst. of Linguistics, National Taiwan Univ., 1, Roosevelt Rd. Sec. 4, 106 Taipei, Taiwan, d93142002@ntu.edu.tw), Wen-Chi Shen (Grad. Inst. of Linguistics, National Taiwan Univ., 1, Roosevelt Rd. Sec. 4, 106 Taipei, Taiwan, r94142007@ntu.edu.tw), Li-May Sung (Grad. Inst. of Linguistics, National Taiwan Univ., 1, Roosevelt Rd. Sec. 4, 106 Taipei, Taiwan, limay@ntu.edu.tw), Janice Fon (Grad. Inst. of Linguistics, National Taiwan Univ., 1, Roosevelt Rd. Sec. 4, 106 Taipei, Taiwan, jfon@ntu.edu.tw)

This pioneering study investigates the relationship between prosody and syntax of Sakizaya, an endangered Formosan language in eastern Taiwan. Both elicitation data and spontaneous data were collected from five informants (aged 48 to 74), in order to provide a more thorough sketch on this VSO language with clear case marking and voice systems. Preliminary results showed that in descriptive sentences, prosodic units and syntactic units do correlate with each other, depending on which of the four voices is used. (The four voices in Sakizaya: agent-, patient-, instrumental, and locative voices) However, once specific functions are imposed onto a sentence, they will override this "default" setting, and adopt corresponding prosodic patterns for the ease of communication. For example, in this language, the default position to receive a nuclear accent should be the ultimate syllable of a sentence; however, we observed an accent shift onto the penultimate syllable for yes/no questions, exclamations, as well as continuously progressive sentences. In negation sentences, pitch peaks are distinctively higher in the accented syllable of the negator (which posited sentence-initially), instead of the ultimate syllable in sentence-final position. Lastly, case markers and voice markers were found as preferred positions for speech planning and repair. 


\title{
Session 3pSP
}

\section{Signal Processing in Acoustics, Acoustical Oceanography, and ECUA: Model-Based Signal Processing I}

\author{
Sean Lehman, Cochair \\ Lawrence Livermore Natl. Lab., Livermore, CA 94551, USA \\ Christian Pichot, Cochair \\ Antennas \& Telecommunications Laboratory, University of Nice-Sophia Antipolis, France
}

Invited Papers

\begin{abstract}
2:00
3pSP1. Model-based algorithms for detecting damage in ultrasonic nondestructive evaluation measurements. Grace A. Clark (Lawrence Livermore National Laboratory, 7000 East Ave., L-130, Livermore, CA 94550, USA, clark9@1ln1.gov)

This work addresses the "As-Built" modeling problem in ultrasonic nondestructive evaluation (NDE), in which one is given measurements of a mechanical part before and after use. The condition of the "as-built" part (prior to use) is known, so that after the part has been used, it can be tested for damage. This enables a two-step model-based approach: (1) Given input-output measurements (AScans), estimate a dynamic prediction-error model of the "as-built" measurement(s) using system identification algorithms. The model is validated by testing the innovations (residuals) for statistical whiteness and then stored for future use. (2) Later, when testing the part for damage, the error between the measurement and the output of the stored model is tested against a short-term whiteness confidence interval test statistic. If the part passes the test, this implies that the model remains valid and the part is declared undamaged. If the part fails the test, this indicates a model mismatch, which means that the part's acoustic properties have changed, and the part is declared damaged. Performance of the algorithms is demonstrated using real measurements, receiver operating characteristic (ROC) curves and a confidence interval about the probability of correct classification.
\end{abstract}

$2: 20$

3pSP2. Signal-based ray tracing modeling in complex tectonics. Paul Cristini (CNRS-UMR5212 Modélisation et Imagerie en Géosciences, UPPA BP115, 64013 Pau, France, paul.cristini@ univ-pau.fr), Eric De Bazelaire (11, Route du Bourg, 64230 Beyrie-enBéarn, France, edebaz@wanadoo.fr), Charles Revaux (50, Rue Lagardere, 64000 Pau, France, charles.revaux@wanadoo.fr)

The objective of this talk is to present a novel method which can perform the fast computation of the times of arrival of seismic waves which propagate between a source and an array of receivers in a stratified medium. This method combines signal processing concepts for the approximation of interfaces and wavefronts, and ray theory for the propagation of wavefronts. The main idea is to put in adequation the computations with the the precision and the resolution of the source signal in order to avoid unnecessary computational effort. This new approach leads to the redefinition and simplification of the model through which waves propagate. The modifications are governed by the spectral characteristics of the source signal. All rays are computed without any omission at a much lower cost in computing time than classical methods. In addition, we will show how to include surface waves such as head waves within the proposed method.

\section{Contributed Papers}

\section{2:40}

3pSP3. Imaging the earth's subsurface via regularized inversion Robert Clapp (Stanford University, 397 Panama Mall, Geophysics Department, Stanford, CA 94305, USA, bob@sep.stanford.edu)

Modern seismic exploration geophysics attempts to construct a model of the earth's subsurface using measurements taken at the earth's surface. The recorded measurements comprise datasets with billions to trillions of samples. Despite the size of the datasets, models are often poorly sampled because of the complexity of the earth's subsurface. Therefore, the resultant model is poorly determined for many components. The massive size of our problems introduce additional limitations. We are limited to adjoint-based inversion methods, we can never afford to iterate to true convergence, and most importantly we make approximations to the physics in our operators. These limitations introduce spurious events when inverting. Significantly improved results can be obtained by incorporating regularization that incorporates a priori knowledge of the physics and geology into the inversion process.

\section{3:00}

3pSP4. Characterization of targets buried in disordered medium Kamal Belkebir (Faculté St Jérôme, 13397 cedex 20 Marseille, France, kamal.belkebir@fresnel.fr), Faheem Ashraf (Faculté St Jérôme, 13397 cedex 20 Marseille, France, faheem.ashraf@fresnel.fr), Marc Saillard (Faculté St Jérôme, 13397 cedex 20 Marseille, France, marc.saillard@1seet.univ-tln.fr), Patrick Chaumet (Faculté St Jérôme, 13397 cedex 20 Marseille, France, patrick.chaumet@fresnel.fr)

We consider in this paper the problem of the determination of the permittivity profile of an unknown buried object from measurements of the electromagnetic scattered field. The target under test is assumed to be buried in one of the two involved media while the sources and the receivers are located in the other medium (limited-aspect data configuration). This illposed and non-linear inverse scattering problem is reformulated as an optimization problem that is solved iteratively. This method consists in building up a sequence of the parameter of interest by minimizing, at each iteration step, a cost functional representing the discrepancy between the data and 
those that would be obtained with the best available estimation of the parameter. In addition, when clutter is present, the decomposition of the time reversal operator method is used to improve the signal-to-clutter ratio, since it allows us to synthesize a wave that focuses on the scatterer. The data associated with this incident field are included in the iterative minimization procedure.

\title{
Invited Paper
}

$3: 20$

3pSP5. Underwater channel characterization using opportunity sources : a time-frequency-phase approach. Cornel Ioana (GIPSA-lab, dep. DIS, 961, rue de la Houille Blanche, 38402 St Martin d'Hères, France, cornel.ioana@ gipsa-lab.inpg.fr), Arnaud Jarrot (Schlumberger Riboud Product Center, 1 rue Becquerel, 92140 Clamart, France, ajarrot@clamart.oilfield.slb.com), Cédric Gervaise (E3I2 - EA3876, 2 rue François Verny, 29806 Brest Cedex, France, cedric.gervaise@ensieta.fr), Andre Quinquis (Scientific Research and Innovation Division, DET/GESMA BP 42, 29240 Brest, France, andre.quinquis@dga.defense.gouv.fr), Jérôme I. Mars (GIPSA-lab, dep. DIS, 961, rue de la Houille Blanche, 38402 St Martin d’Hères, France, jerome.mars@gipsa-lab.inpg.fr)

Analyzing natural signals constitutes the main tool for characterization of physical phenomena. Underwater channel is an example of a natural environment potentially characterized by signals generated by various sources : underwater mammals, human activity noise, etc. In order to efficiently exploit the information from these signals two major problems should be addressed. First, since the signals are unknown or disturbed by unpredictable factors, we are deal with a blind processing context. That is, the lack of a priori hypothesis has to be considered. Generally, the signal has a complex shape characterized by multi-component non-linear time-frequency structures. The proposed solution consists in focusing our processing on non-parametric time-frequency analysis considering also fundamental signal items such as time-frequency energy and local phase analysis. The second problem is related to the complex connection between physical parameters of a phenomenon and parameters of signals characterizing this phenomenon. The proposed approach consists of combining the physical model with the information provided by a parametric representation of the signal. This framework helps to the definition of the concept of underwater passive tomography which provides the characterization of the underwater channel of interest by taking advantage of environmental signals : mammals vocalizations, motion sources, etc.

WEDNESDAY AFTERNOON, 2 JULY 2008

AMPHI BORDEAUX, 2:00 TO 3:20 P.M.

\section{Session 3pUWa}

\section{Underwater Acoustics and ECUA: Fluctuations and Statistics}

\author{
Barry J. Uscinski, Cochair \\ Cambridge University, Centre for Mathematical Sciences, Wilberforce Rd., Cambridge, CB3 OWA, UK \\ Kevin D. Lepage, Cochair \\ Naval Research Laboratory, 4555 Overlook Ave SW, Washington, DC 20375, USA
}

\section{Contributed Papers}

\section{2:00}

3pUWa1. Horizontal structure of acoustic intensity fluctuations in the ocean. Barry J. Uscinski (Cambridge University, Centre for Mathematical Sciences, Wilberforce Rd., CB3 0WA Cambridge, UK, bju1@cam .ac.uk), Jim Nicholson (QinetiQ Ltd., Winfrith Technology Centre, Dorchester, Dorset, DT2 8XJ Dorchester, UK, jrnicholson@qinetiq.com)

In April 1989 an acoustic experiment was performed over the abyssal plain south of Msderia in which transmissions were made, for about an hour, at 482,680 and $740 \mathrm{~Hz}$ from a ship steaming at $5 \mathrm{kts}$ to a receiving array towed by another ship $65 \mathrm{~km}$ away travelling on a parallel course at the same speed. The signals arrived by two paths, an upper path trapped in the surface duct and a lower path via the main sound channel. This paper describes the experiment and analyses the intensity fluctuations in the signal received by the lower path. We investigate the horizontal structure of intensity fluctuations in the ocean when these are mainly due to internal waves. This aspect of such acoustic intensity fluctuations has received little attention until now. The experimental results are compared with theoretical predictions based on the parabolic moment equations for propagation and scattering in randomly irregular media, and on the stnadard Garrett-Munk model for ocean internal waves. The experimental results and theoretical predictions agree quite well but some new questions arise about the correlation of intensity fluctuations as the acoustis transmission frequency is varied.
2:20

3pUWa2. Evolution of the statistics of the unscattered component of low order acoustic modes as a function of range. Tarun K. Chandrayadula (George Mason University, 4400, University Drive, Fairfax, VA 22030, USA, tchandra@gmu.edu), Kathleen E. Wage (George Mason University, 4400, University Drive, Fairfax, VA 22030, USA, kwage@gmu .edu)

Scattering due to internal waves in the ocean causes the acoustic modes to exchange energy as they propagate. At a specific range, the mode signal consists of two components: the unscattered component is the energy that has propagated only in the designated mode and the scattered component contains the contributions from other modes. The unscattered component dominates the signal at short ranges, but decays to zero at longer ranges. If the unscattered component can be isolated from the scattered energy, it could be used in tomographic inversions. Signal processing techniques are needed to detect the unscattered component. A statistical model for the unscattered component that would help design signal processing techniques is currently unavailable. This talk describes a new model for the unscattered component that was developed using coupled mode and parabolic equation simulations. The model characterizes the statistics of the unscattered component using parameters such as frequency and time coherence. The characteristics of the unscattered component are then compared with the scat- 
tered component. The implications of these results for the design of detectors is briefly discussed. [Work supported by ONR Ocean Acoustics Graduate Traineeship Award] moments. The work of PL is supported by The Research of LC was supported by AFOSR and the work of PL is supported by ONR (N00014-06$1-0009)$

\section{3:00}

3pUWa4. Absolute intensities of acoustic shadow zone arrivals. Lora Van Uffelen (MPL, Scripps Institution of Oceanography, University of California, San Diego, La Jolla, CA 92093-0238, USA, lvanuffe@ucsd .edu), Peter Worcester (MPL, Scripps Institution of Oceanography, University of California, San Diego, La Jolla, CA 92093-0238, USA, pworcester@ucsd.edu), Matthew Dzieciuch (MPL, Scripps Institution of Oceanography, University of California, San Diego, La Jolla, CA 920930238, USA,mad@ucsd.edu)

Qualitative observations from bottom-mounted US Navy SOSUS receiving stations in the North Pacific reveal anomalously deep acoustic arrivals at travel times directly corresponding with timefronts expected to have turned much higher in the water column. The vertical structure of these shadow zone arrivals was studied during SPICEX, a long-range propagation experiment conducted from June to November 2004 in the North Pacific, utilizing moored sources 500 and $1000 \mathrm{~km}$ distant from two vertical line array receivers, which together virtually spanned the full ocean depth. Comparison of the measured absolute intensities of shadow zone arrivals with Monte Carlo parabolic equation simulations suggest that the amount of internal wave scattering associated with the standard Garrett-Munk (GM) internal wave spectrum is not adequate to account for the extent of scattering into the acoustic shadow evident in the experimental data, suggesting either that the GM spectrum is not an appropriate representation of the internal wave field or that some other mechanism, such as oceanic spice, may be also be contributing to the scattering.

WEDNESDAY AFTERNOON, 2 JULY 2008

ROOM 341, 2:00 TO 3:40 P.M.

\title{
Session 3pUWb
}

\section{Underwater Acoustics and ECUA: Array Processing}

\author{
David L. Bradley, Cochair \\ Pennsylvania State University, Post Office Box 30, State College, PA 16804-0030, USA \\ Claire Debever, Cochair \\ MPL, Scripps Institution of Oceanography, University of California, San Diego, La Jolla, CA 92093-0238, USA
}

\section{Contributed Papers}

\section{2:00}

3pUWb1. Effect of array element location on coherent inter-array processing. Claire Debever (MPL, Scripps Institution of Oceanography, University of California, San Diego, La Jolla, CA 92093-0238, USA, cdebever@ucsd.edu), William A. Kuperman (MPL, Scripps Institution of Oceanography, University of California, San Diego, La Jolla, CA 92093

\section{8, USA, wkuperman@ucsd.edu)}

In the SWellEx-96 experiment, two 25-elements horizontal arrays, each of length $250 \mathrm{~m}$, were moored $3.5 \mathrm{~km}$ apart [N.O. Booth et al, IEEE, JOE, 25 , no 3, July 2000]. A broadband source (50 to $400 \mathrm{~Hz}$ tones) was towed from $1 \mathrm{~km}$ to $10 \mathrm{~km}$ away from the arrays. The data is processed using the simple conventional plane-wave beamformer for all the cases covering incoherent frequency and incoherent inter-array processing to coherent frequency and coherent inter-array processing as a function of source position relative to the arrays. The effect of noise and array element location mismatch on source localization and coherent processing gain is investigated. An attempt to improve array element localization is made using a selffocusing technique. [Work supported by ONR.]

\section{2:20}

3pUWb2. Longitudinal Correlation Improvement with Model-based Technique. Renhe Zhang (National Laboratory of Acoustics, Institute of Acoustics, Chinese Academy of Sciences, No 21, Beisihuanxilu, 100080 Beijing, China, zrh@mail.ioa.ac.cn), Fenghua Li (National Laboratory of Acoustics, Institute of Acoustics, Chinese Academy of Sciences, No 21, Beisihuanxilu, 100080 Beijing, China, lfh@mail.ioa.ac.cn)

Longitudinal correlation coefficient is one of the most important parameters for signal processing in ocean acoustics. Multi-path interference is an important effect on the decrease of the longitudinal correlation for low frequency sound propagation in shallow water. In this research, the FrequencyPhase-Shift Relationship (FPSR) between the Frequency Response Function (FRF) of sound propagation at two horizontally separated locations based on waveguide invariant is derived. The validity of the FPSR is demonstrated by both numerical simulations and data from two experiments. Experimental data also show that the decrease of the longitudinal correlation coefficient of low frequency signals from explosive source due to multi-path interference can be conquered significantly with the FPSR. [Work supported by the National Natural Science Foundation of China under Grand No 10734100]. 


\section{2:40}

3pUWb3. Performance analysis of GARCH based DOA estimation in SONAR. Hadi Amiri (Engineering Research Institute, Ministry of J-Agriculture, 13445-754 Tehran, Iran, h.amiri@ieee.org), Hamidreza Amindavar (Amirkabir University of Technology, Department of Electrical Engineering, 15914 Tehran, Iran, hamidami@aut.ac.ir), Mahmoud Kamarei (University of Tehran, Department of Electrical and Computer Engineering, 14395-515 Tehran, Iran, kamarei@ut.ac.ir)

In this paper we propose a new source localization method using additive noise modeling based on Generalized Autoregressive Conditional Heteroscedasticity (GARCH) time-series. In an actual application such as underwater acoustics, the measurement of additive noise in a natural environment shows that noise can sometimes be significantly non-Gaussian and nonstationary, and therefore, signal processing algorithms that are optimized for Gaussian noise, may degrade significantly in this environment. GARCH models are feasible for heavy tailed PDFs and time varying variances of stochastic process and also has flexible forms. We use a more realistic $\operatorname{GARCH}(1,1)$ based noise model in the Maximum Likelihood Approach for the estimation of Direction-Of-Arrivals (DOAs) of impinging sources. In the performance analysis of the method, we examine the suitability of the proposed method in a passive sonar using simulation methods with cramer-rao bound (CRB) and perturbaion appraoch such as gain, phase and sensors positions errors.

\section{3:00}

3pUWb4. Direct wave suppression using wavelet transform for bistatic sonar. Bo Lei (Institute of Acoustic Engineering, Northwestern Polytechnical University, 710072 Xi'an, China, lei.bo.thunder@gmail .com), Yuanliang Ma (Institute of Acoustic Engineering, Northwestern Polytechnical University, 710072 Xi'an, China, ylma@nwpu .edu.cn), Kunde Yang (Institute of Acoustic Engineering, Northwestern Polytechnical University, 710072 Xi’an, China, ykdzym@nwpu .edu.cn), Chao Sun (Institute of Acoustic Engineering, Northwestern Polytechnical University, 710072 Xi’an, China, csun@nwpu.edu.cn)

The bistatic sonar is often influenced by the direct wave, especially when the target approaches the baseline of the system. The direct wave is above 50dB higher than the scattering signal level. The paper analyzes the effect of the direct wave, and proposes a new method based on wavelet transform for direct wave suppression. The strong direct wave is well eliminated while maintaining the weak scattering signal almost unchanged. The basic idea is that the direct wave is firstly removed form the received signal after wavelet transform. Then the signal is reconstructed in time domain, finally the cross-correlation is achieved to shown the doppler frequency shift of the weak scattering signal. Computer simulation is given. The crosscorrelations of the received signal for both before and after the direct wave suppression are compared. It shows that the influence of the direct wave is correctly removed, the time delay and Doppler frequency shift are shown in the figure and the target is detectable.

\section{$3: 20$}

3pUWb5. Adaptive beamforming applied to underwater acoustic measurements. Denis Orlov (Institute of Applied Physics of the Russian Academy of Sciences, 46 Ulyanov Street, 603950 Nizhny Novgorod, Russian Federation, denis@hydro.appl.sci-nnov.ru), Iosif Fiks (Institute of Applied Physics of the Russian Academy of Sciences, 46 Ulyanov Street, 603950 Nizhny Novgorod, Russian Federation, fiks@hydro.appl .sci-nnov.ru), Galina Fiks (Institute of Applied Physics of the Russian Academy of Sciences, 46 Ulyanov Street, 603950 Nizhny Novgorod, Russian Federation, galya@hydro.appl.sci-nnov.ru), Pavel Korotin (Institute of Applied Physics of the Russian Academy of Sciences, 46 Ulyanov Street, 603950 Nizhny Novgorod, Russian Federation, monitor@hydro.appl .sci-nnov.ru), Victor Turchin (Institute of Applied Physics of the Russian Academy of Sciences, 46 Ulyanov Street, 603950 Nizhny Novgorod, Russian Federation, tvi@hydro.appl.sci-nnov.ru)

In many applications, such as measuring the underwater noise level of moving ships, underwater acoustic measurements face serious difficulties related to several factors, including low signal-to-noise ratio, effects of surface and bottom on signal, as well as complicated spatial structure of the sea interference. The increase of the measurement interference resistance, compared to the case of a single receiver (hydrophone), can be provided by the use of spatially distributed receiving systems (antenna arrays), which are able to suppress the interference due to their spatial selectivity. The present work is aimed at developing adaptive methods for underwater acoustic measurements with the use of vertical antenna arrays. The method must provide the maximum reduction of external interference keeping the given measurement accuracy, i.e., the result must coincide with the output of a single receiver in the absence of interference. From the point of view of synthesis of array systems, the originality of the presented approach is mainly in conjunction of measurement functionality of the antenna array and the maximum interference suppression. The results of numerical simulation and experimental testing under sea conditions show that the proposed adaptive methods provide high precision of measurements under strong and/or complex interference conditions. 


\title{
Session 3pUWc
}

\section{Underwater Acoustics and ECUA: Objects Scattering and Detection}

\author{
Sven M. Ivansson, Cochair \\ Swedish Defence Research Agency, FOI Kista, Stockholm, SE-16490, Sweden \\ Ahmad Abawi, Cochair \\ HLS Research, Inc., 3366 N. Torrey Pines Ct., Ste. 310, La Jolla, CA 92037, USA
}

\section{Contributed Papers}

\begin{abstract}
2:00
3pUWc1. Numerical design of Alberich anechoic coatings with superellipsoidal cavities of mixed sizes. Sven M. Ivansson (Swedish Defence Research Agency, FOI Kista, SE-16490 Stockholm, Sweden, sveni @ foi.se)

Thin rubber coatings with cavities in a doubly periodic lattice are able to reduce reflections of underwater sound by redistributing normally incident energy such that absorption in the surrounding rubber is enhanced. For spherical scatterers, the anechoic effect can be studied numerically by the layer multiple-scattering method. In comparison to more flexible but also more computer intensitive methods, such as FEM modeling, there are two important advantages. An improved physical understanding of the anechoic effect can be achieved by simplified semianalytical analysis, and the high computational speed allows modern global optimization techniques to be applied for coating design. In this paper, the flexibility of the layer multiscattering method is improved by combination with an efficient algorithm for numerical computation of transition matrices for superellipsoidal scatterers. Extensions to mixtures of nonspherical scatterers of different types are also considered, in order to enhance the broad-band performance. Symmetry properties are used to reduce the size of the pertinent equation systems. Examples of numerical coating design for underwater acoustic applications are presented, using differential evolution algorithms for the optimization.
\end{abstract}

\section{2:20}

3pUWc2. Scattering from rotationally-symmetric objects using only free space Green's functions. Ahmad Abawi (HLS Research, Inc., 3366 N. Torrey Pines Ct., Ste. 310, La Jolla, CA 92037, USA, Abawi@HLSResearch.com), Michael B. Porter (HLS Research, Inc., 3366 N. Torrey Pines Ct., Ste. 310, La Jolla, CA 92037, USA, michael.porter @ hlsresearch.com)

To compute scattering from an object, one has to solve the wave equation and impose the appropriate boundary conditions on the surface of the object. For objects for which the wave equation can be separated, like cylinders, spheres and spheroids, this problem can be solved analytically. For more general objects, methods like the finite element or boundary element techniques can be employed. The use of free space Green's functions offers another method for computation of scattering from a general-shaped object. This method, which is known as the method of field superposition or the virtual source technique, can be used to impose the appropriate boundary conditions on the surface of the object by using free space Green's function with complex amplitudes. These amplitudes are determined from a matrix equation that results when boundary conditions are imposed. In this paper we apply this technique to compute scattering from rotationally symmetric objects. These objects can be homogeneous solids or shells, filled with a homogeneous fluid. The versatility and robustness of the method is demonstrated by applying it to various objects in free space and in a waveguide.

\section{2:40}

3pUWc3. Modeling of underwater sonar barriers. Andrzej Elminowicz (R\&D Marine Technology Centre, Ul. Dickmana 62, 81-109 Gdynia, Poland, andrzeje@ctm.gdynia.pl), Leonard Zajaczkowski (R\&D Marine Technology Centre, Ul. Dickmana 62, 81-109 Gdynia, Poland, leonard@ctm.gdynia.pl)

Commonly used Diver Detection Sonar (DDS) are characterized by detection and tracking as well as intruder warning in limited range $300 \mathrm{~m} \div$ $600 \mathrm{~m}$. A new approach to underwater protection is focused on multimonostatic, bistatic and multistatic active sonar barriers (ASB). This paper describes key features of a multi-monostatic (MM), bistatic (BS) and multistatic (MS) operation in the littoral beginning from system configuration, its performance and constraints. A new type of acoustic devices in a form of a transmitting/receiving module and its application in active sonar barriers (ASB) has also been presented. Coverages and ranges of barriers for various technical parameters, environmental conditions and wave propagation as well as modules location (including transducers' depth, azimuth and inclination) have been studied. The application of active sonar barriers (ASB), especially in protection systems, has been presented as barriers protecting docks, harbour basins, ships at piers or harbour entries. The new type of a monostatic/bistatic acoustic barrier in technology demonstrator form, has been designed and constructed. In order to display detection and localization of the small underwater objects as well as their tracking and classification the performance tests of the barrier will be conducted. The results of the tests will be attached to the paper.

\section{3:00}

3pUWc4. Wideband sonar system for autonomous surveys using REMUS. Chris Capus (Ocean Systems Laboratory, School of Eng. \& Phys. Sciences, Heriot-Watt University, EH14 4AS Edinburgh, UK, C.Capus@hw.ac.uk), Yan Pailhas (Ocean Systems Laboratory, School of Eng. \& Phys. Sciences, Heriot-Watt University, EH14 4AS Edinburgh, UK, Y.Pailhas@hw.ac.uk), Keith E. Brown (Ocean Systems Laboratory, School of Eng. \& Phys. Sciences, Heriot-Watt University, EH14 4AS Edinburgh, UK, K.E.Brown@hw.ac.uk)

Many new roles are being proposed for Autonomous Underwater Vehicles (AUVs) to carry out hazardous tasks in harsh or remote locations and to free up valuable resources required for manned missions. These roles include marine environmental survey, target detection and classification and tracking of underwater pipes and cables. Suitable sensing and processing packages must be provided and building on recent wideband sonar research, the Ocean Systems Laboratory (OSL) is putting together a wideband system for deployment on board a REMUS AUV. The prototype sensor package comprises paired projectors and receivers mounted in a side-looking arrangement to provide complementary information to the standard REMUS sidescan modules. The sensor bandwidths cover a range from $30-130 \mathrm{kHz}$ similar to those used by the bottlenose dolphin. Data is gathered autonomously with a dedicated AMD Geode based PC104+ PC controlling a 4-channel $800 \mathrm{kHz}$ simultaneous sampling data acquisition module. Acquisition is triggered to coincide with mission requirements from a separate mission control PC on board the vehicle. 


\section{3:20}

3pUWc5. A multistatic synthetic aperture sonar to detect a cylinder lying on a rough interface: experimental results. Caroline Herve (Laboratory for Mechanics and Acoustics CNRS, 31 chemin Joseph Aiguier, 13009 Marseille, France, herve@1ma.cnrs-mrs.fr), Jean-Pierre Sessarego (Laboratory for Mechanics and Acoustics CNRS, 31 chemin Joseph Aiguier, 13009 Marseille, France, sessarego@lma.cnrs-mrs.fr), Régine Guillermin (Laboratory for Mechanics and Acoustics CNRS, 31 chemin Joseph Aiguier, 13009 Marseille, France, guillermin@1ma.cnrs-mrs .fr), Françoise Schmitt (Groupe d'Electromagnétisme Appliqué, Pôle Scientifique et Technologique de l'Université Paris X Nanterre Site de Ville d'Avray, 50, rue de Sèvres, 92410 Paris Ville-d'Avray, France, fschmitt@u-paris10.fr), Franck Daout (Groupe d'Electromagnétisme Appliqué, Pôle Scientifique et Technologique de l'Université Paris X Nanterre Site de Ville d'Avray, 50, rue de Sèvres, 92410 Paris Ville-d'Avray, France, fdaout@u-paris10.fr)
Monostatic Synthetic Aperture Sonar (SAS) are high resolution systems for target imaging which are now of common use in the underwater acoustics domain. The objective of this work was to analyse what kind of information should be obtained from a multistatic SAS system. This idea has been applied in radar but very few works exist in the underwater acoustics domain. The applications could be detection and identification of buried mines or mines lying on the seabed and divers detection for harbour protection. These applications deal with the problem of target detection and identification near a rough surface. To show what can be obtained with a multistatic SAS processing, we have performed an experiment with a circular cylinder of $1 \mathrm{~cm}$ diameter lying on a rough interface made of sand grains of $1 \mathrm{~mm}$ diameter. Measurements were performed in a tank with both a multistatic and a monostatic SAS systems. The signal used to insonified the target area was a short impulse with a $2 \mathrm{MHz}$ central frequency which corresponds to a ka about 40 . Images of the cylinder in presence of clutter have been reconstructed with the matched filtering algorithm from monostatic and multistatic acquisitions and have been compared. 


\title{
Awards Ceremony
}

\author{
Gilles A. Daigle \\ President, Acoustical Society of America \\ Luigi Maffei \\ President, European Acoustics Association \\ Jean Kergomard \\ President, Société Française d'Acoustique
}

\section{Acoustical Society of America}

\section{Presentation of ASA Fellowship Certificates}

\author{
Michael A. Akeroyd \\ Susanna B. Blackwell \\ Dick B. Botteldooren \\ Dani M. Byrd \\ Brian R. Glasberg \\ Larry E. Humes \\ Vera A. Khokhlova
}

Christophe D. Micheyl

Anthony P. Nash

John C. Osler

Subramaniam D. Rajan

Richard M. Stern

Brad H. Story

Lily M. Wang

2007 Research Grant in Speech Science of the American Speech-Language-Hearing Foundation to Mary K. Fagan

\author{
Medwin Prize in Acoustical Oceanography to Mark V. Trevorrow \\ Distinguished Service Citation to Charles E. Schmid \\ R. Bruce Lindsay Award to Tyrone M. Porter \\ Helmholtz-Rayleigh Interdisciplinary Silver Medal to James V. Candy \\ Gold Medal to Patricia K. Kuhl

\section{European Acoustics Association}

Award for Lifetime Achievements in Acoustics to Gunnar Rasmussen

Award for Contributions to the Promotion of Acoustics in Europe to the Federation of Acoustical Societies of Europe

(FASE) 1971-1996

Award for Outstanding Recent Scientific Results Published in Acta Acustica united with Acustica to Catherine Guastavino, Brian F. G. Katz, Jean-Dominique Polack, Daniel J. Levitin, and Danièle Dubois

\section{Société Française d'Acoustique}

The French Medal to Vitalyi Gusev

The Foreign Medal to Walter Lauriks 


\title{
Session 3eID
}

\section{Plenary Session}

\author{
Introduced by Dick Botteldooren, Ghent University, Belgium
}

3eID1. Binaural Hearing and Systems for SOund Reproduction. Philip A. Nelson (ISVR, University of Southampton, Highfield, SO171BJ Southampton, UK, P.A.Nelson@aoton.ac.uk), M. Park (ISVR, University of Sothamton, Highfield, SO171BJ Southamption, UK, unknown@unknown.com), Takashi Takeuchi (OPSODIS Limited, c/o ISVR, University of Southamption, Highfield, SO17 1BJ Southampton, UK, ff1@isvr.soton.ac.uk)

Recent developments in models of binaural hearing can be usefully adapted and extedned to provide design tools for engineers engaged in the design of systems for sound reproduction. The particular focus of the work described is upon the development of models that give good statistical predictions of human sound localisation, based upon knowledge of the fluctuating acoustic pressures at the ears. Such models can be applied successfully to the prediction of stereophonic image localisation and reveal a number of important features of localisation relevant to audio system design. Developments will also be described in loudspeaker based systems for binaural reproduction that are finding their way into practical use. Binaural hearing models can be used to provide a preliminary evaluation of the performance of alternative designs. Finally, a brief review will be presented of multi-channel loudspeaker-based systems aimed at "full field" sound reproduction. Again, models of localisation provide some useful guidance for the designers of such systems.

Introduced by William M. Hartmann, Michigan State University, USA

3eID2. Optoacoustics: Can ultrasound become the preferred modality for molecular imaging? Matthew O'Donnell (University of Washington, 371 Loew Hall, Box 352180, Seattle, WA 98195-2180, USA, odonnel@engr.washington.edu)

Optoacoustics can be used to image the distribution of optical absorption in tissue, combining the specificity and sensitivity of optical imaging with the high resolution and penetration of ultrasound imaging. It represents one of the most promising techniques for molecular imaging because the optical absorption of bioconjugated nanoparticles can greatly exceed that of tissue over a range of wavelengths in which light can penetrate multiple centimeters into the body. We have explored several types of nanoparticles conjugated to a range of antibodies targeted to several important biological systems. Here we discuss two potential applications of bioconjugated gold nanorods, one for cancer cell targeting and the other to identify inflamed endothelial cells signaling the early stages of atherosclerosis. Optoacoustic images of cell cultures and animal models demonstrate the sensitivity and specificity of these nanosystems for molecular imaging. To translate these experimental findings into a clinically acceptable molecular imaging modality, we have also explored integrated optical systems able to deliver the optical pulse for optoacoustic excitation and detect the resultant ultrasonic waves using an all-optical transducer. The basic operating principles of this device, and the prospects for ultrasound-based molecular imaging using it, will be discussed. 


\title{
ACOUSTICAL SOCIETY OF AMERICA GENERAL ASSEMBLY
}

\author{
Acoustical Oceanography Prize Lecture
}

\author{
James H. Miller, Chair \\ Univ. of Rhode Island, Dept. of Ocean Engineering, Narragansett, RI 02882
}

Chair's Introduction-5:40

\begin{abstract}
$5: 45$
The use of moored inverted echosounders for monitoring near-surface processes. Mark Trevorrow (Defence R\&D Canada Atlantic, 9 Grove St., PO Box 1012, Dartmouth, NS B2Y 3Z7, Canada, mark.trevorrow@drdc-rddc.gc.ca)

The near-surface ocean is a difficult place to conduct measurements of physical and biological processes. High-frequency inverted echo-sounders (IES), moored a short distance below the surface, provide a simple means for sustained, high-resolution monitoring. In particular, long duration deployments are useful in capturing transient events, such as storms or solitary wave trains, and placing their occurrence within the context of longer term trends. Lessons learned from use of these moored IES systems can be applied to modern ocean observatories. A number of long duration deployments of a self-contained 200kHz IES were made in the late 1990's. Three specific examples will be discussed. Firstly, an investigation of calibrated volume scattering from near-surface bubbles in the NE Pacific Ocean showed the frequent occurrence of vertical, plume-like structures drawn downwards up to $25 \mathrm{~m}$. Within the plumes, the backscatter cross-section exhibited an exponential decay with depth, with e-folding scale in the range 0.5 to $2.5 \mathrm{~m}$. Secondly, imaging of near-surface internal solitary waves was performed from a mooring near the Oregon coast, supplementing measurements made with nearby temperature sensors. These measurements showed some suggestion of scattering by turbulence. Thirdly, through the use of both backscatter intensity and echo statistics, a moored IES demonstrated quantitative monitoring of migratory zooplankton and fish populations.
\end{abstract}

WEDNESDAY EVENING, 2 JULY 2008

AMPHI BORDEAUX, 5:40 TO 7:20 P.M.

\section{Celebration of the $60^{\text {th }}$ Anniversary of the French Acoustical Society}

On June 25, 1948, the so-called "Groupement des Acousticiens de Langue Française" (GALF) was created, with its first President, Yves Rocard, and was transformed into the "Société Française d'Acoustique" (SFA) in 1986. SFA now has 830 members and is composed of 8 technical groups and 3 regional sections. It is one of the founding members of the European Acoustics Association (EAA).

The $60^{\text {th }}$ anniversary will be focused on the presentation of the "White Book" of Acoustics in France, and the publishing of a DVD including the main part of the French journals in Acoustics since 1934.

\section{Agenda}

Opening by the current President of SFA and a former President (René-Guy Busnel)

Acoustical French journals since 1934: Presentation of the DVD

The White Book of Acoustics in France in 2008

White Book: An example of the position of acoustics in an activity sector: The automotive industry

60 years of acoustics in France: An overview 